\title{
DEVELOPMENT OF A BIOTECHNOLOGICALLY ENHANCED STERILE INSECT TECHNIQUE TO FIGHT COLEOPTERAN PESTS
}

\author{
Dissertation \\ for the award of the degree \\ "Doctor rerum naturalium" (Dr.rer.nat.) \\ of the Georg-August-Universität Göttingen
}

within the doctoral program 'Biology'

of the Georg-August University School of Science (GAUSS)

Submitted by

Musa Dan'azumi Isah

From Bauchi, Nigeria

Göttingen, 2021

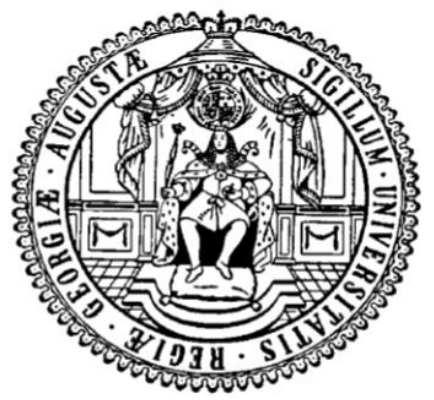




\section{Thesis Committee}

Prof. Dr. Ernst A. Wimmer

Entwicklungsbiologie - Johann-Friedrich-Blumenbach-Institut für Zoologie und Anthropologie Georg-August-Universität Göttingen

Prof. Dr. Gregor Bucher

Evolutionäre Entwicklungsgenetik - Johann-Friedrich-Blumenbach-Institut für Zoologie und Anthropologie, Georg-August-Universität Göttingen

Members of the Examination Board

First Reviewer: Prof. Dr. Ernst A. Wimmer

Entwicklungsbiologie - Johann-Friedrich-Blumenbach-Institut für Zoologie und Anthropologie Georg-August-Universität Göttingen

Second Reviewer: Prof. Dr. Gregor Bucher

Evolutionäre Entwicklungsgenetik - Johann-Friedrich-Blumenbach-Institut für Zoologie und Anthropologie -Georg-August-Universität Göttingen

Prof. Dr. Ralf Heinrich

Zelluläre Neurobiologie, Schwann-Schleiden Research Centre, Johann-FriedrichBlumenbach-Institut für Zoologie und Anthropologie -Georg-August-Universität Göttingen

Prof. Dr. Micheal Rostàs

Georg-August-Universität Göttingen, Department für Nutzpflanzenwissenschaften

Abteilung Agrarentomologie, Grisebachstrasse 6

D-37077 Göttingen

Prof. Dr. Christoph Bleidorn

Department of Animal Evolution and Biodiversity, Johann-Friedrich-Blumenbach-Institut für Zoologie und Anthropologie -Georg-August-Universität Göttingen, Untere Karspuele 3, 37073, Göttingen, Germany

Prof. Dr. Daniel Jackson

Evolution der Metazoen, Geoscience Centre - Georg-August-Universität Göttingen, Goldschmidtstr. 3, 37077 Göttingen, Germany

Date of oral examination: 01.03.2021 


\section{Declaration:}

This doctoral thesis titled "DEVELOPMENT OF A BIOTECHNOLOGICALLY ENHANCED STERILE INSECT TECHNIQUE TO FIGHT COLEOPTERAN PESTS"

is a product of my independent doctoral research work, conducted at the Department of Developmental Biology, Georg-August-University Göttingen. This research work is novel and has not been submitted elsewhere for the award of any degree. Works of other people cited herein have been acknowledged by reference. 


\section{Dedication}

To my beloved wife Angela and lovely daughter Layum Christabel

and

my parents and siblings 


\section{Acknowledgement:}

I want to specially thank my supervisor and my academic model/mentor Prof. Dr. Ernst A. Wimmer for offering me a doctoral position in his lab, and then for giving me the freedom to express myself in a scientific manner and also the resources necessary to make this study a success. I also appreciate Prof. Dr. Gregor Bucher for being my co-supervisor of this work and for his advice, suggestion and fruitful discussion which helped to make this research work possible. Special thanks are due to those who always support me with open heart and smile i.e. Bettina Hucke, Birgit Rossi and Merle Eggers. My thanks and appreciations particularly go to Stefan Dippel for his patience and gracious dispensation for bearing with my inadequacies during my embryonic period in the laboratory and seeing me through basic molecular biology technique. I will also like to thank my colleague and friends in Lab 1: Hassan Mohammed Mutasim Ahmed, Kolja N. Eckermann, Ingrid Curril, Bibi Atika for our long years of working together, and also to my express my indebtedness for their assistance in the application of useful molecular biology tricks. I also want to seize this opportunity to thank my colleagues and friends in the general department, Elisa Buchberger, Micael Reis, Amel Chtioui, Felix Quade, Dominik Muehlen, Marita Büscher and Max Farnworth for providing a nice and healthy laboratory atmosphere for me to function efficiently. My thanks are also due to Evangelish Studientenwerk Villights and University of Calabar, Nigeria for financial support during the period of this studies. To also, our technical staff at the department of developmental biology, Helma Gries, Angelika Löffers, Elke Küster and Katrin Kanbach for their readiness and willingness to help I said thanks. My thanks and gratitude are also due to Daval, Tarek and Beate Preitz for their help regarding computer problems and especially Beate Preitz for introducing me to various microscopy maneuver and techniques. I would also love to send sincere thanks and gratitude to my parents (Mr. and Mrs. Dan'azumi Isah) and siblings (Ishaku, Liatu and Hajara) for all the unconditional support throughout the years. Finally, I would like to express my thanks and indebtedness and gratitude to my beloved wife Angela and my lovely daughter Layum Christabel for being there for me and for unconditional support during the whole period of this doctoral study. 
Table of contents:

Title page.

Thesis committee page.

Declaration. ..i

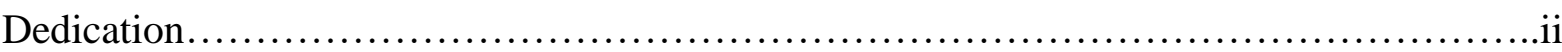

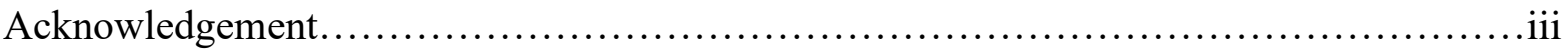

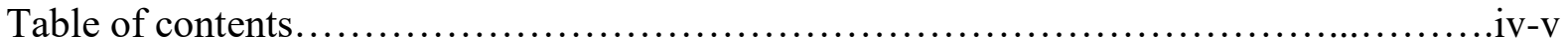

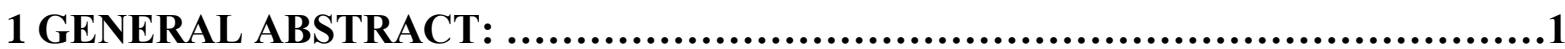

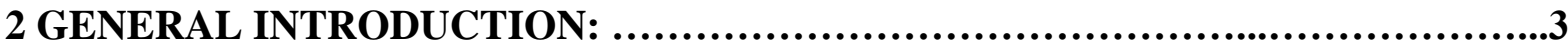

2.1 Red Flour Beetle (RFB) Tribolium castaneum (Tenebrionidae) as a coleopteran model species: ..........................................................3

2.2 Molecular approaches to analyze gene function in RFB.....................4

2.2.1 Transposon mediated insect transgenesis.............................4

2.2.2 RNAi mediated gene silencing mechanism: ................................5

2.2.3 CRISPR-Cas9 genome editing...........................................5

2.2.4 Site specific recombination: ........................................6

2.3 Classical Insect Pest Control Measures.......................................

2.3.1 Physical/Cultural methods: ..........................................7

2.3.2 Biological methods: .................................................7

2.3.3 Chemical methods: .................................................

2.3.4 Integrated Pest Management (IPM): ................................7

2.4 The Sterile Insect Technique (SIT) and its potential: ........................8

2. 4.1 Suitability of sterile insect technique for the control and management of insect pests: .........................................................10

2.4.2 Transgenic sterile insect technique to generate conditional male sterile marked insect pests: ................................................10

2.4.3 Transgenic sexing for male-only release: ............................11

2.4.4 Transgenesis as a suitable alternative in achieving radiation sterilization replacement prior to release: ................................11

2.4.5 Monitoring of released transgenic insects: ............................12

2.4.6 Transgenic insect stability: .........................................13

2.5 Objectives of research: ...................................................14 
3 RESULTS: .16

3.1 Transgenic sperm marking to enhance monitoring of coleopteran insect pests: .17

3.2 Identification and characterization of early embryonic gene(s) for the development of transgenic embryonic lethality systems in Tribolium castaneum: .58

3.3 Novel genetic engineering approach for the management of coleopteran insect pests using CRISPR-Cas9: .97

3.4 Genetic improvement and use of CRISPR/Cas9 to generate an early embryonic driver line in Tribolium castaneum: .119

4 General discussion: .145

4.1 Transgenic sperm marking in Tribolium castaneum: .145

4.2 Identification and characterization of potential cellularization gene(s) in

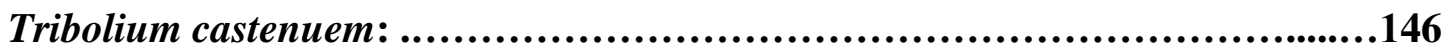

4.3 Female Specific Embryonic Lethality systems (FSELs):...................147

4.4 Genetic Improvement and Use of CRISPR/Cas9 in Tribolium castaneum for genome editing: .148

4.5 Novel genetic engineering approach for the management of coleopteran (Tribolium castaneum) insect pests using CRISPR-Cas9 system for reproductive sterility: 149

5 Outlook: .151

6. References: .152

6 Curriculum Vitae: .170 


\section{GENERAL ABSTRACT}

Tribolium castaneum commonly referred to as the Red Flour Beetle (RFB) belongs to the class Insecta and order Coleoptera, and it's the best model organism for coleopterans. About 380,000 coleopteran species, constituting approximately $25 \%$ of all the animal species described in the world have been identified, thus making them the most taxonomically described species in the animal kingdom, and they are found occupying different ecosystems due to their extraordinary diversities. Approximately $75 \%$ of the beetle described are polyphagous as larvae and adult, and are found to live on plants, wood products and stored products and are of economic importance for agriculture, as well as forestry and other household products. By such activities they cause several and significant damage both in direct and indirect losses. The current predominant control measure employed for controlling coleopteran pests is the injurious application of chemicals which results in the development of resistance over time, and also have negative effect to the environment and human health. Hence, there is a need for more efficient and environmentally friendly control measures that is devoid of the drawbacks identified, hence the development and usage of biotechnological approaches.

Transgenic approaches use recombinant DNA via genetic engineering to mimic classical genetic approaches, which is also less expensive, and addresses the drawbacks of the classical genetic approach. However, while these practices have been employed in various insect pests mostly in the order of dipteran, none has been employed to any coleopteran insect pest so far. Biotechnological approaches could improve techniques such as sexing for male alone releases, sperm marking, and female/embryonic specific lethality strategies, where classical genetic approaches use for example radiation for causing sterility, fluorescent dyes for monitoring and phenotypic strategies such as pupal sizes or colour for sexing which have several disadvantages.

Tribolium castaneum as a model insect pest has a comprehensive genome database, which can be utilized for both evolutionary and population genetics approaches. The organism is also the most utilized insect for development biology studies aside from Drosophila melanogaster due to the ease of handling the insect in the laboratory, short generation time, high fecundity, etc. Here I provide the first attempt and proof of principle of transgenic approaches for sterility, sperm marking for monitoring, and sexing using recombinant DNA. Firstly, for monitoring, I used the Promoter/Enhancer (P/E) region of the Tribolium spermatogenesis specific $\beta 2$ tubulin gene to drive DsRed and/or EGFP fluorescent protein in the sperm of male individuals by first integrating the constructs by piggyBac germline transformation to generate transgenic 
lines. I observed the marked sperm in the testis of the male, and in the spermatheca of the female when a wildtype female is mated with a transgenically sperm-marked male individual. This can solve the drawback that is observed in the bodily application of fluorescent dyes on the released individuals, which will result in either false negative or false positive in the classical SIT in capture and recapture experiments. Also, the generated sperm marked individuals provided us with a tool to answer some reproductive biology question on sperm storage and usage by twice mated females. The result showed that the female store the sperm for a long period, and use the sperm of the second inseminated males first, with the first male inseminated sperms used subsequently.

Moreover, we discovered a new early embryonically expressed gene ( $T c 007675)$ which was hitherto not reported and used it for functional studies. I used the amplified $1.4 \mathrm{~Kb}$ or $2.1 \mathrm{~Kb}$ of the Promoter/Enhancer (P/E) upstream region of the gene to drive the artificial heterologous transactivator tTA in order to generate several driver strains by piggyBac germline transformation. By Whole Mount In Situ Hybridization (WMISH), two driver lines expressing tTA at cellularization stage were identified. In addition, using CRISPR-Cas9 genome editing, I effectively generated a driver line targeting the Tc007675 gene locus by Homology Directed Repair (HDR) strategy to generate a bi-cistronic locus. The effectiveness of the driver line was verified using WMISH, where we detected a transient expression of tTA under the control of the $T c 007675$ gene.

Furthermore, using the spermatogenesis specific B2t enhancer/promoter to drive tTA, we generated a driver line that expresses tTA in testis of one of the lines generated. With this line, we intend to express Cas9 and respective guide RNAs in the testis as a new approach to cause reproductive sterility. At the same time, we generated a potential Cas 9 expressing effector line which we crossed with our driver line but for now we were not able to drive Cas9 in the testis. This idea will be used to mimic the use of ionizing radiation used in classical approach, where it could take care of the drawback arising from fitness loss due to the mutation effect on somatic cells of the treated organism. The CRISPR-Cas9 mediated reproductive sterility system will provide a novel and efficient way, in which males will have no fitness or competitiveness disadvantages to wildtype when released. 


\section{GENERAL INTRODUCTION:}

\subsection{Red Flour Beetle (RFB) Tribolium castaneum (Tenebrionidae): Coleopteran model insect pest:}

The class Insecta is the largest group of organisms ${ }^{1}$ in the animal kingdom, and their successes can be attributed to their ability to survive in different niches such as water bodies ${ }^{2}$, tropical rain forest ${ }^{3}$, several land areas such as mountains, caves, hot spring, polar zones and their staggering diversity ${ }^{4}$. Coleopterans insect also known as beetles belong to the class Insecta, and comprises about 380,000 described species ${ }^{5,6}$ of the class, thereby making them about $\sim 40 \%$ of all described species ${ }^{7}$, and known to occupy different ecological niches ${ }^{8}$.

Tribolium casteneum otherwise called The Red Flour Beetle, ${ }^{9}$ belongs to the order Coleoptera., and it is the coleopteran model organism ${ }^{10}$, and its second only to Drosophila melanogaster (Order: Diptera), for various genomic studies due to: 1) Easy of handling 2) High fecundity and 3) Easy to maintain in a laboratory ${ }^{17}$. The organism is helping in answering several developmental and evolutionary biology questions ${ }^{11}$ which hitherto cannot be investigated.

Female $T$. casteneum have the capacity to lay about $200-500$ eggs within a total lifespan of about 3 years ${ }^{11}$. T. casteneum is found to develop favorably at $27^{\circ} \mathrm{C}^{11}$, with the first instar larvae looking slender and wormlike in appearance ${ }^{12}$. The larvae are covered with fine hairs, and also possess a spine like structure called urogomphi ${ }^{11}$ found at the last abdominal segment, and sexing of individuals are mostly done at the pupal stage ${ }^{13}$ by the possession of lobe like structure notices at the last abdominal segment in the female pupae which is not seen in male pupae.

Tribolium casteneum are primarily secondary pest that infests stored nuts and cereals, with however fungal infested wood suggested to be its original habitat ${ }^{14}$. The insect is found to occupy all ecological niches due to it tolerance to both warm and cold temperatures ${ }^{15}$, and also its ability to infest animal food stores ${ }^{16}$ apart from nuts and cereals, which is due to the availability of its gustatory receptors genes. 


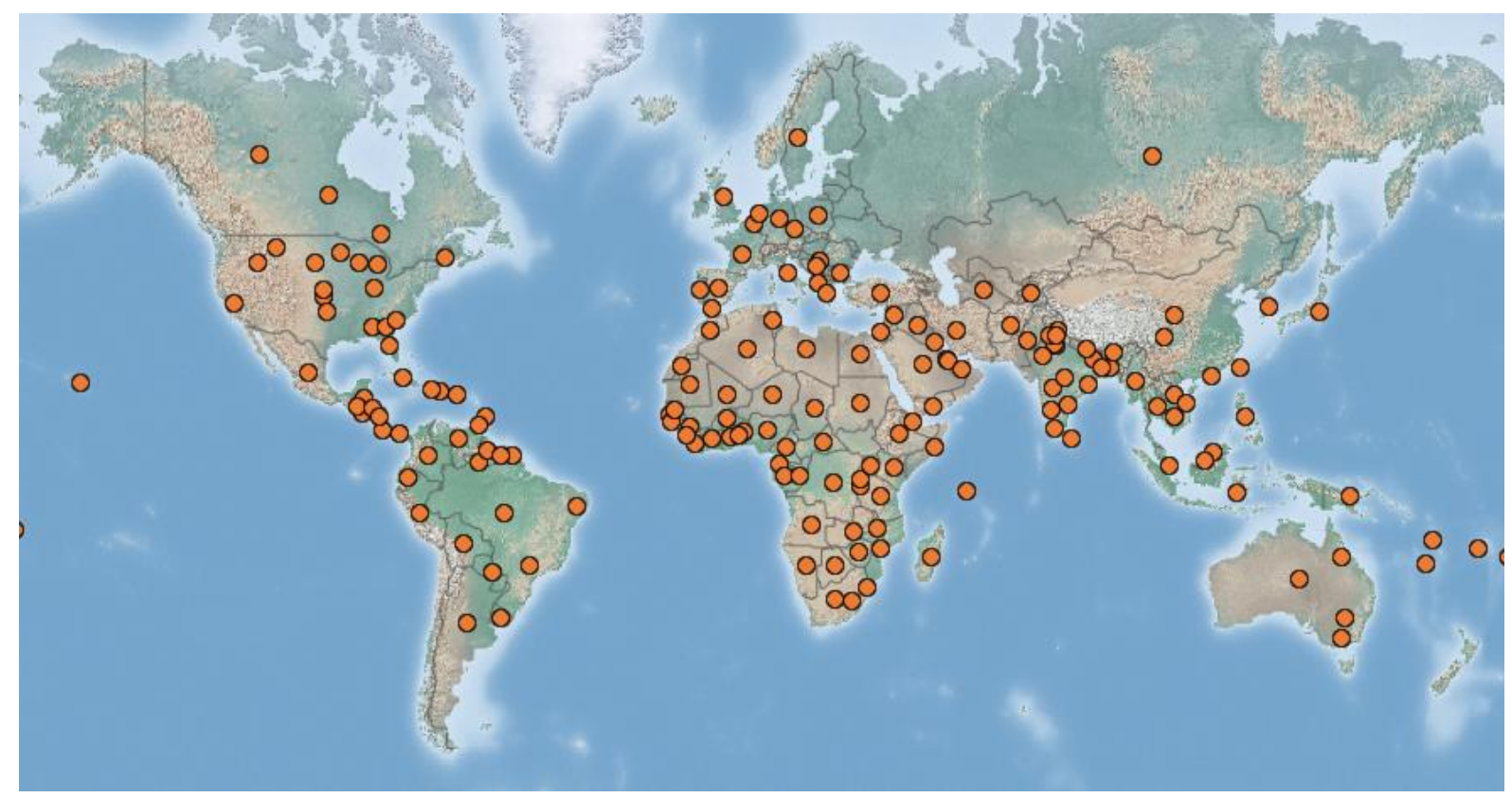

https www.cabi.org_isc_distribution_map

Tribolium casteneum being a popular model for genetic is also use for population differentiation ${ }^{18}$, and host-parasite interaction ${ }^{19}$ investigation. It is the first coleopteran species, with its full genome sequenced ${ }^{16}$ that is compressively annotated, also it is useful as genetic tool for gene locus studies availability, order and distances between genetic markers identification 20 and for transformation studies involving transposons 10,11,18 elucidated. Also, the organism has been used for several transgenic experiments ${ }^{21}$, insertional mutagenesis ${ }^{22}$, Crispr-Cas 9 experiments ${ }^{23}$ among others.

\subsection{Molecular approaches to analyses gene function in RFB}

\subsubsection{Transposon mediated insect transgenesis:}

Insect genetic transformation using $P$ element ${ }^{24}$ was first used for Drosophila melanogaster almost 40 years ago, but the element was unfortunately not found to work in other insect species except in Drosophila spp ${ }^{25}$ which could be due to factors associated with host-specificity 2627. However, the genetic transformation of other insect pest species is now possible due to the discovery of other transposon ${ }^{28,29}$ such as Hermes ${ }^{30,31}$ Mos $^{32}{ }^{32}$ Minos $^{33}$, and piggyBac ${ }^{34}$ etc. These new transposons have being used successfully in mediating the transformation of other insect orders 29,35 36,37. 


\subsubsection{RNAi mediated gene silencing mechanism:}

Tribolium castaneum is a coleopteran insect pest and an emerging insect model in many aspects of molecular and genetic engineering studies because of the availability of its whole genomic sequence ${ }^{16}$, short mode of embryogenesis ${ }^{38}$ as well as highly efficient systematic parental RNAi response ${ }^{39,40}$. Double-stranded RNA (dsRNA) via RNAi mechanism is a defense response used by eukaryotic organisms to guide their cells from invading microflora ${ }^{41}$ which is also employed by T. casteneum.

RNA mediated interference is a biotechnological approach system of gene silencing, which is found to be effective in reverse genetics to investigate the function of a gene in model and nonmodel organisms ${ }^{42}$, and used as an alternative to traditional genetics ${ }^{43,44}$ investigations. RNAi as a biological process is based on degradation of mRNA specific sequence of a specific gene of interest. The degradation is caused by fragments of dsRNA ${ }^{45}$ called short interference RNAs (siRNAs).

The RNAi work in such a way that when the DsRNA enter the cell, it is then being degraded by Dicer-like ${ }^{46}$ protein thereby resulting in small interference RNA (siRNAs). The siRNAs are then further deployed to the RNA-Induced Silencing Complex (RISC) where it recognized and fused to any corresponding single-stranded RNA in the cytoplasm ${ }^{45} 47$, which the lead to the breaking down of the RNA and hence inhibiting its expression. Other activity of RNAi are repressing the activity of transposons ${ }^{48}$, causing virus infection ${ }^{49} 50$ resistance, and the regulation of chromatin structure ${ }^{51,52}$. RNAi is an efficient tool that have been deployed for various reverse genetic studies ${ }^{51,53-56}$.

\section{2.3 CRISPR-Cas9 genome editing:}

CRISPR-Cas system is a novel approach of cleaving the DNA of invading foreign body by the use of RNA guided ${ }^{57}$ nucleases. The system is diverse and found to be used by several bacteria and archaeal organism ${ }^{58}$. It is a complex system that comprises of CRISPR-associated (Cas) genes ${ }^{59}$, noncoding RNAs and collection of repetitive elements ${ }^{60,61}$. Also, found within the array, are a group of repetitive sequences protospacers ${ }^{62-64}$ that are always associated with the Proto Adjacent Motif (PAM) sequence ${ }^{62-64}$ derived from the target DNA.

CRISPR - Cas as a RNA- guided nuclease activity, comprises expression of optimized Cas9 and its RNA component ${ }^{65}$, which then results in several genome editing techniques such as Non Homologous End Joining (NHEJ) or Homology Directed Repair (HDR). The process thus 
involved the redirecting of Cas9 to any target of interest close to the PAM sequence, which subsequently results in DNA Double Strands Breaks (DSB) at the specific loci of interest ${ }^{57}$.

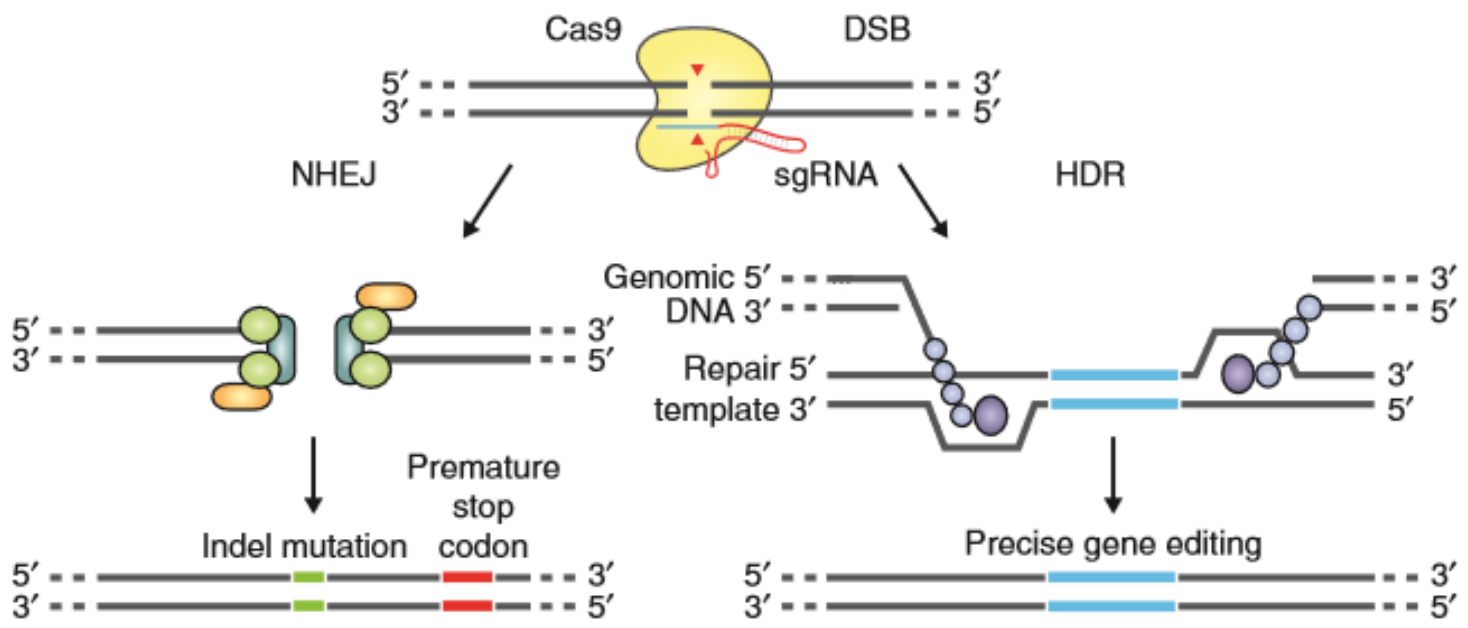

Fig 2: Schematic depiction of Double Strand Break repair which promotes gene editing ${ }^{57}$.

\subsubsection{Site specific recombination:}

This is a transgene integration via genome modification strategy, where specific artificially engineered nucleotide sequence are integrated within the genome of an organism and subsequently targeted via recombination ${ }^{66}$ process. Examples of site specific recombinant systems are, Cre-loxP from bacteriophage P1 ${ }^{67}$, Flp-FRT from Saccharomyces cerevisiae ${ }^{68}$ and phiC31-att from a Streptomyces bacteriophage ${ }^{69}$.

The introduction of recombinase target site term 'docking site", 70 67,71 or 'attP' is achieved using varying molecular biology approach such as transposable ${ }^{24,29}$ element-based transgene transformation or CRISPR-Cas9 ${ }^{60}$, as the first step done during recombination experiment. Afterwards, using complimentary and appropriate molecular biology approach the transgenes of interest carrying attB site is integrated into the generated docking site using phiC31 recombination enzyme ${ }^{72}$. phiC31 integrase is used between attB (carrying transgenes of interest) and attP (introduced as the docking site) sites to integrate a transgene by recombining the attB and attP sites hence, creating attL and attR hybrid junctions ${ }^{73} 69$ in the process, that are no longer accessible to the enzyme after the recombination activity, thereby making the transgenesis stable and efficient ${ }^{69}$. Also because the hybrid sites created are not in insects ${ }^{71}$ in nature, it makes the technique a viable option for the generation of mutants as well as transgenic organisms. One of the strategies used for this approach is called the Recombinase Mediated 
Cassette Exchange (RMCE) with both donor sequence and the docking sites flanked by recombination sites 6973 .

\subsection{Classical Insect Pest Control Measures:}

2.3.1 Physical/Cultural methods: These are the several measures such as proper land sanitation, electromagnetic radiations (http://naldc.nal.usda.gov/download/29407/PDF available 13.06.2016.) and screen ${ }^{74}$ used to limit the access to cultivated crops from attack by insect pests. Other forms of cultural measures used for the control of pests include intercropping ${ }^{75}$, crop rotation ${ }^{76}$, mix cropping ${ }^{77}$, use of resistance varieties ${ }^{78}$ etc.

2.3.2 Biological methods: This is the regulation of insect pest population by the use of natural enemies such as parasite, predators ${ }^{79}$ and parasitoid etc. Example of such practices include, the use of predatory earwig ${ }^{80}$, entomopathogenic nematodes ${ }^{81-83}$, pathogens such as; Metarhizium anisopliae, Beauveria bassiana ${ }^{84,85}$ and Bacillus thuringiensis ${ }^{86,87}$ to suppress insect pest populations.

2.3.3 Chemical methods: These are compounds that can come from either natural origin, such as attractants ${ }^{88}$, repellents ${ }^{88}$, antifeedants ${ }^{89} 90$, pheromones ${ }^{91}$, and hormones or from synthetic compounds. The synthetic compounds ${ }^{92}$ and substances of natural origin ${ }^{93}$ are term as all termed as pesticides. Controlled application of insecticide substances should be used as the last resort for the control of pest population, and selection of appropriate insecticide to use, should be done with consideration of it safe to the environment ${ }^{94}$ and also the problem of resistance development etc.

2.3.4 Integrated Pest Management (IPM): This deals with the management of pests zealously by using their interaction with other living organisms and with the environment ${ }^{95}$. IPM encourages the use of environmentally and human acceptable practices like use of beneficial organisms i.e. natural enemies populations in decreasing insect pest populations 96 rather than indiscriminate use of insecticide that causes damage to both environmental and human health.

In a way to improve the IPM programs, the Area Wide -IPM (AW-IPM) strategy has become a powerful tool for the management of several insect pest for over several years, and this also include the deployment of Sterile Insect Techniques (SIT), which is an environmental-friendly approach that prevents pest status from reaching the economic injury levels. 


\subsection{The Sterile Insect Technique (SIT) and potential improvements:}

The Sterile Insect Technique (SIT) is a birth management strategy that is use to cause infertile mating on conspecific individuals in order to control them ${ }^{97}$. In SIT, reproductively sterile male produce through varying methods are released in large number where they compete with wildtype conspecific male and sought out for conspecific wildtype female individuals for mating, thus blocking the reproduction of the wild females ${ }^{98}$ causing infertile mating when successful. The method, however, is only applied against insect pest that reproduce sexually. Imposition of genetic sterility in the classical approach is usually done by radiation ${ }^{99}$, however another method is through the use of chemosterilants ${ }^{97,100}$ which have been tried, with each of the techniques recording varying level of advantages and disadvantages. Other advantages of SIT is that it is target-specific and environment-friendly ${ }^{101-103}$, and its application is geared towards the reduction in the application of chemical pesticide. 


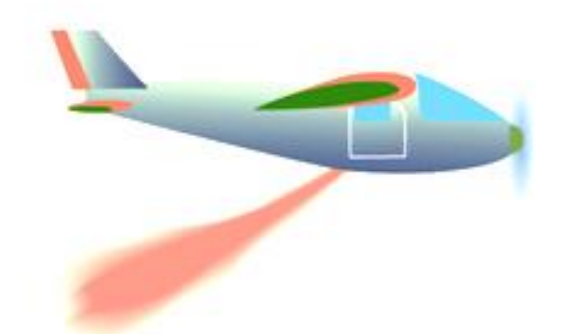

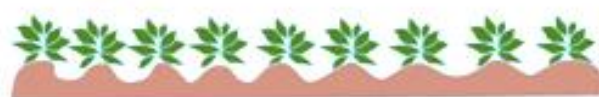

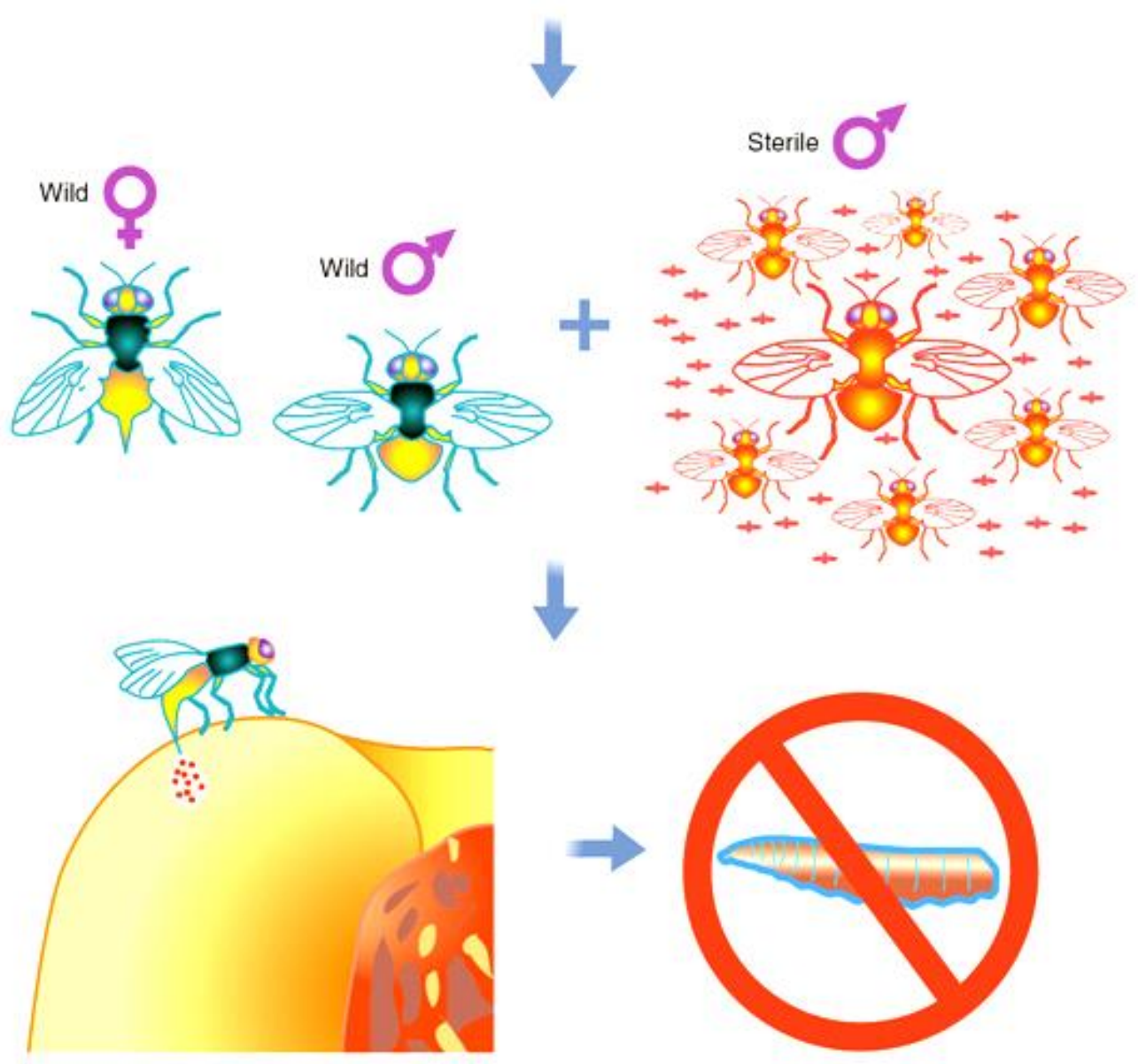

Figure 2: Principle of SIT (Wimmer, 2005)

However, Klassen et al ${ }^{97}$ stated that SIT strategy was originally developed for the New World Screwworm, and studies showed that there is loss in lifespan and competitiveness ${ }^{104}$ when idividuals were rendered sterile by exposure to X-rays in classical approach. However, despite this drawback, the program has been used successfully for managing several pest ${ }^{100,105}$ of human ${ }^{106}$ and agricultural ${ }^{107}$ importance. This success can also be transferred and used for the management of coleopteran pests such as the red palm weevil. 
SIT employed the large release of sterile males to mate and reduce the population of conspecific wildtype individuals ${ }^{97,108}$ via infertile mating, which can be employed to achieve appreciable success in controlling coleopteran insect pest such as the red palm weevil, which is difficult to control because of it cryptic life ${ }^{109}$ and feeding habit which happen inside the tree trunk. Thus, by releasing sterile and competitive males individuals that will search for, and mate with the con-specific wildtype females inside the tree trunk, it should improve the efforts to fight this pest which cannot be access easily by other control measure without attendant health or environmental concerns.

\subsubsection{Suitability of sterile insect technique for the management of insect pests:}

The SIT can be deployed as a substitute to chemical application for the management of suitable insect species. However, the successful application of SIT is based on the ability to produce quality insect for sterilization and release ${ }^{110}$ in large scale. Sterilization in SIT should be done to make sure that individuals developed by either classical genetics i.e. irradiation ${ }^{111,112}$ and chemosterilants or through transgenesis ${ }^{37}$, are good enough to compete in the wild without compromising the integrity of the strain during long-term rearing conditions ${ }^{100,113}$ of one or both sexes.

\subsubsection{Transgenic sterile insect technique to generate conditional male sterile marked insect pests:}

The sterilization by classical genetic SIT strategy ${ }^{112}$ using irradiation generally affect the fitness level of the individuals, because even though the goal is achieved by shredding gametic cells, the somatic cell of the organism are generally affected in the process. The removal of female from the male before sterilization and released has shown to be cumbersome in the classical approach. Also, releasing of only male which is required to be effective ${ }^{101,114}$, and by removing the useless but potentially harmful females from the last mass-rearing step at early embryonic stages ${ }^{100}$ prove difficult in classical approach. Therefore, there is the need for improved strategies to achieved and increase the effectiveness of AW-IPM for SIT approach in the management insect pests. Transgenic approaches can be used to circumvent these problems, but only if it is able to achieve the introduction though biotechnologically approach at least three different types of traits ${ }^{42}$ : firstly, a female specific lethality system that will enable sorting of male-only ${ }^{115-117}$; secondly, introduction of lethality system to replace the classical approach ${ }^{112,118}$; and thirdly, an efficient marker systems ${ }^{119}$ which could provide an effective manner for monitoring and reproductive biology studies. 
In this respect, sterilization ${ }^{104}$, sexing ${ }^{114}$, and marking ${ }^{120}$ can be improved by different strategies and subsequent stabilization of the transgene using biotechnological approach. However, for SIT to be termed as successful for pest control, it has to be species-specific ${ }^{103}$; with no environment or human health concerns as in the case of pesticide application. Also it must be compatible with organic agricultural practices ${ }^{121,122}$, and finally, the organism should be mobile and able to seek out for conspecific mates.

Thus, as laudable as the benefits and efficiency of classical SIT approaches are, there are increased concern about the effects that irradiation and chemosterilants have on the environment and human life and increased on the economics of scale. Different types of transgenic approaches have been adopted over the years, and this include: Transgenic sexing system ${ }^{123,124}$, Reproductive sterility ${ }^{125}$ system and Monitoring ${ }^{126}$ via male sperm marking.

2.4.3 Transgenic sexing for male-only release: The classical genetic SIT process requires the rearing and release of only males in large number who are made sterile by gamma or X-ray irradiation exposure ${ }^{127}$. These released males will actively seek wild con specific females for mating, and because they are not usually released with follow co-released sterile females ${ }^{123}$ it thus eliminate any form of distraction from supposedly co-released sterile females . However, use of radiation affect the fitness of these released males because of the effect of the radiation on their somatic cells. However, the molecular technology approach, by employing the Transgenic Sexing Strains (TSSs) ${ }^{128}$ has being used to solve the drawback of the classical Genetic Sexing Strains (GSSs) such as genetic instability ${ }^{129}$, aneuploidy ${ }^{107}$ etc.

TSSs developed in the model fly Drosophila melanogaster ${ }^{116,130}$ eliminate female individuals via conditional female-specific lethality ${ }^{115,123}$ by binary conditional system ${ }^{37}$ at the embryonic stage of development. Recently, TSSs has also been established for diverse Tephritid pest species ${ }^{123,124,131}$.

2.4.4 Transgenesis as a replacement to radiation sterilization prior to release: An insect is termed sterile if it cannot produce viable offspring as a result of an appropriate treatment ${ }^{121}$ application, without however not having adverse effect on the fitness of the treated individual. At the inception of SIT programs, treatments using chemosterilants ${ }^{132}$ and radiation ${ }^{118}$ are employed for insects sterilization. The 2 treatments achieved similar ${ }^{100}$ results, but since most of the chemosterilants which were used for the treatment are reported to have adverse human and environmental effects, their use was stopped, with the retention of ionizing radiation 
sterilization ${ }^{133}$ as the preferred approach. Therefore, the insects prior to release are exposed to Cobalt-60 or Caesium-137 in irradiators, which also has some disadvantages by sometimes exposing the workers to the radiation ${ }^{132} 133$. Also, despite the effectiveness of the strategy and it low level of application, there are reported cases of resistance development that requires higher doses to be applied. As a substitute to irradiation, transgenesis has provided a great potentials as a means of addressing the negative effect of radiation because: by using transgenic system death can be induce ${ }^{134}$ at embryonic stage using recombinant DNA, and thereby achieving the same result without the need for irradiation.

Transgenic systems have been designed in such a way that the individuals can be reared efficiently with no effect to their fitness level. The system is designed in a binary and conditional manner, where tetracycline is added or removed to their food ${ }^{135}$ in order to regulate the system. Where tetracycline is added to the food of the animal in a tet-off system, the tetracycline trans-activator (tTA) is prevented from activating the desired target gene expression ${ }^{135}$ because it cannot bind to the Tetracycline Responder Element (TRE), but if tetracycline is withdrawn, the synthetic tTA initiates the target gene to be expressed, because the tTA bound effectively to the TRE therefore activating target gene expression ${ }^{136}$, as reported in Drosophila melanogaster ${ }^{137}$.

Horn and Wimmer ${ }^{138}$ reported a different approach to reproductive sterility in D. melanogaster where sterility was induced by combining the transgenes that caused embryo-specific lethality. This principle has now been transferred to the Tephritid pest species, Ceratitis capitata, ${ }^{134}$ and Anastrepha suspensa ${ }^{124}$ via isolating species-specific blastoderm-expressed genes and their respective promoters/enhancers.

\subsubsection{Monitoring of released transgenic insects:}

Monitoring is an integral part of any SIT approach, where as in the classical approach fluorescent dye are used in the dusting of pupae before they are released to the environment 139. The fluorescent dye help in distinguishing release sterile individuals from wildtype individuals during monitoring. As laudable and effective as the system is, it posit some disadvantages amongst which include: they are costly, error prone ${ }^{140}$, and it also leads to development of false positives because the florescent dyes can be rubbed off from sterilized dusted marked individuals to wild type ones ${ }^{140}$ since they are bodily applied. Also, the reproductive biology of the insect cannot be studies using fluorescent dyes. 
To circumvent the disadvantages enumerated above, a dominant, heritable fluorescent marker such DsRed and EGFP can be conferred for marking of sperm through transgenesis ${ }^{35,141-143}$. Also, the problems of false positives can be effectively avoided since it's the sperms that are marked and not bodily applied as in the case of fluorescent dye observed in the classical SIT approach.

Transgenic marking of insect sperm using fluorescent protein has been successfully achieved for Anopheles stephensi ${ }^{142}$, Aedes aegypti ${ }^{144}$, C capitata ${ }^{126}$ species etc., by using their spermatogenesis specific beta2-tubulin gene upstream region to drive the expression of fluorescence proteins in their sperm. Fluorescence sperm marking by transgenesis thus also allow for sexing, as well as screening transgenic sperm marked males from wild-type individuals. Moreover, sperm-marking facilitates the identification of inseminated wild type females and therefore improving the level of monitoring in an SIT programs, and for the study of reproductive biology.

\subsubsection{Transgenic insect stability:}

One of the major concerns of transgenic insect research is the ability to maintain the stability of the transgenes within the insect after released ${ }^{145}$. The inability to maintain the stability of the transgenes in the genome after release can cause serious reduction in the effectiveness of the biocontrol program ${ }^{145}$. Several success of transgene stabilization in D. melanogaster ${ }^{146,147}$, C. capitata $^{148}$, and piggyBac-mediated transgene insertions ${ }^{134}$ in C. capitata and modification by site-specific recombination system ${ }^{149}$ have being reported. ${ }^{134}$.

Another concern is due to the fact that the transposable elements utilized for genetic transformation in transgenesis are mostly insect based ${ }^{150}$, thereby providing a situation whereby "jumping" activities in the field is possible. This problem however, have being circumvented by the use of defective transposons insertion ${ }^{121}$. 


\subsection{Objectives:}

Coleopteran insects are the largest order in the class Insecta, they represent about a quarter of all insect species described and found to inhibit all niches. Some of them are found to be causing considerable loss to not only human health but also agricultural crops and animal losses. Several control measures have been employed for the management of coleopteran pests ranging from mechanical/physical, chemical, biological control measures. However, as laudable as the measures are, they are still ineffectual as some become more cumbersome and leads to development of resistance against the chemicals that are hitherto effective in controlling them. Insect transgenesis approach based on male-only sex-separation, reproductive sterility, and sperm marking for monitoring as envisaged in this research project are possible strategies for overcoming some of the observed disadvantages of the conventionally used practices. The SIT approach is a cheap measure of pest management practice, that has been employed and found effective in some dipterans i.e. Ceratitis capitata, Drosophilidae and several mosquitoes species. However, there is paucity of information about the use of the transgenesis technique in coleopteran insect pest. However, effort of developing classical Sterile Insect Technique (SIT) some coleopteran insect pest such as RPW $R$. ferrugineus ${ }^{151}$ have been attempted.

Thus as a prove of principle in establishing the transgenic techniques i.e. Sperm Marking, Female Embryonic Lethality (FELS), sexing for males only release and functionally testing the techniques will be employed in the Red Flour Beetle (RFB) Tribolium castaneum the "ModelColeopteran" because of the availability of its full genomic sequence, availability of transcriptomic data, short generation time, ease of rearing and availability of comprehensive expression system. 
In this study, my particular objectives are;

1) Identify coleopteran genes that are testis specific or cellularization specific as tools for Sterile Insect Technique (SIT).

2) generate and test a transgenic sperm marking system.

3) Test controllable binary expression systems in Red Flour Beetle.

4) Generate transgenic lines for SIT approaches in beetles. 


\section{RESULTS}

The results of this thesis are presented in a manuscript format comprising of the following sections, preceded by a description page describing

- Aim of the manuscript in relation to the thesis as a whole

- Authors contribution to the work

- Manuscript status

- References cited at the end of the particular chapter

References cited in the general introduction and the general discussion are found at the end of the discussion (thesis chapter $\mathbf{6}$ ) 


\subsection{Transgenic sperm marking to enhance monitoring of coleopteran insect pests:}

In this chapter, we provide a proof of principle of transgenic sperm marking in coleopteran insect pests. Using the spermatogenesis specific B2-tubulin gene, we drive either DsRed or EGFP fluorescent proteins in the sperm of the Tribolium castaneum male individuals. The marked sperm individuals thus provided us with an efficient tool for the assessment of various monitoring practices, determination of the competitiveness of the transgenic individuals to wildtype conspecific individuals as well as reproductive biology studies in twice mated Tribolium casteneum wildtype female. This is a first step in establishing a Sterile Insect Technique (SIT) targeting coleopteran insect pests, which is the largest order in the class insecta that comprises more human and agricultural insect pests than any order of insects.

ISAH, Musa Dan'azumi, Bibi Atika, Stefan Dippel \& Ernst A. Wimmer Authors contribution to the practical work

ISAH, Musa Dan'azumi: Conceived and design the experiment, conducted all the other experiment and analysis. Wrote the manuscript.

Bibi Atika: Crossings and screening of positive sperm marked individuals and microscopic analysis of the spermatheca of inseminated females

Stefan Dippel: Conceived and design the experiment and assisted in the molecular cloning of the sperm marking constructs

\section{Status: Work in progress}




\section{Transgenic sperm marking to enhance monitoring of coleopteran insect pests:}

ISAH, Musa Dan'azumi ${ }^{1,2}$, Bibi Atika ${ }^{1}$, Stefan Dippel $^{1}$ \& Ernst A. Wimmer ${ }^{1 *}$

1) Department of Developmental Biology, Johann-Friedrich-Blumenbach-Institute of Zoology and Anthropology, Göttingen Center for Molecular Biosciences, Georg-August-University Göttingen, 37077 Göttingen, Germany.

2) Department of Crop science, Faculty of Agriculture, Wildlife and Forestry Resources Management, University of Calabar, P.M. B. 1152, Calabar, Cross River State, Nigeria.

* Corresponding Author: E. A. Wimmer. TEL. +49 5513928666

E-Mail address: ewimmer@gwdg.de.

\section{Abstract:}

Tribolium castaneum belongs to the order coleoptera and has been employed in the study of gene function, for both evolutionary and developmental biology question apart from Drosophila melanogaster. The Sterile Insect Technique (SIT), is a specie specific and environmentally friendly birth management practice geared towards efficient control of pest population, which will effectively substitute the deleterious application of chemical control practices.

Monitoring practices such as the use of pheromones traps and fluorescent dyes among others have been adopted for studying infestation levels of several coleopteran and other insect pests to identify the insipient insect pest situation for the deployment of various pest management strategies. The use of spermatogenesis-specific 32 -tubulin promoter driving a fluorescent marker in the sperms is an efficient substitute to the error prone fluorescent dye application employed in classical genetic control strategy for monitoring in an SIT program. The system has been used to engineer Ceratitis capitata, Anopheles gambiae, Aedes aegypti and Anastrapha suspensa. The transgenic sperm marked individuals can also be used for sexing for male only releases and for the study of reproductive biology. Our objective in this section is to achieve transgenic sperm marking for Tribolium castaneum, by using T. castaneum spermatogenesis-specific 32 -tubulin promoter to drive a fluorescent protein in the sperm, which can also be applied in functional large-scale SIT activities.

As a proof of principle, we generated fluorescent marked sperm T. castaneum strains, and using the strains we evaluate assays for fitness, mating ability, sperm competition, and for its prospect in an easy and stable monitoring SIT programs. The sperm marked individuals were used to determine more detailed information on reproductive biology of T. castaneum. 
Key words: Tribolium castaneum, Sperm marking, Pest Management, Monitoring, Insect transgenesis

\section{BACKGROUND:}

The Red Flour Beetle Tribolium castaneum belongs to the class Insecta and order Coleoptera ${ }^{1}$, and about 380,000 coleopterans insect species have so far been reported ${ }^{2}$. Beetles constitute a quarter of all animal species in the world ${ }^{3}$, and $40 \%$ of the class insecta. Their successes could be attributed to the possession of diverse anatomical structures and are found in different ecological ecosystems ${ }^{4}$.

Tribolium castaneum is a very important family in the order coleoptera, they are found in all continents and affecting diverse agricultural products especially stored grains ${ }^{5}$. They are the very model for higher diploid animals ${ }^{6}$ for genetic studies. They possess 10 chromosomes pairs, thus making them a simple and appropriate organism for genomic studies ${ }^{6}$. Their short generation life span, ease of rearing in the laboratory, small size has also provided the insect as a good model for the studies of population genetics, several developmental and evolutionary biology questions ${ }^{7}$ among others. Moreover, with a fully annotated genome and available toolbox for genetic studies, Tribolium further provides a means for understanding both evolutionary and biological processes in this most diverse animal taxon.

The Red Flour Beetle being a cosmopolitan polyphagous organism causes severe economic damage to some stored products ${ }^{8}$, and has being managed using several control measures such as application of chemicals, ${ }^{9} 10$ among other. However, the indiscriminate use of chemicals has resulted into several issues such as human and environmental health problems, buildup of resistance ${ }^{11}$ etc. Other forms of control measures employed for the management of this insect are: biological control strategies such as the use of fungi, bacteria 12 etc., within Integrated Pest Management practices.

Monitoring practices using pheromones ${ }^{13}$ and fluorescent dyes is an integral part of pest management strategies and have been adopted for studying insipient infestation levels of several coleopteran insect pests ${ }^{13}$ in both field and for some stored product insect pests. Paternity and sperm precedence assay using phenotypic genetic markers ${ }^{14} 15$ in Tribolium ${ }^{15}$, carrion ball roller beetle ${ }^{16}$, as well as allozyme markers ${ }^{17}$ in Colorado potato beetles, 
short time sperm storage assay in Tribolium etc., have being reported as pest management strategies.

The Sterile Insect Techniques (SIT), is a birth management practice geared towards efficient control of pest population ${ }^{18}$. SIT can be achieved via a classical genetic approach which involves the use of irradiation for sterility ${ }^{19}$, sexing using visual markers such as pupal colour 202122 or temperature sensitive lethal alleles ${ }^{20}$ and heat shock, or the use of fluorescent dyes ${ }^{23}$ for monitoring which are mostly expensive and largely non environmentally friendly and reliable. However, several biotechnological transgenic approaches have being adopted to replace the expensive, labour intensive, error prone ${ }^{24}$ and environmentally unfriendly monitoring technique based on powdered dyes used for the classical genetic SIT strategy, by utilization of recombinant DNA approaches to provide alternatives in body and sperm marking in An. gambiae and Ae. aegypti ${ }^{25}{ }^{26}$, C. $_{\text {capitata }}{ }^{27}$, Anastrapha suspensa ${ }^{28}$.

Transgenic marking of sperm using flourescent proteins thus provides an excellent alternative 27 to fluorescent dyes used in classical approaches. This enhances the effectiveness in monitoring, which is a critical part of SIT, because it helps in discriminating wildtype individual from released individuals. It also allows for fast and accurate evaluation of mated females because the marked sperm can be visualize in the female spermatheca as reported in C. capitata $^{29}$ and for sexing males from females ${ }^{25}$.

In this study we present a proof of principle of transgenic sperm marking in Tribolium castaneum (Coleoptera: Tenebrionidae) by recombinant DNA, and also uses it as a tool in mating assays between transgenic and wildtype individuals to analyze for paternity and sperm competitiveness over a prolong period of time, as well as for reproductive biology assay. 


\section{Results:}

\section{Isolation of the $\beta 2$ tubulin gene of Tribolium castaneum .}

To establish a sperm marking system that will be used to drive a sperm specific expression in Tribolium castaneum testis, we needed to isolate the potential upstream of the Tc- $\$ 2$ tubulin gene. We thus determine the spermatogenesis specific Tc 009035 (GENBANK accession number XP_969993) B2-tubulin gene for T. casteneum using a literature search ${ }^{30}$. Using the gene $T c$ number ${ }^{30}$, we did a query search in the $T$. castaneum Tcas 5 (http://bioinf.unigreifswald.de/gb2/gbrowse/tcas5/) ${ }^{31}$ genome database to identify the putative full sequence of the gene. The gene Tc 009035 B2 tubulin gene is coding for 452 amino acids and made up of 1347bp Open Reading Frame (ORF) which is bordered with 121bp 5'UTR and 258bp 3'UTR and interrupted by a single 46bp intron (Fig: 1a).

To determine the regulatory elements that are needed for effective and efficient gene expression in the $T$. castaneum testes, we tried to identify the necessary conserved sperm specific regulatory elements: ßt Upstream Element 1 (ß2UE1 )(ATCGTAGTAGCCTA), ß2UE2 (GAACATTC), the 7bp Initiator sequence (inr) (TTCAGTT) important for TATA-less promoters in the downstream element and B2tDE1 (AAAATTATACGTTTAAAT) to Drosphila melanogaster ${ }^{32}, D$. suzuki ${ }^{33}$ in $T$. castaneum. However, these elements could not be identified in the tribolium genome and thus they might therefore be dipteran specific.

\section{Generation of sperm marking constructs and strains using $\beta 2$ tubulin promoter region.}

To generate sperm marked individuals, we cloned sperm marking constructs by fusing a $1 \mathrm{~Kb}$ Promoter/Enhancer $(\mathrm{P} / \mathrm{E})$ region of the $T c-\beta 2 \mathrm{t}$ gene to either a fast folding fluorescent proteins EGFP (Fig 1c) to express green fluorescent protein in the testis, or DsRed (Fig 1b) to express red fluorescent protein in the testis. To generate the transgenic strains, we performed piggyBac germline transformation microinjection.

We identified 18, $F_{1}$ transgenic offspring from $4, G_{0}$ parents (Table 1) expressing DsRed marked sperms (Fig 1b) and in a like manner $4, F_{1}$ transgenic offsprings from 3, $\mathrm{G}_{0}$ parents (Table 1) expressing EGFP (Fig 1c) marked sperms were identified. However, the male wildtype individuals have no fluorescence's that could be identified (Fig: 1a) in their testis when compared to the transgenic strains. 


\section{Transformation efficiency:}

To determine the transformation efficiency of the 2 injected piggyBac constructs i.e.

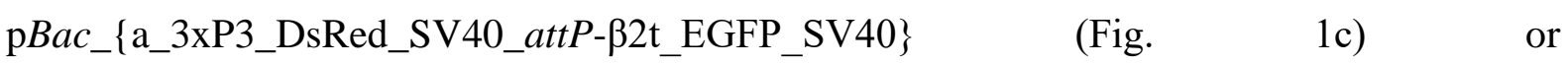
pBac_\{a_3xP3_EGFP_SV40_attP_B2t_DsRed_SV40\} (Fig: 1b) sperm marking constructs mixed with mhybase helper plasmid ${ }^{34}$ in the appropriate concentration. Of the 1000 embryos injected with pBac_\{a_3xP3_DsRed_SV40_attP-32t_EGFP_SV40\} (Fig: 1c) construct, a total of 139 embryos developed into larvae, with 77 survived to the pupal stage $\left(\mathrm{G}_{0}\right)$ subsequently. We then singly crossed each injected pupa to corresponding wildtype (WT) of the opposite sex, resulting in a $7.3 \%$ transformation rate (Table 2). In a like manner, when the pBac_a\{3xP3_EGFP_SV40_attP_B2t_DsRed_SV40\}(Fig: 1b) construct was injected into 1000 embryos, 210 hatched larvae are recorded among which 107 survived to pupal stage $\left(\mathrm{G}_{0}\right)$. we subsequently singly crossed each to its corresponding wildtype (WT) of the opposite sex with a transformation rate of $21.8 \%$ (Table 2).

\section{Molecular characterization of sperm marking lines (Inverse PCR of genomic DNA)}

To examine the single integration of the piggyBac-mediated integrations at the TTAA canonical target sites of the generated sperm marked (i.e. constructs pBac_\{a_3xP3_DsRed_SV40_attP-ß2t_EGFP_SV40 $\}$ and pBac_\{a_3xP3_EGFP_SV40 _attP_B2t_DsRed_SV40\}(Supplementary information 3a and 3b) strains. We recovered by inverse PCR the genomic DNA sequences flanking 5' and 3' $p B a c$ insertions positions in the genome (Supplementary information 3).

\section{Expression of fluorescence protein in testis and single sperms and sperm transferred to} the spermatheca of females.

The testis of the wildtype and transgenically sperm marked males were dissected and analyzed for the detection of sperm individuals. The results show that the testis of the transgenic individuals marked with either DsRed (Fig: 2b) or EGFP (Fig: 2c) showed fluorescence depending on which transgenic sperm marked individual was viewed, but no fluorescence was observed from the wildtype dissected testis (Fig: 2a).

Also, single sperms from testis obtained from transgenic males pBac_\{a_3xP3_EGFP_SV40_attP_B2t_DsRed_SV40\}\&pBac_\{a_3xP3_DsRed_SV40_attP32t_EGFP_SV40\} shows either DsRed (Fig: 2b) or EGFP (Fig: 2c) fluorescence respectively 
which we found to be confined to the sperm tails, which however no fluorescence could be observed in the single sperm (Fig: $2 \mathrm{a}$ ) of wildtype males.

In a similar manner, to determine whether if the single transgenically marked sperm can be observed in the spermatheca of a wildtype female, we analyzed the spermatheca of a singly mated wildtype female with either DsRed or EGFP male sperm marked individuals respectively. In addition, the testis of the DsRed or EGFP sperm marked individuals was also analyzed. The fluorescence observed in the dissected spermatheca of the wildtype females mated with either the DsRed (Fig: 2b) or EGFP (Fig: 2c) sperm marked males proved that the ß2t $\mathrm{P} / \mathrm{E}$ region contain the necessary elements responsible for driving the expression fluorescent protein in the sperms. No fluorescence was however observed from the spermatheca of wildtype female mated with wildtype males (Fig: 2a) or the wildtype single sperm. Therefore, the ability to detect the marked sperm in the spermatheca of the mated wildtype female further proved that it can be deployed for monitoring in an AW-SIT program for coleopteran insect pests similar to medfly ${ }^{27}$

\section{Evaluation of pBac a_3xP3_EGFP-SV40\{attP-B2t_DsRed_SV40\} and pBac a_3xP3- DsRed-SV40 \{attP-B2t_EGFP_SV40\} generated lines}

\section{I) Sequential sperm competition assay:}

To determine the sperm utilization and competition of sequentially twice mated female, we designed a laboratory assay were differently transgenic marked sperm individual males with either DsRed (M1_F1 and F1_M1) or EGFP (F37_F1, F37_M2) mated sperms were sequentially mated with wildtype vermillion ${ }^{\text {white }}\left(v^{w}\right)$ Tribolium females. The result of the experiment show that no significant difference in the number of progenies was observed when F37_M2 individuals were used as the second inoculated males; F1_M1 x F37_M2, M1_F1 x F37_M2 and also when used as the first inoculated male in F37_M2 x F1_M1(Supplementary 1 and 6). Also, no significant difference in progenies for M1_F1 x F37_F1 cross was obtained. The result further elucidates the fact that the females start utilizing the sperms of the second mated male after which the first male sperms is used again (Supplementary 1 and 6). Thus, it suggests that the first male sperm are not completely replenish or lost from female reproductive organ but rather stored in the spermatheca for further use. 


\section{II) Out-crossing and repetition of sequential sperm competition assay to determine any bottleneck effect:}

A test was set up to outcross the generated homozygous of M1_F1, F1_M1 (pBac \{a_3xP3_EGFP-SV40_attP-ß2t_DsRed_SV40\}) and F37_F1, F37_M2 (pBac \{a_3xP3DsRed-SV40_attP-B2t_EGFP_SV40\}) individuals. We singly outcrossed the homozygous individuals to wildtype $v^{w}$ of the corresponding sex to make them heterozygous, and subsequently following several crosses we make them homozygous again to ensure that a potential bottleneck effect that might have effect on the performance of the different strains were diluted, and the sequential sperm competition assay was repeated

The result of the experiment showed that there is no significant difference when F37_M1 strain was used as the second inoculated male in a F1_M1 x F37_M1, M1_F1 x F37_M1 combination. Also, when F37_M2, and M1_F1 were used as the first inoculated male i.e. M1_F1 x F37_M2 and F37_F1 x M1_F1 respectively no significant difference was observed. The result further elucidated the fact that the females start utilizing the sperms of the second mated male after which the first male sperms is used again (Fig: 3 and Supplementary: 6). The result further suggest that the first male sperm is not completely replenished or lost from the female spermatheca but rather stored for subsequent use.

\section{III) Sperm dynamic and distribution pattern in twice-mated females fertilization chambers}

To determine the dynamic and distribution of sperms within the reproductive chambers of a once or twice mated wildtype females by 2 different sperm marked with EGFP and DsRed male individuals. The spermatheca of a singly mated female with either EGFP or DsRed sperm marked males was observed and, the sperms appeared to be distributed evenly within the fertilization structure (Fig 6a and 6b). However, no fluorescence was observed in the control were wildtype male was mated with the wildtype female. In the twice mated female, the dynamic of the sperm within the fertilization chamber 2 days after the second male copulation marked with either EGFP or DsRed sperm, showed that the sperm of first inoculated male was seen to be widely distributed within the spermatheca but however, encapsulated by the sperm of the second mated males.

However, ten days after the mating with the second marked sperm males, the sperms of both first and second within the spermatheca of the mated female appeared homogenously 
distributed (Fig 4a and 4b) with no clear localization of either the first or second mated males sperms.

\section{IV) Competition assay between different transgenic strains:}

To evaluate the paternity of offsprings sired by 2 different Tribolium male individuals in laboratory experiments. We used M1_F1, F1_M1 (pBac \{a_3xP3_EGFP-SV40_attPB2t_DsRed_SV40\}) and F37_F1, F37_M2 (pBac \{a_3xP3-DsRed-SV40_attPB2t_EGFP_SV40\}) homozygous transgenically DsRed and EGFP sperm marked male individuals respectively, which are mated with wildtype Tribolium $v^{w}$ females of the same age.

The results of the experiment after 4 days of inoculation period showed that no significant difference in number of progenies sired in cross of M1_F1 x F37_F1 strains, while the other combinations showed that the EGFP sperm marked individuals sired significantly more progenies than the DsRed sperm marked individuals (Supplementary Figure 2a and 4).

However, the results of progenies sired after the mated females were removed after 4 days, and kept for further 21 day on a new flour showed that significantly higher number of individuals were sired by F37_F1 EGFP sperm marked strain in M1_F1 x F37_F1 combination than sired by the DsRed sperm marked strains. While no significant difference in offspring numbers was observed in the other combinations (Supplementary Figure 2b and 4).

\section{V) Competition assays of transgenic generated individuals against wildtype.}

To assess the mating/fitness ability of homozygous transgenically marked sperm strains with either DsRed or EGFP protein against any form of observable fitness problems due to the foreign constructs introduced in their genome, as against the wildtype individuals with no foreign constructs, and to test their ability to compete favourably with the wildtype individuals in a laboratory situation. After outcrossing to address any potential bottleneck effects as stated above and repeating the experiment, we first performed a Chi test of homozygosity analysis for statistically homozygosity of the transgenic strains. The results showed that, the number of progeny sired by transgenic individuals in normal competition and other competition, except F37_M1 in relax and high competition are not significantly different from the number of progenies sired by the wildtype individual (Fig: 5a, b and c). Conversely, significantly more progenies were sired by wildtype males in M1_F1 relax and F37_F1 high transgenic males. 
Furthermore, the results of the other strains in normal $(1 \times 1 \times 1)$ and high $(2 \times 2 \times 1)$ competition, showed mean significant difference in the fitness and performance level between F1_M1 and F37_F1strains from the wildtype individuals (Figure 5a and 5c, Supplementary 3). However, in different ratio term as the relax $(1 \times 1 \times 2)$ experimental set up, the result showed that the wildtype individuals outperformed all the transgenic strains (Figure 5b, Supplementary 3).

More so, the percentage of progenies sired by transgenic male individuals against wildtype in the normal, relax and high competition assay was determined. The progenies sired by transgenic male individual F37_M2 with EGFP marked sperm in both relax and high competition are not significantly different (Fig: 6) from the individuals sired by the wildtype individual. This is indicative that no negative load affects the fitness capability of the F37_M2 transgenic strain in the assay. 


\section{MATERIALS AND METHOD:}

\section{Beetle stock and rearing:}

Red Flour Beetle (RFB), Tribolium castaneum vermillion ${ }^{\text {white }}\left(v^{w}\right)$ strain was used in all experiments unless otherwise stated. Beetle stocks were fed on full grain flour supplied with $5 \%$ yeast powder in a 4.5liter square plastic boxes $(15 \mathrm{~cm} \times 15 \mathrm{~cm} \times 20 \mathrm{~cm})$ with ventilation mesh grilled on the lid and were kept at $32^{\circ} \mathrm{C}$ with constant light ${ }^{1}$.

\section{Microinjection protocol:}

Germline transformation was conducted as described ${ }^{35}$. Wild type $v^{w}$ strain was placed on white flour for 1 hour after which the embryos were sieved out and kept for additional hour at room temperature. The embryos were washed twice with $1 \%$ Klorix solution ${ }^{34}$ and rinsed with clean tap water.

Embryonic injection was done by FemtoJet ${ }^{\circledR}$ Microinjector (Eppendorf, Hamburg, Germany). Needles for the microinjection was made from $10 \mathrm{~mm} \times 1 \mathrm{~mm}$ borosilicate capillaries by pulling them with a P-2000 micropipette puller (Sutter Instrument, Novato USA) with the following settings: Heat $=350$, Fil $=4$, Vel=50, Del=225, PUL $=150$. The pulled needles were opened and sharpened using a Bachofer Laboratoriumsgeräte beveller (Reutlingen, Germany).

After injection, the embryos were transferred onto an apple agar plates and sealed in a plastic box, which was then kept in an incubator set at $32^{\circ} \mathrm{C}$ for 2 days. After 2 days, the lid of the box containing the injected embryos were opened and kept until hatching. Hatched larvae were picked with a hair thin brush individually and placed on full wheat flour until pupation. After pupation, the pupae $\left(\mathrm{G}_{0}\right)$ were sexed and individually crossed to 3 non-injected individuals of the opposite sex.

\section{Isolation of $32 \mathrm{t}$ promoter region to generate sperm marking constructs.}

To establish a sperm marking system in $T$. castaneum, $1 \mathrm{~Kb}$ beta 2 tubulin ( $(32 \mathrm{t}$ ) spermatogenesis specific Promoter/Enhancer (P/E) region of Tc 009035 (GENBANK accession number XP_969993) ${ }^{30}$ was amplified from San Bernardino (SB) genomic DNA (SB-gDNA). The SB-gDNA was extracted using the NucleoSpin ${ }^{\circledR}$ DNA Insect kit (MachereyNagel GmbH $\alpha$ Co. KG, Düren, Germany). Polymerase Chain Reaction (PCR) by using MID\#4/MID\#7 primer pair in a $50 \mu 1$ reaction consisting of water $28 \mu 1$, gDNA $2 \mu 1,5 x$ Phusion buffer $10 \mu 1$, Phusion polymerase $1 \mu \mathrm{l}$, dNTPs $5 \mu 1$, forward primer $2.5 \mu 1$ and reverse primer 
$2.5 \mu \mathrm{l}$ in a program of $2 \mathrm{~min}$ at $98^{\circ} \mathrm{C}, 30 \mathrm{sec}$ at $98^{\circ} \mathrm{C}, 30 \mathrm{sec}$ at $68^{\circ} \mathrm{C}, 2 \mathrm{~min}$ at $72^{\circ} \mathrm{C} 35 \mathrm{cycles}$, $10 \mathrm{~min}$ at $72^{\circ} \mathrm{C}$ were conducted in a thermocycler. The amplified fragment was run on $1 \%$ agarose gel and a distinct single band was excised and purified using the NucleoSpin ${ }^{\circledR}$ Gel and PCR Clean-up Kit (Macherey-Nagel GmbH $\alpha$ Co. KG, Düren, Germany). The amplified and purified fragment was fused to a either a fast folding fluorescent proteins EGFP ${ }^{36}$ or Discosoma Red fluorescent protein (DsRed) ${ }^{37}$.

\section{Cloning of B2t promoter region to generate sperm marking constructs in a two-step procedure.}

\section{Shuttle vector:}

The first cloning step was conducted using a shuttle vector where, a pSL_af_tTA-SV40 plasmid and the amplified $1 \mathrm{~Kb} \beta 2 \mathrm{t}$ spermatogenesis specific Promoter/Enhancer $(\mathrm{P} / \mathrm{E})$ region were both cut by NcoI/ClaI and joined by ligating their cut ends after removing tTA-SV40 from the pSL plasmid to generate pSL_af_ $\beta 2 \mathrm{t}$. Afterwards, 5 microliters each of attP forward and reverse oligos were pipetted and mix with $90 \mu \mathrm{l}$ of water and placed in a heat block set at $95^{\circ} \mathrm{C}$ for 5 minutes, after which the heat block was switch off and ramp to room temperature to anneal the oligos. The pSL_af $\{\beta 2 \mathrm{t}\}$ was cut using EcoRI/NcoI, and subsequently the attP annealed oligos was ligated upstream of the $\beta 2 \mathrm{t}$ using the cut EcoRI/NcoI pSL_af_ $\beta 2 \mathrm{t}$ end to generate pSL_af_attP- $\beta 2 \mathrm{t}$.

The generated pSL_af_attP- $\beta 2 \mathrm{t}$ was used to generate 2 constructs for expressing either DsRed or EGFP marker proteins in the testis to be driven by the $\beta 2 t$ spermatogenesis specific promoter. A $1 \mathrm{~Kb}$ DsRed-SV40 or EGFP-SV40 was obtained by either cutting pSL_DsRedSV40af for DsRed-SV40 or amplifying EGFP-SV40 with MID\#103/MID\#104 primers from pSL_EGFP -SV40af ${ }^{38}$. The DsRed-SV40 and EGFP-SV40 fragments were cut with ClaI and HindIII and each ligated independently downstream of attP- $\beta 2 \mathrm{t}$ with $\mathrm{pSL} \_$af $\{$attP- $\beta 2 \mathrm{t}\}$ also cut by ClaI/HindIII. The cut fragments and plasmid where ligated together to generate 2

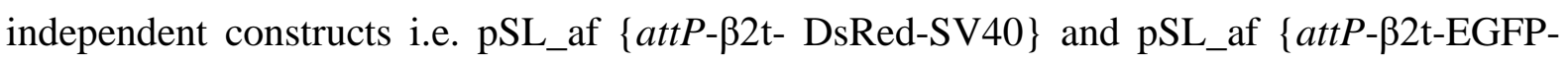
SV40\}.

\section{Transformation vector:}

Finally, the fragments $a t t P \_\beta 2 \mathrm{t} \_$DsRed_SV40 and $a t t P \_\beta 2 \mathrm{t} \_$EGFP_SV40 were excised out of

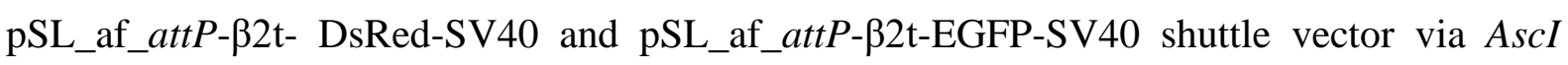
digestion and inserted independently into AscI-cut pBac_\{3xP3_EGFP_SV40af $\}^{38}$ and pBac_ 
\{3xP3_DsRed_SV40af\} ${ }^{39}$ transformation vectors carrying fluorescent transformation eye markers, respectively. The cut fragments were run on $1 \%$ agarose gel and distinct single bands were excised and purified with the NucleoSpin ${ }^{\circledR}$ Gel and PCR Clean-up Kit (Macherey-Nagel $\mathrm{GmbH} \alpha \mathrm{Co}$. KG, Düren, Germany). The fragments were ligated to the transformation vectors to finally generate pBac_a $\left\{3 x P 3 \_D s R e d \_S V 40 \_a t t P-\beta 2 t \_E G F P \_S V 40\right\}$ (Fig 1c.) and pBac_a $\left\{3 x P 3 \_E G F P \_S V 40 \_a t t P \_\beta 2 t \_D s R e d \_S V 40\right\}$ (Fig 1b) sperm marking constructs.

Germline transformation of sperm marked lines with pBac_a $\{3 \times$ P3_DsRed_SV40_attP-

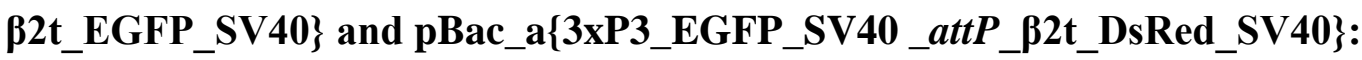

The constructs pBac_a $\{3 \times P 3$ EGFP_SV40_attP_ß2t_DsRed_SV40 $\}$ and pBac_a

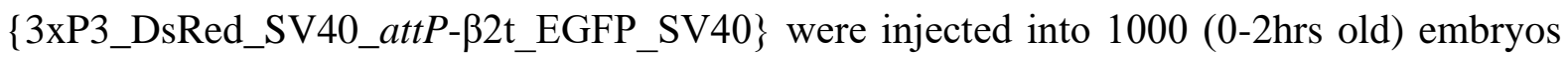
each using the following concentrations: piggyBac constructs $500 \mathrm{ng} / \mu \mathrm{l}$ in injection buffer (i.e. $5 \mathrm{mM} \mathrm{KCl}, 0.1 \mathrm{mM} \mathrm{KH} 2 \mathrm{PO} 4,0.1 \mathrm{mM} \mathrm{NaH} 2 \mathrm{PO} 4 \mathrm{pH} 6.8$ ) along with $300 \mathrm{ng} / \mu 1$ helper plasmid mhypBase ${ }^{34}$ into embryos of the $v^{w}$ strain.

\section{Transformation efficiency:}

To generate transgenic individuals, the 2 generated sperm marking constructs (Fig BI) and (Fig AI) were injected into 1000 tribolium $v^{w}$ embryos each. The developing larvae were collected and kept on full grain until to pupal stage $\left(\mathrm{G}_{0}\right)$ which were counted and subsequently singly crossed to it corresponding wildtype (WT) individuals after being sexed. $\mathrm{G}_{1}$ individuals for possible transgenic were screened for DsRed fluorescence (Fig 1ciii) or EGFP fluorescence (Fig biii) in the eyes in both males and females driven by $3 \times \mathrm{P} 3$ promoter. The transformation rate of the injection was analyzed (Table 2).

\section{Strategy for the development and identification of homozygous of transgenic Sperm marking strains in Tribolium castaneum}

Homozygous individuals were obtained by outcrossing transgenic individuals with wildtype $\mathrm{v}^{w}$ of the corresponding sex and the heterozygous animals obtained were pooled, which subsequently resulted in a mixture of wildtypes, heterozygous and homozygous individuals. Homozygous lines were identified first based on the fluorescent intensity in the eyes after which 10 individuals of each virgin 5 males and 5 females screened at pupal stage were randomly picked and crossed to virgin wildtype of the opposite sex of the same age. The crosses were allowed to stand for 2 weeks for assured mating, after which the individuals are removed, separated and kept in separate vials. Their offsprings were allowed to grow to adults and 
screened for respective fluorescent, and if all the offsprings found in a vial are transgenic (Having the fluorescent) then the founder transgenic parent is considered homozygous and hence kept and crossed with an opposite transgenic sex that its offsprings are all transgenic too.

\section{Molecular characterization of sperm marking lines (Inverse PCR of genomic DNA)}

Genomic DNA sequences flanking 5' and 3' $p B a c$ insertion in the generated sperm marked

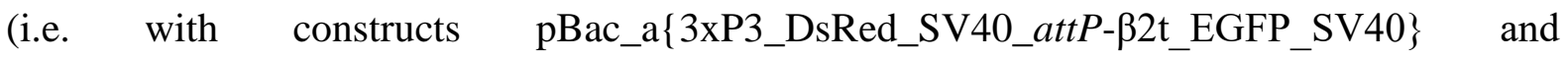

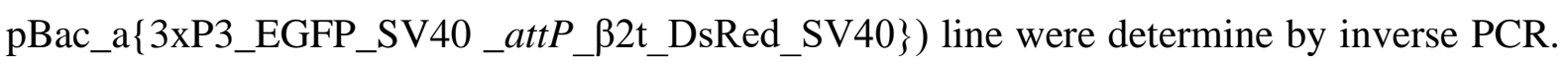
DNA was extracted from the sperm marked individuals using the NucleoSpin ${ }^{\circledR}$ DNA Insect kit (Macherey-Nagel GmbH $\alpha$ Co. KG, Düren, Germany). About $1 \mu \mathrm{g}$ DNA of each transgenic line was digested with $S a u 3 A i$ for 5' arm and MspI for 3' arms for 4 hours and kept at $37^{\circ} \mathrm{C}$ in a $25 \mu 1$ reaction respectively.

The Sau3Ai reactions for 5' arms were inactivated at 65 for 20 minutes. The resulting cut DNA fragments were self-ligated in reaction volume of $400 \mu$ l (i.e. water $333 \mu$, reaction $25 \mu$ l, Buffer $40 \mu \mathrm{l}$ and T4 DNA ligase $2 \mu \mathrm{l}$ ) and kept at $16^{\circ} \mathrm{C}$ overnight. PCRs were conducted on the selfligated genomic DNA from several lines for both 5' and 3' arms using different PCR protocols. For the 5' arm the first PCR was performed using primers iPCR5'F1- 5/ iPCR5'R1-6 (3 min at $98^{\circ} \mathrm{C}, 30 \mathrm{sec}$ at $98^{\circ} \mathrm{C}, 30 \mathrm{sec}$ at $68^{\circ} \mathrm{C}, 1 \mathrm{~min}$ at $72^{\circ} \mathrm{C} 35$ cycles, $10 \mathrm{~min}$ at $72^{\circ} \mathrm{C}$ ). An aliquot of this first reaction served as template for the next nested PCR carried out with primers iPCR5' $\mathrm{F} 2-7 / \mathrm{iPCR}^{\prime} \mathrm{R} 2-8\left(3 \mathrm{~min}\right.$ at $98^{\circ} \mathrm{C}, 30 \mathrm{sec}$ at $98^{\circ} \mathrm{C}, 1: 30 \mathrm{sec}$ at $72^{\circ} \mathrm{C}, 35 \mathrm{cycles}, 10 \mathrm{~min}$ at $\left.72^{\circ} \mathrm{C}\right)$.

For the 3' arm just one PCR reaction was conducted using primers MFS227/MFS228. The products of the PCRs were run on 1\% agarose gel and distinct bands were excised and purified using the NucleoSpin ${ }^{\circledR}$ Gel and PCR Clean-up Kit (Macherey-Nagel GmbH $\alpha$ Co. KG, Düren, Germany).

The purified fragment were cloned into Pjet 1.2 vector ${ }^{40}$ and sequenced using primers Pjet 1.2 forward and reverse primers. Sequences obtained were analyzed in geneious, and Pjet sequences in the result were identified and removed while the gene sequences obtained were used and search for against Tribolium genome Tcas 5.2 using BLAST search to ascertain the localization of the piggybac insertion as shown (Supplementary information 3). Hence the strains F1_M1 and M1_F1 ( pBac_a\{3xP3_EGFP_SV40_attP_B2t_DsRed_SV40\}) and F37_F1 and F37_M2 (pBac_a\{3xP3_DsRed_SV40_attP-32t_EGFP_SV40\}) were used for further studies 


\section{Dissection of Spermatheca, Testis and Single sperms for determination of fluorescence protein expression in the tissues}

Dissection of testis or spermatheca was done by placing adults on ice for 10 minutes. Male or female individual to be dissected is later picked up and placed in a pool of insect saline solution, and by slightly squeezing the thorax of the individual with a pair of forcep the genitalia is exposed, and using another pair of forcep the exposed genitalia is pulled out and moved into ice cold 1xPBS before fixation.

Fixation was done following the Dissecting Tribolium Genitalia protocol (http://coleoguy.blogspot.com/2011/10/dissecting-tribolium-genitalia.html). To obtain the single sperms, the dissected testis was put in $1.5 \mu 1$ Eppendorf tubes and grinded severally using laboratory pestle, and vortexed in between the grinding to facilitate single sperms going into solution. Afterwards the dissected spermatheca and/or testis were placed on a slide singly and further separated carefully before mounting them for microscopy. While the suspended sperms in a solution were pipetted on a slide and covered with a slide before mounting on a microscope.

\section{Spermatheca dissection of a once and twice mated wildtype female}

Two days singly, and 4 and 10 days wildtype spermatheca of twice mated females with EGFP and/or DsRed transgenically sperm marked male individuals were dissected and embedded in OCT embedding matrix (Carl Roth GmbH + Co. KG, Schoemperlen straße 3-5, 76185 Karlsruhe) according to manufacturer instruction and kept in $80^{\circ} \mathrm{C}$ freezer. The spermatheca was cryosection in a cryostat (Leica CM1950, Leica Biosystems Nussloch GmbH Heidelberger Str. 17 - 19 D-69226 Nussloch Germany) machine set at 8mm. The dissected spermatheca were visualized under a fluorescent microscope to determine the dynamic and distribution of the sperms within the reproductive system (spermatheca).

Evaluation of pBac a_3xP3_EGFP-SV40 \{attP-B2t_DsRed_SV40\} and pBac a_3xP3DsRed-SV40 \{attP-B2t_EGFP_SV40\} generated lines

\section{I) Sequential sperm competition assay:}

To determine the sperm competition and utilization by female T. castaneum in a twice mated experiment, we designed a laboratory assay were different marked sperm with either DsRed or EGFP observable (fluorescent) fast folding protein individuals were singly and twice mated with wildtype vermillion ${ }^{\text {white }}\left(v^{w}\right)$ Tribolium females sequentially. A female wildtype Vermillion white $T$. castaneum was either first singly mated with a male expressing either red 
fluorescent sperms pBac a_3xP3_EGFP-SV40\{attP-32t_DsRed_SV40\} (Fig AIV) or male expressing green fluorescent sperms pBac a_3xP3-DsRed-SV40 \{attP-ß2t_EGFP_SV40\} (Fig BIV) for 2 days, after which the male is removed and a corresponding new male with different marked sperm was inoculated with the female and kept for additional 2 days before the male is removed and the female is placed on a new flour. The female is then moved to new flour after every 2 day for a period of 2 months (Supplementary: 1 and 6). Progenies of the assay were screened for eye transformation marker to ascertain the sperm competitiveness of the individuals to sire, and the number obtained were counted and recorded. The experiment was replicated 10 times.

\section{II) Out-crossing and repetition of sequential sperm competition assay to determine any bottleneck effect:}

Homozygous individuals of M1_F1, F1_M1 (pBac \{a_3xP3_EGFP-SV40_attPB2t_DsRed_SV40 $)$ and F37_F1, F37_M2 (pBac \{a_3xP3-DsRed-SV40_attPB2t_EGFP_SV40\}) were singly outcrossed to wildtype $v^{w}$ of the corresponding sex and placed on a $50 \mathrm{~g}$ full grain flour with 12 replications each. The set up were left to stand for a period of 10 days to mate and lay embryos, then the adults were removed and discarded while the embryos laid were left to developed into adults which are heterozygous.

From the 12 replications, i took 3 males from 6 replications and out of the other 6 replications i took 3 females each. 3 males and 3 females were crossed to each other to set up 6 replications, they were kept for 10 days to mate and lay eggs. The adults were subsequently taken out, and the embryos left to develop into adults comprising of wildtype, heterozygous and homozygous individuals. The homozygous individuals were screened based on the high intensity of the fluorescent in the eyes. Subsequently, three homozygous males or females each were screen at pupa stage from the 6 vials, to give 3 sets of males or females individuals, which were then crossed to give a total of 3 crosses at the end. They were left to mate and lay eggs, and after 10 days the adults were removed and kept singly. The offspring were left until they were adults and screened, and when all the offspring sire by mated parent are transgenic, then we know that the parents are homozygous. Thus, the male parents were used to redo the sequential sperm competition assay (Fig: 3 and Supplementary: 6) and other experiments. 


\section{III) Competition assay between different transgenic lines:}

To evaluate the paternity of offsprings sired by different tribolium male individuals in a laboratory test experiments, we used M1_F1, F1_M1 (pBac \{a_3xP3_EGFP-SV40_attPB2t_DsRed_SV40\}) and F37_F1, F37_M2 (pBac \{a_3xP3-DsRed-SV40_attPß2t_EGFP_SV40\}) transgenically marked sperms individuals and mated them with wildtype tribolium $v^{w}$ females. Adult male transgenic strains separated at pupal stage were mated with female wildtype of the same age. The set up was made using 5 transgenic males' strains each expressing different marked sperm, making a total of 10 individuals in each set, and which is eventually crossed with 10 female wildtype individuals in a vail. Each experiment is replicated 3 times. The experiment was allowed to stand initially for a period of 4 days, after which the adults were sieved, and the females were transferred to a new flour and kept for a further 21 days (Supplementary: 2) while the males were discarded. After 21 days the adult females were then removed and discarded, and the offsprings of both sired after 4 day and 21 day were screened by epifluorescence using the eye marker discriminate between the strains and recorded. The experiment was replicated 3 times.

\section{IV) Competition assay of transgenic generated individuals against wildtype.}

To assess the mating/fitness ability of homozygous transgenically marked sperm strains transformed with either DsRed or EGFP constructs against any form of fitness problems due to the introduced foreign constructs in their genome as opposed to their wildtype males counterparts. Also, to test their ability to compete favourably with the wildtype individuals in a preliminary laboratory situation (Supplementary: 3), we set up 3 different experimental assay of a) $1 \times 1 \times 1$ (i.e. normal competition of 5 transgenic males: 5 wildtype males: 5 wildtype females), b) 1x1x2 (i.e. relax competition of 5 transgenic males: 5 wildtype males: 10 wildtype females) and c) $2 \times 2 \times 1$ (i.e. high competition of 10 transgenic males: 10 wildtype males: 5 wildtype females) (Figure 5). All experiments were replicated 3 times and left to stand for 4 days before the males were removed and the female transferred to new flour after every 2 day over a period of 2 months. 


\section{Microscopy/Screening:}

First filial generation of adult beetles were screened for possible transgenic beetles with expected EGFP or DsRed expression in the eyes using LEICA M205 FA stereomicroscope epifluorescence microscope with the filter EGFP-LP (excitation: ET480/40, emission: ET510 LP) and RFP (excitation: ET546/10x, emission: ET605/70m) respectively.

Whole adults, testis, spermatheca and single sperms imaging was done using Zeiss Imager. Z2 equipped with 2 cameras, Axiocam 305 colour for EGFP-LP (excitation: ET480/40, emission: ET510 LP), DsRed (excitation: 533-558, emission: 570-640) or DAPI (excitation: 335-383, emission: 420-470) for cell nuclei staining observation and Axiocam 506 mono for brightfield. The testis or spermatheca were dissected as described by http://coleoguy.blogspot.com/2011/10/dissecting-tribolium-genitalia.html with some modification whereby the tissue were made to stand for 30 minutes using DAPI (4',6Diamidino-2-Phenylindole, Dihydrochloride) for nuclei staining. Afterwards, they were mounted in $70 \%$ glycerol for image capturing. 


\section{Discussion and Conclusion:}

The monitoring and knowledge of biological usage of spermatozoa by inseminated females is a very intensive venture, and also an important aspect of pest management practices for polyandrous insect organism. The investigation of the biological usage of sperms by mated females, mating analysis of multiple mated females as regards to competition for sperms for siring offsprings, as well as monitoring as a component of the AW-SIT ${ }^{27}$ in coleopteran insect pests is important. This, however, can be efficiently achieved using the spermatogenesis specific Tcß2t gene upstream region to mediate the expression of fluorescent protein in the sperms of engineered males.

The regulatory elements of the spermatogenesis Tcß2t gene ${ }^{30}$ are determined and compared to the other organisms i.e. Drosophila melanogaster ${ }^{32}$, Drosophila suzuki ${ }^{33}$ and we showed that the regulatory elements were not conserved in the Tribolium castaneum genome which could be attributed to the fact that they belong to different orders of ins ects.

Furthermore, as in the case of other dipteran ${ }^{26,27,33}$ insects that have being transgenically sperm marked using biotechnological approach, we also present a sperm marked coleopteran insect pest Tribolium castaneum. We were able to use fast folding protein DsRed and EGFP to generate two different types of strains by piggyBac transformation. This provided us with a tool to separate between male from females individuals because of the fluorescent marked sperms within the male testis ${ }^{27,41}$, and this can also be deployed for male only release programmes. Also, successful mating with wildtype mated females can be easily distinguished because of the transfer of the fluorescent marked sperm as we visualize it in their spermatheca under a fluorescent microscope.

The different strains expressing different sperm fluorescent proteins provided an effective tool for efficient downstream experiments such as competition assays ${ }^{16}$, paternity assays 15,29 and also to answered some important biological functions. We found that the dynamic of long term sperm storage in the spermatheca of mated females is obtained, especially for the first mated male, suggesting that fertilization is mostly influenced by the sperm stored in bursa copulatrix ${ }^{42}$, where the sperm of the second mated male is temporary stored before its subsequent mixture in the spermatheca in the long term as shown in our studies.

Bottleneck effects ${ }^{43,44}$ are some of the factors that affect the fitness of individuals in a transgenically generated strain which hitherto affect their ability to compete favourably with 
conspecific wildtype individuals. We performed an outcrossing experiment and repeat the sequential experiment to deal with any bottleneck effect which is possibly diluted. No significant difference was observed when F37_M1 individuals were used as the second inoculated males, and when F37_M2, and M1_F1 were used as the first inoculated male. Also, percentage of individuals sired by F37_M2 in both relax and high competition where non significantly different from the individuals sired by wildtype individuals thus showing no fitness problem as regards to integration of the constructs in the genome of strains in a laboratory competition assay. Thereby, showing the ability for the strain to compete favourably with the wildtype for monitoring ${ }^{13} 45$ purpose in AW-SIT

The use of homozygous individuals for experiment cannot be underestimated in genetic studies. There are several techniques involve for identifying homozygous individual such as 4647 , as well as the intensity of the fluorescent in the eyes or body of transgenic individuals 48 . Hence, following a define pattern we selected 10 individuals each, which were screened at pupal stage and mated with corresponding wildtype individuals. Thus, in an event were all the individuals sired by the transgenic adult turn out to be transgenic then the individual is identified as homozygous 46 .

Previous studies have shown the paternity capabilities of twice mated females in laboratory studies using transgenically marked sperms ${ }^{27}$ in other order of insects. In this study we also provide more proof of paternity in mated $T$. castaneum wildtype females by individuals that have their sperms marked either with DsRed or EGFP flourescent proteins and the result show no significance difference in M1_F1xF37_F1 combination but in the other combinations the transgenically EGFP sperm marked individuals showed significantly more individuals.

Mating individual male from strains that are having their sperm marked with either DsRed or EGFP sequentially with a wildtype female, the strain F37_M2 showed that irrespective of when the males were inoculated the strains compete favorable with wildtype individuals which is not observed in the other strains. This result seems to agree with previous studies where it show that some individuals that are mated first sired more after the sperms of the second mated male have being utilized by the female individual ${ }^{15,29,49,50}$.

In conclusion, we present a transgenic marking of sperm in $T$. castaneum using flourescent proteins which can be efficiently and effectively used for monitoring in an AW-SIT programme because of the ability of the male to transfer the marked sperms into wildtype individuals. Also, 
the strain F37_M2 can compete favourably with the wildtype male individual as shown by our study. In a like manner, we also showed that the female individuals uses the sperm of the second inseminate male first, before using the inseminated sperms of the first mated male ${ }^{42}$. However, our study also show that the sperm stored by the female can used for a long time in contrast to other reports ${ }^{42}$. Thus, based on that it gives us a tool to further study basic reproductive biology regarding sperm utilization over a long period of time in the polyandrous female. 


\section{REFERENCES}

1. Schmitt-Engel, C. et al. The iBeetle large-scale RNAi screen reveals gene functions for insect development and physiology. Nat Commun 6, 1-10 (2015).

2. S.A. Slipinski et al., 2011. In Z.-Q. Zhang (ed.), Animal biodiversity: An outline of higher-level classification and survey of taxonomic richness. Zootaxa 3148 - Reference Details - The Taxonomicon. http://taxonomicon.taxonomy.nl/Reference.aspx?id=7112.

3. Grove, S. J. \& Stork, N. E. An inordinate fondness for beetles. Invert. Systematics 14, $733-739$ (2000).

4. R.A. Crowson, 1981. The biology of the Coleoptera. https://www.zin.ru/Animalia/Coleoptera/rus/crows981.htm.

5. Mantzoukas, S. et al. Postharvest Treatment of Tribolium confusum Jacquelin du Val Adults with Commercial Biopesticides. Agriculture 9, 226 (2019).

6. Foottit, R. G. \& Adler, P. H. Insect Biodiversity: Science and Society. (John Wiley \& Sons, 2009).

7. Kumar, H. et al. Red flour beetle (Tribolium castaneum): From population genetics to functional genomics. Vet World 11, 1043-1046 (2018).

8. El-Desouky, T. A., Elbadawy, S. S., Hussain, H. B. H. \& Hassan, N. A. Impact of Insect Densities on the Benzoquinone Secretions and Aflatoxins Levels in Wheat Flour During Storage Periods. The Open Biotechnology Journal 12, (2018).

9. Wijayaratne, L. K. W., Fields, P. G. \& Arthur, F. H. Residual Efficacy of Methoprene for Control of Tribolium castaneum (Coleoptera: Tenebrionidae) Larvae at Different Temperatures on Varnished Wood, Concrete, and Wheat. J Econ Entomol 105, 718-725 (2012). 
10. Arthur, F. H. Efficacy of chlorfenapyr against Tribolium castaneum and Tribolium confusum (Coleoptera: Tenebrionidae) adults exposed on concrete, vinyl tile, and plywood surfaces. Journal of Stored Products Research 44, 145-151 (2008).

11. Singh, S. \& Prakash, S. Development of resistance in Tribolium castaneum, Herbst (Coleoptera: Tenebrionidae) towards deltamethrin in laboratory. 3, 4 (2013).

12. Köhl, J., Kolnaar, R. \& Ravensberg, W. J. Mode of Action of Microbial Biological Control Agents Against Plant Diseases: Relevance Beyond Efficacy. Front. Plant Sci. 10, (2019).

13. Campbell, J. F., Mullen, M. A. \& Dowdy, A. K. Monitoring Stored-Product Pests in Food Processing Plants with Pheromone Trapping, Contour Mapping, and MarkRecapture. JOURNAL OF ECONOMIC ENTOMOLOGY 95, 13 (2002).

14. Lewis, S. M. \& Jutkiewicz, E. Sperm Precedence and Sperm Storage in Multiply Mated Red Flour Beetles. Behavioral Ecology and Sociobiology 43, 365-369 (1998).

15. Lewis, S. M., Kobel, A., Fedina, T. \& Beeman, R. W. Sperm stratification and paternity success in red flour beetles. Physiol Entomol 30, 303-307 (2005).

16. Favila, M. E., Nolasco, J., Florescano, I. C. \& Equihua, M. Sperm competition and evidence of sperm fertilization patterns in the carrion ball-roller beetle Canthon cyanellus cyanellus LeConte (Scarabaeidae: Scarabaeinae). Behav Ecol Sociobiol 59, $38-43(2005)$.

17. Roderick, G. K., de Mendoza, L. G., Dively, G. P. \& Follett, P. A. Sperm Precedence in Colorado Potato Beetle, $<$ I $>$ Leptinotarsa decemlineata $</$ I $>$ (Coleoptera: Chrysomelidae): Temporal Variation Assessed by Neutral Markers. an 96, 631-636 (2003).

18. Alphey, L. et al. Genetic technologies to enhance the Sterile Insect Technique (SIT). 8. 
19. Harrell, C. R., Djonov, V., Fellabaum, C. \& Volarevic, V. Risks of Using Sterilization by Gamma Radiation: The Other Side of the Coin. Int J Med Sci 15, 274-279 (2018).

20. Franz, G., Gencheva, E. \& Kerremans, Ph. Improved stability of genetic sex-separation strains for the Mediterranean fruit fly, Ceratitis capitata. Genome 37, 72-82 (1994).

21. Hendrichs, J., Franz, G. \& Rendon, P. Increased effectiveness and applicability of the sterile insect technique through male-only releases for control of Mediterranean fruit flies during fruiting seasons. Journal of Applied Entomology 119, 371-377 (1995).

22. Niyazi, N. et al. Genetics and Mating Competitiveness of Ceratitis capitata (Diptera: Tephritidae) Strains Carrying the Marker Sergeant, Sr2. Ann Entomol Soc Am 98, 119125 (2005).

23. Parker, A. G. Mass-Rearing for Sterile Insect Release. in Sterile Insect Technique: Principles and Practice in Area-Wide Integrated Pest Management (eds. Dyck, V. A., Hendrichs, J. \& Robinson, A. S.) 209-232 (Springer Netherlands, 2005). doi:10.1007/14020-4051-2_8.

24. Hagler, J. R. \& Jackson, C. G. Methods for marking insects: current techniques and future prospects. Annu. Rev. Entomol. 46, 511-543 (2001).

25. Catteruccia, F., Benton, J. P. \& Crisanti, A. An Anopheles transgenic sexing strain for vector control. Nature Biotechnology 23, 1414-1417 (2005).

26. Smith, R. C., Walter, M. F., Hice, R. H., O’Brochta, D. A. \& Atkinson, P. W. Testisspecific expression of the beta2 tubulin promoter of Aedes aegypti and its application as a genetic sex-separation marker. Insect Mol. Biol. 16, 61-71 (2007).

27. Scolari, F. et al. Fluorescent sperm marking to improve the fight against the pest insect Ceratitis capitata (Wiedemann; Diptera: Tephritidae). New Biotechnology 25, 76-84 (2008). 
28. Zimowska, G. J., Nirmala, X. \& Handler, A. M. The beta2-tubulin gene from three tephritid fruit fly species and use of its promoter for sperm marking. Insect Biochem. Mol. Biol. 39, 508-515 (2009).

29. Scolari, F. et al. Polyandry in the medfly - shifts in paternity mediated by sperm stratification and mixing. BMC Genet 15, S10 (2014).

30. Siebert, K. S., Lorenzen, M. D., Brown, S. J., Park, Y. \& Beeman, R. W. Tubulin superfamily genes in Tribolium castaneum and the use of a Tubulin promoter to drive transgene expression. Insect Biochemistry and Molecular Biology 38, 749-755 (2008).

31. iBeetle. http://ibeetle-base.uni-goettingen.de/.

32. Michiels, F., Gasch, A., Kaltschmidt, B. \& Renkawitz-Pohl, R. A 14 bp promoter element directs the testis specificity of the Drosophila beta 2 tubulin gene. EMBO J 8, 1559-1565 (1989).

33. Ahmed, H. M. M., Hildebrand, L. \& Wimmer, E. A. Improvement and use of CRISPR/Cas9 to engineer a sperm-marking strain for the invasive fruit pest Drosophila suzukii. BMC Biotechnol 19, 85 (2019).

34. Eckermann, K. N. et al. Hyperactive piggyBac transposase improves transformation efficiency in diverse insect species. Insect Biochemistry and Molecular Biology 98, 1624 (2018).

35. Berghammer, A. J., Klingler, M. \& A. Wimmer, E. A universal marker for transgenic insects. Nature 402, 370-371 (1999).

36. Shagin, D. A. et al. GFP-like proteins as ubiquitous metazoan superfamily: evolution of functional features and structural complexity. Mol. Biol. Evol. 21, 841-850 (2004).

37. Bevis, B. J. \& Glick, B. S. Rapidly maturing variants of the Discosoma red fluorescent protein (DsRed). Nat. Biotechnol. 20, 83-87 (2002). 
38. Horn, C. \& Wimmer, E. A versatile vector set for animal transgenesis. Development genes and evolution 210, 630-7 (2001).

39. Horn, C., Schmid, B. G. M., Pogoda, F. S. \& Wimmer, E. A. Fluorescent transformation markers for insect transgenesis. Insect Biochem. Mol. Biol. 32, 1221-1235 (2002).

40. CloneJET ${ }^{\mathrm{TM}}$ PCR-Klonierungskit. https://www.thermofisher.com/order/catalog/product/K1231.

41. Krzywinska, E. \& Krzywinski, J. Analysis of expression in the Anopheles gambiae developing testes reveals rapidly evolving lineage-specific genes in mosquitoes. $B M C$ Genomics 10, 300 (2009).

42. Droge-Young, E. M., Belote, J. M., Perez, G. S. \& Pitnick, S. Resolving mechanisms of short-term competitive fertilization success in the red flour beetle. Journal of Insect Physiology 93-94, 1-10 (2016).

43. Schetelig, M. F. \& Wimmer, E. A. Insect Transgenesis and the Sterile Insect Technique. in Insect Biotechnology (ed. Vilcinskas, A.) 169-194 (Springer Netherlands, 2011). doi:10.1007/978-90-481-9641-8_9.

44. Wimmer, E. A. Applications of insect transgenesis. Nat Rev Genet 4, 225-232 (2003).

45. Visarada, K., Cr, A., Srujana, S., Nammi, S. \& Seetharama, N. Transgenic Breeding: Perspectives and Prospects. Crop Science 49, (2009).

46. Strobl, F., Ross, J. A. \& Stelzer, E. H. K. Non-lethal genotyping of Tribolium castaneum adults using genomic DNA extracted from wing tissue. PLoS One 12, (2017).

47. Strobl, F., Anderl, A. \& Stelzer, E. H. A universal vector concept for a direct genotyping of transgenic organisms and a systematic creation of homozygous lines. eLife 7,. 
48. Gratz, S. J. et al. Highly specific and efficient CRISPR/Cas9-catalyzed homologydirected repair in Drosophila. Genetics 196, 961-971 (2014).

49. Bernasconi, G. \& Keller, L. Female polyandry affects their sons' reproductive success in the red flour beetle Tribolium castaneum: Female polyandry and sons' reproductive success. Journal of Evolutionary Biology 14, 186-193 (2001).

50. Pai, A. \& Yan, G. Female mate choice in relation to heterozygosity in Tribolium castaneum: Female mate choice and heterozygosity. Journal of Evolutionary Biology 15, 1076-1082 (2002). 


\section{FIGURES:}

A) Wildtype
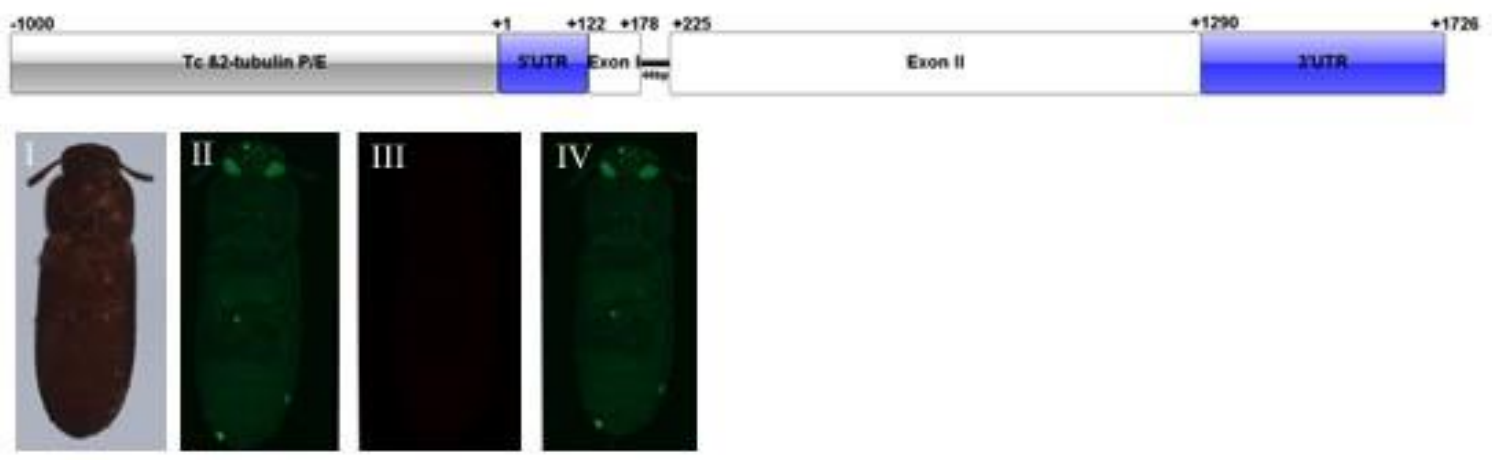

B) $\mathrm{p} B a c\left\{a \_3 x P 3 \_E G F P-S V 40 \_\right.$attP-B2t_DsRed_SV 40\}

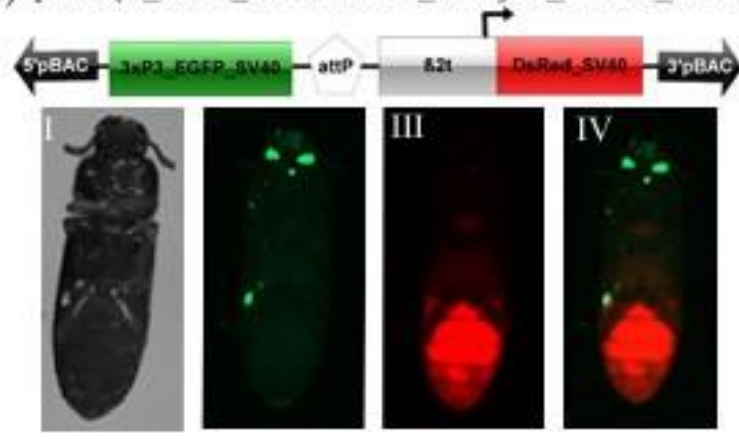

C) pBac $\left\{a \_3 x P 3-D s R e d-S V 40 \_\right.$attP-B2t_EGFP_SV40\}

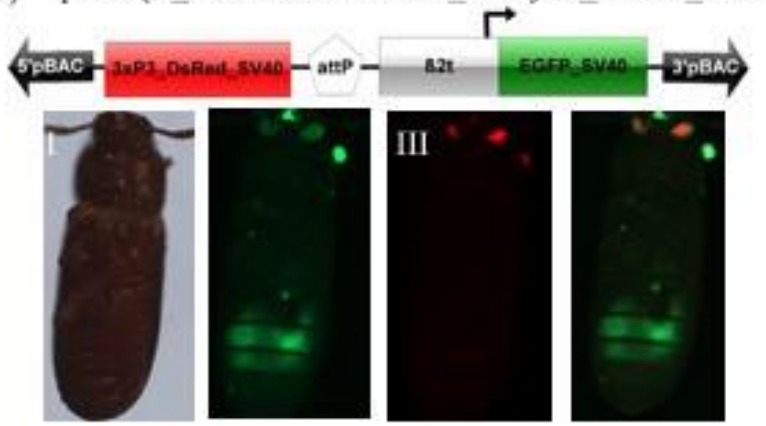

FIGURE: 1. Result showing A) Schematic depiction of endogenous TC009035 gene showing the EXON1 and 2 in white, Intron in black and 5'UTR and 3'UTR in grey adult and wildtype male individual, (B) and (C) constructs and males transgenic individuals: Ventral images of adult males (BI-IV) and (CI-IV) transformed by (B) and (C) constructs respectively. The images were taken with GFP-LP and/or RFP and brightfield filters. Compared to wild type (AII and AIII), the eyes and testes of the transgenic individuals show strong expression of EGFP (BII) and DsRed (CIII) under control of the 3xP3 promoter in the eyes, and DsRed (BIII) and EGFP (CII) in the testis. In contrast to wild type females mated to wild type males showed no DsRed or EGFP fluorescent protein were detected in the eyes or sperm of the individuals. 
A)
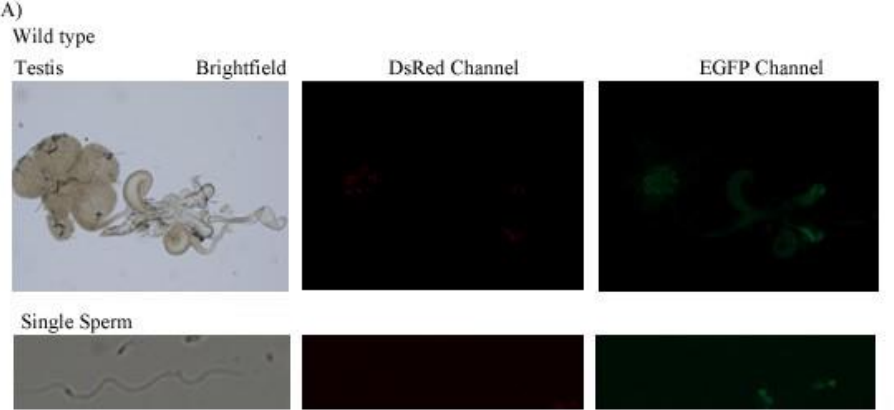

Spermatheca
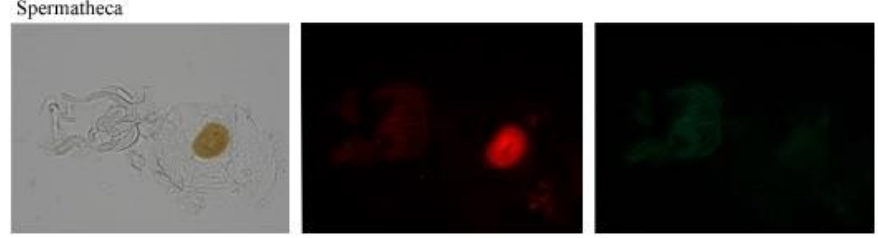

B)

pBac \{a_3xP3 EGFP_SV 40_attP- $\beta 2 t$ DsRed SV 40\}

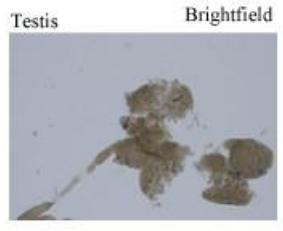

DsRed Channel

EGFP Channel
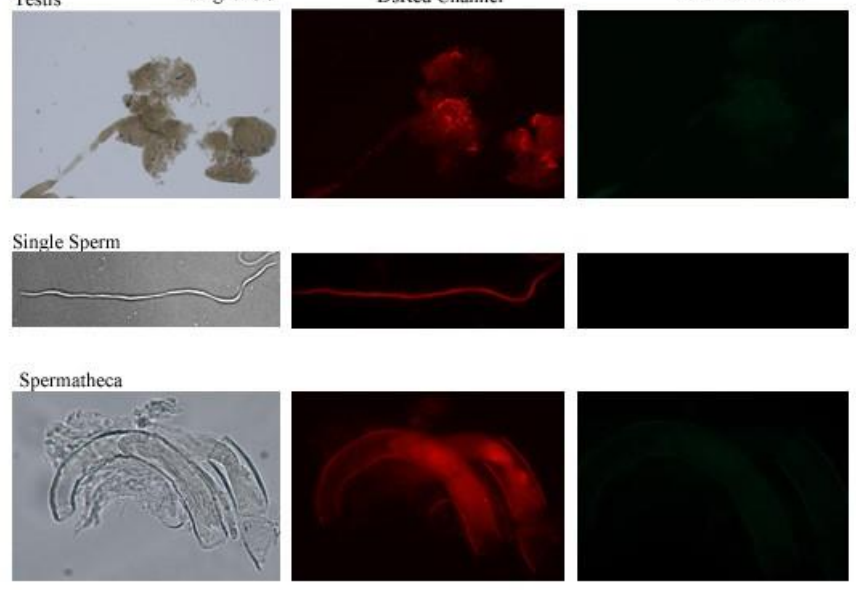

C)

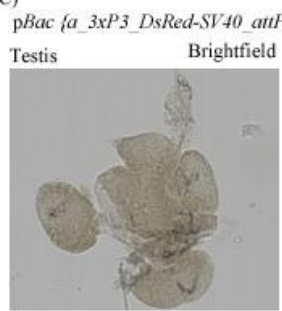

Brightfield

DsRed Channel

EGFP Channel
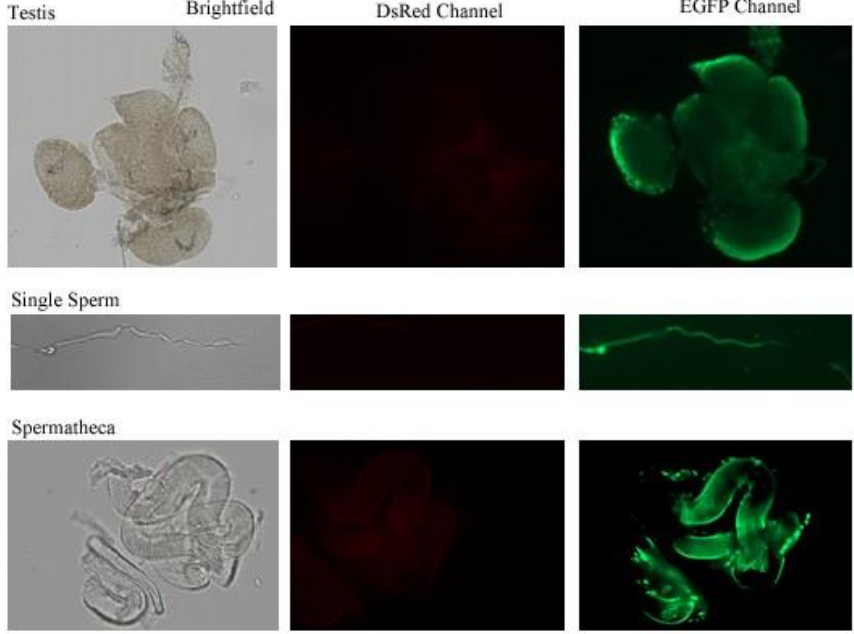

FIGURE 2: Result show the possibility of fluorescence expression of DsRed and EGFP fluorescence protein in wildtype (WT) and transgenic individuals' testes, spermathecae of WT female mated with either transgenic or wildtype males and single sperm extracted for WT or transgenic individuals. (A) Weak green autofluorescence in the testis of the WT ales but no red fluorescence was observed. No fluorescence in the WT single sperm was observed, however show weak red autofluorescence was observed in the WT female spermatheca. (B)The testes and single sperm from transgenic males and the spermatheca of WT female mated to a male of pBac_a $\left\{3 x P 3 \_E G F P \_S V 40 \_a t t P \_\beta 2 t \_D s R e d \_S V 40\right\}$ strains show strong red fluorescence only. (C)The testes and the single sperm from transgenic males and the spermatheca of WT female mated to a male of pBac_a $\left\{3 \times P 3\right.$ _DsRed_SV40_attP- $\left.\beta 2 t \_E G F P \_S V 40\right\}$ strains show green fluorescence. 
F1_M1 x F37_F1

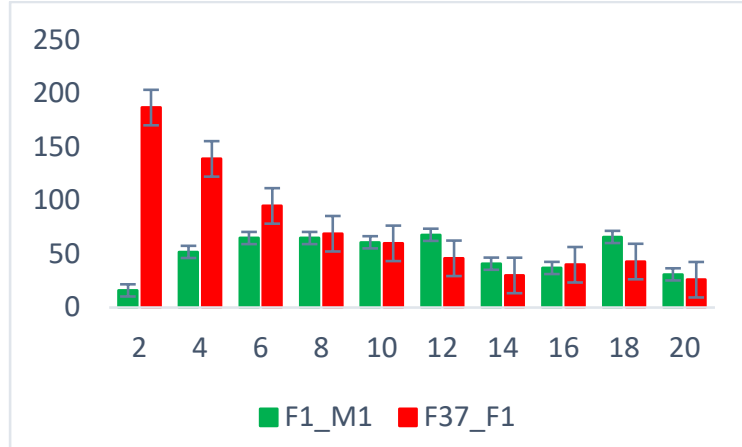

F1_M1 x F37_M2

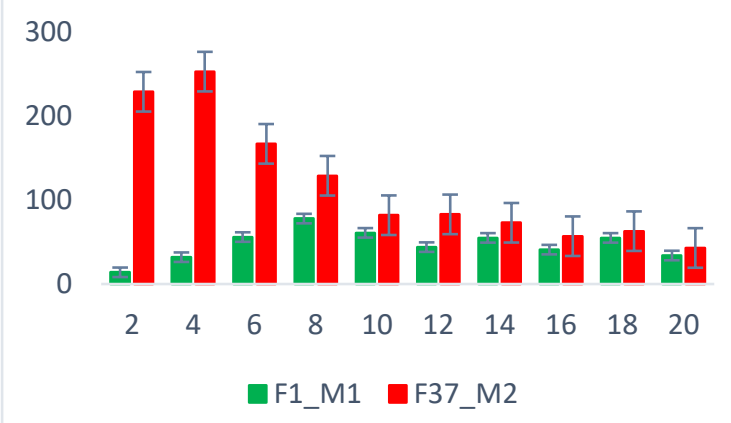

M1_F1 x F37_F1

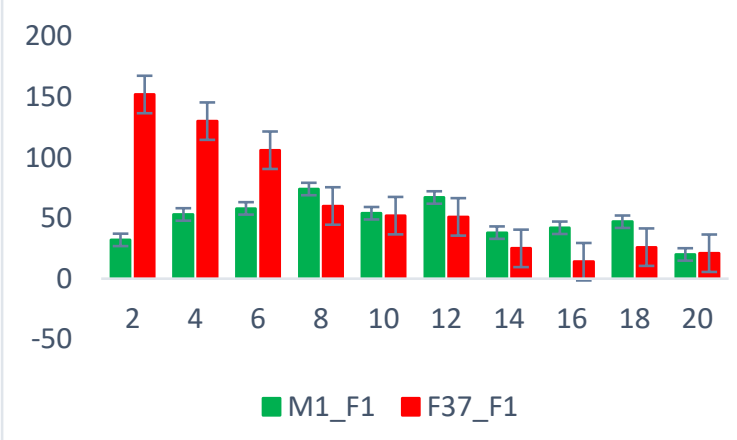

M1_F1 x F37_M2

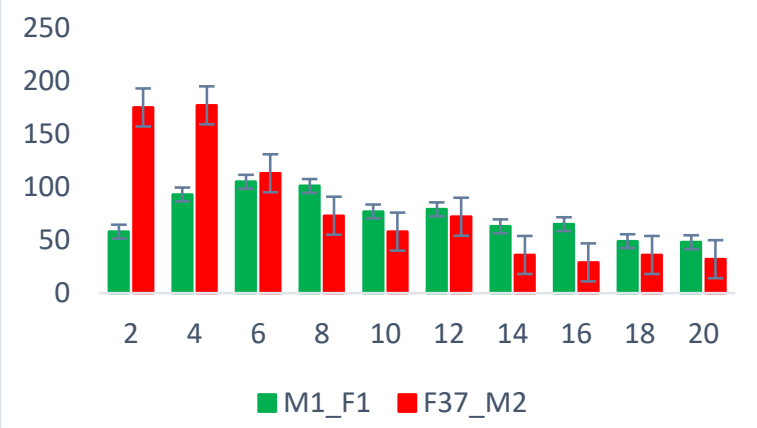

F37_F1 x F1_M1

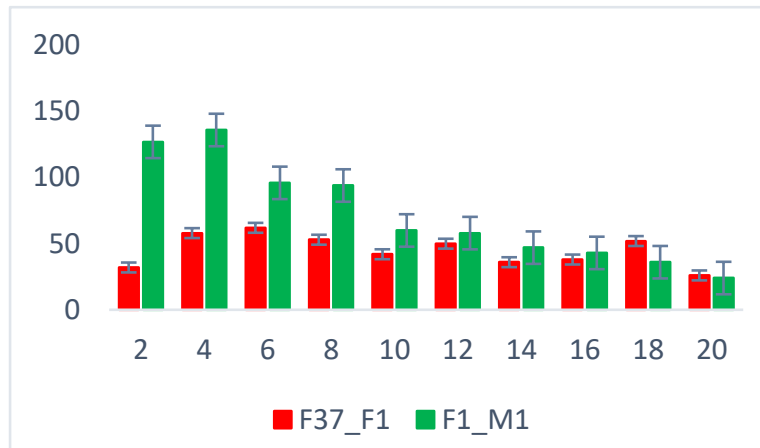

F37_M2 x F1_M1

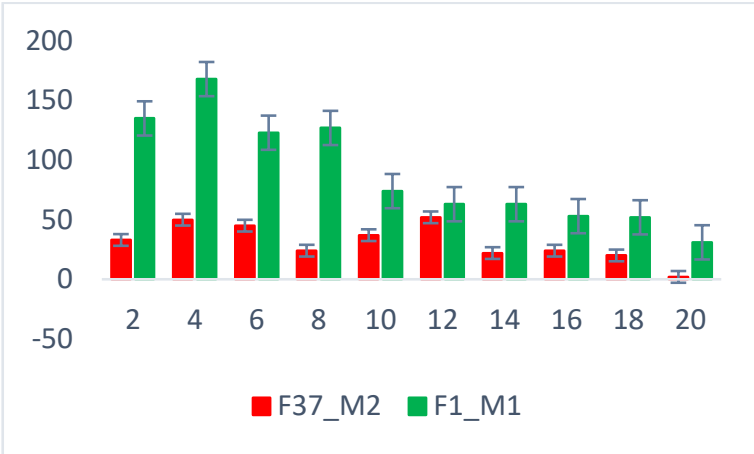

F37_F1 x M1_F1

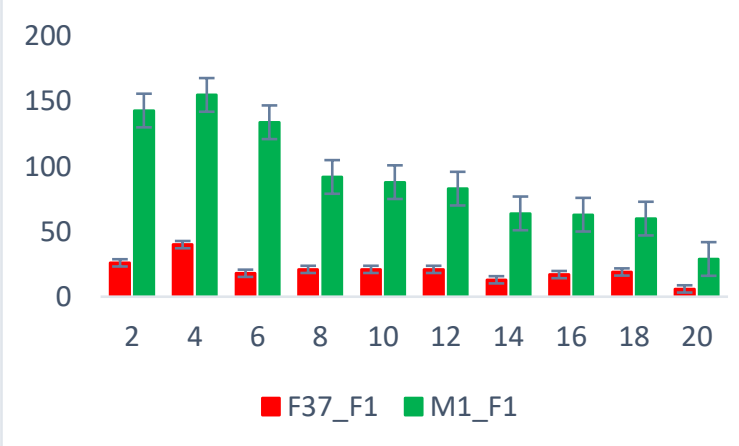

F37_M2 x M1_F1

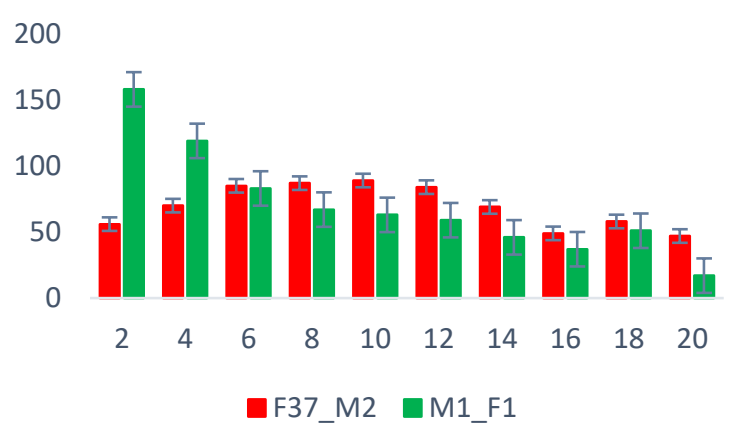

FIGURE 3: Out-crossing and repetition of sperm competition assay 


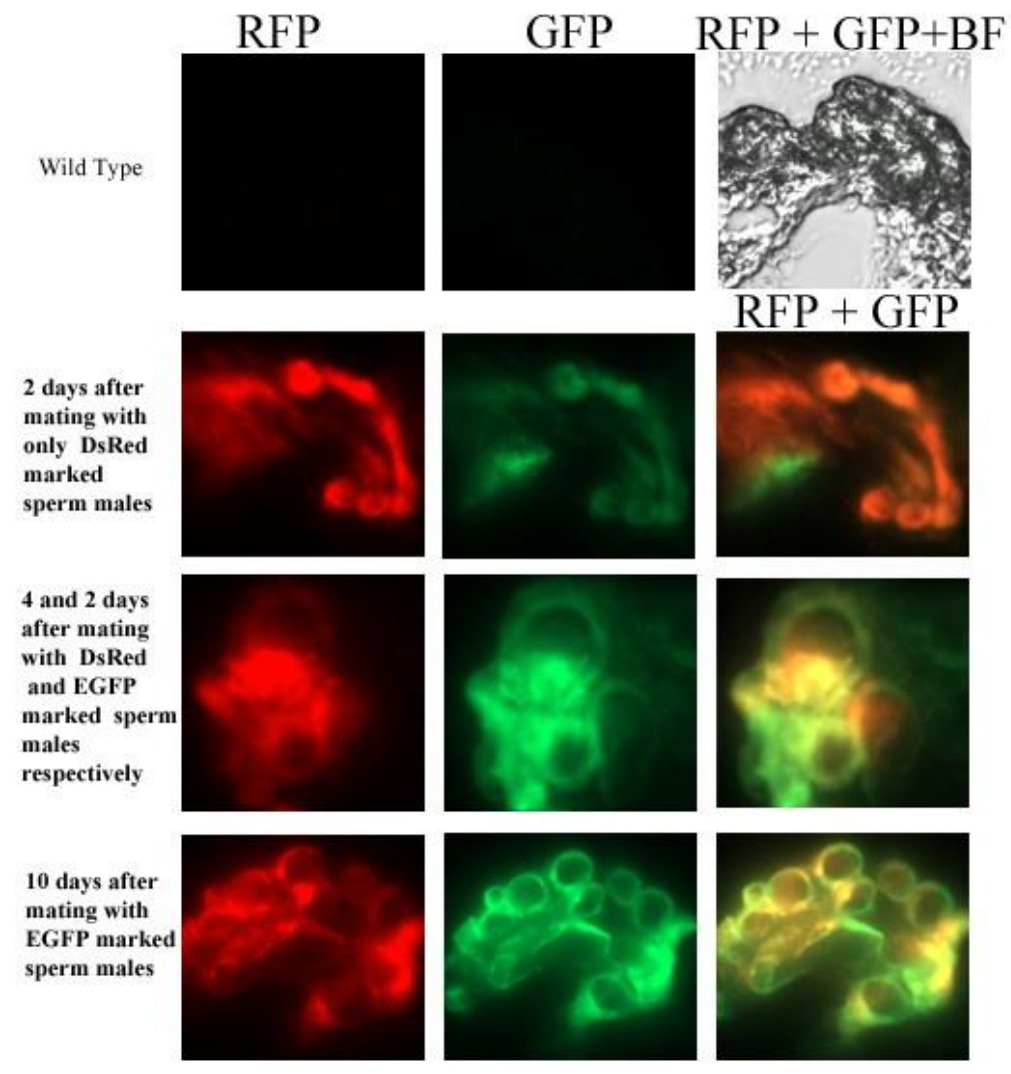

FIGURE 4a: Result showing a cryosection spermatheca of WT female mated with WT males, and of once or twice WT females mated with either red marked sperm males only and/or red marked sperm males first followed by green marked sperm males and sperm distribution visualized over number of days

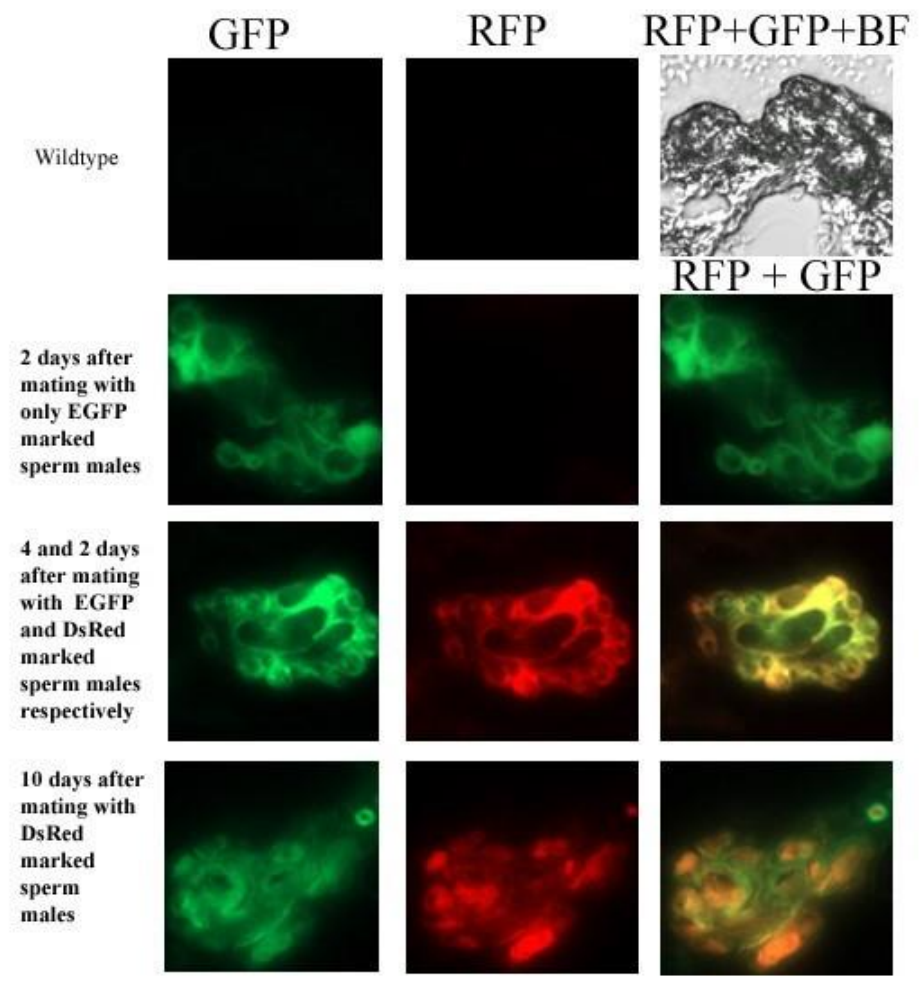

FIGURE 4b: Result showing a cryosection spermatheca of WT female mated with WT males, and of once or twice WT females mated with either green marked sperm males only and/or green marked sperm males first followed by red marked sperm males and sperm distribution visualized over number of days 


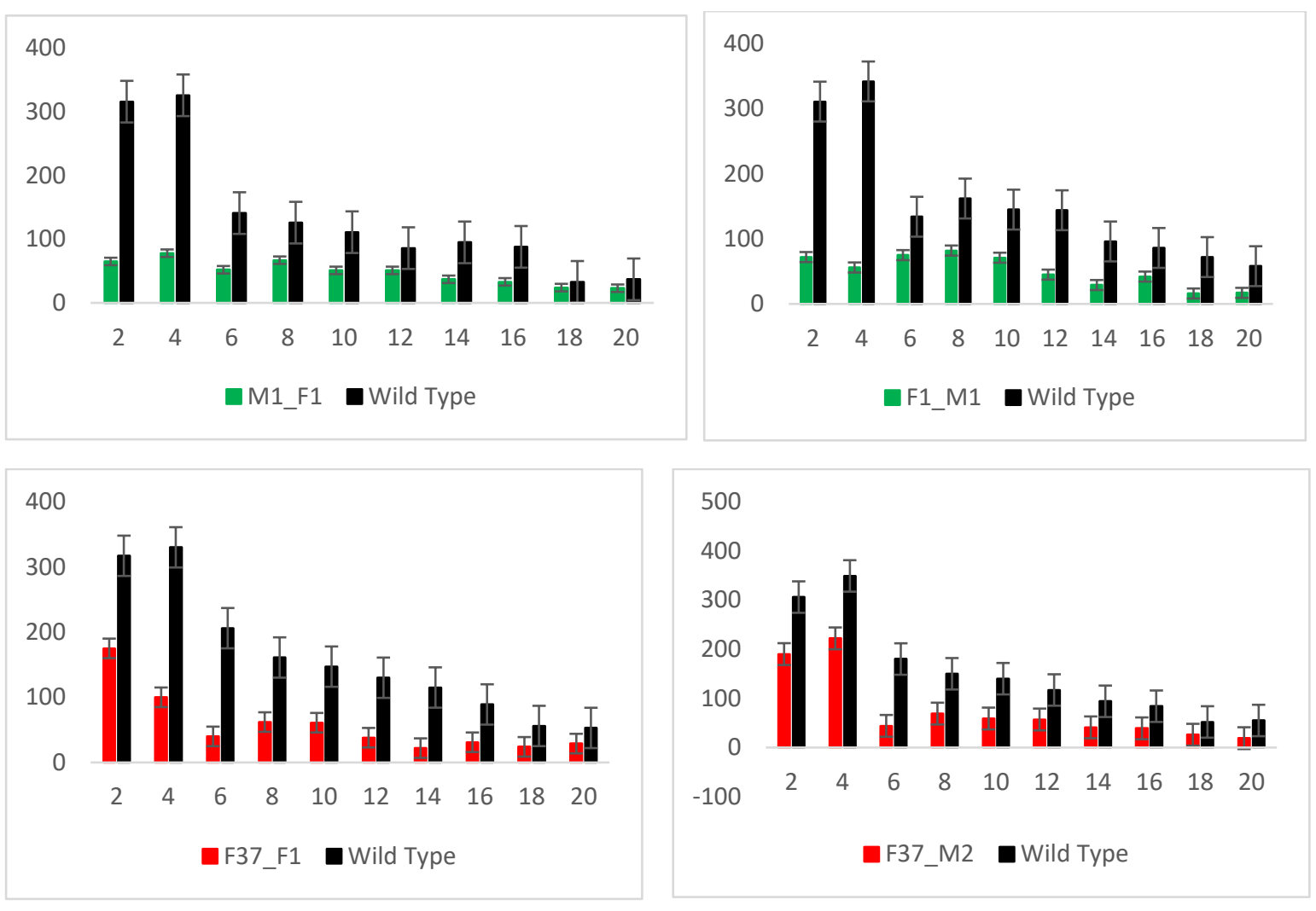

FIGURE 5a: 5 Transgenic males x 5 Wild type male $x$ Wild Type female (Normal Competition)

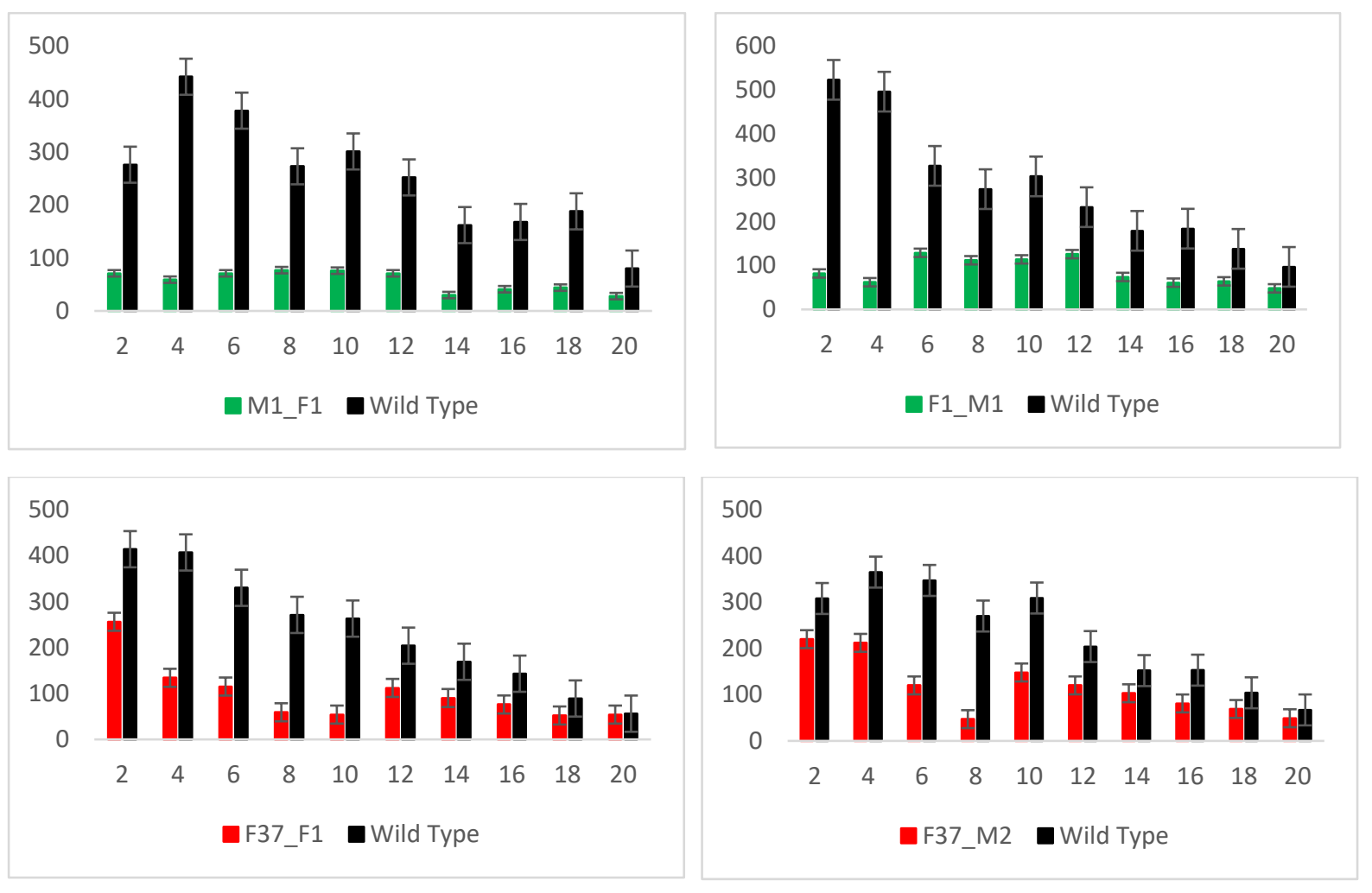

FIGURE 5b: 5 Transgenic males x 5 Wild type male x 10 Wild Type female (Relax Competition) 

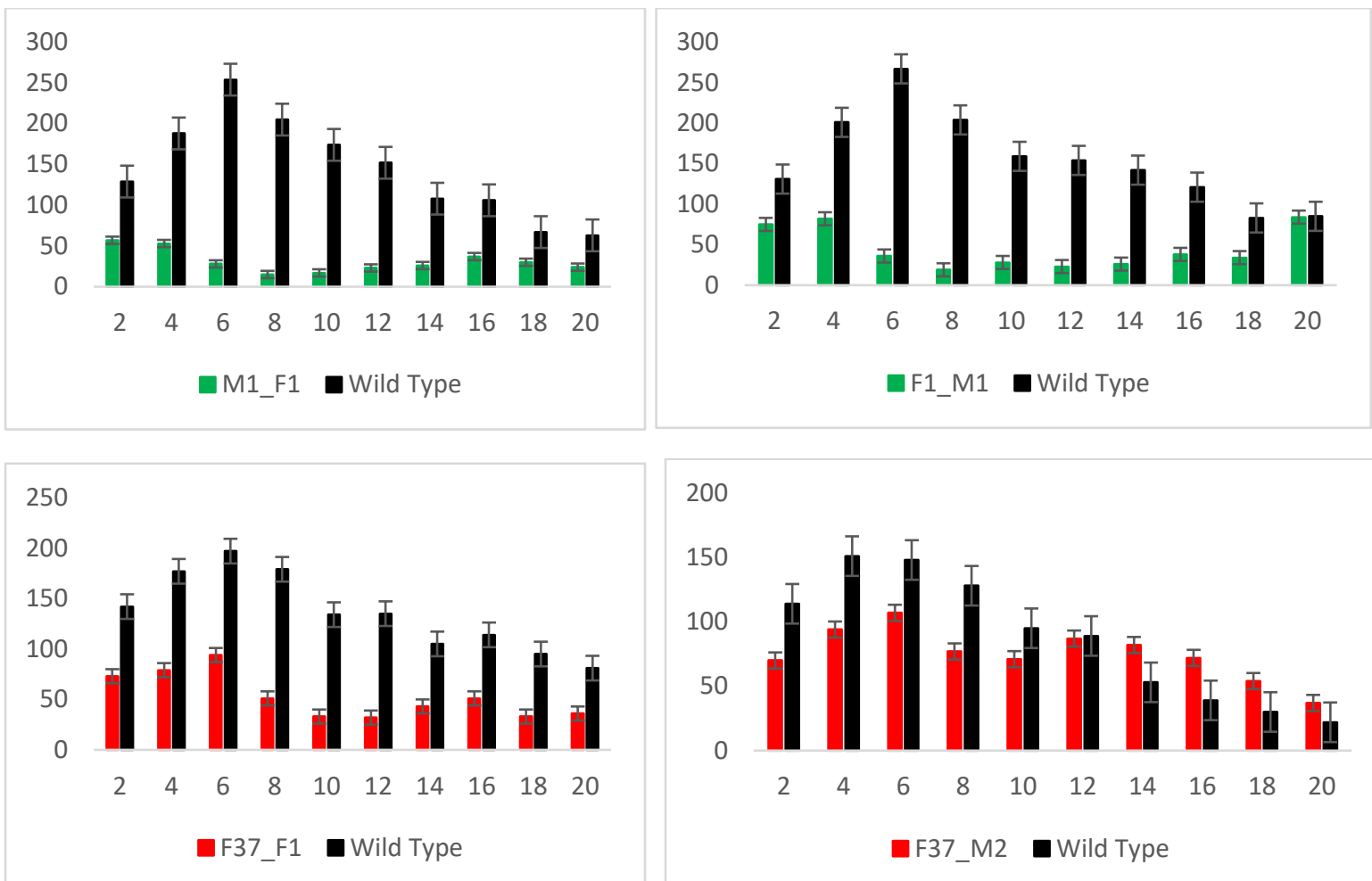

FIGURE 5c: 10 Transgenic males x 10 Wild type male x 5 Wild Type female (High Competition) 


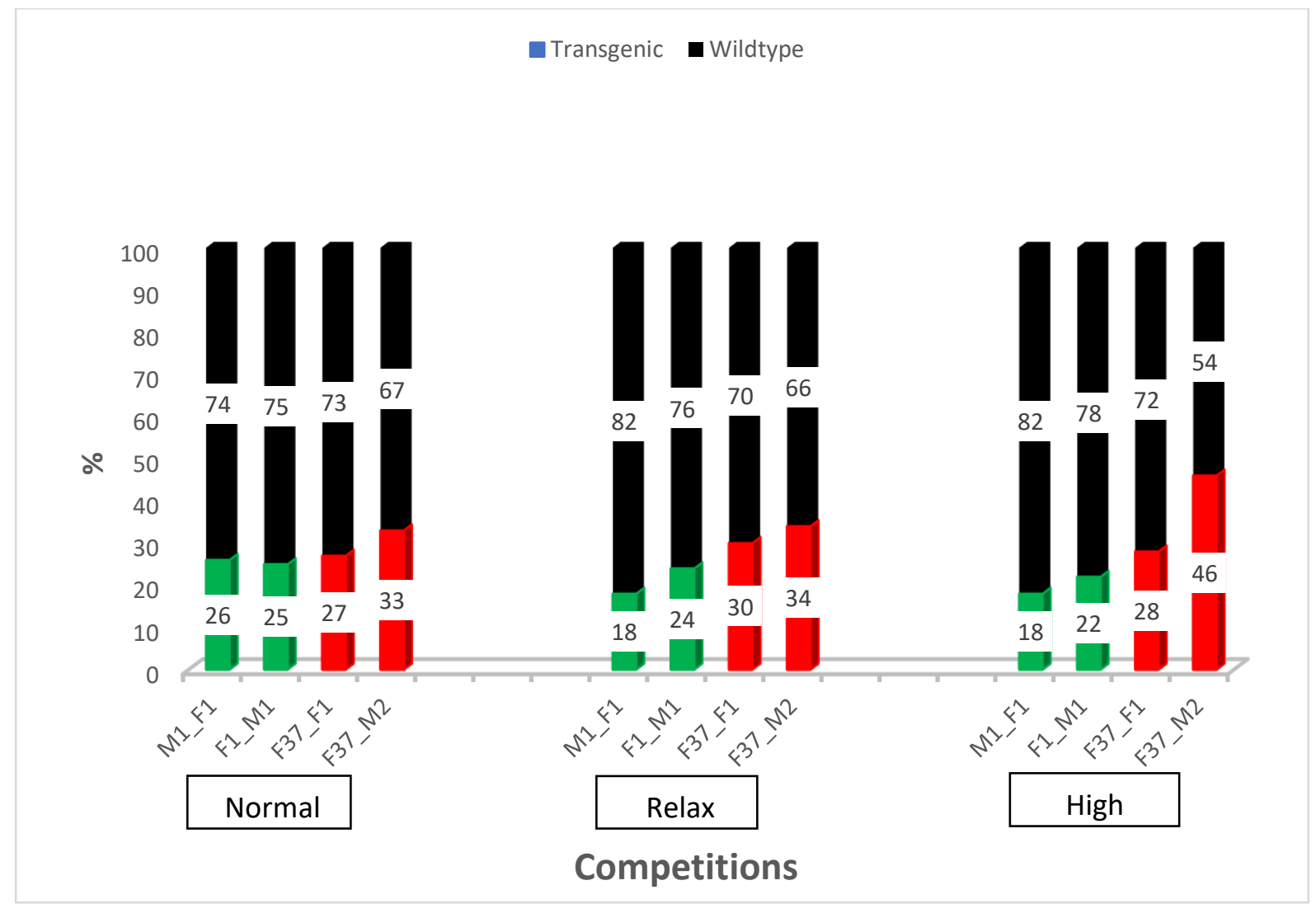

FIGURE 6: Percentage of offsprings sired by transgenic male strains and wildtype male individuals in mating/fitness assay. 
Table 1: LIST OF TRANSGENIC LINES.

\begin{tabular}{|c|c|c|}
\hline CONSTRUCT & $\mathbf{G}_{\mathbf{0}}$ & $\begin{array}{l}\text { TRANSGENIC } \\
\text { LINE }\end{array}$ \\
\hline pBac_\{a_3xP3_DsRed_SV40_attP- $\beta 2 \mathrm{t}$ EGFP_SV40\} & $\begin{array}{l}\text { 1) } \mathrm{M} 1 \\
\text { 2) } \mathrm{M} 2 \\
\text { 3) } \mathrm{F} 1 \\
\text { 4) } \mathrm{F} 11\end{array}$ & $\begin{array}{l}\text { M1_M1, M1_M2, } \\
\text { M1_F1, M1_F2. } \\
\text { M2_M4, M2_F1, } \\
\text { M2_F3 } \\
\text { F1_M1, F1_F4, } \\
\text { F1_F5, M4_M1, } \\
\text { M4_F1, M4_M3 } \\
\text { F11_F1, F11_F2, } \\
\text { F11_M1, M5_F1, } \\
\text { M5_M1 }\end{array}$ \\
\hline pBac_\{a_3xP3_EGFP_SV40_attP_B2t_DsRed_SV40\} & $\begin{array}{ll}\text { 1) } & \text { F37 } \\
\text { 2) } & \text { M17 } \\
\text { 3) } & \text { M7 }\end{array}$ & $\begin{array}{l}\text { F37_F1 and } \\
\text { F37_M2 } \\
\text { M17_M1 } \\
\text { M7_M1 }\end{array}$ \\
\hline
\end{tabular}

Table 2: Transformation efficiency of sperm marking constructs via piggyBac germline mediated germline transformation 


\section{SUPPLEMENTARY FIGURES}

\section{F1_M1 x F37_F1}

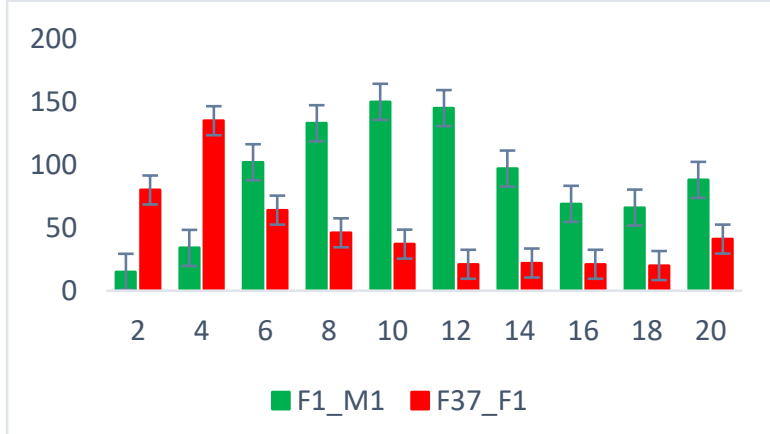

F1_M1 x F37_F2

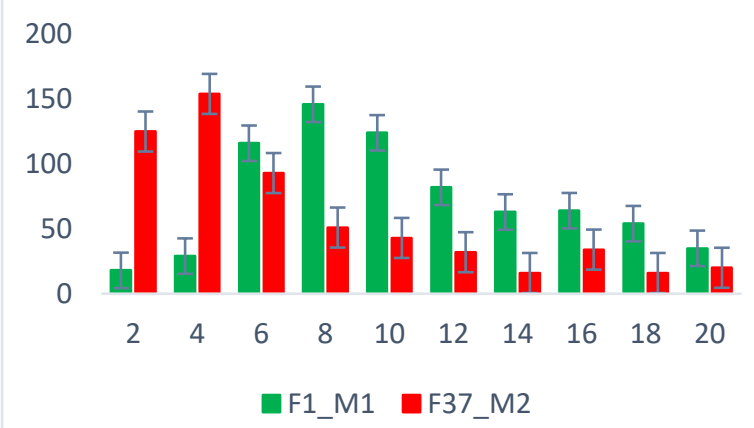

\section{M1_F1 x F37_F1}

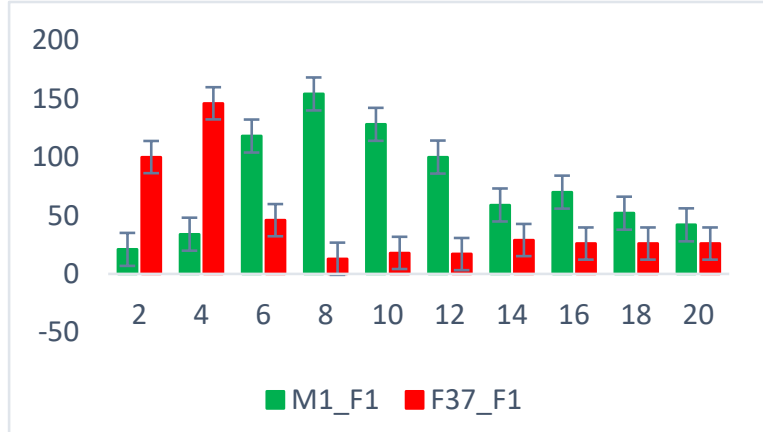

\section{M1_F1 x F37_F2}

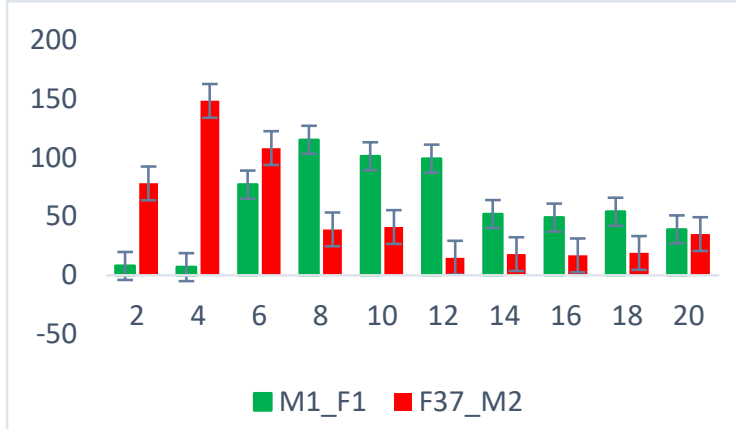

\section{F37_F1 x F1_M1}

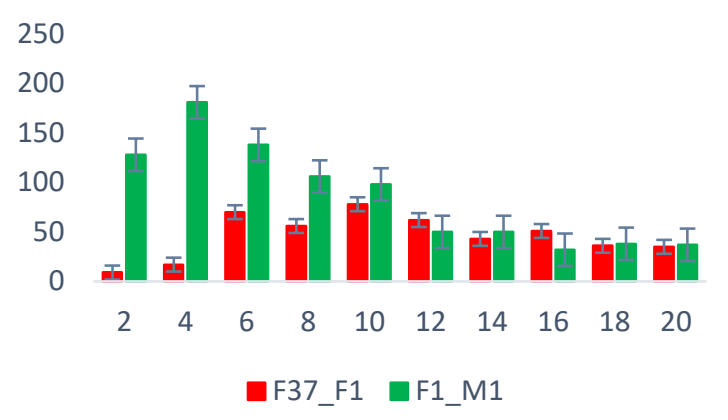

F37_F2 x F1_M1

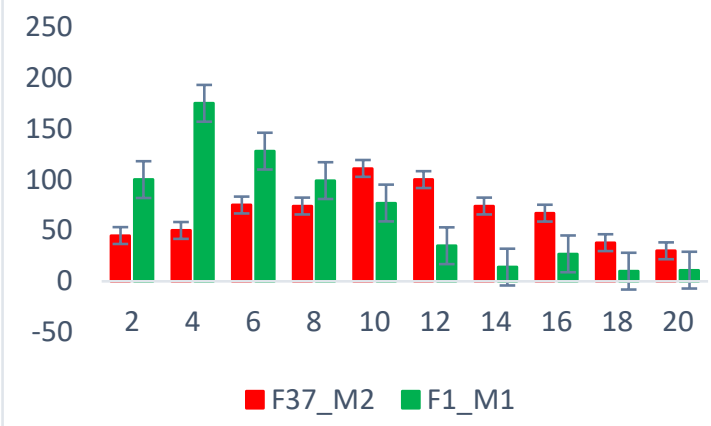

\section{F37_F1 x M1_F1}

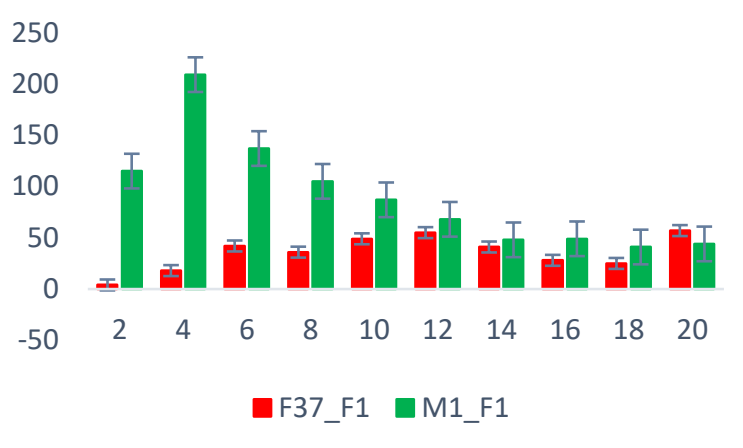

F37_F2 x M1_F1

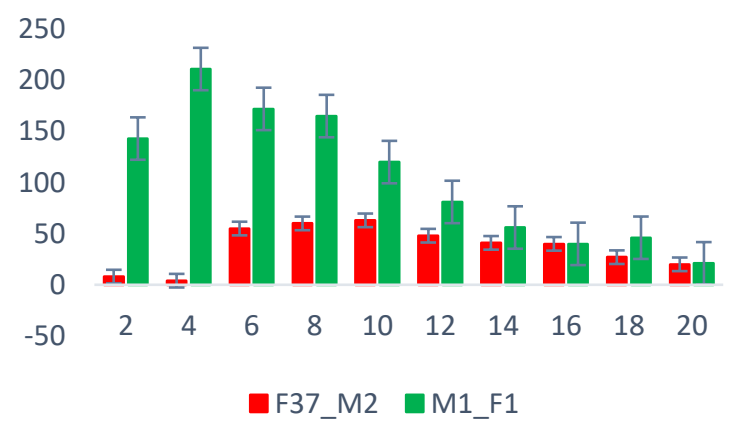

Supplementary Fig 1: sperm competition assay before Out-crossing. 
A)

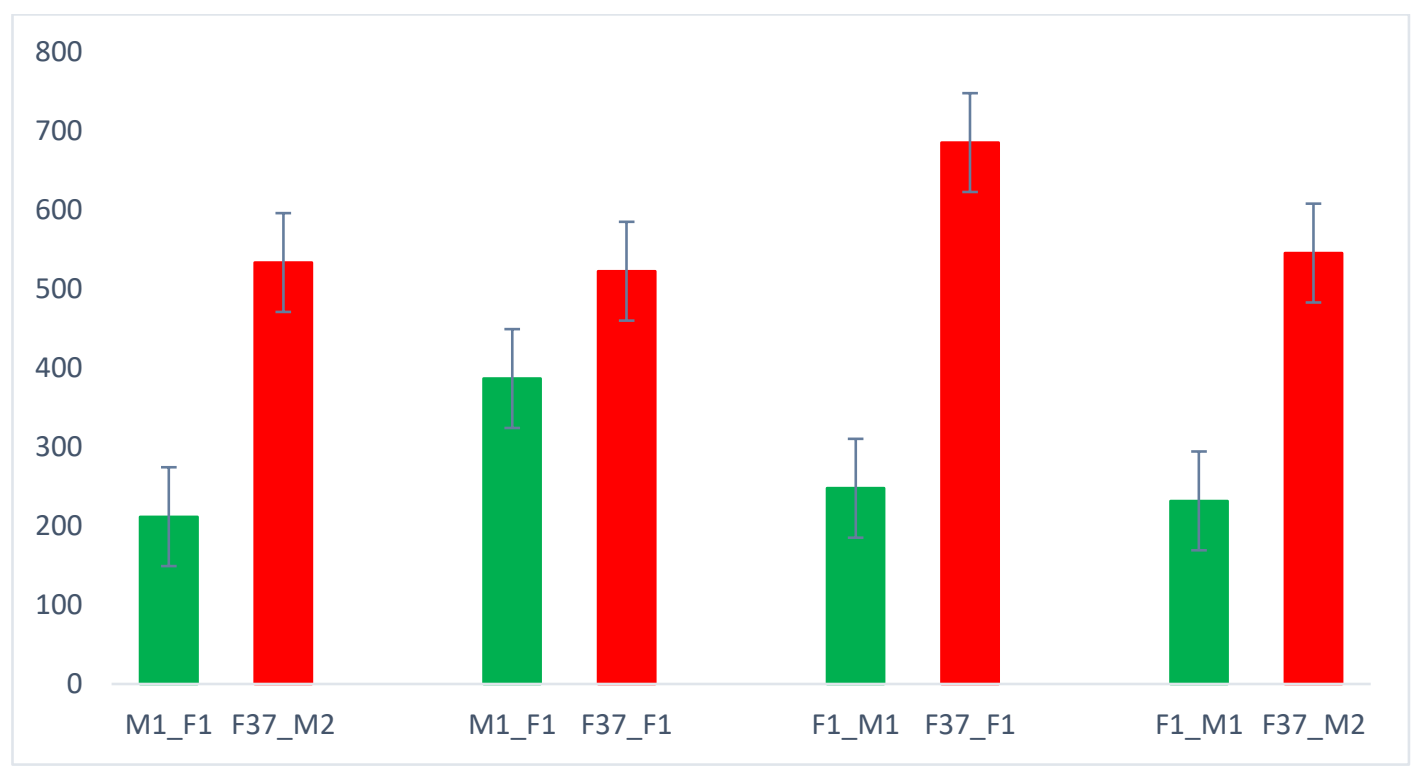

B)

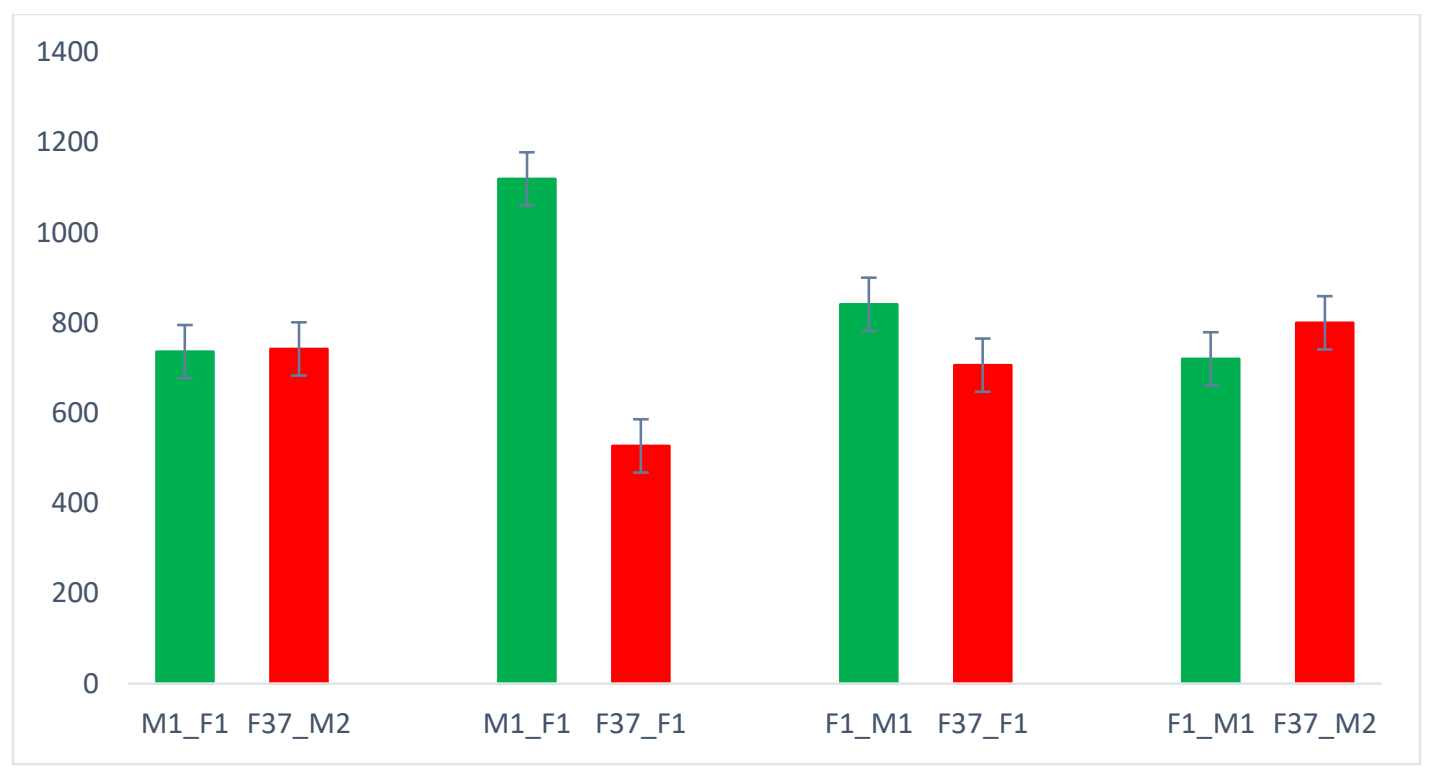

Supplementary Fig 2: A) Paternity/fitness test using 10 virgin wildtype females crossed with 5 each of the 2 different transgenic males (B) After 4 days the males were removed and the females transferred on new flour and the females were left for 21 day and then removed and the offsprings sired were counted and recorded. 
Supplementary information 3a: piggyBac insertion in T. casteneum line F1_M1 piggyBac insertion in the first intron of Tc007038 gene. The bold TTAA sequence represents the site of piggyBac integration.

F1_M1 line = pBac $\left\{3 \times 3\right.$ 3_EGFP_SV40-attP_B2t $\left.(1 \mathrm{~Kb}) \_D s R e d \_S V 40 a\right\}$

TGCAAAAAAATACATTGGCTAACAAAAGCAAACGATTTTACTATAATTTTTACTC TTTGTTTCACATAAAATATAAATTCTCTCTAACTTTTACTATAAAAGCATTTACTT AACTTTATTCATACGGCTCATTATTTCTATGAACAGTTAATTCCTGCTTTGAAATG CTATGATTATATCCGGTAGGCATTTTAATATTACGTCACCTTAATCACTTTAATGA TTTATTTGTCTTATTTTTTGTGTTAACAATAATTAAATAAATGCATTTTGTTTTTGT TATAATTAGGATTAGAACAATTATTATTTTATTTTAAACAATTTAAACAAGT TTAA-5'PiggyBac3' -

TTAATTAATATTAAAAAAATGTTGTTTTTTCCAGCGATTCAATTAAGGGATTAATT ATAAAAACAGTATGCTATGTACTCGTATTTAGGAGATAAACTCTGGATCCGGTGC TCGTAATGATAACAAAAACGGATATTAAATTTCTGATAAGCAGCAATAAAACCT AAT (5' Junction)

Note: integrated @ TC007038 first intron.

Supplementary information 3b: piggyBac insertion in T. casteneum line M1_F1 piggyBac insertion in the first intron of Tc012012 gene. The bold TTAA sequence represents the site of piggyBac integration.

M1_F1 line $=$ pBac $\{3 \times 3$ 3_EGFP_SV40-attP_B2t (1Kb)_DsRed_SV40 $\}$

TGGTAAATAAGTCCAACATTTTTGAAATAATTTTTATTAGAAAAAAAAGCACAAA AAGTTAATCTTTGCACATCAATCACGATCCTTAATTCTTGTAATAAATTGTTTCAC CAAAGAAAATAAAGTTGTTCATCCGCAAGCATGATACCACAAAAACAAGACCTC ACTTTCCCAGAATTTACAAAATATCCGCGGCATGTCATCCACAATCGCGGCAATT TAATTTCCATTTTTATCTTTTCACCGAAAGTAAATCCACTTCCTTGCACGCAAGAA AAATTGTCATTGTGACAATTTATCTCACTATCATCTCTGGTATTGTTTAATTAAAA ATTTCTCGTCGTTTTTTCACTTTCAACGACGATGATGAGTTCATTATGGAGGCCTT GGCACGTGTTTTCAAATTCGTACGCTTGGCTAG TTAA-5'PiggyBac3' $-\boldsymbol{T T A A}$ TTAAGCTAAATCAGGACCAAAACATGGCAAATATAGTCAATGTAAACACGGGCT GCTGTCCTGTCCATAATCAATATGCGTAAGATGGGTGTTCCGGTTTGGACACCTC CTTTCCGGTTTTTAATTCCCATTGCGGCAACGACCTTAGCGCGCCCAGTTTCCGGA CACAATAACGTTGGCAGACCTGCCACCATTTTACCAACTTTCT (5' Junction)

Note: integrated @ TC012012 first intron.

Supplementary information 3c: piggyBac insertion in T. casteneum line F37_F1 line = pBac \{3xp3_DsRed_SV40-attP_B2t (1Kb)_EGFP_SV40\}

piggyBac insertion in the pangolin CDS gene Tc032496. The bold TTAA sequence represents the site of piggyBac integration.

TGAATTCTTCAATAAATAAATCGTTTAATTGGCCTATCTTGATTAACTGTCATCTT GAAGTTTCAGCTACATACTTTGATTACTAGTTTGAATTAA-5'Piggy Bac3'-TTAA 


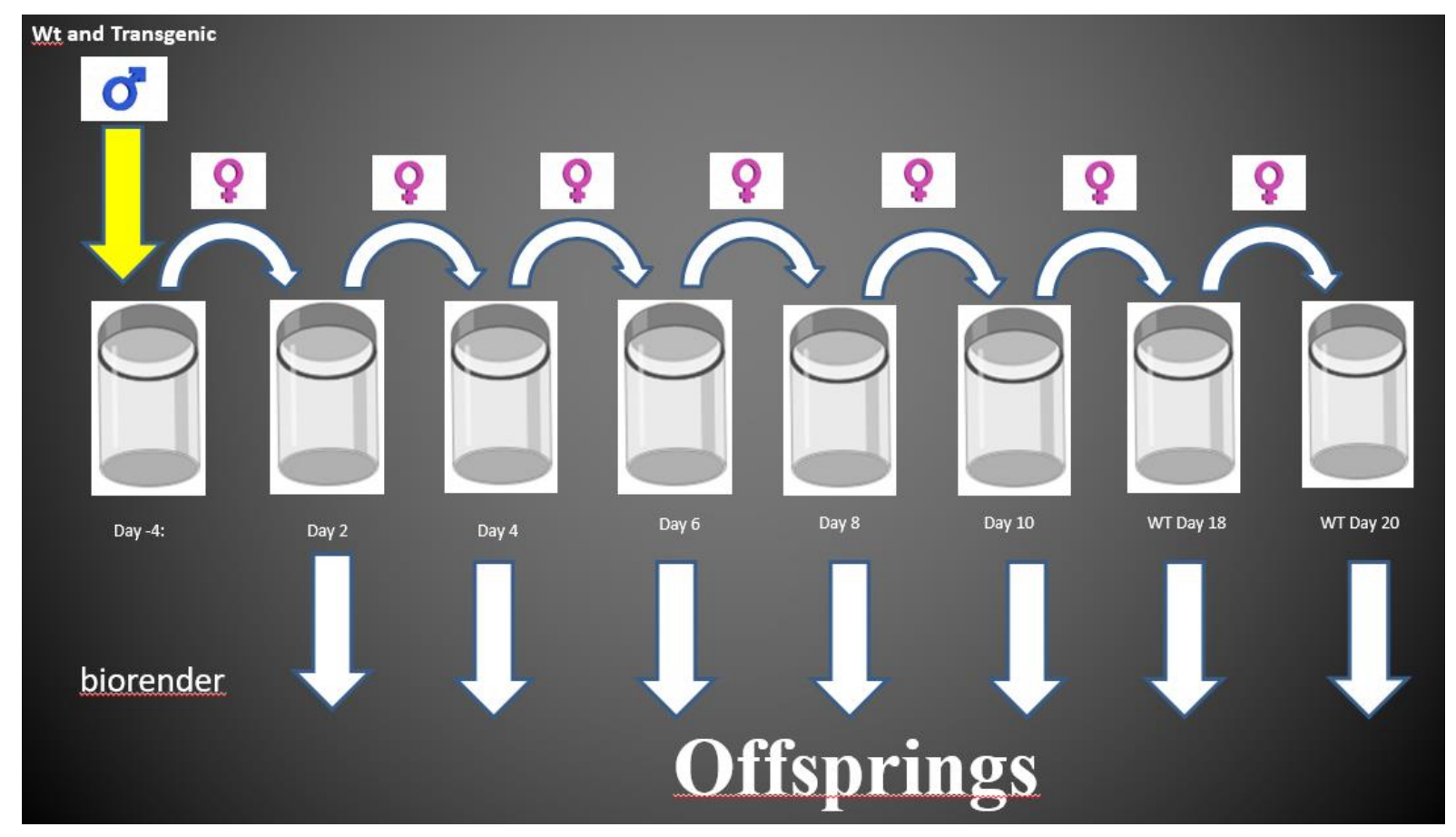

Supplementary 4: Mating/Fitness test assay experimental protocol 


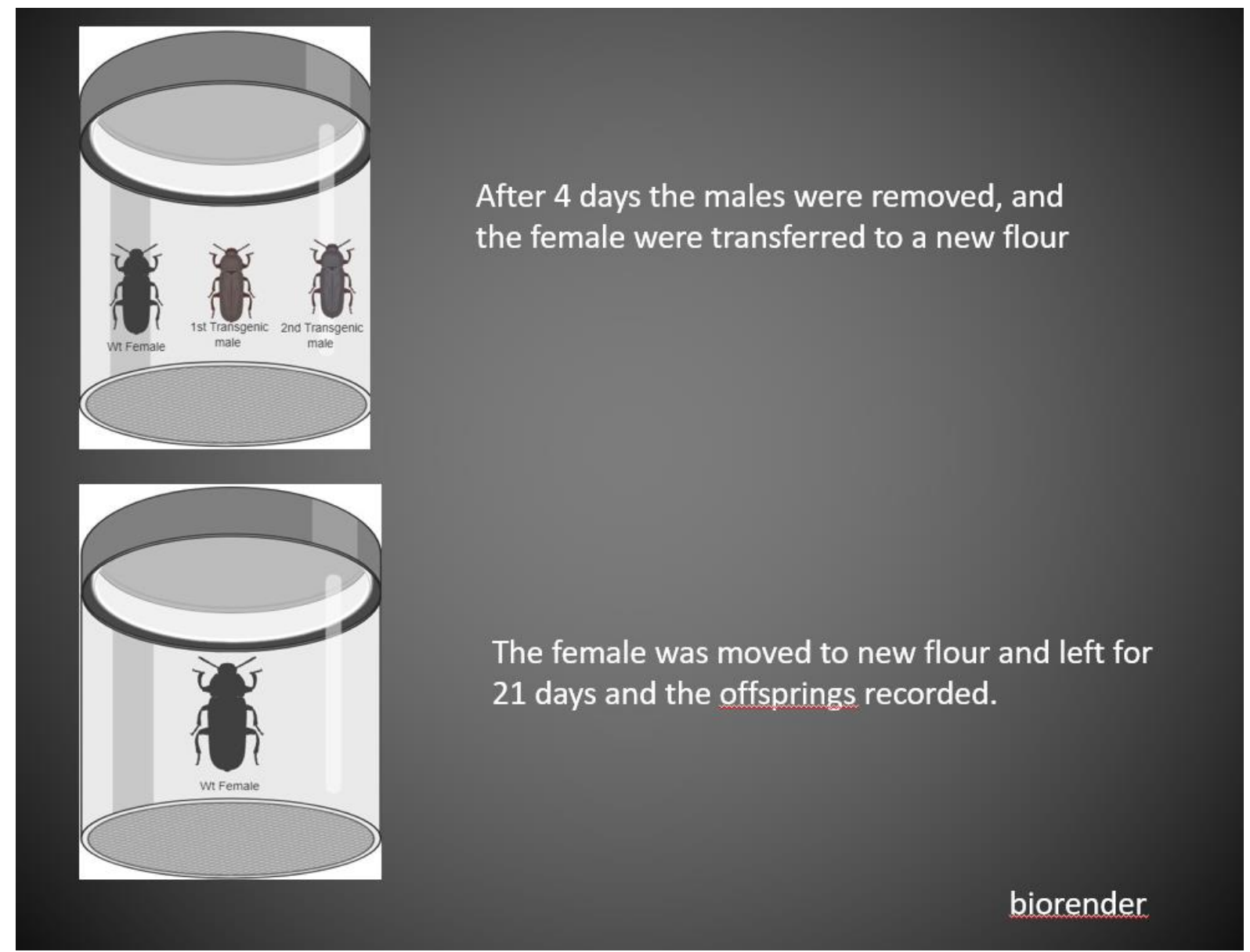

Supplementary 5:10 Virgin wildtype females crossed with 5 each of 2 different transgenic males in a paternity assay 


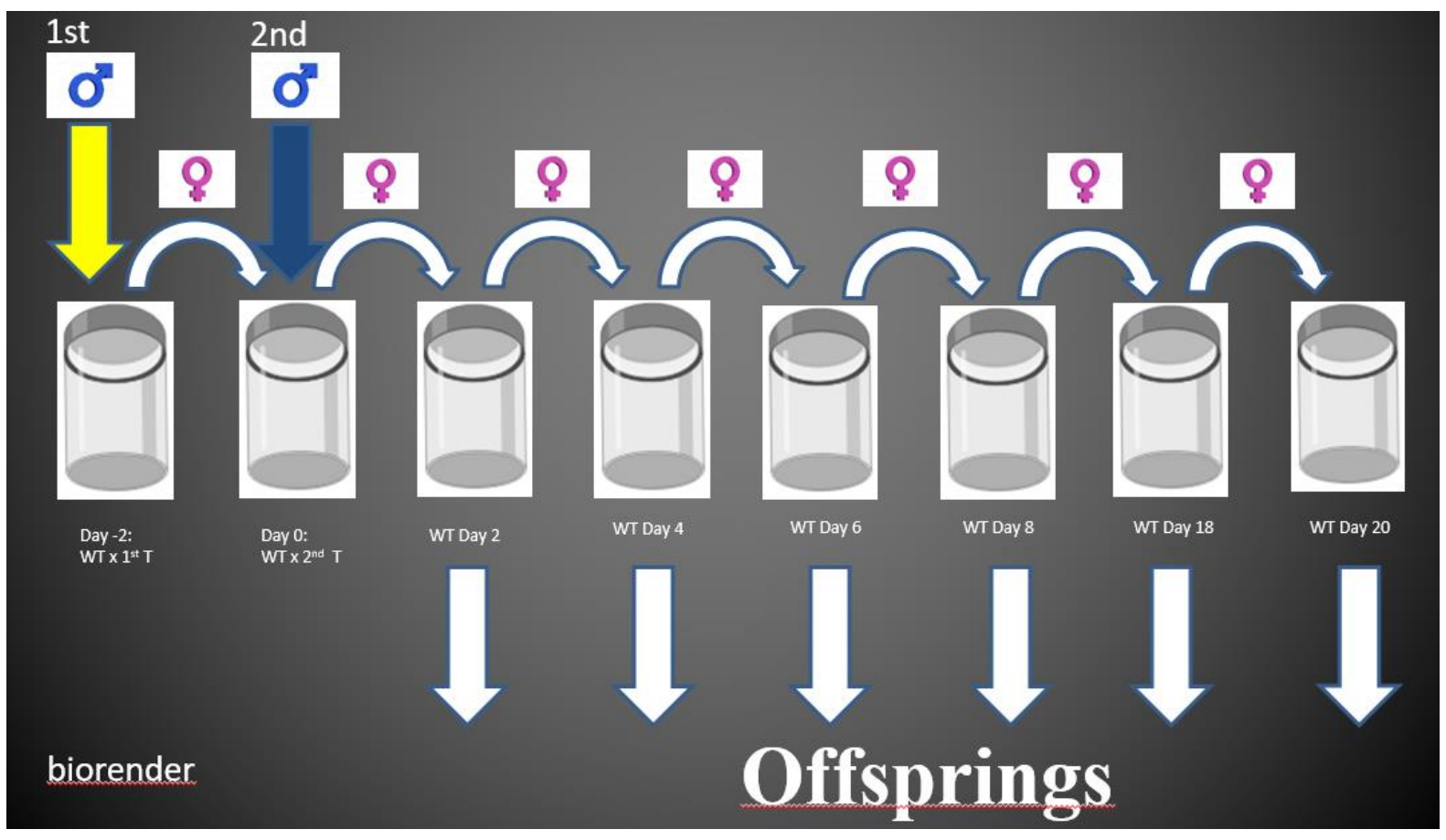

Supplementary 6:Sperm competition assay experimental protocol

Additional File 1: Supplementary Table 1: List of primers used in this experiment

\begin{tabular}{|c|c|c|}
\hline ID & NAME & Sequence $5^{\prime}-3^{\prime}$ \\
\hline MID\#4 & Tc-b2t-NcoI 1KB-F & ATGGCCATGGAGGTATCTCACAACCACCGCTTCC \\
\hline MID\#7 & Tc- b2t-ClaI-R & GAGGATCGATTTTACTTTAACGTTAAACAAATTTATTAAAGAAATACCG \\
\hline MID\#103 & ClaI-EGFP-SV40_F2 & GAGGATCGATATGGTGAGCAAGGGCGAG \\
\hline MID\#104 & HindIII-EGFP-SV40_R & TTTAAGCTTGATGAGTTTGGACAAACCACAAC \\
\hline No.5 & iPCR5'F1 & GACGCATGATTATCTTTTACGTGAC 1st round PCR \\
\hline No.6 & iPCR5'R1 & TGACACTTACCGCATTGACA \\
\hline No:7 & iPCR5'F2 & GCGATGACGAGCTTGTTGGTG 2nd round PCR \\
\hline No.8 & iPCR5'R2 & TCCAAGCGGCGACTGAGATG \\
\hline No.9 & iPCR5'Seq & CGCGCTATTTAGAAAGAGAGAG \\
\hline MFS227 & iPCR3'F1 & AGTCAGTCAGAAACAACTTTGGCACATATC \\
\hline \multirow[t]{4}{*}{ MFS228 } & iPCR3'R1 & CCTCGATATACAGACCGATAAAACACATGC \\
\hline & 3' Junction CH_PRSeq & TACGCATGATTATCTTTAACG \\
\hline & Pjet 1.2 Forward & CGACTCACTATAGGGAGAGCGGC \\
\hline & Pjet 1.2 Reverse & AAGAACATCGATTTTCCATGGCAG \\
\hline
\end{tabular}


3.2 Identification and Characterization of Potential Cellularization Gene(s) for the Development of Transgenic Embryonic lethality system in Tribolium castaneum

Female Embryonic Lethality System (FELS) is an essential and an integral part of any SIT program because this is where female embryos are terminated at very early life stage before hatching. The transgenic approach can be used to mimic and replace the use of the classical radiation SIT technique. Thus, in this chapter we outline the process we undertook to develop the system for a coleopteran insect pest; firstly we search the Tribolium genome for $D$. melanogaster early embryonic cellularization genes orthologs with an intention of transferring the system already established in some dipterans insect pests to Tribolium, but unfortunately we did not find any conserved orthologs from the Tribolium genome database. We then search for candidate genes in stage specific transcriptome data, and the iBeetle unbiased phenotypic screen approaches where we identified only Tc007675 gene. The new Tc007675 early cellularization gene we discovered was verified via Whole Mount in Situ Hybridization (WMISH). After which, we amplified the upstream region of the gene, and used it to generate driver line which is used to mediate the expression of tTA in embryo at cellularization stage.

ISAH, Musa Dan'azumi, Bibi Atika \& Ernst A. Wimmer

\section{Authors contribution to the practical work}

ISAH, Musa Dan'azumi: Conceived and design the experiment, conducted all the other experiment and analysis. Wrote the manuscript.

Bibi Atika: Conducted the parental RNAi microinjection, cuticle prep analysis and documentation.

\section{Status: Work in progress}


Identification and Characterization of Potential Cellularisation Gene(s) for the Development of Transgenic Embryonic lethality system in Tribolium castaneum

ISAH, Musa Dan'azumi, Bibi Atika \& Ernst A. Wimmer

1) Department of Developmental Biology, Johann-Friedrich-Blumenbach-Institute of Zoology and Anthropology, Göttingen Center for Molecular Biosciences, Georg-August-University Göttingen, 37077 Göttingen, Germany.

2) Department of Crop Science, Faculty of Agriculture, Wildlife and Forestry Resources Management, University of Calabar, P.M. B. 1152, Calabar, Cross River State, Nigeria.

* Corresponding Author: E. A. Wimmer. Tel. +49 5513928666

E-Mail address: ewimmer@gwdg.de.

\section{Abstract:}

The Sterile Insect Technique (SIT), is an environmentally friendly and acceptable pest management strategy which has been applied effectively in the management of several dipteran insect pests in the wider context of Integrated Pest Management approach. However, none of such transgenic approach has so far been reported for the management of coleopteran insect pests, even though they constitute about $40 \%$ of all described insect species, with most of them reported as important key pest of agricultural importance. SIT approach comprises different components such as sperm marking for monitoring, embryonic lethality and sexing for male only released. However, for some of these systems to be effective there is a need to determine an early embryonic gene (s) that can cause embryonic lethality at stage specific period, and which can also be employed for the generation of lethality in a sex specific manner for male only releases. Thus, to develop the system in Tribolium being the model for coleopteran insect pests, we search for the orthologs of early cellularization gene of dipterans such as slam, serendipity $\alpha$, nullo, bottleneck in the Tribolium castaneum genome database, but we did not find any conserved orthologs in T. castaneum.

Therefore, we searched for early embryonic genes of $T$. castaneum from generated embryo transcriptomic data, and the iBeetle unbiased phenotypic screen to determine possible cellularization genes, where 10 and 5 possible gene candidates were identified respectively. Using Whole Mount In-Situ Hybridization (WMISH) procedure, we identified Tc007675 gene that shown expression in a cellularization specific pattern from the 15 potential candidates. 
After the identification, $1.4 \mathrm{~Kb}$ and $2.1 \mathrm{~Kb}$ Promoters/Enhancers (P/E) of Tc007675 gene from Tribolium DNA were isolated and subsequently cloned to mediate the expression tTA in the cellularization specific stage. The constructs generated were injected via germline transformation, and several transgenic individuals were generated. Using WMISH, we observed M63_F1 (1.4Kb) and F37_F1 $(2.1 \mathrm{~Kb})$ generated driver strains showed the transient expression of tTA, which is consistent with the endogenous gene expression of Tc007675. The driver strains hence provided us with a possible candidate to use for both embryonic lethality and sexing systems for an SIT approach in Tribolium casteneum

Keywords: Tribolium castaneum, cellularization, embryonic lethality, sexing, TC007675 


\section{INTRODUCTION:}

The Sterile Insect Technique (SIT), is a specie specific and environmentally acceptable pest management approach ${ }^{1}$, that is being applied as an alternative to the current control measures used against dipterans, lepidopterans and other group of coleopteran insect pests. Currently control measures rely predominantly on the application of insecticides ${ }^{2}$ aside other control measures, which present some problems such as facilitating rapid emergence of insecticide resistance ${ }^{3}$ by many organisms. Also, chemical application of insecticides is not always compatible with other environmentally efficient management practices, and also have adverse effect on environment and human health ${ }^{4}$. SIT on the other hand is a species-specific ${ }^{5}$ and safe for the environment and human health ${ }^{6}$ approach. It is also safe when in combination with organic farming ${ }^{78}$ in Integrated Pest Management (IPM) programs. More so, the released insects being mobile can actively seek conspecific individuals for mating.

Classical SIT was used successfully in controlling tsetse-fly in Zanzibar ${ }^{3}{ }^{9}$ and new screw worm ${ }^{10}$ in the USA and Libya ${ }^{11}{ }^{12}$. It involved the large production of reproductively radiated sterile males ${ }^{5}$, with the females removed, prior to release. However, one of the major drawback of sterilization process by ionizing radiation is that it reduces the competitiveness ability of the sterilized males ${ }^{13}$ due to its effects on somatic cells, and thus required repeated large releases of males in large numbers to inundate the pest population for effective result to be achieved.

Moreover, sterile male-only releases is effective ${ }^{14} 15$, and with removing useless but potentially harmful females from the last mass-rearing step at early embryonic stages also saves costs, thus highlighting the necessity for an efficient sexing mechanism.

Transgenic approaches of introducing three important traits have been employed in several insect strains to enhance their use in SIT ${ }^{13}$ and replace the classical approach: the strategies employed are firstly, a female specific lethality system ${ }^{15}{ }^{16}$, secondly an embryonic lethality system ${ }^{17}$ to substitute classical SIT irradiation procedure; and thirdly, transgenic marker ${ }^{18}$ systems, and this has substantially resolved the drawbacks of classical genetic SIT approach.

Transposon-based germline transformation have been used for the development of many transgenic organisms. To provide a transgene-based embryonic lethality ${ }^{19}$ and sexing ${ }^{20}$ systems in the model fruit fly D. melanogaster ${ }^{21}$, Mosquitoes ${ }^{22}$ and Mediterranean fruit pest Ceratitis capitata ${ }^{20}$, early cellularization gene such as slam, serendipity $\alpha^{23}$, nullo, ${ }^{124}$ bottleneck ${ }^{1}$ are needed and have being used to achieved such goals, with however paucity of data on the use of the strategy for coleopteran insect pests despite them constituting about $40 \%$ 
of the class Insecta. The reason probably is that there is little or no concerted effort to determine early cellularization genes in coleopteran insect pest for functional studies that will enable the adoption/development of the SIT technology for this order of insects.

iBeetle is an unbiased candidate screening procedure in a non-dipteran insect species ${ }^{31}$, which involves the Knocking down of any phenotypes by dsRNA injection into larvae or pupa ${ }^{31}$. The parental RNAi effect is systemic ${ }^{34} 35$ and spreads in all cell of the injected animal, which is then transferred to the offspring ${ }^{31} 34$. RNAi have been used extensively in gene modification for various functional studies ${ }^{40}$.

Therefore, there is the need to adopt the novel and improved biological strategies to other insect pest such as the Red Flour Beetle (RFB) i.e. T castaneum apart from the dipterans. In this respect, we present the first report of an early embryonic cellularization ${ }^{25}$ gene that is used for functional studies in the expression of tTA for the development of driver lines, and its subsequent applicability for the development of female specific/embryonic lethality and transgenic sexing systems in Tribolium casteneum.

\section{RESULTS:}

\section{Analysis of embryonic transcriptomic data}

To develop a transgenic embryonic lethality and sexing systems for coleopteran. We analyzed transcriptomic data that have been used for a better genome annotation ${ }^{26}$, and using defined $\log _{2}$ RPKM maternal, $\log _{2}$ RPKM tcas1 (3-4 hours), $\log _{2}$ RPKM tcas2 (9-10 hours) and $\log _{2}$ RPKM tcas3 (10-11 hours) from the transcriptomic data. We identified 10 genes from the transcriptomic data that are higher expressed at 9-10 hours based on high read counts (Reads Per Kilobase of transcript Per Million (RPKM) mapped reads) compared with other stages. 1011 hours stage have reduce read count and out of the cellularization window period, hence not suitable for selection, also the reads recorded in tcas 1 (3-4 hours) window periods are possible maternal deposited transcripts thus made them unsuitable for selection. We also selected 5 genes from the unbiased systematic reverse genetics with uniform and/or differentiated blastoderm ${ }^{27}$ (Fig: 1) phenotype and with $\geq 80 \%$ penetrance from the iBeetle unbiased candidate screening database.

Thus, a total of 15 genes were selected following the stated criteria above, and thus set for the further analysis (Fig:2). We further determine the availability of the gene (s) transcripts, conserved domain, orthologs, homolog, gene ontology, molecular and biological functions of the selected gene(s) as shown in Table 1. 


\section{Expression patterns of 15 selected potential cellularization specific genes}

To investigate the expression pattern of 15 selected gene, WMISH was conducted using DIGRNA labelled probe generated for each gene on 0-24 hours old SB embryos as previously described ${ }^{28-30}$. The result showed varied types of expression patterns in most of the genes observed. Hence, no other gene showed a potential cellularization specific expression pattern apart from Tc007675, where expression pattern is ubiquitous in the cytoplasm at the time of cell cellularization (Fig: 3a and 3b) as observed in Drosophila melanogaster ${ }^{21}$, Ceratitis capitata $^{17}$ and $D$. suzukii ${ }^{30}$. Thus, based on the various genes expression patterns observed from the WMISH and potential based on the transcript noticed in the data during screening, 7 (Tc033639, Tc016359, Tc013978, Tc007675, Tc009479, Tc013428 and Tc008888) genes were selected to further verify the expressions after the recovery of 5' and 3'UTR of the individual genes.

\section{Rapid Amplification of cDNA ends (RACE) 5' and 3' In-Situ Hybridization of the selected genes for a cellularization specific expression after RACE PCR}

Since i was dealing with a new set of genes, with most of them having no identified molecular or biological functions, and to validate the endogenous gene expressions observed in the WMISH done in the earlier experiment. I performed a RACE PCR to amplify the 5' and 3' UTR's of the identified genes which might encode conserved domains of the 7-potential identified cellularization genes (Tc033639, Tc016359, Tc013978, Tc007675, Tc009479, $T c 013428$ and $T c 008888$ ). Moreover, the expectation is that with the additional information on the mRNA, with potential conserved domain and necessary regulatory elements recovered from 5' and 3' UTR's, the gene expression patterns could be enhanced with/or from the previously observed patterns.

We successfully recovered and amplified the 5' and 3' junction of the genes which we cloned in PCRII, and the sequences verified by nucleotides blast search on the Tribolium Genome Browser (5.2) to obtain the regions. These sequences were aligned and the identified 5' and 3' UTR's of the potential genes genomic were retrieved. The expression patterns of the genes (Fig: 4) observed were consistent to some of those observed in the WMISH earlier conducted without the 5' and 3' recovered junctions (Fig: $3 \mathrm{a}$ and $3 \mathrm{~b}$ ). We therefore report that the TC007675 gene gives a cellularization specific expression (Fig: 3a and Fig: 4) pattern as observed in D. melanogaster ${ }^{21}$, Ceratitis capitata ${ }^{17}$ and D. suzukii ${ }^{31}$ embryos. 


\section{Stage specific expression pattern of $T c 007675$ :}

To verify the developmental period at which the expression of the gene is observed, a stage specific WMISH was conducted on 0-12, 12-24- and 24-48-hours old embryos (Fig: 5). The expression pattern was detected at the cellularization stage. No expression was observed in earlier embryos stages, and in embryos ranging from 12-24hours, 24-48hours and 48-72hours old (Fig: 5).

\section{Expression pattern of Tc007675 in RNAi and Wildtype embryos using Tc007675 antisense probe}

To test specificity of probe for detection of Tc007675 and the efficiency of the knockdown and gene expression on RNAi embryos. We performed WMISH using DIG-labelled RNA Tc007675 antisense probe against 0-24 hours old RNAi embryos injected with Nonoverlapping, original IB fragments (Fig 6), DsRed injected and non-injected wildtype embryos serve as control. No expression patterns were detected in the IB and Non-overlapping injected RNAi embryos, while in the DsRed injected and non-injected wildtype embryos specific cellularization expression pattern was detected in the cytoplasm (Fig: 6). Thus, the result also show that the expression pattern is specific as no expression was noticed on the knockdown RNA interference embryos.

\section{Adult survival rate and cuticle formation in an RNAi experiment:}

To determine the function of Tc007675, we knocked down the gene by RNA interference using the iBeetle fragment and NOF, where we recorded the $v^{w}$ survival percentage in the injected female pupae. We found out that about $95.24 \%$ and $91.67 \%$ of female pupa injected with IB and NOF fragment respectively survived to adult stage (Table 2), also in the control injection with DsRed dsRNA 92\% survival rate was recorded. Thus, it signifies that the silencing of the gene expression by RNAi did not result in lethality.

To determine the RNAi effect on the development of the embryo, we performed a cuticle prep analysis of different strains i.e. San Bernadino, vermillion ${ }^{\text {white }}$ and Pig19. The result showed that apart from vermillion ${ }^{\text {white }}$, which showed no obvious deformation in relation to the DsRed injected organisms, the San Barnadino and PBA19 strains showed reduced and malformed cuticles which could be attributed to difference in their genetic background. 


\section{Generation of blastoderm specific driver strains:}

To generate a driver strain that will lead to expression of a heterologous transcription factor in a tet-off binary system. Using the tetracycline-controlled transactivator (tTA) acting as part of the driver strain, where an early embryonic gene Promoter/Enhancer region is to be used for the driver construct. Thus, we successfully isolate $1.4 \mathrm{~Kb}$ and $2.1 \mathrm{~Kb}$ Promoter/Enhancer $(\mathrm{P} / \mathrm{E})$ regions of the early embryonic gene Tc007675 (Fig: 7).

Using the amplified early embryonic gene Tc007675 P/E regions. I successfully cloned it upstream of tTA, and with that $\mathrm{i}$ generated 2 constructs i.e. pBac \{3xP3_EGFP_SV40 a_attP_TC007675 (1.4Kb)_tTA_SV40\} (Fig: 7b) with the $1.4 \mathrm{~kb}$ P/E region, and pBac \{3xP3_EGFP_SV40 -a_attP_TC007675 (2.1Kb)_tTA_SV40\} (Fig: 7c) with the $2.1 \mathrm{~kb}$ P/E region. With these constructs, i generated driver strains by piggyBac germline mediated transformation that efficiently and transiently drive tTA at an early blastoderm stage of development.

\section{Detection of blastoderm specific expression mediated by driver lines.}

0-24 hours old embryos were collected from three 1.4Kb P/E (M63_F1, M11_M2 and M67_M1) and three 2.1Kb P/E (M6_M1, M23_F1 and F37_M1) different transgenic driver lines individually and they were tested for tTA expression by WMISH with a anti sense RNA probe against tTA. The result of the in-situ showed that only M63_F1 (1.4Kb) and F37_F1 $(2.1 \mathrm{~Kb})$ (Fig: 7) lines showed an expression consistent with the endogenous gene expression as shown by Tc007675 probe (Fig 3a and 4). Other lines, however, did not show this expression.

\section{Examination of $T c$-transformer intron to cause female specific protein biosynthesis:}

To assess the effector and sexing lines (Fig: 8a/8b) for mediation of female-specific lethality and embryonic lethality systems. The candidate pBac 3xP3_EGFP_SV40 -a $\{$ attP_TC007675_1.4kb_tTA_SV40\} M63_F1 driver transgenic strain was crossed with pBac \{3xP3_DsRed_SV40_TRE_hsp68 _tGFP_SV40\} (Fig: 8a) and/or pBac \{3xP3_DsRed_SV40_TRE_hsp68_Tc-intron_tGFP_SV40\} (Fig: 8b) effector and sexing strains respectively. The embryos obtained were analyzed under fluorescent microscope for the determination of green fluorescence protein (Fig: 9a and 9b).

The results show that some effector strains F1_M4, F5_F4, F11_M1, F16_M1, M10_M3 and M7_F2 showed no green fluorescence protein in the embryos, while, F20_M1showed weak 
green fluorescence. In contrast, the wildtype embryos show no green fluorescence protein expression (Fig: 9a). In a like manner, the result of the sexing crosses showed strong green fluorescence protein expression in the transgenic F35_F1 and M11_M3 embryos which was not observed in the wildtype embryos (Fig:9b) embryos.

Thus, the preliminary conclusion that can be deduces from the experiment is that the driver line is effective, and it mediates the expression of the effector responder lines. Which can be used for the development of embryonic lethality system. Also, we attempted to examine if expression can be effected in a female specific manner in order to generate potential sexing lines (Fig:9b). However, the result of the experiment is so far inconclusive and more in-depth experiments are needed.

\section{MATERIALS AND METHOD:}

\section{Beetle stock and rearing:}

Red Flour Beetle (RFB), Tribolium castaneum vermillion ${ }^{\text {white }}\left(v^{w}\right)$ strain was used in all experiments except otherwise stated. Beetle stocks were fed on full grain flour supplemented with $5 \%$ yeast powder in 4.5 liter square plastic boxes $(15 \mathrm{~cm} \times 15 \mathrm{~cm} \times 20 \mathrm{~cm})$, made with ventilation orifice on the lid and were kept at $32{ }^{\circ} \mathrm{C}$ with constant light ${ }^{32}$.

\section{Germline transformation strains:}

All germline transformation for the generation of driver line, sexing effector and embryonic lethality reporter strains constructs were injected into 1000 (0-2hrs old) embryos each using the following concentrations: piggyBac constructs $500 \mathrm{ng} / \mu \mathrm{l}$ in injection buffer mixed with 300 $\mathrm{ng} / \mu \mathrm{l}$ helper plasmid mhypBase ${ }^{33}$. Borosilicate capillaries (Sutter Instrument, USA) used for the embryonic injection were pulled with a P-2000 micropipette puller (Sutter Instrument, Novato USA), and the generated needles were open using bevellor (Bachhofer Laboratoriumsgeräte; Reutlingen, Germany).

After injection, the embryos were transferred onto an apple agar plates and sealed in a plastic box which was kept in an incubator set at $32^{\circ} \mathrm{C}$ for 2 days. After 2 days, the lid of the box containing the injected embryos were opened and kept until hatching. Hatched larvae were picked with a hair thin brush individually and placed on full wheat flour until pupation. After pupation, the pupae $\left(\mathrm{G}_{0}\right)$ were sexed and individually crossed to 3 non-injected individuals of 
the opposite sex. Identified $F_{1}$ transgenic offspring obtained were further individually outcrossed with three $v^{w}$ wildtype individuals of the corresponding sexes.

Identification of potential early embryonic gene(s) from tissue/stage specifically embryonic transcriptomic data:

Over 9800 tissue/stage specific embryonic transcriptomic data 26 (https://www.ncbi.nlm.nih.gov/bioproject/PRJNA275195) of potential early embryonic specific genes comprising of maternal transcripts (SRX1396874: mRNA extraction (unfertilized eggs)), transcript expressed within 3-4 hours old embryos (tcas1: SRX1396595: mRNA extraction (pre-blastoderm)), 9-10 hours old embryos (tcas2: SRX1396600: mRNA extraction (blastoderm)) and 10-11 hours old embryos (tcas3: SRX1396873: mRNA extraction (differentiated blastoderm) in Read per Kilobase Per Millions (RPKM) were mined. A total of 10 genes (Table 1) screen for genes necessary for the development progression beyond the syncytial blastoderm stage were identified after this analysis (i.e. Tc007675, Tc008891, Tc008888, Tc004200, Tc016359, Tc033828, Tc034961, $T c 007021, T c 034963, T c 013428$ ) from the tissue/stage specific embryonic transcriptomic data and also, based on analysis of expression levels in RPKM. Their transcript lengths, identification numbers and fly orthologs were obtained (Fig 2).

Identification of potential early embryonic gene(s) from iBeetle systematic reverse genetics empty egg phenotypes data:

Over 1500 systematic reverse genetics empty egg phenotypes data were obtained from the genome wide unbiased screening genes in the iBeetle project (Fig 2). A total of 5 genes (Table 1) screen from systematic reverse genetics empty egg phenotypes (Tc032639, Tc032065, Tc013978, Tc009479, Tc011994) based on uniform and/ differentiated blastoderm (Fig: 1) phenotype and $80 \%$ penetrance were identified.

\section{Gene Ontology (GO) of identified potentially early embryonic stage specific genes:}

The 15 selected genes ID/iB number were blast using the iBeetle-base (http://ibeetle-base.unigoettingen.de), Genome-Browser (http://bioinf.uni-greifswald.de/blast/tribolium/blast.php), fly base (http://flybase.org) and NCBI (https://www.ncbi.nlm.nih.gov), data bases to determine the gene(s) transcripts, conserved domain, orthologs, homolog, gene ontology (molecular and biological functions) of the potentially early embryonic stage specific gene(s) (Table 1) 


\section{RNA Isolation:}

RNA was isolated from 0 - 72 hours and 0 - 24 hours old San Bernardino (SB) wildtype embryos for cDNA synthesis or RACE-PCR respectively using ZR Tissue and Insect RNA RNA mocroprep Kit (ZYMO RESEARCH Catalog No. R2030). The RNA extracted was treated with TURBO ${ }^{\mathrm{TM}}$ DNase in column digestion to remove any residual DNA contamination. The TURBO ${ }^{\mathrm{TM}}$ DNase was inactivated using the DNase inactivation reagent as described in TURBO DNA-free ${ }^{\mathrm{TM}}$ kit Ambion; AM1907' manual. The RNA concentration was subsequently measured and kept in $-80^{\circ} \mathrm{C}$ freezer until use.

\section{cDNA Synthesis:}

After RNA isolation and measurement, $1 \mu \mathrm{g}$ of the RNA from the $0-72$ hours old embryo was used for the first strand cDNA synthesis using Thermo Scientific Maxima First Strand cDNA synthesis kit for RT-qPCR. Generated cDNA was kept at $-80^{\circ} \mathrm{C}$, and used later as template for the amplification of specific and selected gene sequences by PCR.

\section{Amplification of the proposed 15 cellularization specific gene sequences:}

To isolate the different selected genes, we use the generated cDNA and their sequences were amplified using designed gene specific primers (Supplementary table: 1) of the individual genes. This is done on the basis of the transcript obtained from iBeetle web server (http://ibeetle-base.uni-goettingen.de/) and/or Tcas 5.2 Tribolium gbrowser (http://bioinf.unigreifswald.de/gb2/gbrowse/tcas5/) and using Basic Local Alignment Search Tool (BLAST) ${ }^{34}$. Primers sequences were designed and synthesized by Eurofins MWG Operon (Ebersberg, Germany).

A $50 \mu 1$ PCR mixture consisting of water $29 \mu 1$, cDNA $1 \mu 1$, 5x Phusion buffer 10 $\mu 1$, Phusion polymerase $1 \mu 1$, dNTPs $5 \mu 1$, forward primer $2.5 \mu 1$ and reverse primer $2.5 \mu 1$ in a PCR program of $2 \mathrm{~min}$ at $98^{\circ} \mathrm{C}, 30 \mathrm{sec}$ at $98^{\circ} \mathrm{C}, 30 \mathrm{sec}$ at varying temperatures, $2 \mathrm{~min}$ at $72^{\circ} \mathrm{C} 35$ cycles, 10 min at $72^{\circ} \mathrm{C}$ were conducted in a thermocycler for each gene fragment. The amplified fragments were run on $1 \%$ agarose gel and distinct single bands for each gene were excised and purified using the NucleoSpin ${ }^{\circledR}$ Gel and PCR Clean-up Kit (Macherey-Nagel GmbH $\alpha$ Co. KG, Düren, Germany).

The blunt PCR amplified fragments obtained were cloned into Pjet 1.2 vector (Thermo Fisher Scientific, 64293 Darmstadt, Germany), using the following ligation procedure; Peg $40001 \mu 1$, T4 DNA ligase (NEB) 1 $\mu 1,10 x$ T4 DNA ligase (NEB) buffer $2 \mu 1$, Pjet 1.2 vector (50ng/ $\mu \mathrm{l})$ 
$1 \mu 1,10 \mathrm{mM}$ ATP $2 \mu 1$, and varying volume of fragment based on their concentration and water to adjust to $20 \mu \mathrm{l}$. The mixture was kept in a $16^{\circ} \mathrm{C}$ water bath overnight and afterwards $5 \mu 1$ of the mix was transformed in a DH5 $\alpha$ competent cells, which was subsequently spread on predried X-gal spread agar plates.

Positive clones obtained after colony PCR using standard primers pJet1.2_fwd and pJet1.2_rev were inoculated and further purified using NucleoSpin ${ }^{\circledR}$ Plasmid Kit (Macherey-Nagel GmbH $\alpha$ Co. KG, Düren, Germany). The purified clones were sequenced using either pJet1.2_fwd or reverse pJet1.2_rev (Additional file 1) primers based on the orientation of the cloned fragment for antisense probe. Furthermore, the fragments were amplified using Gene Specific forward Primers and Pjet 1.2 forward primer with T7 overhang (Additional file 1) to generate the antisense strand. The fragments were then in vitro transcribed using T7 RNA polymerase to generate antisense probes. Whole mount in Situ Hybridization was conducted to analysis the expressions of the individual genes.

\section{Rapid Amplification of cDNA ends (RACE).}

RNA was isolated from the 0-24 hours old embryos; the RNA was used for the Rapid Amplification of cDNA Ends (RACE) to generate first strand of 5'_RACE_Ready cDNA and $3^{\prime}$ _ RACE_Ready cDNA libraries, using $1.4 \mu \mathrm{l}$ RNA, $1 \mu \mathrm{l} 5^{\prime} \mathrm{CDS}$ primer A, $1.35 \mu \mathrm{l}$ for 5'_RACE reaction, and 1.4 $\mu 1$ RNA, 1 $\mu 13^{\prime} \mathrm{CDS}$ primer A, 2.35 $\mu 1$ water for 3'_RACE reaction according to SMARTerTM RACE cDNA Amplification Kit (Takara Bio Europe SAS, 78100 Saint-Germain-en-Laye, France). After the reaction the products were diluted with $100 \mu 1$ Tricine-EDTA and kept at $-20^{\circ} \mathrm{C}$. The $3^{\prime}$ _ RACE_Ready cDNA and 5'_RACE_Ready first strand cDNA synthesized was used for the RACE PCR.

The Universal Primer Mix (UPM) provided with the kit and the corresponding Gene Specific Primers (GSP's) (Supplementary table: 1) was used for the recovery of either the 5' and 3' UTR's of the seven potential identified cellularization genes (Tc032639, Tc032065, Tc013978, Tc007675, Tc009479, Tc013428 and Tc011994) from generated RACE_cDNA libraries. PCR's were done to recover the fragments with the following concentrations and conditions: 2.5 $\mu 1$ RACE_Ready cDNA, $5 \mu 1$ UPM (10x), $1 \mu 1$ 10mM Smarter dNTP, $1 \mu 1$ 10mM GSP, $5 \mu 1$ 10x Advantage 2 PCR buffer, $1 \mu 1$ Advantage 2 polymerase and $34.5 \mu 1$ water in a total $50 \mu 1$ reaction mix for each of the $5^{\prime}$ and $3^{\prime}$ RACE_cDNA.

The mix were made to stand in a PCR, using touchdown PCR procedure of 5 cycles of $94^{\circ} \mathrm{C}$ denaturation for $30 \mathrm{sec}$, annealing/extension for $5 \mathrm{~min}$ at $72^{\circ} \mathrm{C}$ followed by 5 cycle of $94^{\circ} \mathrm{C}$ 
denaturation for $30 \mathrm{sec}$, annealing at $70^{\circ} \mathrm{C}$ for $30 \mathrm{sec}$ and extension at $72^{\circ} \mathrm{C}$ for $3 \mathrm{~min}$ and finally 25 cycles of $94^{\circ} \mathrm{C}$ for $30 \mathrm{sec}$, annealing at $68^{\circ} \mathrm{C}$ for $30 \mathrm{sec}$ and extension at $72^{\circ} \mathrm{C}$ for $3 \mathrm{~min}$. The PCR product was run on $1 \%$ agarose/EtBr gel and the distinct individual bands recovered were excised, purified using NucleoSpin Gel and PCR Clean-up Macherey-Nagel Kit (Ref 740609.250) and cloned into the pCRII (Thermo Fisher Scientific) vector and subsequently transformed into DH10 a competent cells and the potential positive clones based on the white/blue screening were picked for colony PCR.

$10 \mu 1$ colony PCRs reaction were conducted with the following concentration; Water $6.8 \mu 1$, 10x Phusion buffer $2 \mu 1$, dNTP $0.3 \mu 1$, M13 forward primer $0.3 \mu 1$, M13 reverse primer $0.3 \mu 1$, Phusion polymerase $0.3 \mu \mathrm{l}$ and in a 25 cycle of $98^{\circ} \mathrm{C}$ Initial denaturation for $2 \mathrm{~min}, 98^{\circ} \mathrm{C}$ denaturation for $30 \mathrm{sec}, 50^{\circ} \mathrm{C}$ annealing for $30 \mathrm{sec}, 72^{\circ} \mathrm{C}$ elongation for $30 \mathrm{sec}$ and $72^{\circ} \mathrm{C}$ final elongation for $5 \mathrm{~min}$ PCR reaction.

Amplified positive clones of the right sizes were subsequently inoculated in 5ml Lysogeny Broth (LB) with ampicillin antibiotic medium and kept at $37^{\circ} \mathrm{C}$ shaker set at $225 \mathrm{rpm}$ overnight. The plasmids were subsequently isolated using NucleoSpin Plasmid Macherey-Nagel (Ref 740588.250), and concentration measured using the Nanodrop and sequenced (Microsync seqlab) using a standard M13 primer.

\section{Sequence Analysis:}

The sequences obtained from the 5' and 3' UTR's of the potential cellularization genes were verified by nucleotides blast search on the (http://ibeetle-base.uni-goettingen.de/) and/or Tcas 5.2 Tribolium gbrowser (http://bioinf.uni-greifswald.de/gb2/gbrowse/tcas5/) and further analyzed by 'Geneious 9.2 version.

\section{Amplification of selected genes for a cellularization specific expression after RACE PCR with 5'UTR and/or 3'UTR}

Primers (Supplementary table: 1) were designed after the RACE PCR for the selected genes, and fragments were amplified including either/both the recovered 5' and 3' UTR's which were subsequently cloned in Pjet 1.2 Vector (Thermo Fisher Scientific, 64293 Darmstadt, Germany) blunt cloning. The fragments were amplified using Gene Specific forward Primers (Additional file 1, Supplementary table: 1) and Pjet 1.2 forward primer with T7 overhang (Additional file 1) to generate an antisense strand. The fragments were then in vitro transcribed using T7 RNA polymerase to generate DIG-labelled RNA antisense probes for WMISH as mentioned above. 
Whole Mount In Situ Hybridization (WMISH) using the DIG-labelled RNA antisense probe to 0-48 old embryos were performed described previously ${ }^{28-30}$.

\section{RNA interference using Tc007675 dsRNA (iB_07126) and Non-Overlapping Fragment (iB_07126_2)}

The RNAi experiment was conducted following an established protocol ${ }^{35} 36$. Female pupae injection was conducted using a FemtoJet ${ }^{\circledR}$ express device (Eppendorf, Hamburg, Germany) and borosilicate glass capillaries with an applied injection pressure of 400-800 hPa.

dsRNAs (iB_07126) (IB) (Table 2) and Non-Overlapping Fragment (iB_07126_2) (NOF) (Table 2) of the Tc007675 gene for the RNAi target gene experiments, were ordered from Eupheria Biotech GmbH (www.eupheria.com), and as positive control an amplified DsRed fragment was cloned in Pjet 1.2 and used as template. Pjet 1.2 forward and reverse primers with T7 overhangs were used to generate amplicons using standard PCR conditions. The DsRed single prominent band obtained was excised and clean using the appropriate PCR clean up kit. The product was used for dsRNA synthesis. After in vitro transcription, RNA was precipitated using isopropanol.

The RNA pellet was dissolved in injection buffer $(1.4 \mathrm{mM} \mathrm{NaCl}, 0.07 \mathrm{mM}$ Na2HPO4, 0.03 $\mathrm{mM} \mathrm{KH} 2 \mathrm{PO} 4,4 \mathrm{mM} \mathrm{KCl}, \mathrm{pH} 6.8$ ). After that it was continued for annealing to increase the percentage of double stranded RNA (dsRNA), and concentration was adjusted to $1 \mu \mathrm{g} / \mu \mathrm{l}$ desired for pupal injection.

About twenty female pupae of San Bernardino, vermillion ${ }^{\text {white }}\left(v^{w}\right)$ and pig19 each were injected with $1 \mu \mathrm{g} / \mu 1$ titers of dsRNAs of Tc007675 original gene fragment (iB_07126) (IB), Non-Overlapping Fragment (iB_07126_2) (NOF) and DsRed, to determine if there is any genetic background influence on the activity of the dsRNA. After the injection the pupae were kept at $32^{\circ} \mathrm{C}$ and left to develop for 4 days after which male pupae were introduced.

\section{Cuticle preparation}

Eggs were collected from 9-11day old injected females and used for cuticle preparation as described by ${ }^{31}$. The eggs were transferred from $300 \mu \mathrm{m}$ mesh and kept on an oil collection blocks for 4 day at $32^{\circ} \mathrm{C}$ with hatched larvae stored at $4^{\circ} \mathrm{C}$ for further analysis. The remaining embryos on the $300 \mu \mathrm{m}$ mesh were transferred $180 \mu \mathrm{m}$ mesh and washed for 3 minutes in $50 \%$ bleach twice to remove flour and the chorion. Cleaned eggs were embedded in a mixture of 
Hoyer's medium for cuticle preparation ${ }^{37}$. Also, the survival percentage of the injection was recorded.

\section{Amplification of 1.4kb and 2.1kb Promoter/Enhancer region of TC007675.}

All genomic DNA used for this experiment was isolated from a mixed sex of Tribolium castaneum vermilion $^{\text {white }}$ using NucleoSpin ${ }^{\circledR}$ DNA Insect (Macherey-Nagel).

To generate a driver line that can be used for early embryonic, female specific lethality and sexing transgenic lines. A $1.4 \mathrm{~Kb}$ and $2.1 \mathrm{~Kb}$ upstream (enhancers and regulatory) region of Tc007675 gene were amplified from Tribolium castaneum (San Bernadino) gDNA strains.

Polymerase Chain Reaction (PCR) was conducted using MID\#117/MID\#118 primers and MID\#123/MID\#120 for the amplification of $1.4 \mathrm{~Kb}$ and $2.1 \mathrm{~Kb} T c 007675 \mathrm{P} / \mathrm{E}$ regions respectively in a 50 $\mu 1$ PCR mixture consisting of water $28 \mu 1$, gDNA $2 \mu 1$ (50ng/ $\mu \mathrm{l}), 5 \mathrm{x}$ Phusion buffer $10 \mu 1$, Phusion polymerase $1 \mu \mathrm{l}$, dNTPs $4 \mu 1$, forward primer $2.5 \mu 1$ and reverse primer $2.5 \mu \mathrm{l}$ in a PCR program of $3 \mathrm{~min}$ at $98^{\circ} \mathrm{C}, 30 \mathrm{sec}$ at $98^{\circ} \mathrm{C}, 30 \mathrm{sec}$ at $61^{\circ} \mathrm{C}(1.4 \mathrm{~Kb})$ and $70^{\circ} \mathrm{C}$ $(2.1 \mathrm{~Kb}), 1 \mathrm{~min}$ at $72^{\circ} \mathrm{C} 35$ cycles, $10 \mathrm{~min}$ at $72^{\circ} \mathrm{C}$ were conducted in a thermocycler.

The amplified fragments were run on $1 \%$ agarose gel and distinct single bands for each gene were excised and purified using the NucleoSpin ${ }^{\circledR}$ Gel and PCR Clean-up Kit (Macherey-Nagel GmbH $\alpha$ Co. KG, Düren, Germany) and measured.

Two-step cloning procedure to generate driver lines using early embryonic $T c 007675$ gene $P / E$ region:

\section{Shuttle vector:}

Following a 2-step cloning procedure, the amplified fragments were cut with it NcoI/ClaI and

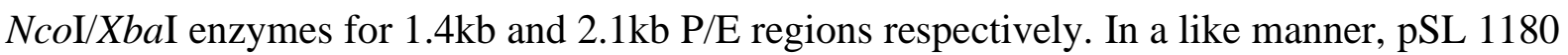
-attP-ß2t- tTA-SV40 cloning vector was cut with either NcoI/ClaI (1.4Kb P/E) or NcoI/XbaI $(2.1 \mathrm{~Kb} \mathrm{P} / \mathrm{E})$ to remove $\beta 2 \mathrm{t} \mathrm{P} / \mathrm{E}$. The reactions were kept at $37^{\circ} \mathrm{C}$ for 1 hour, after which, they were run on $1 \%$ agarose gel and distinct single bands for each fragment was excised and purified using the standard procedure.

Individual excised and purified $1.4 \mathrm{~kb}$ and $2.1 \mathrm{~kb} P / E$ regions were ligated separately to the digested pSL 1180-attP- tTA-SV40 by fusing their sticky end sites to generate pSL_af _attP_TC007675_1.4kb_tTA_SV40_af and pSL_af_attP_TC007675_2.1kb_tTA_SV40_af constructs. 


\section{Transformation vector:}

Subsequently, the cassettes af_attP_TC007675_1.4kb_tTA_SV40_af and af_attP_TC007675_2.1kb_tTA_SV40_af were excised out of the generated pSL_af_attP_TC007675_1.4kb_tTA_SV40_af and pSL_af_attP_TC007675_2.1kb_tTA_SV40_af shuttle/cloning vector constructs via AscI digestion and inserted into AscI-cut piggyBac (pBac) transposon vectors carrying 3xP3_EGFP_SV40 green fluorescent transformation eye markers to generate $p B a c$ $\{3 x P 3$ EGFP_SV40 -a_attP_TC007675_1.4kb_tTA_SV40\} (Fig; 9a) and pBac \{a_3xP3_EGFP_SV40_attP_TC007675_2.1kb_tTA_SV40\} (Fig; 9b) which were used for germline transformation.

\section{Generation of sexing effector construct by cloning Tc-tra into reporter plasmid construct:}

A 920bp fragment from the Tc-transformer (Tc-tra) containing the female sex specific spliced intron locus was amplified from SB genomic DNA by PCR using the primer 5'GTACCGGTCGCCACCATGGTAATtGGTTTTCATAGTCTTGACGAA $\quad 3$ ' (TcTcTraIn_hsp68 F) and 5'CGCTCTCGTCGCTCTCCATCTGAAATCAAGCGGTTCTCT 3' (TcTra_tGFPO R) in a $50 \mu 1$ reaction with PCR $\left(98^{\circ} \mathrm{C} 2 \mathrm{~min},\left[98^{\circ} \mathrm{C} 30 \mathrm{sec}, 66^{\circ} \mathrm{C} 30 \mathrm{sec}, 72^{\circ} \mathrm{C}\right.\right.$ $\left.1 \mathrm{~min}] 35 \mathrm{X}, 72^{\circ} \mathrm{C} 10 \mathrm{~min}\right)$. The amplified Tc-tra fragment was purified and cloned into plasmid pBac \{3xP3_DsRed_SV40_TRE_hsp68_tGFP_SV40\} which is term as the reporter construct. Cloning of the Tc-tra was obtained using megaprimer PCR cloning strategy, with 50ng/ $\mu 1$ of the vector plasmid and $150 \mathrm{ng} / \mu \mathrm{l}$ of the amplified Tc-tra as the megaprimer in a $50 \mu 1$ reaction with PCR $98^{\circ} \mathrm{C} 2 \mathrm{~min},\left[98^{\circ} \mathrm{C} 30 \mathrm{sec}, 66^{\circ} \mathrm{C} 30 \mathrm{sec}, 72^{\circ} \mathrm{C} 1 \mathrm{~min}\right] 35 \mathrm{x}, 72^{\circ} \mathrm{C} 10 \mathrm{~min}$ ) to generate pBac \{3xP3_DsRed_SV40_TRE_hsp68_Tc-intron_tGFP_SV40\} as a female sex specific reporter construct. The plasmid was sequenced (Microsync Seqlab ${ }^{38}$ ) and using Geneious' software the cloning of the Tc- transformer intron was verified.

\section{Whole Mount in situ Hybridization (WMISH):}

Whole Mount in situ Hybridization (WMISH) was done using DIG-labelled RNA antisense probe to elucidate the expression patterns of the 15 identified genes on 0-48 hours old embryo after egg lay and fixation as reported ${ }^{29}$.

Furthermore, to verify the developmental period by which the expression of the gene is determined, a stage specific WMISH using DIG-labelled probe was performed to 0-12, 12-24 and 24-48h old embryos. The Tc007675 DIG-labelled RNA antisense probe comprised the 5' 
and/or 3' UTR region(s) of the gene. 0-24 hours old embryos of wildtype, DsRed, NOF and IB fragment RNAi embryos of injected adult females were collected for WMISH using Tc007675 DIG-labelled RNA antisense probe. The expression of the gene was observed and recorded.

Embryos of $0-24$ hours were collected from $\mathrm{pBac}$ \{3xP3_Egfp_SV40_attP_Tc007675(1.4Kb)_tTA_SV40\} (M63_F1, M11_M2 and M67_M1) and pBac \{3xP3_Egfp_SV40_attP_Tc007675(2.1Kb)_tTA_SV40\} (M6_M1，M23_F1 and F37_M1) different transgenic lines, and they were tested for tTA expression by WMISH using DIG-Labelled-RNA against tTA (Ahmed et al., 2020). Staining for gene expression was perform using NBT/BCIP procedure as described ${ }^{28-30}$

\section{Test cross to evaluate driver strain and responder lines}

To validate the possibility of the generated and earlier WMISH verified M63_F1 (Fig: 9a and 10) driver strain to drive TRE embryonic and sexing responder strains. I crossed between the M63_F1 (Fig: 9a and 10) driver line to with several responders (Fig: 11a) generated lines. The crosses were made to stand for 0 - 72 hours, after which the embryos of the crosses were collected, washed for 5 minutes under a running tap water. The cleaned embryos were mounted on slide and visualized under microscopy.

\section{Microscopy:}

Image acquisition were obtained with cy3 filter, 8bit mono and black/white, and DIC filter, 24bit color and RGB settings of Zeiss Axioplan 2 microscope. Stacks were acquired and are processed with Z-projection method of ImageJ software (Version 1.47, http://rsbweb.nih.gov/ij/disclaimer.html) for some in situ and cuticle preps pictures. All figures were prepared using Adobe Photoshop (CS6).

Pictures for crosses between driver line and responder lines was documented using Zeiss Imager. Z2 equipped with 2 cameras, Axiocam 305 colour for EGFP-LP (excitation: ET480/40, emission: ET510 LP), DsRed (excitation: 533-558, emission: 570-640) 


\section{Discussion/ Conclusion:}

Sexing ${ }^{20}$ and embryonic/female specific lethality ${ }^{17}$ approaches are some of the essential components of AW-SIT program in the control of both human and agricultural insect pests. However, for the developing of sexing or embryonic/female specific lethality strains there is a need for the assemblage of the resources such as early embryonic genes ${ }^{25}$, pro apoptotic gene etc. which can be used through biotechnological engineering to mediate the various processes. Thus, with the plethora of the said resources found in dipterans and effectively established in C. capitata $^{39}$, Mosquitoes ${ }^{40}$ etc, there is however, none in coleopteran insect pest despite their huge economic impact in agricultural and human/animal health. Thus, based on this our objective is the identification and characterization of several candidate genes and their functional usage for achieving the said goal.

To engineer such strains and system in coleopteran insect pests and due to the availability of the several early embryonic cellularization development genes such as Serendipity alpha (srya) ${ }^{23}$, nullo $^{27}$, bottleneck, slam etc. in dipteran insects. I searched for the availability of the gene orthologs from the Tribolium database via protein blast search using the dipteran protein as queries. However, I could not find any conserved ortholog matching these candidate genes in Tribolium genome database.

Therefore, for us to be able to replicate the success reported in the development of biotechnological pest control strategies in other insect, we analyzed the iBeetle reverse genetics database and transcriptomic data ${ }^{26}$ to identify genes that are active during the early embryonic cellularization stage. In this process, I then identified a gene Tc007675 via WMISH and RNAi ${ }^{35}$ experiments to determine the gene expression.

However, for effective conditional suppression of pest activity by the utilization of genes in a tissue or stage specific manner, there is a need for the development of inducible binary expression system that involve driver lines that are capable of driving tTA in a tissue or stage specific manner, that will ultimately drive effector genes for a conditional drive expression strategy. I use an amplified $1.4 \mathrm{~Kb}$ or $2.1 \mathrm{~Kb}$ upstream region of the gene to mediate the expression of the heterologous transactivator (tTA) during cellularization stage which is consistent to the endogenous gene expression of the gene.

Thus, with the identification of the Tc007675 gene and also the subsequent ability of the gene to drive tTA as the endogenous expression of the gene, the driver line makes it possible to 
generate the first biotechnological approach for sexing and/or female/embryonic lethality in coleopteran insect pests as described in other dipteran insect pests. Thus, using the driver line we will be able to move towards development of biotechnological approach for the control of a coleopteran insect.

Conclusively, we can show that the Tc007675 gene can be used for driving the expression of tTA as in the endogenous cellularization expression specific pattern of the gene as shown in other dipteran species in a tissue and stage specific manner. 


\section{REFERENCES:}

1. Schetelig, M. F. \& Wimmer, E. A. Insect Transgenesis and the Sterile Insect Technique. in Insect Biotechnology (ed. Vilcinskas, A.) 169-194 (Springer Netherlands, 2011). doi:10.1007/978-90-481-9641-8_9.

2. Wojciechowska, M., Stepnowski, P. \& Gołębiowski, M. The use of insecticides to control insect pests. 13, 210-220 (2016).

3. Singh, S. \& Prakash, S. Development of resistance in Tribolium castaneum, Herbst (Coleoptera: Tenebrionidae) towards deltamethrin in laboratory. 3, 4 (2013).

4. Bernardes, M. F. F., Pazin, M. \& Dorta, L. C. P. and D. J. Impact of Pesticides on Environmental and Human Health. Toxicology Studies - Cells, Drugs and Environment (2015) doi:10.5772/59710.

5. Alphey, L. et al. Genetic technologies to enhance the Sterile Insect Technique (SIT). 8.

6. Nagel, P. \& Peveling, R. Environment and the Sterile Insect Technique. in Sterile Insect Technique: Principles and Practice in Area-Wide Integrated Pest Management 499-524 (2005). doi:10.1007/1-4020-4051-2_19.

7. Wimmer, E. A. Eco-friendly insect management. Nat Biotechnol 23, 432-433 (2005).

8. Hendrichs, M., Wornoayporn, V., Katsoyannos, B. \& Hendrichs, J. Quality Control Method to Measure Predator Evasion in Wild And Mass-Reared Mediterranean Fruit Flies (Diptera: Tephritidae). Florida Entomologist 90, 64-70 (2009).

9. Tsetse flies. https://www.iaea.org/topics/sterile-insect-technique/tsetse-flies (2017).

10. Screwworm flies. https://www.iaea.org/topics/sterile-insect-technique/screwworm-flies (2017).

11. Krafsur, E. S. (Iowa S. U. Sterile insect technique for suppressing and eradicating insect population: 55 years and counting. Journal of agricultural entomology (USA) (1998). 
12. Krafsur, E. S. \& Lindquist, D. A. Did the sterile insect technique or weather eradicate screwworms (Diptera:Calliphoridae) from Libya? J. Med. Entomol. 33, 877-887 (1996).

13. Ernawan, B., Tambunan, U. S. F., Sugoro, I. \& Sasmita, H. I. Effects of gamma irradiation dose-rate on sterile male Aedesaegypti. in 020010 (2017). doi:10.1063/1.4985401.

14. Hendrichs, J., Franz, G. \& Rendon, P. Increased effectiveness and applicability of the sterile insect technique through male-only releases for control of Mediterranean fruit flies during fruiting seasons. Journal of Applied Entomology 119, 371-377 (1995).

15. Rendón, P., McInnis, D., Lance, D. \& Stewart, J. Medfly (Diptera: Tephritidae) genetic sexing: large-scale field comparison of males-only and bisexual sterile fly releases in Guatemala. J. Econ. Entomol. 97, 1547-1553 (2004).

16. Meza, J. S. et al. Comparison of classical and transgenic genetic sexing strains of Mediterranean fruit fly (Diptera: Tephritidae) for application of the sterile insect technique. PLoS One 13, (2018).

17. Schetelig, M. F., Caceres, C., Zacharopoulou, A., Franz, G. \& Wimmer, E. A. Conditional embryonic lethality to improve the sterile insect technique in Ceratitis capitata(Diptera: Tephritidae). BMC Biol 7, 4 (2009).

18. Scolari, F. et al. Fluorescent sperm marking to improve the fight against the pest insect Ceratitis capitata (Wiedemann; Diptera: Tephritidae). New Biotechnology 25, 76-84 (2008).

19. Horn, C. \& Wimmer, E. A. A transgene-based, embryo-specific lethality system for insect pest management. Nature Biotechnology 21, 64-70 (2003).

20. Ogaugwu, C. E., Schetelig, M. F. \& Wimmer, E. A. Transgenic sexing system for Ceratitis capitata (Diptera: Tephritidae) based on female-specific embryonic lethality. Insect Biochemistry and Molecular Biology 43, 1-8 (2013). 
21. Wimmer, E. A. Applications of insect transgenesis. Nat Rev Genet 4, 225-232 (2003).

22. Catteruccia, F., Benton, J. P. \& Crisanti, A. An Anopheles transgenic sexing strain for vector control. Nature Biotechnology 23, 1414-1417 (2005).

23. Schweisguth, F., Lepesant, J. A. \& Vincent, A. The serendipity alpha gene encodes a membrane-associated protein required for the cellularization of the Drosophila embryo. Genes \& Development 4, 922-931 (1990).

24. Zhang, C. X., Lee, M. P., Chen, A. D., Brown, S. D. \& Hsieh, T. Isolation and characterization of a Drosophila gene essential for early embryonic development and formation of cortical cleavage furrows. J Cell Biol 134, 923-934 (1996).

25. Herndon, N. et al. Enhanced genome assembly and a new official gene set for Tribolium castaneum. BMC Genomics 21, 47 (2020).

26. iBeetle. http://ibeetle-base.uni-goettingen.de/.

27. Oberhofer, G., Grossmann, D., Siemanowski, J. L., Beissbarth, T. \& Bucher, G. Wnt/ $\beta$ catenin signaling integrates patterning and metabolism of the insect growth zone. Development 141, 4740-4750 (2014).

28. Schinko, J., Posnien, N., Kittelmann, S., Koniszewski, N. \& Bucher, G. Single and Double Whole-Mount In Situ Hybridization in Red Flour Beetle (Tribolium) Embryos. Cold Spring Harbor Protocols 2009, pdb.prot5258-pdb.prot5258 (2009).

29. Siemanowski, J., Richter, T., Dao, V. A. \& Bucher, G. Notch signaling induces cell proliferation in the labrum in a regulatory network different from the thoracic legs. Dev. Biol. 408, 164-177 (2015).

30. Ahmed, H. M. M., Hildebrand, L. \& Wimmer, E. A. Improvement and use of CRISPR/Cas9 to engineer a sperm-marking strain for the invasive fruit pest Drosophila suzukii. BMC Biotechnol 19, 85 (2019). 
31. Schmitt-Engel, C. et al. The iBeetle large-scale RNAi screen reveals gene functions for insect development and physiology. Nat Commun 6, 7822 (2015).

32. Handler, A. M. \& Harrell, R. A. Germline transformation of Drosophila melanogaster with the piggyBac transposon vector. Insect Mol. Biol. 8, 449-457 (1999).

33. Altschul, S. F., Gish, W., Miller, W., Myers, E. W. \& Lipman, D. J. Basic local alignment search tool. J. Mol. Biol. 215, 403-410 (1990).

34. RNAi in the Red Flour Beetle (Tribolium). http://cshprotocols.cshlp.org/content/2009/8/pdb.prot5256.short.

35. Tomoyasu, Y. \& Denell, R. E. Larval RNAi in Tribolium (Coleoptera) for analyzing adult development. Dev. Genes Evol. 214, 575-578 (2004).

36. Bucher, G. \& Klingler, M. Divergent segmentation mechanism in the short germ insect Tribolium revealed by giant expression and function. Development 131, 1729-1740 (2004).

37. Microsynth Webshop. https://srvweb.microsynth.ch/.

38. Niyazi, N. et al. Genetics and Mating Competitiveness of Ceratitis capitata (Diptera: Tephritidae) Strains Carrying the Marker Sergeant, Sr2. Ann Entomol Soc Am 98, 119$125(2005)$.

39. Alphey, L. et al. Sterile-Insect Methods for Control of Mosquito-Borne Diseases: An Analysis. Vector Borne Zoonotic Dis 10, 295-311 (2010). 


\section{FIGURES:}

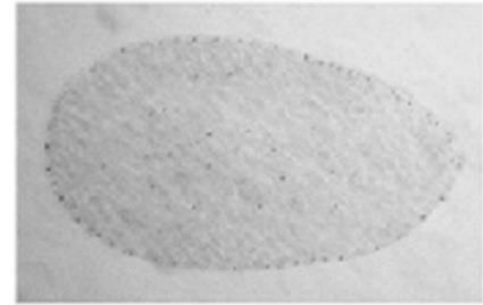

Fig 1:Uniform blastoderm ${ }^{27}$

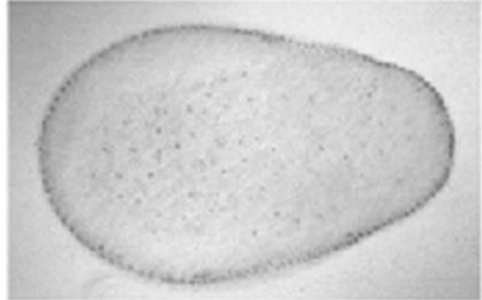

Cellularization blastoderm ${ }^{27}$ 


\section{Systematic reverse genetics}

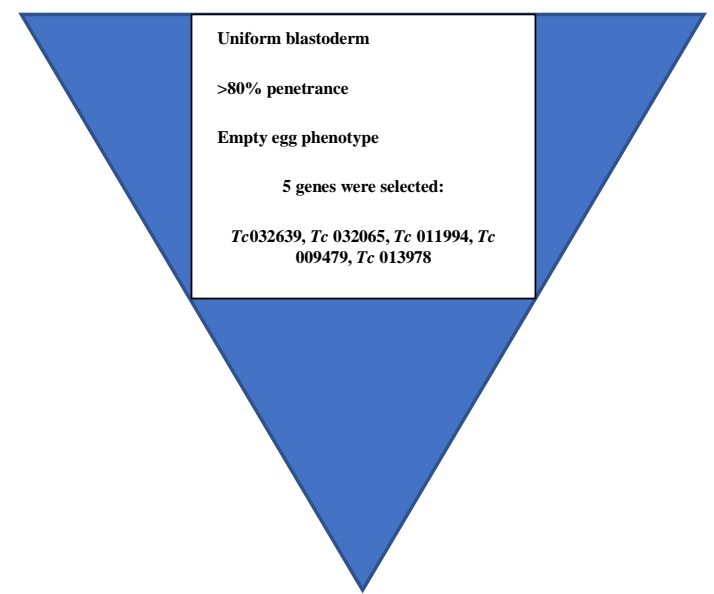

Tissue/stage specific embryonic transcriptomic data

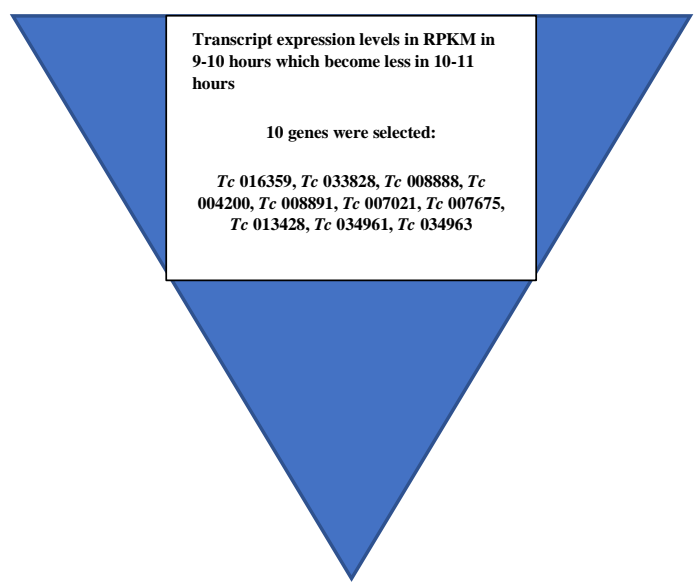

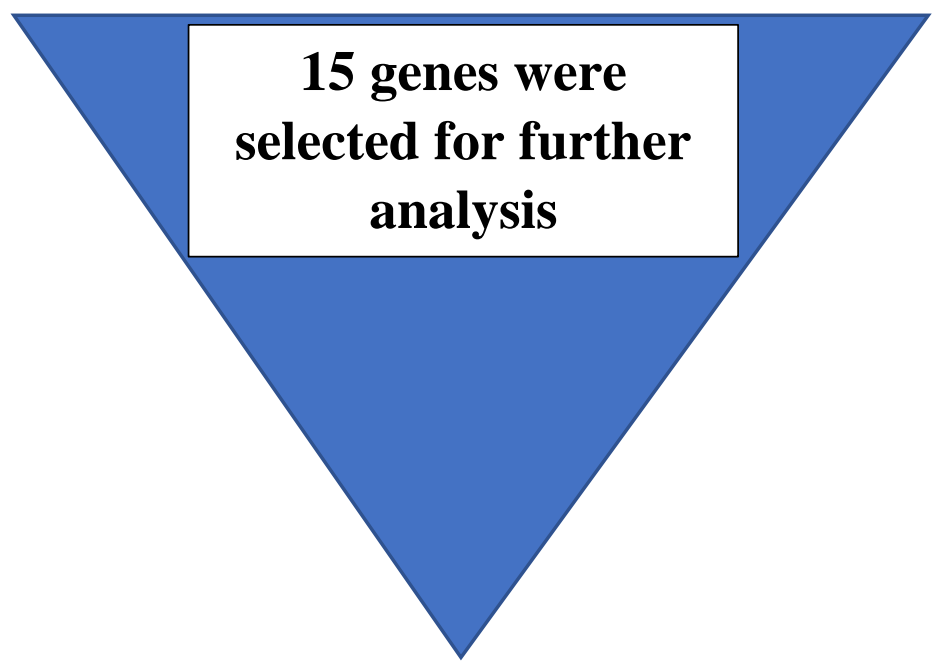

Fig 2: Diagrammatic representation of the analysis selection and list of genes for further studies 


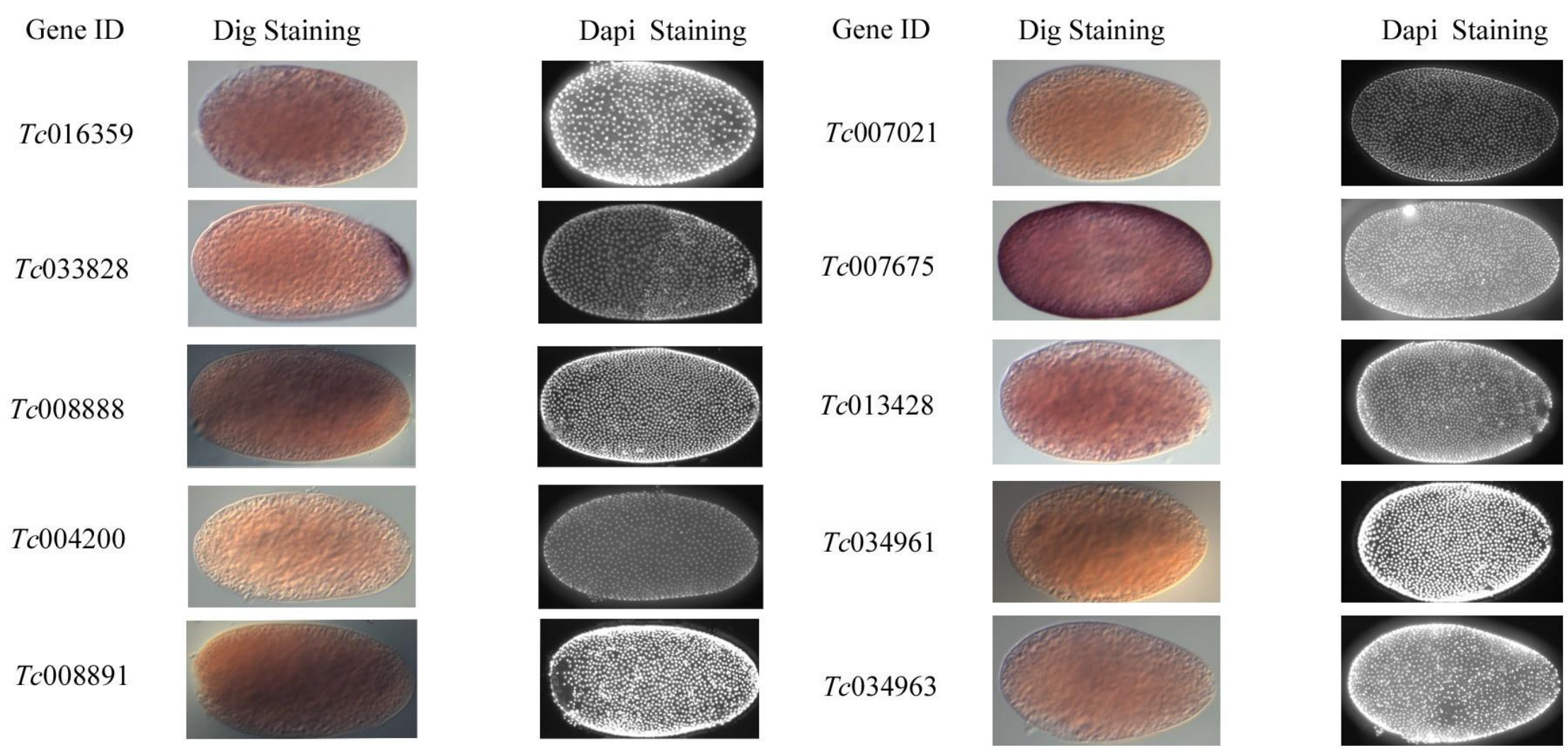

Fig 3a: 10 selected genes from the Tissue/stage specific embryonic transcriptomic data 

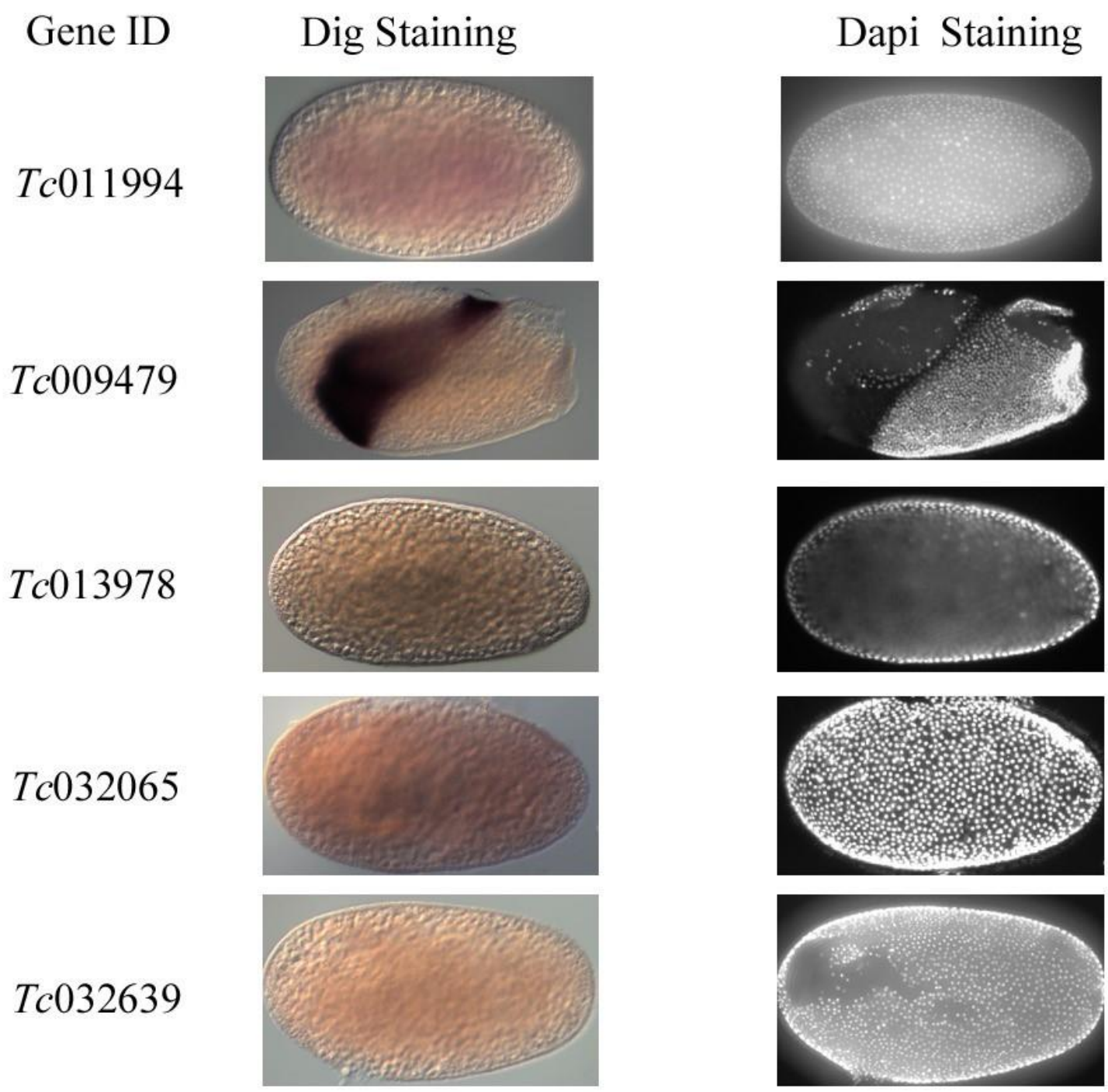

Fig 3b: 5 selected genes from the systematic reverse genetics data 

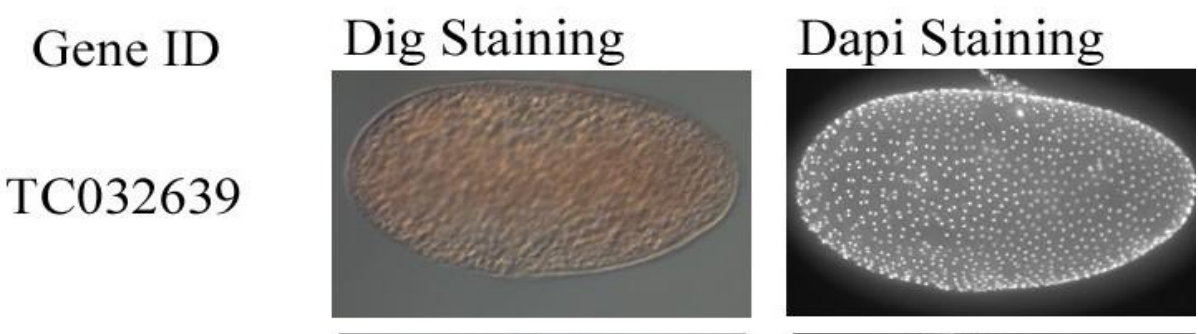

TC032065
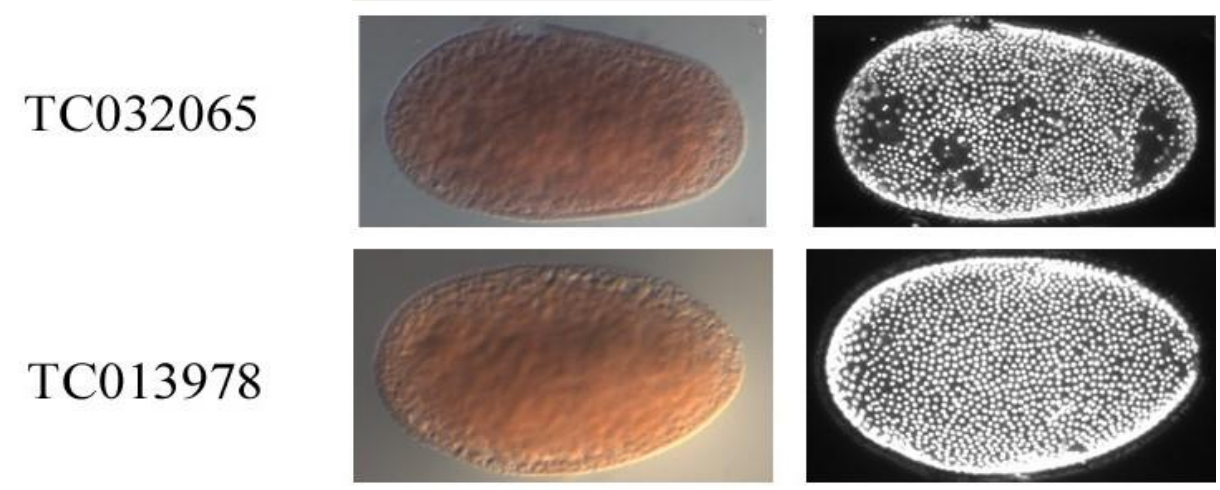

TC007675
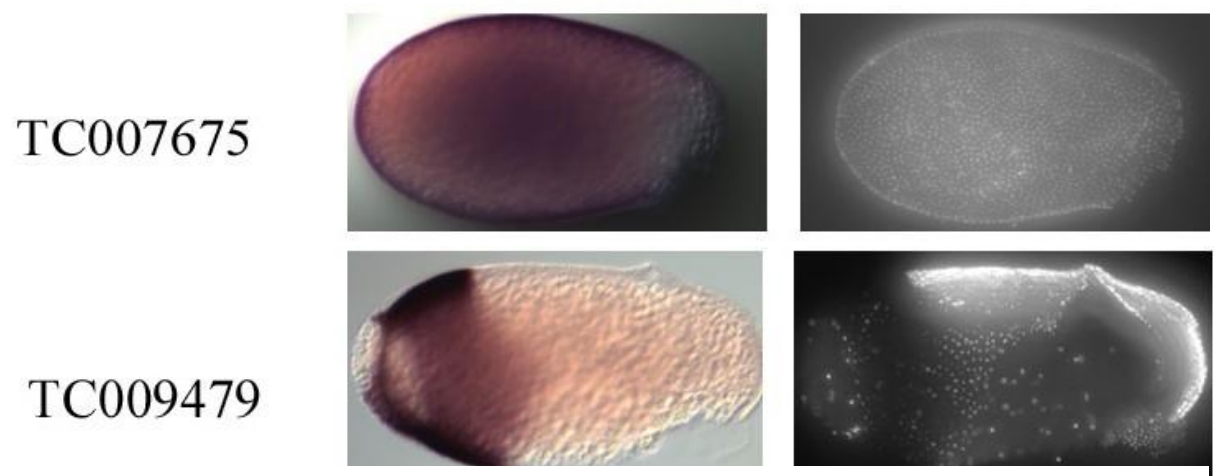

TC013428
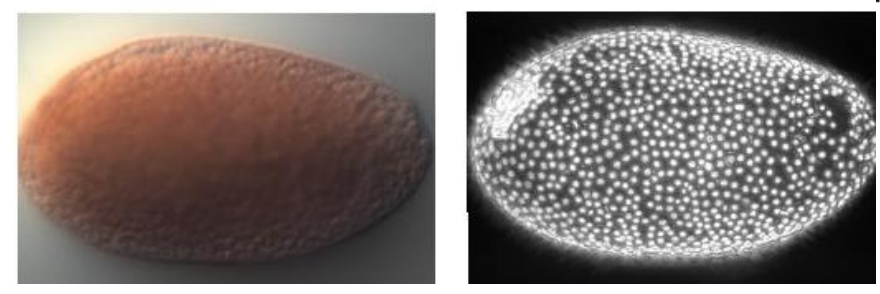

TC011994
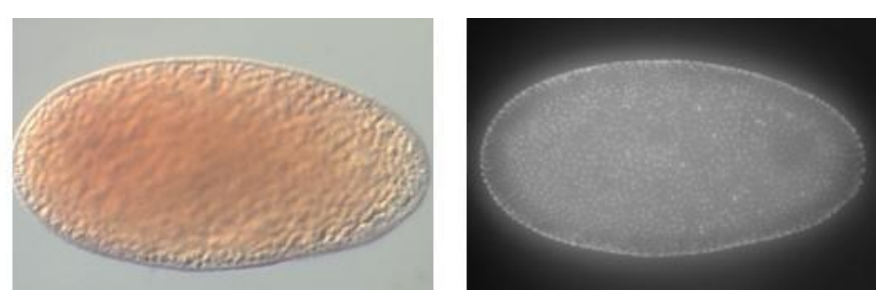

Fig 4: Whole mount In-Situ hybridization showing expression patterns of 7 potential cellularization specific expression based on a probe based on the full transcript 


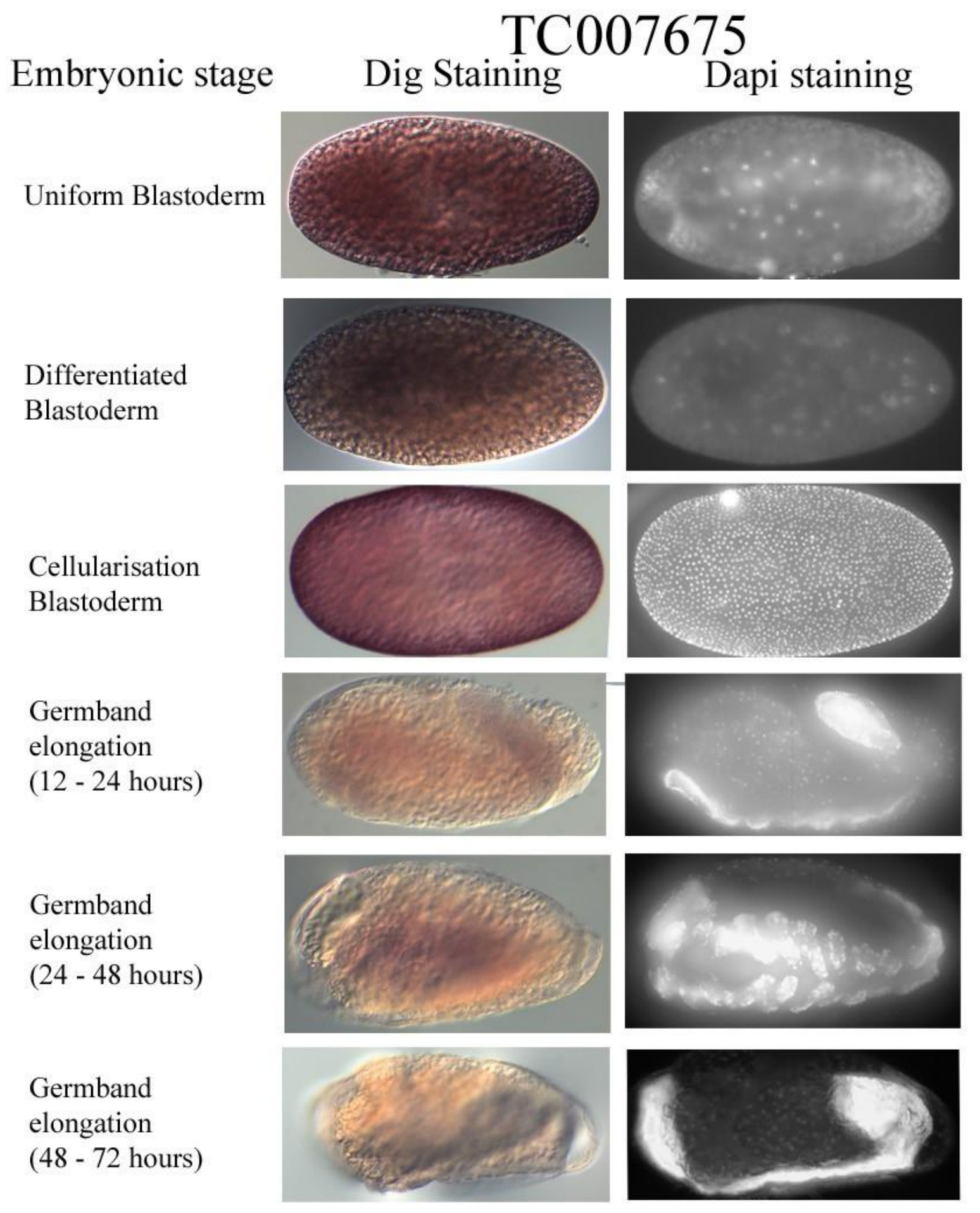

Fig 5: Whole mount In-Situ hybridization showing expression pattern of Tc007675 gene in embryos in a stage (time) specific manner. 


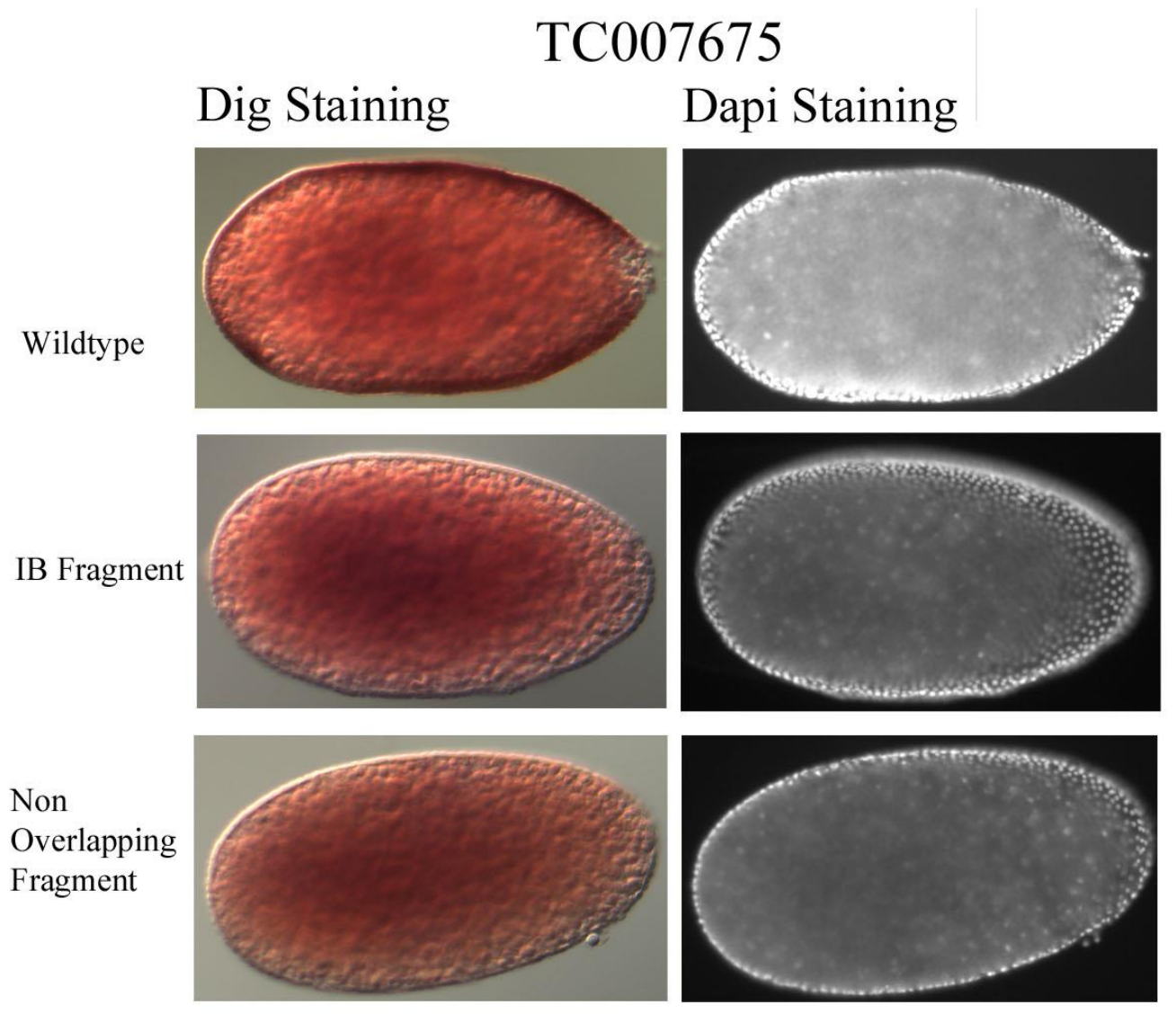

Fig 6: Expression pattern of iB-07126 and iB-07126_2 (TC007675) by whole-mount in-situ hybridization on 0-24 hours old San Barnadino wildtype embryos 


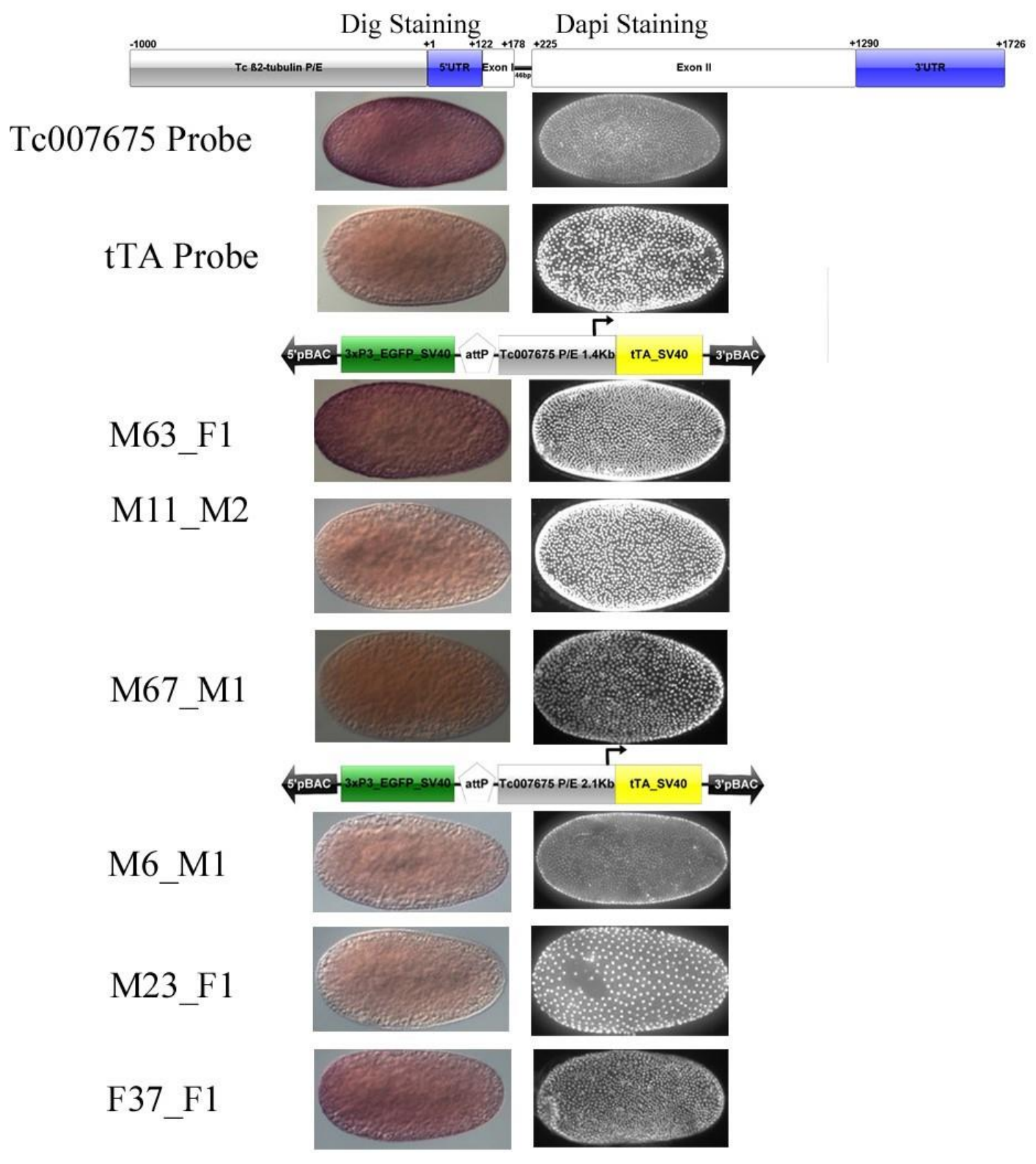

Fig 7: Whole Mount In-Situ Hybridization of wildtype, transgenic $1.4 \mathrm{~Kb}$ and $2.1 \mathrm{~Kb}$ strains embryos against Tc007675 and tTA antisense probes. 
A)

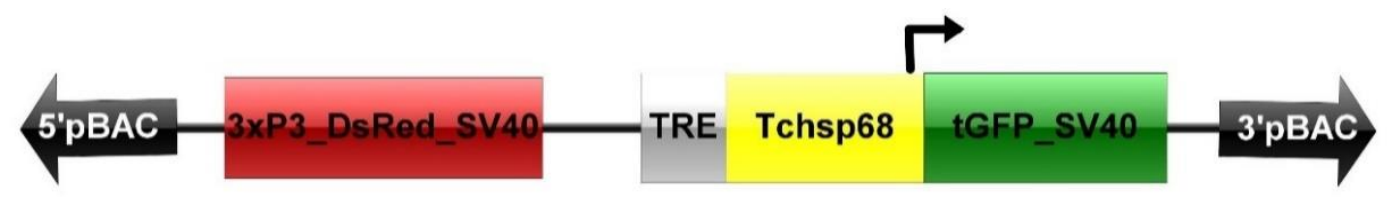

B)

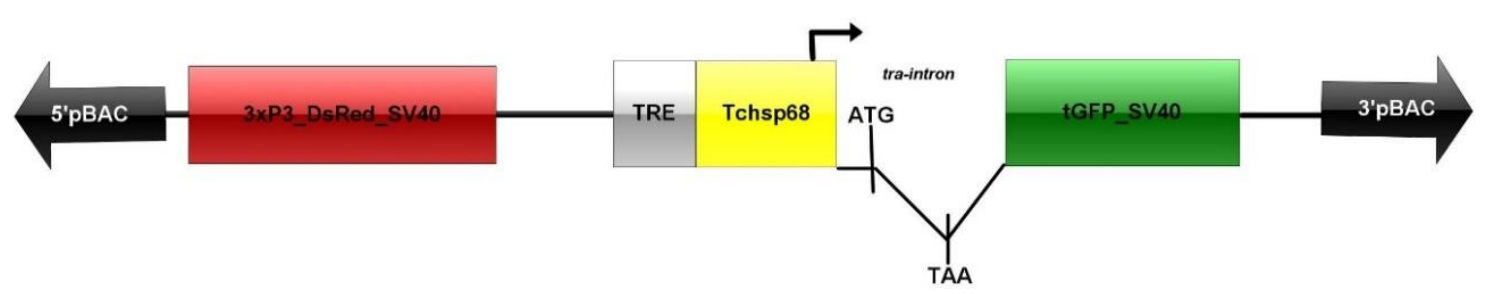

Fig 8: piggyBac-based transgenic reporter construct (A) without Tc intron and (B) with Tc-tra intron clone for sexing during early embryonic development. 


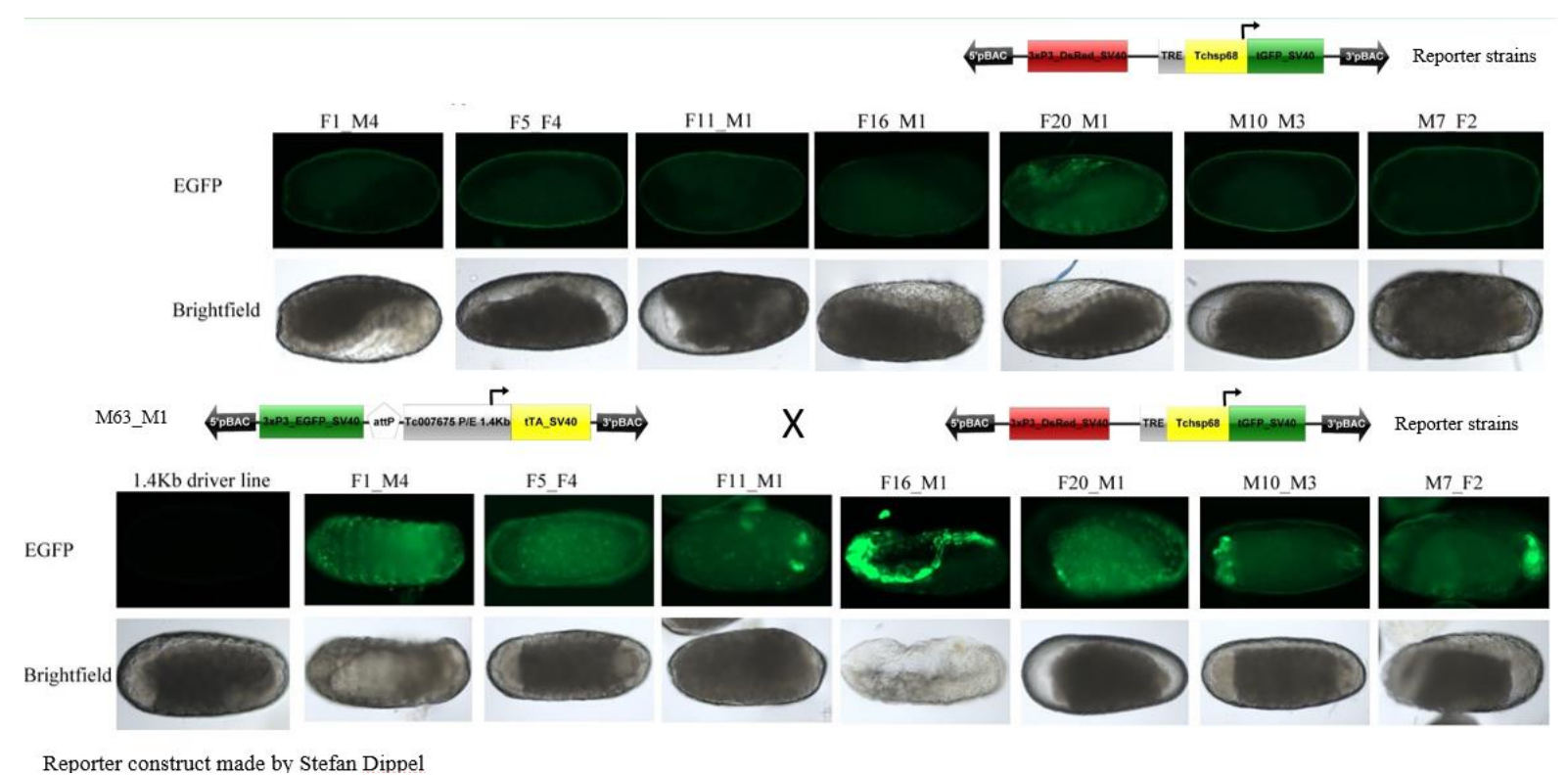

Fig 9a: Embryos from M63_F1 driver strain and several reporter strains showing varying degrees of EGFP expresssion 

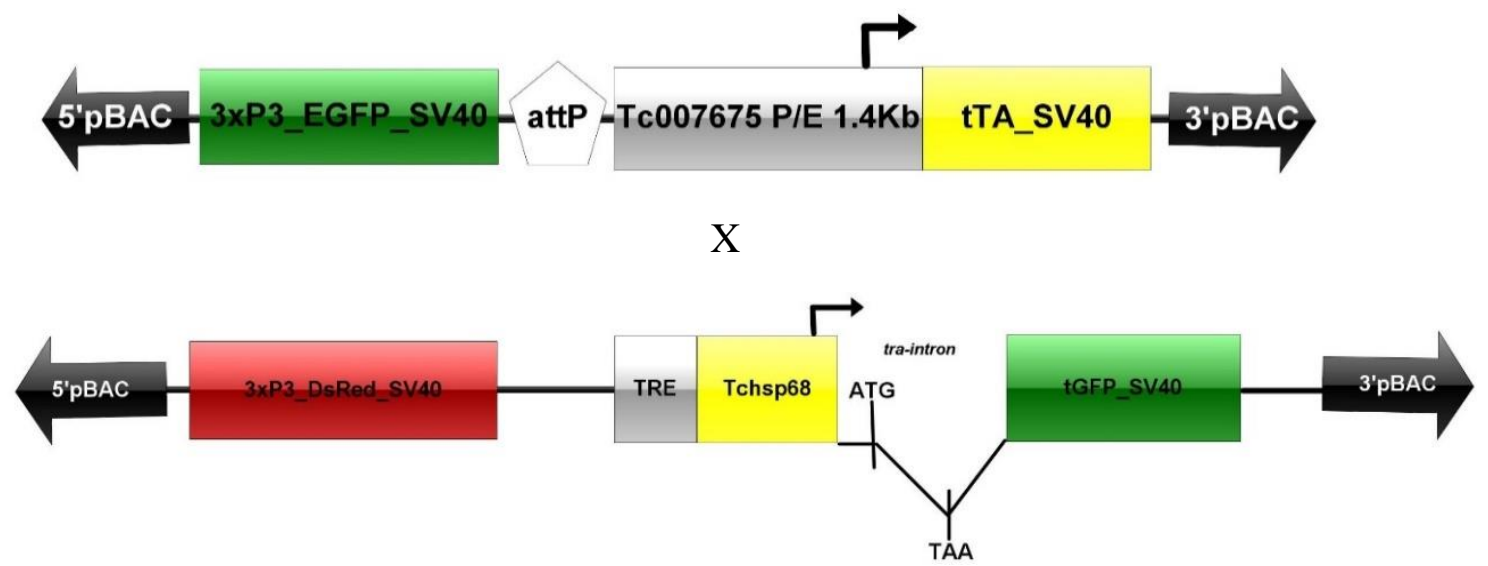

\section{Brightfield EGFP Channel DsRed Channel}

Wildtype
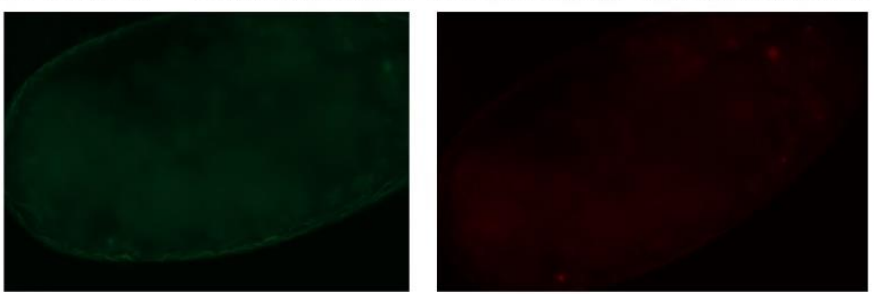

F35 F1
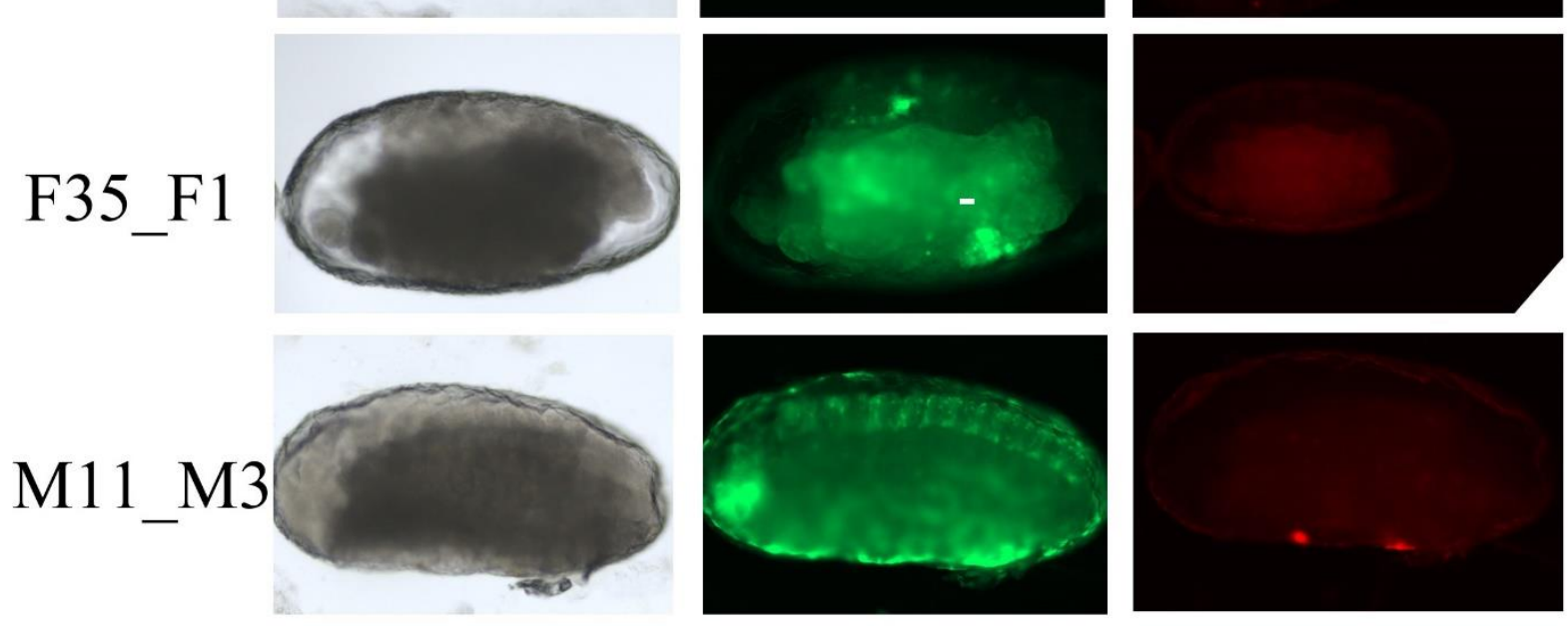

Fig 9b: Embryos from M63_F1 driver strain and several sexing strains strong EGFP expresssions. 
Table 1: comprehensive list of the selected genes highlighting some of their properties and characterization

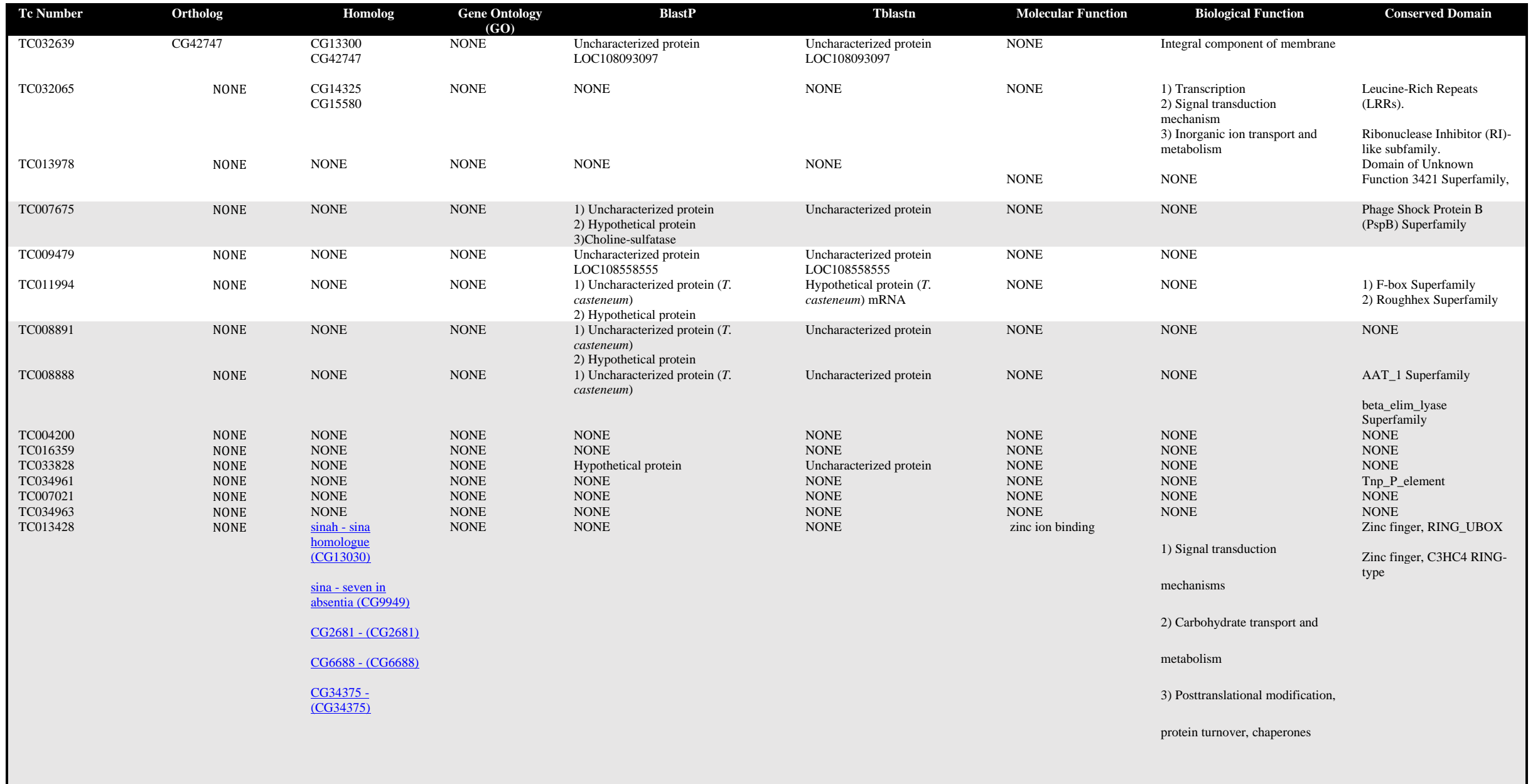

NB: Gray coloured rows are the genes obtained from tissue/stage specific embryonic transcriptomic data while White rows are from systematic reverse genetics empty egg phenotypes iBeetle data. 
Table 2:

\begin{tabular}{|l|l|l|}
\hline IB Number & Fragment & Sequence \\
\hline iB_07126 & iB Fragment & AGGACCGTTGTGACACCATTGACGAGTTATGTCGGGATTATTTAAGTCTTGATGA \\
& & GGGATTTTAACCAGGAGCTCATCTCCAGAAGAATTGGCTTTGACACCGACTTTT \\
& & GAGGAAGATATGTTAGTTTCCGAAAGTTTGGTGCAATTTTGTGCAGAAAACTGTG \\
& & GAGCAGCGAAATAGTGAAAAAAGTGTGTGGAAAAAATGCGCAAAGTGGAGAGAC \\
& & TGTTTGGAATTTTTTCTGCCCGAAAATCATCGACGGCCAATTGCAAACCACAGTG \\
& & TGATAAATAATGTAATAAATGGAACTGTTTGACGGAGA \\
\hline iB_07126_2 & Non over lapping & CGACCTGAAGTCTCCACTCCCCCCGTCATCGCTTCGGCAAAAAAACACGCAATGT \\
& & CCACCCCCAAAACCAACCCGAAAAAAACACCCGACATGACCCAGTTACTGGAACA \\
& & GTTGGAACAGACAGGAAACG \\
\hline
\end{tabular}

Table 3: RNAi experiment using Tc007675 dsRNA (iBeetle fragment and NOF) and DsRed (control) showing the number of San Barnadino wildtype female pupae injected and survival percentages.

Number of Injected $\quad \mu \mathrm{g} / \mu \mathrm{l} \quad$ Number of female pupae $\quad$ Number of dead female pupae $\quad \%$ suvival

RNAi titre

Female pupae

survived to adults

\begin{tabular}{llllll}
\hline iB_07126 (OiB) & 21 & 1 & 20 & 1 & 95.24 \\
iB_07126_2 (NOF) & 24 & 1 & 22 & 2 & 91.67 \\
DsRed (Control) & 25 & 1 & 23 & 2 & 92 \\
\hline
\end{tabular}


Supplementary Table 1: Primers name used for amplification, RACE and final amplification of gene fragments after RACE

\begin{tabular}{|l|l|l|l|l|l|l|}
\hline $\begin{array}{l}\text { Te } \\
\text { number }\end{array}$ & $\begin{array}{l}\text { Forward } \\
\text { primer } \\
\text { code }\end{array}$ & $\begin{array}{l}\text { Reverse } \\
\text { primer } \\
\text { code }\end{array}$ & $\begin{array}{l}\text { 5'RACE } \\
\text { primer } \\
\text { code }\end{array}$ & $\begin{array}{l}\text { 3'RACE } \\
\text { primer } \\
\text { code }\end{array}$ & $\begin{array}{l}\text { 5'Forward } \\
\text { primer } \\
\text { code }\end{array}$ & $\begin{array}{l}\text { 3'Reverse } \\
\text { primer } \\
\text { code }\end{array}$ \\
\hline TC034963 & MID\#73 & MID\#72 & & & & \\
\hline TC007021 & MID\#68 & MID\#69 & & & & \\
\hline TC016359 & MID\#58 & MID\#59 & & & & \\
\hline TC034961 & MID\#62 & MID\#63 & & & & \\
\hline TC033828 & MID\#36 & MID\#37 & & & & \\
\hline TC008888 & MID\#28 & MID\#29 & & & & \\
\hline TC008891 & MID\#70 & MID\#71 & & & & \\
\hline TC004200 & MID\#32 & MID\#33 & & & & \\
\hline TC007675 & MID\#34 & MID\#35 & MID\#93 & MID\#94 & MID\#111 & MID\#112 \\
\hline TC011994 & MID\#40 & MID\#41 & MID\#95 & MID\#96 & MID\#113 & MID\#114 \\
\hline TC032065 & MID\#52 & MID\#53 & MID\#89 & MID\#90 & MID\#109 & MID\#110 \\
\hline TC032639 & MID\#54 & MID\#55 & MID\#91 & MID\#92 & MID\#107 & MID\#108 \\
\hline TC013978 & MID\#56 & MID\#57 & MID\#99 & MID\#100 & MID\#115 & MID\#116 \\
\hline TC009479 & MID\#60 & MID\#61 & MID\#101 & MID\#102 & MID\#117 & MID\#11 \\
\hline TC013428 & MID\#64 & MID\#65 & MID\#97 & MID\#98 & MID\#119 & MID\#120 \\
\hline
\end{tabular}


Supplementary Table 2: List of primers used in this experiment

\begin{tabular}{|c|c|c|}
\hline ID & NAME & Sequence 5'-3' \\
\hline MID\#1 & Tc-TcTraIn_hsp68 F & gtaccggtcgccaccatggtaattggttttcatagtcttgacgaa \\
\hline MID\#2 & TcTra_tGFPO R & cgctctcgtcgetctccatctgaaatcaagcggttctct \\
\hline MID\#28 & TC032639_F & TGTGAGCAATCGTGTCGGAA \\
\hline MID\#29 & TC032639_R & GACGTGCTATGTGCTATGATAATTTGT \\
\hline MID\#32 & TC013978_F & TGGCTTGGAGAATTCGGCAT \\
\hline MID\#33 & TC013978_R & CCAGGATGGGCATAATCTGA \\
\hline MID\#34 & TC007675_F & ACAACAATAATGTTCTCGAGAGC \\
\hline MID\#35 & TC007675_F & GCAAACCACAGTGTGATAAATAATGTA \\
\hline MID\#36 & TC009479_F & ATCATGAAGTGGTTTCTCTTAATATTTG \\
\hline MID\#37 & TC009479_R & TGCGCTGCATACACACCTAA \\
\hline MID\#40 & TC011994_F & ATGTATCAAGTGCTTCCATTCCTC \\
\hline MID\#41 & TC011994_R & GACCTTGTAAAATGTTAAGACTGTAAAT \\
\hline MID\#52 & ECG-TC_008891_F & AGAAATAGTTTTTGGAATTACGTCC \\
\hline MID\#53 & ECG-TC_008891_R & ATATCTTCCGTAATATGGGTAGTATG \\
\hline MID\#54 & ECG-TC_008888_F & ATGTACACCAAACTTTTCGCTCTCTTC \\
\hline MID\#55 & ECG-TC_008888_R & CTATTTTACAAGCTTGATGGGATTAAC \\
\hline MID\#56 & ECG-TC_004200_F & ATCTGCATATTTGGACCAAATTG \\
\hline MID\#57 & ECG-TC_004200_R & TTCCGCGTATGTGACAATATGGTG \\
\hline MID\#58 & ECG-TC_016359_F & ATGGATCCTCAAGCCTCAAGTG \\
\hline MID\#59 & ECG-TC_016359_R & TTACGACTGCAACCAAAAATTAAATC \\
\hline MID\#60 & ECG-TC_033828_F & ATGCGCTTATTCCTTAACGAACAGC \\
\hline MID\#61 & ECG-TC_033828_R & TTACAGATAATATTTATTTTCAGTGCGTGC \\
\hline MID\#62 & ECG-TC_034961_F & ATGTTGCGAGGTTTATTTTCAAAC \\
\hline MID\#63 & ECG-TC_034961_R & AGGCGTATTGTTTTCTTCAACGTTTC \\
\hline MID\#64 & ECG-TC_007021_F & ATGGGACGGCTACAGGTTTTG \\
\hline MID\#65 & ECG-TC_007021_R & TCATATGACAACCCGACTGAAC \\
\hline MID\#68 & ECG-TC_013428_F & ACTGCTCGAAATGCGACAAC \\
\hline MID\#69 & ECG-TC_013428_R & TCAAGATCAATCTGAAGCTTCTCCTTATC \\
\hline MID\#70 & TC-032065_F2N & ATGCGCGTCCCTTACAAAATC \\
\hline MID\#71 & TC-032065_R2N & TTAAGCCATGCATGAGGACAC \\
\hline MID\#72 & TC-034963_F2N & GTGACACTTTAAGCCGATGGTAC \\
\hline MID\#73 & TC-034963_R2N & AGGAGTACTTAACCGGAATTGAGTG \\
\hline MID\#89 & TC008891_5' & CAGACGAGGTGCACATATCTTCCGT \\
\hline MID\#90 & TC008891_3' & TCCTAATTGTTTTGGCCGCTTTAATGG \\
\hline MID\#91 & TC008888_5' & CAAGCTTGATGGGATTAACGACGCTG \\
\hline MID\#92 & TC008888_3' & ACTTTTCGCTCTCTTCGCCCTCTTG \\
\hline MID\#93 & TC007675-5' & CCAAACAGTCTCTCCACTTTTGCGCA \\
\hline MID\#94 & TC007675-3' & GATTCGATTACTACCCCCGACCTGAA \\
\hline MID\#95 & TC011994-5' & AAGGTCTCTCTTCTTCCTCTGGCCGTCCT \\
\hline MID\#96 & TC011994-3' & GTTCAGGAAGAATTGATTGCCGAACG \\
\hline MID\#97 & TC007021_5' & CCCGACTGAACTTTATGGTTTTTCTTCC \\
\hline MID\#98 & TC007021_3' & ACAAGAACAACCGACAGCCCCCGCAC \\
\hline MID\#99 & TC004200-5' & GAGAAGCTTGCAGACCGTGGCGCCATC \\
\hline MID\#100 & TC004200-3' & GTGACCTGAGAAATAGTGATCAGCTTGC \\
\hline MID\#101 & TC033828-5' & GTTCTTGCGGTAGTCTTGTTGCGG \\
\hline MID\#102 & TC033828-3' & TGGGAGGTTTATCGACAATGTGAAGTTC \\
\hline MID\#103 & ClaI-EGFP-SV40_F2 & GAGGATCGATATGGTGAGCAAGGGCGAG \\
\hline MID\#104 & HindIII-EGFP-SV40_R & TTTAAGCTTGATGAGTTTGGACAAACCACAAC \\
\hline MID\#108 & TC008888R-NEW-P & CCCTTAATTTTTTATTTAAAACACCC \\
\hline MID\#109 & TC008891F-NEW-P & CAGTTCTTAGAACACGAAAAACAACC \\
\hline MID\#110 & TC008891R-NEW-P & CATGCTACAACTGCTTTATTTCG \\
\hline MID\#111 & TC007675F-NEW-P & CACTTGTTGTTTAAACCCCACAAC \\
\hline MID\#112 & TC007675R-NEW-P & TCAAAATCATTTATTTACATGCAGTCTTC \\
\hline MID\#113 & TC011994F-NEW-P & GCTTCCCAAGATGTATCAAGTGC \\
\hline MID\#114 & TC011994R-NEW-P & CCCTTGCTTCCCAAGATGTATC \\
\hline MID\#117 & TC007675-F-1.4Kb-Nco1 & GAGGCCATGGGTGCTGCATTTCATGACTTC \\
\hline MID\#118 & TC007675-R-1.4Kb-Cla1 & GCCGATCGATTATTGTTGTTGGGTTTAAACAACAAGTG \\
\hline MID\#120 & TC007675-R-2.1Kb-Xba1 & AATCTAGATATTGTTGTGGGGTTTAAACAACAAGTG \\
\hline MID\#123 & TC007675P/E-N-Nco1F & ATGGCCATGGGCAAGTCAGGAAGAAATTTCAAGTTT \\
\hline No.5 & iPCR5'F1 & GACGCATGATTATCTTTTACGTGAC 1st round PCR \\
\hline No.6 & iPCR5'R1 & TGACACTTACCGCATTGACA \\
\hline No:7 & iPCR5'F2 & GCGATGACGAGCTTGTTGGTG 2nd round PCR \\
\hline No.8 & iPCR5'R2 & TCCAAGCGGCGACTGAGATG \\
\hline No.9 & iPCR5'Seq & CGCGCTATTTAGAAAGAGAGAG \\
\hline MFS227 & & AGTCAGTCAGAAACAACTTTGGCACATATC \\
\hline \multirow[t]{6}{*}{ MFS228 } & & CCTCGATATACAGACCGATAAAACACATGC \\
\hline & Pjet 1.2 Forward & CGACTCACTATAGGGAGAGCGGC \\
\hline & Pjet 1.2 Reverse & AAGAACATCGATTTTCCATGGCAG \\
\hline & Pjet 1.2 Forward_T7 & GAATTGTAATACGACTCACTATAGGCGA CTCACTATAGGGAGAGC \\
\hline & M13-Forward & GTAAAACGACGGCCAGTG \\
\hline & M13-Reverse & CGAGAAACAGCTATCACC \\
\hline
\end{tabular}


Supplementary Table 3: Annealing temperature for the amplification of 15 genes cellularization specific genes fragments

\begin{tabular}{|c|c|c|}
\hline Primer ID & Gene ID & $\begin{array}{l}\text { Annealing temperature for } \\
\text { PCR reaction }\end{array}$ \\
\hline MID\#28 & \multirow{2}{*}{ TC032639 } & \multirow{2}{*}{65} \\
\hline MID\#29 & & \\
\hline MID\#70 & \multirow[t]{2}{*}{ TC032065 } & \multirow[t]{2}{*}{63} \\
\hline MID\#71 & & \\
\hline MID\#32 & \multirow[t]{2}{*}{ TC013978 } & \multirow[t]{2}{*}{62} \\
\hline MID\#33 & & \\
\hline MID\#34 & \multirow[t]{2}{*}{ TC007675 } & \multirow[t]{2}{*}{62} \\
\hline MID\#35 & & \\
\hline MID\#36 & \multirow[t]{2}{*}{ TC009479 } & \multirow[t]{2}{*}{61} \\
\hline MID\#37 & & \\
\hline MID\#40 & \multirow[t]{2}{*}{ TC011994 } & \multirow[t]{2}{*}{61} \\
\hline MID\#41 & & \\
\hline MID\#52 & \multirow[t]{2}{*}{ TC008891 } & \multirow[t]{2}{*}{61} \\
\hline MID\#53 & & \\
\hline MID\#54 & \multirow[t]{2}{*}{ TC008888 } & \multirow[t]{2}{*}{61} \\
\hline MID\#55 & & \\
\hline MID\#56 & \multirow[t]{2}{*}{ TC004200 } & \multirow[t]{2}{*}{61} \\
\hline MID\#57 & & \\
\hline MID\#58 & \multirow[t]{2}{*}{ TC016359 } & \multirow[t]{2}{*}{62} \\
\hline MID\#59 & & \\
\hline MID\#60 & \multirow[t]{2}{*}{ TC033828 } & \multirow[t]{2}{*}{64} \\
\hline MID\#61 & & \\
\hline MID\#62 & \multirow[t]{2}{*}{ TC034961 } & \multirow[t]{2}{*}{62} \\
\hline MID\#63 & & \\
\hline MID\#64 & \multirow[t]{2}{*}{ TC007021 } & \multirow[t]{2}{*}{63} \\
\hline MID\#65 & & \\
\hline MID\#72 & \multirow[t]{2}{*}{ TC034963 } & \multirow[t]{2}{*}{64} \\
\hline MID\#73 & & \\
\hline MID\#68 & \multirow[t]{2}{*}{ TC013428 } & \multirow[t]{2}{*}{64} \\
\hline MID\#69 & & \\
\hline
\end{tabular}


3.3 Novel genetic engineering approach for the management of coleopteran insect pests using CRISPR-Cas9.

In this chapter we intend to mimic the effect of radiation by affecting reproductive sterility but by using the spermatogenesis specific $\beta 2 t$ gene to generate driver strains which can express tTA in the testis of the males. Using the tet-off system we want to conditionally drive Cas9 protein in the testis to cause shredding of the sperm chromosomes at random locations by providing multiplex of gRNAs targeting several repetitive genomic loci.

ISAH, Musa Dan'azumi \& Ernst A. Wimmer

Authors contribution to the practical work

ISAH, Musa Dan'azumi: Conceived and design the experiment, conducted all the other experiment and analysis. Wrote the manuscript.

\section{Status: Work in progress}




\title{
Novel genetic engineering approach for the management of coleopteran insect pests using CRISPR-Cas9.
}

ISAH, Musa Dan'azumi \& Ernst A. Wimmer

1) Department of Developmental Biology, Johann-Friedrich-Blumenbach-Institute of Zoology and Anthropology, Göttingen Center for Molecular Biosciences, Georg-August-University Göttingen, 37077 Göttingen, Germany.

2) Department of Crop Science, Faculty of Agriculture, Wildlife and Forestry Resources Management, University of Calabar, P.M. B. 1152, Calabar, Cross River State, Nigeria.

* Corresponding Author: E. A. Wimmer. Tel. +49 5513928666

E-Mail address: ewimmer@gwdg.de.

\begin{abstract}
:
Coleoptera, also known as beetles, constitute about the quarter of all the described animal sp in the world, and they are found occupying all ecological niches. There are approximately about 380, 000 species of coleoptera that have been taxonomically characterized and with many more still to be discovered. The Red Flour Beetle (Tribolium casteneum) also known as darkling beetle has been developed into a laboratory model organism due to its ease of rearing, high fecundity and also full genomic sequence, which make it a suitable organism aside from Drosophila (Order: Diptera) for various genomic studies. It is therefore helping in answering several developmental and evolutionary biology questions. Using a Tribolium sperm specific spermatogenesis gene $(T c-\beta 2 t)$, several transgenic driver strains were generated by fusing the amplified $1 \mathrm{~Kb}$ Promoter/Enhancer (P/E) of $T c-\beta 2 \mathrm{t}$ to the gene encoding tTA. Results of Whole Mount in Situ Hybridization (WMISH) of generated transgenic line testes against tTA antisense probe showed that only one of the four transgenic lines has a clear expression pattern (F5_F1) in the testis that resemble the wildtype endogenous expression pattern. In addition, responder line expressing Cas 9 driven by Tetracycline Responder Element (TRE), and $T$. castaneum heat shock basal promoter (Tchsp68) was generated. However, When the driver strains were crossed to the potential Cas9 expressing effector strain in a tet-off binary expression system, no specific gene expression pattern was observed in WMISH against Cas9 in their testes. 710757
\end{abstract}

Keywords: Coleoptera, Red Flour Beetle, Cas9, binary expression system, gene expression 


\section{Introduction:}

The Sterile Insect Technique (SIT) is a birth management practice that is utilized for the management of pest population ${ }^{1}$. SIT is target-specific and environment-friendly ${ }^{5,6}$ approach, and its application reduce possible pesticide use. It employs rearing and large release of reproductively sterile male, in order to search for and mate with conspecific individual, which will subsequently result in infertile mating of wildtype females ${ }^{2}$. Centralized to SIT strategy relies is the releases of radiated ${ }^{3}$ or chemo-sterilized insects male individuals. Thus, between the two approaches of exposure of the insects for sterilization, ionizing radiation ${ }^{3}$ such as the use of Cobalt-60 or Caesium-137 have been adopted for the control of many insect species even though at the inception of SIT programs chemo-sterilant and radiation were simultaneously used for the sterilization of insects with their efficiencies reported to be the same ${ }^{4}$. The adoption of radiation as the method of choice ultimately is because chemosterilants that are used are found to be carcinogenic, teratogenic, and mutagenic ${ }^{14}$. The Sterilization process thus guarantees the sterility of the released insect, and an insect is termed sterile if it cannot produce viable offspring as a result of the treatment ${ }^{7}$ applied.

Transgenic technology is the insertion of desired gene sequences into another clade, or modified and introduced into the same clade for the expression of a desired trait ${ }^{8}$. Transgenesis has provided an efficient tool, and as an alternative to the issues caused by ionizing radiation genetic systems. Also, using a repressive system by adding food with tetracycline (Figure 1) ${ }^{9}$ or other food additives ${ }^{7}$ supplement, a conditional system can be generated for establishment of sexing and sterility system based on binary expression system.

The tetracycline-controlled transactivator (tTA) system initiate the gene expression of the responder elements by binding to a tTA-Response Element (TRE) ${ }^{10}$ that is placed downstream of the responder element. The best-known tetracycline-regulated systems ${ }^{11}$ is functional by addition of tetracycline in the food i.e. when tetracycline is not supplemented in the food, the synthetic transactivator (tTA) is activated and gene expression is obtained because of the binding of the tTA to the TRE. However, when tetracycline is added to the food, the system is shut down and no expression of target gene will be observed due to the conformational change that tTA undergoes, and also because it's being blocked by the tetracycline ${ }^{1112}$ supplemented in the diet. The tet off and tet on systems have both been used across a very wide of insect pests such as in Drosophila ${ }^{13}$ to mediate transgenic sexing and sterility. This system has also been transferred to the Tephritid pest species Ceratitis capitata ${ }^{16}$ and Anastrepha suspensa ${ }^{17}$ by 
isolating species-specific blastoderm-expressed genes $18 \quad 19$ and their respective promoters/enhancers region for the development of sexing system and other biotechnological approaches. The possibility that the system can be switched off and on with tetracycline (Figure 1) ${ }^{9}$, thereby making it the most favorable expression system in developing transgenic SIT approaches. Thus, the system can also be used to induce reproductive sterility ${ }^{14}$, where sterility is caused by a combination of transgenes ${ }^{15}$ in a binary system.

Thus, due to the suitability and applicability of this system in sexing ${ }^{17,20,21}$ and female-specific embryonic lethality systems (FSEL) ${ }^{16}$ in other dipteran insects, we want to employ the system in Tribolium castaneum using CRISPR/Cas9 ${ }^{22} 23$ system. Strain expressing Cas 9 is to be used as effector line driven by TRE and a multiplex of gRNAs targeting multiple genomic loci such as para-centrometric, sub-telomeric and microsatellites ${ }^{24} 25$ to induce sterility via Double Strand Breaks (DSBs) ${ }^{26}$ which will ultimately imitate the effect of radiation due to aneuploidy. The expression of CRISPR/Cas9 in responder lines driven by tTA clone to the Tc- $32 \mathrm{t}$ enhancer/promoter as previously proposed ${ }^{9}$ (Figure 2) is targeted during spermatogenesis.

Thus, based on the reliability and functionality of the system for gene expression, we thus intend to utilize the system during spermatogenesis to generate and establish a reproductive sterility system in $T$. castaneum the model for coleopteran insect pests. 


\section{RESULTS:}

\section{Generation of spermatogenesis specific driver lines.}

To generate transgenic strains that will specifically express tetracycline trans-activator (tTA) during spermatogenesis, which will subsequently activate the expression of Cas 9 protein for a CRISPR/Cas9 mediated chromosome shredding to induce sterility due to aneuploidy. We generated several transgenic lines by injecting fused $1 \mathrm{~Kb}$ Promoter/Enhancer $(\mathrm{P} / \mathrm{E}) \mathrm{Tc}-\beta 2 \mathrm{t}$ to gene encoding tTA (Fig; 3a) by piggyBac based mediated germline transformation. We identified 7 transgenic lines i.e.F5_F1, F5_F2, F5_M1, F10_F1, F10_F2, F10_F3 and F10_M1 from $2 \mathrm{G}_{0}$ (F5 and F10) individuals, by their mediated green-eyed fluorescence transformation marker.

\section{Generation of responder lines usable for conditional chromosome shredding.}

To generate responder lines that will express Cas 9 controlled by Tetracycline Responder Element (TRE), and T. castaneum basal promoter (Tchsp68). A construct was generated by substituting Dmhsp43 from already existing plasmid (gift from Ahmed) with Tchsp68.

Using piggyBac mediated germline transformation, i obtained one potential Cas9 expessing effector transgenic line F8_F1, by injecting the transformation vector pBac \{a_3xP3_DsRed_SV40_attP_TRE_hsp68_Cas9_SV40\} (Fig; 4) construct.

\section{Testing of binary expression system during spermatogenesis.}

The functionality of the driver lines was verified by the detection of the expression pattern of tTA in testis of the generated transgenic lines by in situ hybridization (Fig: 3b). For each independent $\mathrm{G}_{0}$ (F5 and F10) mothers 2 independent $\mathrm{F}_{1}$ progenies were selected, their testis dissected ${ }^{27}$ and tested with WMISH. The result of the WMISH showed that the line F5_F2 (Fig: 3b) testes shows an observable pattern which resembles the endogenous expression. To further validate the ability of the tTA driver line ability to mediate expression of Cas 9 in the sperms, i crossed the tTA driver strains F5_F1, F5_F2, F10_F3 and F10_M1 (Fig: 3) to the TRE_Cas9 responder strain F8_F1 (Fig: 4). WMISH, using Cas9 probes could not detect Ca9 expression driven by tTA in the testis for any of the driver line (Fig 4) for now. 


\section{MATERIALS AND METHOD:}

\section{Beetle stock and rearing:}

Red Flour Beetle (RFB), Tribolium castaneum vermillion ${ }^{\text {white }}\left(v^{w}\right)$ strain was used in all experiments. Beetle stocks were fed on full grain flour supplemented with $5 \%$ yeast powder in 4.5liter square plastic boxes $(15 \mathrm{~cm} \times 15 \mathrm{~cm} \times 20 \mathrm{~cm})$ with ventilation on the lid and were kept at $32^{\circ} \mathrm{C}$ with constant light ${ }^{25}$.

\section{RNA isolation:}

Total RNA was extracted using ZR Tissue \& Insect RNA MicroPrep (Zymo Research Europe, 79110 Freiburg). The extracted RNA was treated with TURBO ${ }^{\mathrm{TM}}$ DNase in column digestion to remove any residual DNA contamination. TURBO ${ }^{\mathrm{TM}}$ DNase was inactivated using the DNase inactivation reagent as described in TURBO DNA-free ${ }^{\mathrm{TM}}$ kit Ambion; AM1907' manual. The RNA concentration was measured using NanoDrop 1000 Spectrophotometer (Thermo Scientific ${ }^{\mathrm{TM}}$, USA) and kept in $-80^{\circ} \mathrm{C}$ freezer until use.

\section{cDNA synthesis:}

After RNA isolation and measurement, $1 \mu \mathrm{g}$ of the RNA was used for the first strand cDNA synthesis using Invitrogen ${ }^{\mathrm{TM}}$ SuperScript $^{\mathrm{TM}}$ Reverse Transcriptase (Thermo Scientific ${ }^{\mathrm{TM}}$, USA). Generated cDNA was kept at $-80^{\circ} \mathrm{C}$ and used later as template for the amplification of specific and selected gene sequences by PCR.

\section{Generation of B2-tubulin probe}

We generated an RNA antisense probe for Tc-B2-tubilin that was used for WMISH. We make the probe from a generated template and used for in vitro transcription, by first conducting a PCR amplification of about 800bp from the generated cDNA using MID\#203/ MID\#204 primers. The PCR amplification protocol include initial denaturation at $98^{\circ} \mathrm{C}$ for 3 minutes, followed by 35 cycles of denaturation at $98^{\circ} \mathrm{C}$ for $30 \mathrm{sec}$., $72^{\circ} \mathrm{C}$ for 1 minute and final elongation step of 10 minutes. The amplified fragment was run on 1\% agarose gel and distinct single band was excised and purified. The purified fragment was ligated into pJet 1.2 vector. After transformation into DH5 competent cells, antisense positive clones were identified by cloning PCR using MID\#203 and pJet 1.2 forward primer. 
Antisense RNA probe was synthesized using DIG-labelling kit (Thermo Fisher Scientific) by using $500 \mathrm{ng}$ of DNA as template in a total reaction mix of $10 \mu \mathrm{l}$. The probe was precipitate and resuspended in $100 \mu 1$ RNA resuspension buffer.

Testis of adult transgenic males were dissected in a puddle of insect saline solution and fixed in farmers fixative ${ }^{27}$ solution for 5 minutes afterwards it was used as a tissue for WMISH.

\section{Generation of spermatogenesis specific driver construct.}

The first cloning step for the generation of pBac \{a_3xP3_EGFP_SV40 _attP_B2t_tTA_SV40\} spermatogenesis specific driver constructs was conducted by amplifying tTA_SV40 from a pSL_af_Sry $\alpha$ _tTA-SV40_af plasmid ${ }^{15}$ with MID\#138/HM049 primers in a $50 \mu \mathrm{l}$ PCR mixture consisting of water $29 \mu \mathrm{l}$, pSL template $1 \mu \mathrm{l}(50 \mathrm{ng} / \mu \mathrm{l}), 5 \mathrm{x}$ Phusion buffer $10 \mu 1$, Phusion polymerase $1 \mu 1$, dNTPs $4 \mu 1$, forward primer $2.5 \mu 1$ and reverse primer $2.5 \mu \mathrm{l}$ in a PCR program of $2 \mathrm{~min}$ at $98^{\circ} \mathrm{C}, 30 \mathrm{sec}$ at $98^{\circ} \mathrm{C}, 30 \mathrm{sec}$ at $68^{\circ} \mathrm{C}, 1 \mathrm{~min}$ at $72^{\circ} \mathrm{C}$ 35 cycles, $7 \mathrm{~min}$ at $72^{\circ} \mathrm{C}$. The amplified fragment was run on $1 \%$ agarose gel and distinct single band was excised and purified.

About $1 \mu \mathrm{g}$ each of the amplified tTA_SV40 and a pSL_af_attP_B2t_DsRed plasmid were cut using ClaI/HindIII in separate $50 \mu \mathrm{l}$ reactions using standard digestion protocol. The digested fragment and plasmid were run on $1 \%$ agarose gel separately and the distinct single bands obtained were excised, purified, and measured. The purified fragments were cloned by ligating their cut ends after removing DsRed fragment from the pSL plasmid. The final construct generated after ligation resulted into pSL_af_attP_ 32 t_tTA_SV40_af which was subsequently sent for sequencing and thereby verified.

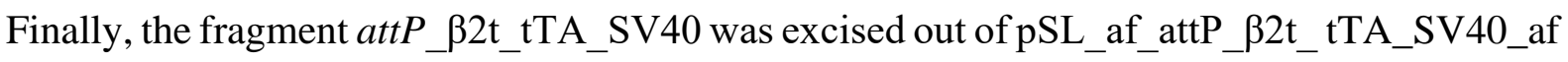
shuttle vector via AscI digestion and inserted into AscI-cut, and purified pBac \{3xP3_EGFP_SV40_af ${ }^{28}$ transformation vector. The cut fragment attP_ 32 t_tTA_SV40 was run on $1 \%$ agarose gel and distinct single band was excised and purified as stated above. The fragment was ligated to the transformation vector to generate pBac \{a_3xP3_EGFP_SV40 _attP_B2t_tTA_SV40\}.

\section{Generation of Cas9 expressing responder construct.}

In order to generate the pBac $\{$ a_3xP3_DsRed_SV40_attP_TRE_hsp68_Cas9_SV40 Cas9\}, Cas9 responder construct: firstly Tchsp68 fragment was amplified from pBac \{a_3xP3_DsRed_SV40_TRE_Tchsp68_Tc-intron_tGFP_SV40\} plasmid with 
Tc_hsp68_F_KpnI and Tc_hsp68_R_ClaI primers in a 50 $\mu 1$ PCR mixture consisting of water 28.5 $\mu 1, p B a c\{$ a_3xP3_DsRed_SV40_TRE_Tchsp68_Tc-intron_tGFP_SV40 $\}$ plasmid $1.5 \mu 1$ (50ng/ $\mu 1), 5 x$ Phusion buffer $10 \mu 1$, Phusion polymerase $1 \mu 1$, dNTPs $4 \mu 1$, forward primer $2.5 \mu 1$ and reverse primer $2.5 \mu \mathrm{l}$ in a PCR program of $2 \mathrm{~min}$ at $98^{\circ} \mathrm{C}, 30 \mathrm{sec}$ at $98^{\circ} \mathrm{C}, 30 \mathrm{sec}$ at $58^{\circ} \mathrm{C}$, 5cycles, $30 \mathrm{sec}$ at $98^{\circ} \mathrm{C}, 1 \mathrm{~min}$ at $72^{\circ} \mathrm{C} 30$ cycles, $7 \mathrm{~min}$ at $72^{\circ} \mathrm{C}$. The amplified fragment Tchsp68 was run on $1 \%$ agarose gel and distinct a single band was excised and purified.

About $1 \mu \mathrm{g}$ each of the amplified Tchsp68 fragment and a pSL_af_ attP_TRE_hsp43_Cas9_SV40 plasmid were cut using KpnI/ClaI in separate 50 $\mu 1$ reactions using standard digestion protocol. The reactions were run on $1 \%$ agarose gel and the distinct single bands were excised separately and purified as above. The purified fragments were then cloned by ligating their cut ends after removing hsp43 fragment from the pSL plasmid and replaced with Tchsp68 to generate a final construct of pSL_af_ attP_TRE_Tchsp68_Cas9_SV40_af which was subsequently sent for sequencing by ${ }^{29}$ and thereby verified using geneious 9.1 .8 software.

Finally, the fragment attP_TRE_Tchsp68_Cas9_SV40 was excised out of pSL_af_attP_TRE_hsp68_Cas9_SV40_af shuttle vector via AscI digestion. Also, pBac $\left\{3 \mathrm{xP3}\right.$ _DsRed_SV40 af ${ }^{30}$ fluorescent transformation eye marker was AscI-cut. The cut

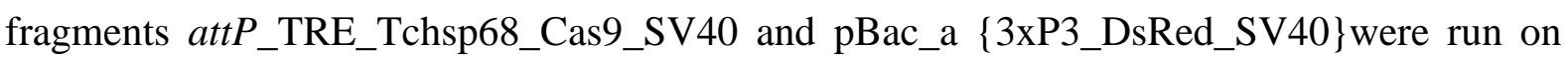
$1 \%$ agarose gel and distinct single bands were excised and purified as described above. The fragments were ligated together to generate $\mathrm{pBac}\left\{\mathrm{a}_{-} 3 \mathrm{xP} 3_{-}\right.$DsRed $\_\mathrm{SV} 40$ attP_TRE_hsp68_Cas9_SV40 Cas9\}vector.

\section{General protocol for cloning:}

PCR amplifications during this study were performed using Phusion DNA polymerase and Phusion-HF buffer (New England Biolabs GmbH, D-65926 Frankfurt am Main). Plasmid isolation and PCR purification using NucleoSpin ${ }^{\circledR}$ Plasmid and NucleoSpin ${ }^{\circledR}$ Gel and PCR Clean-up kits (Macherey-Nagel GmbH \& Co., 52355 Dueren, Germany), respectively. Plasmids for germline transformation were prepared using QIAGEN Plasmid Plus Midi Kit (QIAGEN GmbH, 40724 Hilden, Germany). 


\section{Germline transformation.}

Embryonic injection was done by FemtoJet ${ }^{\circledR}$ Microinjector (Eppendorf, Hamburg, Germany). Needles for the microinjection was made from $10 \mathrm{~mm} \times 1 \mathrm{~mm}$ borosilicate capillaries by pulling them with a P-2000 micropipette puller (Sutter Instrument, Novato USA) with the following settings: Heat $=350$, Fil $=4$, Vel $=50$, Del $=225, \mathrm{PUL}=150$. The pulled needles were opened and sharpened using a Bachofer Laboratoriumsgeräte beveller (Reutlingen, Germany).

To generate driver and responder lines, by piggyBac mediated germline transformation ${ }^{31}$, the constructs $\quad$ pBac_a $\quad\left\{3 x P 3 \_E G F P \_S V 40 \quad\right.$ attP_B2t_tTA_SV40 $\}$ and pBac_a\{3xP3_EGFP_SV40_attP_TRE_hsp68_Cas9_SV40\} were injected into 1000 (0$2 \mathrm{hrs}$ old) embryos, using the following concentrations: piggyBac constructs $500 \mathrm{ng} / \mu \mathrm{lmixed}$ with $300 \mathrm{ng} / \mu \mathrm{l}$ hyperactive helper plasmid mhypbase ${ }^{7}$ into embryos of the Wild type $v^{w}$ strain. To collect the wild type embryos, $v^{w}$ strain were placed on white flour for one hour after which the embryos were collected and kept for one more hour at room temperature and subsequently washed twice in $1 \%$ Klorix solution ${ }^{7}$.

After injection, the embryos were placed onto apple agar plates and sealed in a plastic box, kept humid at $32^{\circ} \mathrm{C} / 90^{\circ} \mathrm{F}$ for 2 days. The injected embryos were transferred to lower humidity afterwards until hatching. Hatched larvae were picked with a hair thin brush individually and placed on full wheat flour until pupation. Each $\mathrm{G}_{0}$ pupal was sexed and individually crossed to three non-injected $v^{w}$ wildtype individuals of the opposite sex.

\section{Dissection of testes for whole mount In-Situ hybridization against tTA}

Dissection of testes was done by placing adults on ice to immobilize them for about 10 minutes. The male individual to be dissected was picked using a forcep and placed in a pool of insect saline solution ${ }^{27}$. By slightly squeezing the thorax of the individual with a pair of forcep the genitalia were exposed, and using another pair of forcep, the exposed testes ${ }^{32}$ were pulled out and moved into iced cold 1xPBS before fixation. Fixation was done following the Dissecting Tribolium Genitalia protocol (http://coleoguy.blogspot.com/2011/10/dissecting-triboliumgenitalia.html) ${ }^{27}$. 


\section{Test crosses to evaluate binary expression system during spermatogenesis}

We made crosses between individual driver lines F5_F1, F5_F2, F10_F3 and F10_M1 (Fig: 3) and the TRE_Cas9 responder line F8_F1 (Fig: 4) and kept until they laid embryos. The embryos were then collected on separate flour and kept until adult emergence. The male offspring that showed double florescence in the eyes (DsRed and EGFP) were selected and their testes ${ }^{32}$ dissected and fixed as shown above. The dissected testes (s) were used for WMISH with a Cas9 anti-sense probe.

\section{Whole Mount in situ Hybridization (WMISH):}

WMISH using DIG-labelled RNA antisense probe against tTA and/or Cas9 on matured testes was performed as reported ${ }^{33}$. The tTA and Cas9 probes were a gift from Ahmed .

\section{Microscopy/Screening:}

First filial generation of adult beetles were screened for possible transgenic beetles with expected EGFP or DsRed expression in the eyes using LEICA M205 FA epifluorescence stereomicroscope (Leica Mikrosysteme Vertrieb Gmb, Wetzlar, 35578 Germany) with the filter sets EGFP-LP (excitation: ET480/40, emission: ET510 LP) and RFP (excitation: ET546/10x, emission: ET605/70m) respectively. Whole testes imaging was done using Image acquisition with cy3 filter, 8bit mono and black/white, and also DIC filter, 24bit color and RGB settings of a Zeiss Axioplan 2 microscope. 


\section{DISCUSSION:}

The Sterile Insect Technique (SIT) is a species specific effective and ecologically safe method of pest control that presents no harm to natural enemies and beneficial insects ${ }^{4}$, and which can be used as a component of an Area-Wide pest management programs to reduce pest population. The inundative release of sterile versions of the organism ${ }^{34}$ for mating with con-specific female, thus leads to infertile mating and significant reduction in pest population ${ }^{1}$. The sterility in classical approach is induced by exposing them to ionizing radiation such as Cobalt-60 or Caesium-137 ${ }^{3}$ 35. Radiation however, affects the fitness of the released sterile organism ${ }^{30}$ because apart from damage the radiation do to the germline cells, which is desirable for use in SIT program, it also affects somatic cells ${ }^{37}$ thus affecting the fitness of the released organisms. Therefore, to overcome the fitness problem of ionized radiated males, the released are done in large numbers ${ }^{38}$ and at intervals to overwhelm the wild male population in the targeted pest control areas.

To solve the attendant drawback with ionization radiation, we proposed a reproductive sterility system ${ }^{9}$ in the coleopteran insect pest $T$. castaneum using the CRISPR/Cas9 ${ }^{39}$ endonuclease system, which is geared towards targeting by plethora of Double Strand Breaks (DSBs) ${ }^{26}$ of certain loci in the sperm chromosomes during spermatogenesis. This will hitherto cause the production of sperm that are not capable of producing viable progeny due to the incorrect repair of the chromosome during the cell repair mechanism, hence causing aneuploidy. However, the system is designed in such a manner that it will not present any fitness cost to the individuals since no somatic cells will be affected.

To achieve this process and as a proof of concept, we designed and generated two different constructs to produce tTA expressing transgenic driver and Cas9 expressing responder lines to be expressed in a conditional tissue specific manner. The conditionality is based on the tet-off expression system ${ }^{10}$. The system is developed using the: 1) P/E of the spermatogenesis specific gene $40 \mathrm{Tc}-32 \mathrm{t}$, which we used to generated the driver construct, which mediates tTA expression in the testes; 2) coding sequence of Cas9 downstream of Tetracycline Responder Element (TRE) to express the Cas9 protein and 3) guides for para-centromeric, sub-telomeric genomic regions/or repetitive sequences ${ }^{24}$.

We performed crosses after generating the strains between the generated driver lines mediating tTA in the testis driven by spermatogenesis specific $\beta 2 \mathrm{t}$ P/E region, to the potential Cas9 effector line. We did not identified any Cas9 specific expression pattern in the testis of the 
progenies of the crosses by WMISH, and this could be attributed to maybe because only a single TRE-Cas9 (F8_F1) responder strains used, hence the need for the generation of more strains and test the expression. Other factors could be related to the possible early shut down of transcription in early maturing primary spermatocytes ${ }^{41}$. However, the use of bam gene P/E which is expressed at meiotic arrest loci in a GAL4/UAS system have being found to be effective in drosophila ${ }^{41}$. Thus, in order to circumvent these possible situations, we intend to generate more Cas9 expressing effector lines, and also to determine the ortholog(s) of bam gene in tribolium and use the $\mathrm{P} / \mathrm{E}$ region to generate lines that will mediate the expression of tTA in early primary spermatocytes.

The proof of concept if successful, will be the first time that CRISPR/Cas9-induced reproductive sterility system, together with sperm marking described in (chapter 1) and female specific embryonic lethality system to be established will be employ in a SIT system for any coleopteran insect pest. This will subsequently serve as a good management practice to some of the members of the largest group of arthropod insect pests such as the Red Palm Weevil (RPW) and as a substitute too for the predominant use of synthetic chemicals. 


\section{FIGURES:}
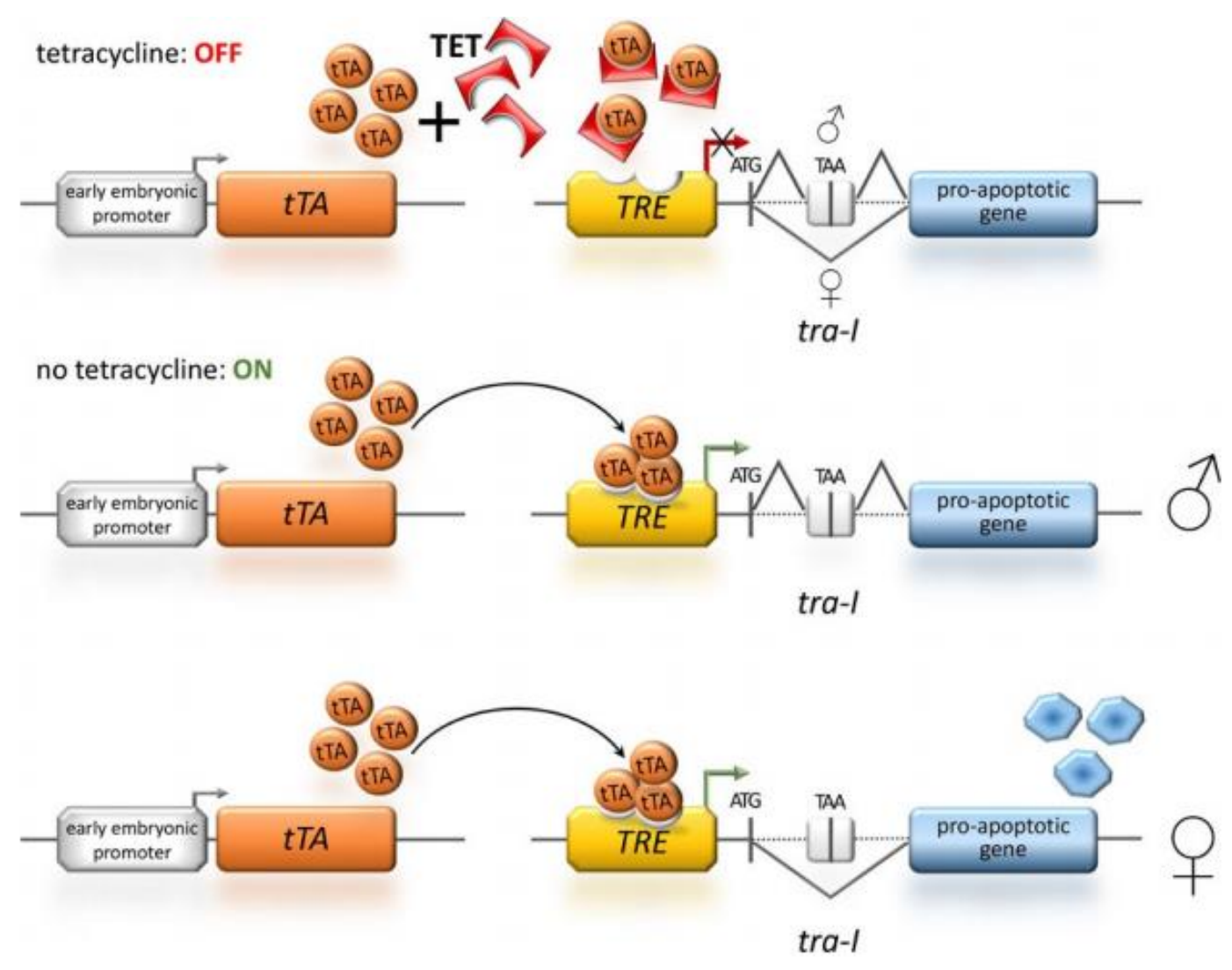

Fig 1: Schematic depiction of transgenic sexing using female-specific splicing under the control of the repressible tTA-system ${ }^{9}$. 

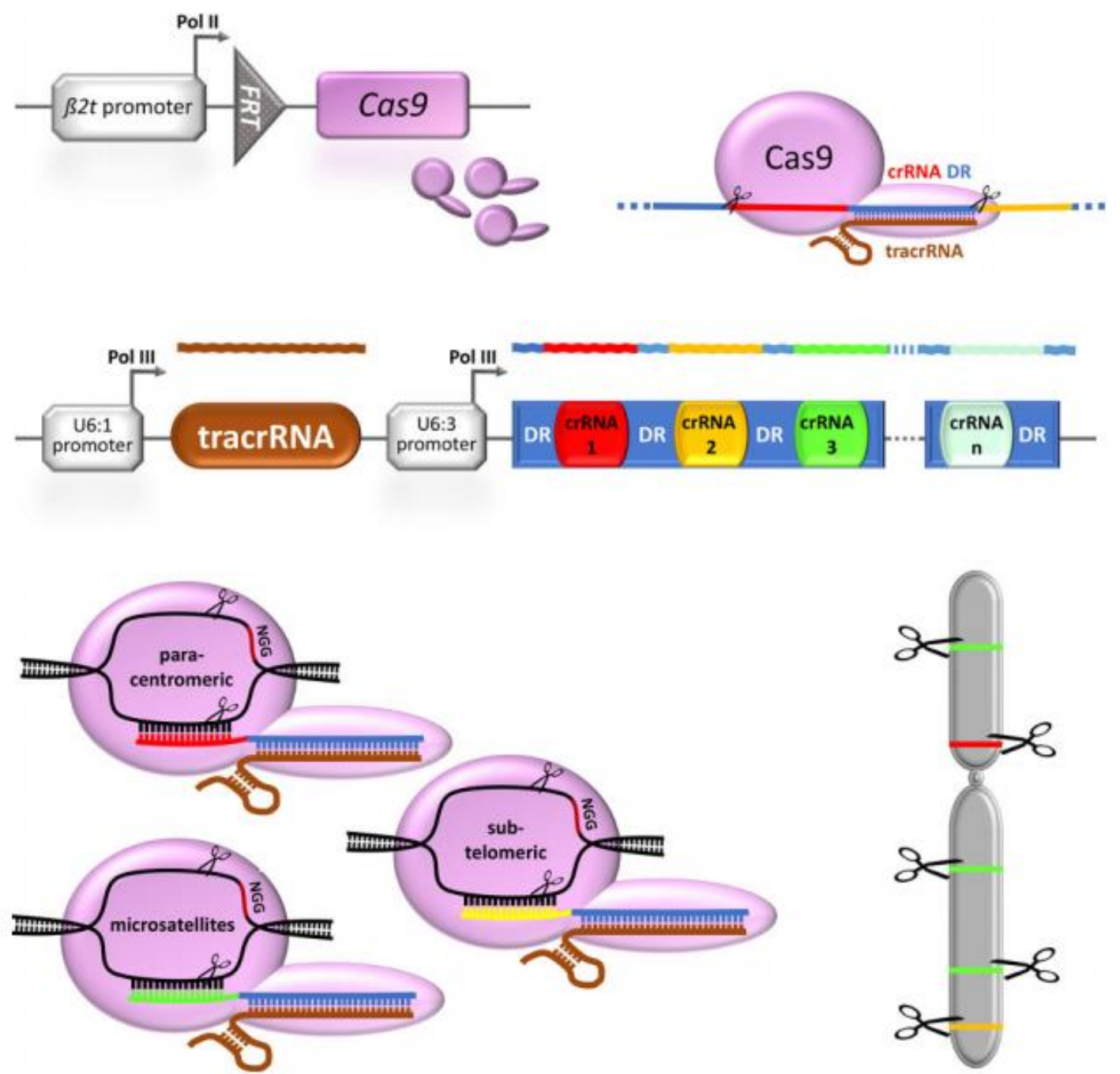

Fig 2: schematic depiction of multifactorial reproductive sterility based on the CRISPR/Cas9 system causing chromosome shredding ${ }^{9}$. 
A)

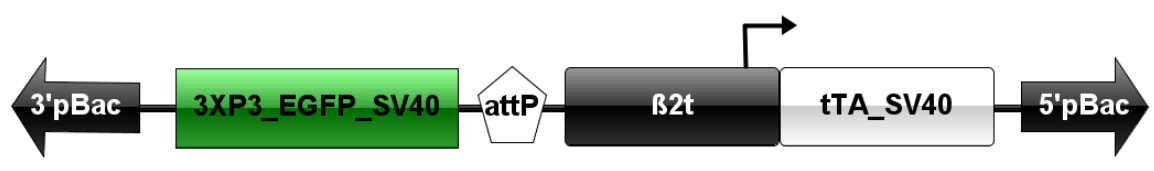

B)

Wildtype 32 -tubulin probe

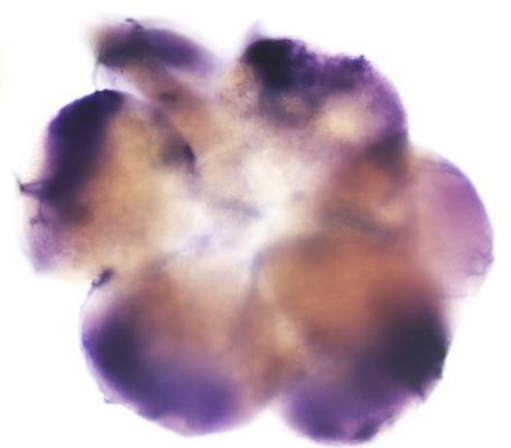

F5_F2

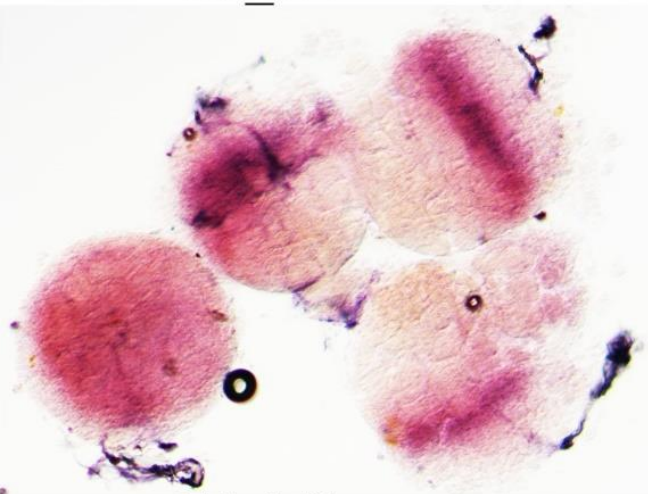

F5_M1

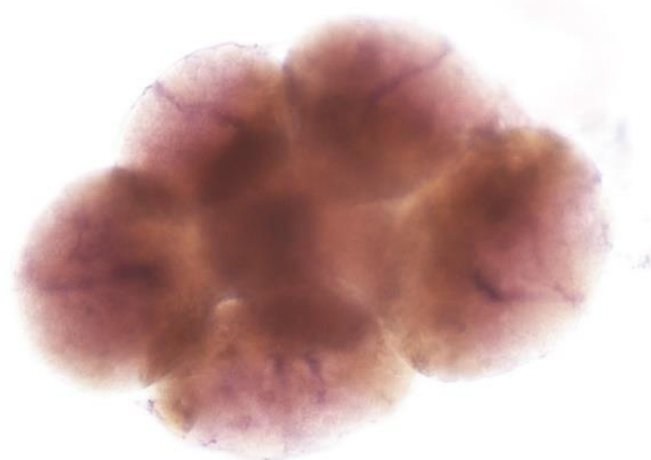

Wildtype tTA probe

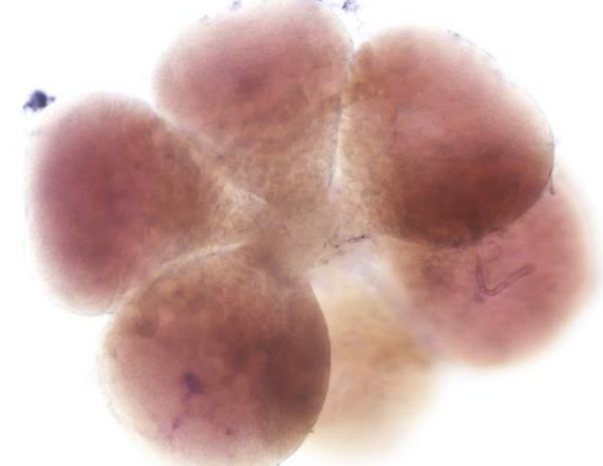

F10_F3

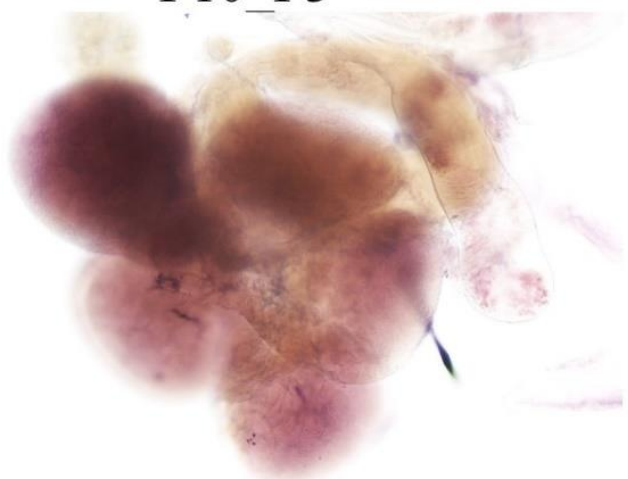

F10 M1

Fig 3: WMISH of Wildtype and transgenic pBac $\left\{3 x P 3 \_E G F P \_S V 40 \_a t t P \_\beta 2 t \_t T A \_S V 40 a\right\}$ driver lines testes

against tTA DIG antisense probe 
A)
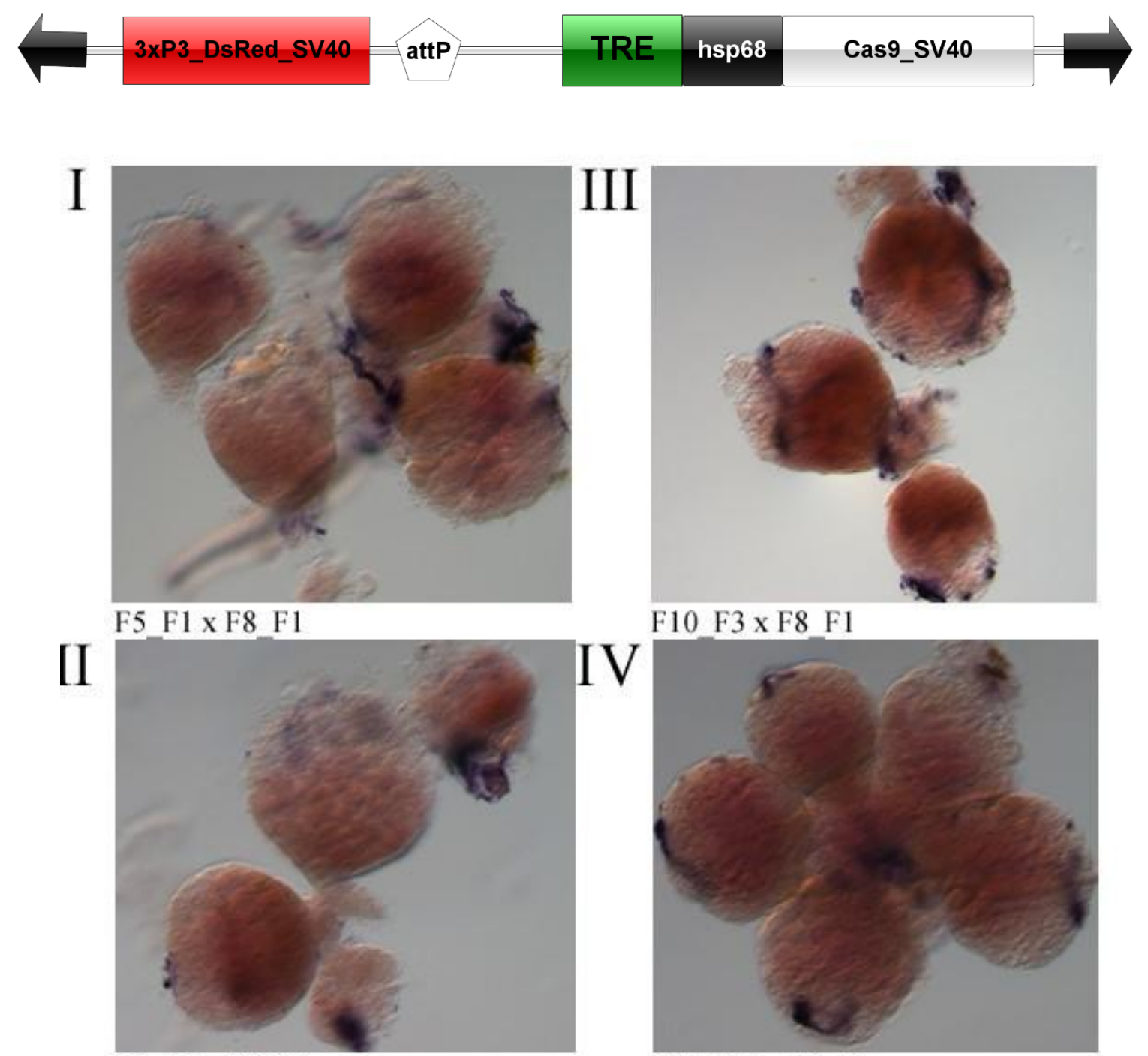

F10 F3 x F8 F1

F5_F2 x F8 F1

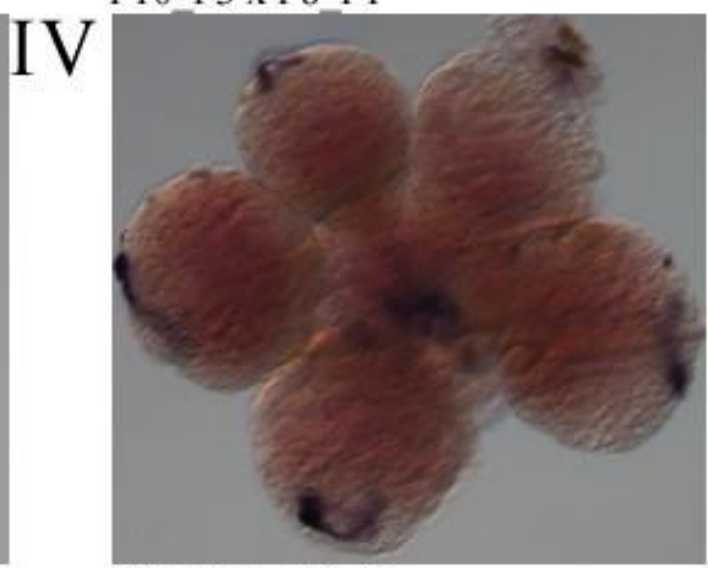

F10_M1 x F8_F1

Fig 4: WMISH of testes in crosses between different driver strains with Cas9 effector strain to detect the expression in the testes by a Cas9 antisense probe. (I) F5_F1 x F8_F1 (II) F5_F2 x F8_F1 (III) F10_F3 x F8_F1 (IV) F10_M1 x F8_F1. The result of the WMISH show no specific gene expression observed 


\section{REFERENCES:}

1. Klassen, W. \& Curtis, C. F. History of the Sterile Insect Technique. in Sterile Insect Technique: Principles and Practice in Area-Wide Integrated Pest Management (eds. Dyck, V. A., Hendrichs, J. \& Robinson, A. S.) 3-36 (Springer Netherlands, 2005). doi:10.1007/1-4020-4051-2_1.

2. Knipling, E. F. Possibilities of Insect Control or Eradication Through the Use of Sexually Sterile Males. J Econ Entomol 48, 459-462 (1955).

3. Ernawan, B., Tambunan, U. S. F., Sugoro, I. \& Sasmita, H. I. Effects of gamma irradiation dose-rate on sterile male Aedesaegypti. in 020010 (2017). doi:10.1063/1.4985401.

4. Dyck, V. A., Hendrichs, J. \& Robinson, A. S. Sterile Insect Technique: Principles and Practice in Area-Wide Integrated Pest Management. (Springer Science \& Business Media, 2006).

5. Hendrichs, J., Franz, G. \& Rendon, P. Increased effectiveness and applicability of the sterile insect technique through male-only releases for control of Mediterranean fruit flies during fruiting seasons. Journal of Applied Entomology 119, 371-377 (1995).

6. Wimmer, E. A. Eco-friendly insect management. Nat Biotechnol 23, 432-433 (2005).

7. Eckermann, K. N. et al. Hyperactive piggyBac transposase improves transformation efficiency in diverse insect species. Insect Biochemistry and Molecular Biology 98, 1624 (2018).

8. Visarada, K., Cr, A., Srujana, S., Nammi, S. \& Seetharama, N. Transgenic Breeding: Perspectives and Prospects. Crop Science 49, (2009).

9. Eckermann, K. N. et al. Perspective on the combined use of an independent transgenic sexing and a multifactorial reproductive sterility system to avoid resistance development 
against transgenic Sterile Insect Technique approaches. BMC Genet 15 Suppl 2, S17 (2014).

10. Das, A. T., Tenenbaum, L. \& Berkhout, B. Tet-On Systems For Doxycycline-inducible Gene Expression. Curr Gene Ther 16, 156-167 (2016).

11. Gossen, M. \& Bujard, H. Tight control of gene expression in mammalian cells by tetracycline-responsive promoters. Proc. Natl. Acad. Sci. U.S.A. 89, 5547-5551 (1992).

12. Urlinger, S. et al. Exploring the sequence space for tetracycline-dependent transcriptional activators: novel mutations yield expanded range and sensitivity. Proc. Natl. Acad. Sci. U.S.A. 97, 7963-7968 (2000).

13. Bello, B., Resendez-Perez, D. \& Gehring, W. J. Spatial and temporal targeting of gene expression in Drosophila by means of a tetracycline-dependent transactivator system. 10.

14. Catteruccia, F., Crisanti, A. \& Wimmer, E. A. Transgenic technologies to induce sterility. Malar J 8, S7 (2009).

15. Horn, C. \& Wimmer, E. A. A transgene-based, embryo-specific lethality system for insect pest management. Nature Biotechnology 21, 64-70 (2003).

16. Schetelig, M. F., Caceres, C., Zacharopoulou, A., Franz, G. \& Wimmer, E. A. Conditional embryonic lethality to improve the sterile insect technique in Ceratitis capitata(Diptera: Tephritidae). BMC Biol 7, 4 (2009).

17. Schetelig, M. \& Handler, A. A transgenic embryonic sexing system for Anastrepha suspensa (Diptera: Tephritidae). Insect biochemistry and molecular biology 42, 790-5 (2012).

18. Shen-Orr, S. S., Pilpel, Y. \& Hunter, C. P. Composition and regulation of maternal and zygotic transcriptomes reflects species-specific reproductive mode. Genome Biology 11, R58 (2010). 
19. Khan, S. A., Eggleston, H., Myles, K. M. \& Adelman, Z. N. Differentially and Coexpressed Genes in Embryo, Germ-Line and Somatic Tissues of Tribolium castaneum. G3 (Bethesda) 9, 2363-2373 (2019).

20. Perspective on the combined use of an independent transgenic sexing and a multifactorial reproductive sterility system to avoid resistance development against transgenic Sterile Insect Technique approaches. - Abstract - Europe PMC. https://europepmc.org/article/med/25471733.

21. Catteruccia, F., Benton, J. P. \& Crisanti, A. An Anopheles transgenic sexing strain for vector control. Nature Biotechnology 23, 1414-1417 (2005).

22. Rendón, P., McInnis, D., Lance, D. \& Stewart, J. Medfly (Diptera: Tephritidae) genetic sexing: large-scale field comparison of males-only and bisexual sterile fly releases in Guatemala. J. Econ. Entomol. 97, 1547-1553 (2004).

23. Xu, Y. \& Li, Z. CRISPR-Cas systems: Overview, innovations and applications in human disease research and gene therapy. Computational and Structural Biotechnology Journal 18, 2401-2415 (2020).

24. Barrangou, R. \& Marraffini, L. A. CRISPR-Cas systems: prokaryotes upgrade to adaptive immunity. Mol Cell 54, 234-244 (2014).

25. Pavlek, M., Gelfand, Y., Plohl, M. \& Meštrović, N. Genome-wide analysis of tandem repeats in Tribolium castaneum genome reveals abundant and highly dynamic tandem repeat families with satellite DNA features in euchromatic chromosomal arms. DNA Res 22, 387-401 (2015).

26. Richards, S. et al. The genome of the model beetle and pest Tribolium castaneum. Nature 452, 949-955 (2008).

27. Brunner, E. et al. CRISPR-induced double-strand breaks trigger recombination between homologous chromosome arms. Life Science Alliance 2, (2019). 
28. Dissecting Tribolium Genitalia. http://coleoguy.blogspot.com/2011/10/dissectingtribolium-genitalia.html.

29. Horn, C. \& Wimmer, E. A versatile vector set for animal transgenesis. Development genes and evolution 210, 630-7 (2001).

30. Microsynth Webshop. https://srvweb.microsynth.ch/.

31. Horn, C., Schmid, B. G. M., Pogoda, F. S. \& Wimmer, E. A. Fluorescent transformation markers for insect transgenesis. Insect Biochem. Mol. Biol. 32, 1221-1235 (2002).

32. Berghammer, A. J., Klingler, M. \& A. Wimmer, E. A universal marker for transgenic insects. Nature 402, 370-371 (1999).

33. Sokoloff, A. The biology of Tribolium: with special emphasis on genetic aspects. (Clarendon Press, 1972).

34. Trauner, J. et al. Large-scale insertional mutagenesis of a coleopteran stored grain pest, the red flour beetle Tribolium castaneum, identifies embryonic lethal mutations and enhancer traps. BMC Biol 7, 73 (2009).

35. Chidawanyika, F., Mudavanhu, P. \& Nyamukondiwa, C. Biologically Based Methods for Pest Management in Agriculture under Changing Climates: Challenges and Future Directions. Insects 3, 1171-1189 (2012).

36. Zhao, J. et al. Sperm precedence pattern and the effect of irradiation on male mating competition in the oriental fruit fly, Bactrocera dorsalis. 5.

37. Guerfali, M. M. et al. Fitness and Reproductive Potential of Irradiated Mass-Reared Mediterranean Fruit Fly Males Ceratitis capitata (Diptera: Tephritidae): Lowering Radiation Doses. flen 94, 1042-1050 (2011).

38. Helinski, M. E., Parker, A. G. \& Knols, B. G. Radiation biology of mosquitoes. Malar J 8, S6 (2009). 
39. Urquidi, J., Brar, R. K., Rodriguez, S. \& Hansen, I. The development of new radiation protocols for insect sterilization using long wavelength x-rays. in 020010 (2015). doi:10.1063/1.4927187.

40. Siebert, K. S., Lorenzen, M. D., Brown, S. J., Park, Y. \& Beeman, R. W. Tubulin superfamily genes in Tribolium castaneum and the use of a Tubulin promoter to drive transgene expression. Insect Biochemistry and Molecular Biology 38, 749-755 (2008).

41. White-Cooper, H. Tissue, cell type and stage-specific ectopic gene expression and RNAi induction in the Drosophila testis. Spermatogenesis 2, 11-22 (2012). 
Table 1: Primer sequences

\begin{tabular}{|l|l|l|}
\hline CODE & NAME & SEQUENCES 5' $-\mathbf{3}$ \\
\hline MID\#203 & Tc-B2t_probe_F & CCGAAAGAGGCGGCAGTTTTGAAACAAC \\
\hline MID\#204 & Tc-B2t_probe_R & CTACGAGCTGATGTACGGAGAGGGTGGC \\
\hline & pJet 1.2 forward & CGACTCACTATAGGGAGAGCGGC \\
\hline MID\#138 & ClaI-tTA-F-CDS & $\begin{array}{l}\text { GAGGATCGATATGTCTAGATTAGATAAAAGTAAAGTGATTAA } \\
\text { CAG }\end{array}$ \\
\hline & HM049 & CGAAAGGGGGATGTGCTGCAAGGCGATTAAG \\
\hline MID\#115 & Tc_hsp68_F_Kpn1 & GAGGTACCCGTTTCATATATAAGCGCGGTCTCG \\
\hline MID\#116 & Tc_hsp68_R_Cla1 & GCCGATCGATACTTTGAATTCACTAGTAAATAATTCAC \\
\hline & & \\
\hline & & \\
\hline
\end{tabular}

Table 2: LIST OF VECTORS

\section{COMPONENT OF CONSTRUCT}

pSL_af_Srya_tTA-SV40_af

pSL_af_attP_ $\beta 2$ t

$\mathrm{pBac}\left\{\mathrm{a} \_3 \times \mathrm{xP} 3 \_\right.$EGFP_SV40_attP $\beta 2 \mathrm{t}$ tTA_SV40 $\}$

pSL_af_attP_TRE_hsp43_Cas9_SV40

pSL_af_attP_TRE_Tchsp68_Cas9_SV40_af

pBac $\left\{\mathrm{a} \_3 \times \mathrm{XP3}\right.$ _DsRed_SV40_attP_TRE_hsp68_Cas9_SV40 Cas9\} 
3.4 Genetic improvement and use of CRISPR/Cas9 to generate an early embryonic driver line in Tribolium castaneum

In this chapter $\mathrm{i}$ used CRISPR/Cas9 system to generate a driver strain using the newly discovered Tc007675 early Tribolium early embryonic gene P/E region to drive tTA in early embryos using a bi-cistronic construct. Also, I intend to create mutants to investigate the functionality of the gene in situ.

ISAH, Musa Dan'azumi \& Ernst A. Wimmer

Authors contribution to the practical work

ISAH, Musa Dan'azumi: Conceived and design the experiment, conducted all the other experiment and analysis. Wrote the manuscript.

Status: Work in progress 
Genetic improvement and use of CRISPR/Cas9 to generate an of early embryonic driver line in Tribolium castaneum

ISAH, Musa Dan'azumi \& Ernst A. Wimmer

1) Department of Developmental Biology, Johann-Friedrich-Blumenbach-Institute of Zoology and Anthropology, Göttingen Center for Molecular Biosciences, Georg-August-University Göttingen, 37077 Göttingen, Germany.

2) Department of Crop Science, Faculty of Agriculture, Wildlife and Forestry Resources Management, University of Calabar, P.M. B. 1152, Calabar, Cross River State, Nigeria.

* Corresponding Author: E. A. Wimmer. Tel. +49 5513928666

E-Mail address: ewimmer@gwdg.de.

\begin{abstract}
:
CRISPR-Cas is an RNA guided endonuclease defense system use by bacterial and other archaeal organism to cleave invading foreign DNA materials in their system. The system is comprising of human codon optimized Cas9 and their required RNA components. Cas 9 can be directed to any target gene sequence by modifying the single-guide (sgRNA) sequence to align with the Protospacer Adjacent Motif (PAM) sequence of the gene, which subsequently results to Double Strand Breaks (DSBs). DSBs at a specific locus in the genome stimulates subsequent genome editing via Non-Homologous End Joining (NHEJ) or Homology Directed Repair (HDR). Using the HDR system, we generated a transgenic driver strain at the Tribolium castaneum by modifying the Tc007675 gene locus, which was identified to be expressed during early embryonic cellularization to mediate the expression of tTA by Whole Mount in Situ Hybridization (WMISH). Result showed the detection of tTA expression in cytoplasm of the transgenic embryos, though not as strong as the indigenous expression of the Tc007675 by Whole Mount in Situ Hybridization (WMISH). This driver strain expresses the heterologous transactivator (tTA) which thus can be used to establish reproductive sterility or transgenic sexing/lethality systems in beetle. In addition, we intend to create mutants for Tc007675 gene by using Recombinase Mediated Cassette Exchange (RMCE) approach.
\end{abstract}

Keywords: CRISPR-Cas9, Tribolium castaneum, Tc007675, RMCE 


\section{INTRODUCTION:}

CRISPR-Cas is a novel genome editing system approach of cleaving the DNA of invading foreign body by using nucleases that are RNA guided ${ }^{1}$. It is diverse and found to be used in several bacteria and archaeal organism ${ }^{1}$. It is a complex system that comprises of CRISPRassociated (Cas) genes ${ }^{2}$ and many repetitive elements ${ }^{1}$, and within the array, are a group of repetitive sequences term as protospacers ${ }^{2}$, that are derived from the target DNA which are always associated with the Proto Adjacent Motif (PAM) sequence ${ }^{1-3}$.

CRISPR - Cas is a RNA- guided nuclease activity comprising of the expression of optimized Cas9 nuclease and its component ${ }^{3}$, the nuclease activity is then achieved via cell repair mechanism such as Non Homologous End Joining (NHEJ) or Homology Directed Repair (HDR) ${ }^{6}$. It also involves the redirecting Cas9 is to any target of interest close to the PAM sequence ${ }^{6}$ which subsequently results in DNA Double Strands Breaks (DSB) at specific loci of interest ${ }^{6}$.

Genomic DNA of any organism is prone to double strand breaks at any point due to several natural mechanism ${ }^{7}$. These breaks that arises are normally repaired via the cell machinery network to maintain the genomic integrity. The mechanism used are the NHEJ or HDR ${ }^{8-10}$, while the NHEJ evolved around a mechanistic flexibility ${ }^{4}$ which can lead to insertion or deletions (indels) resulting to premature stop codons or frameshifts ${ }^{5}$ in the genomic sequences resulting into a loss-of-function mutations. The HDR on the other hand also a natural occurring nucleic acid repair system ${ }^{2}$, requires a repair template that have the modified sequence of interest flanked by homology arms to direct homology directed repair of the DSB ${ }^{6}{ }^{16}$.

Site-specific transgene integration system is a genome modification strategy were specific artificially engineered nucleotide sequence is integrated and targeted within the genome of an organism via recombination ${ }^{8}$. Examples of site-specific recombinant systems are, Cre-loxP from bacteriophage P1, Flp-FRT from Saccharomyces cerevisiae and phiC31-att from a Streptomyces bacteriophage ${ }^{7-9}$.

Introduction of docking site ${ }^{11-12}$ such as 'attP' using varying molecular biology approach such as transposable ${ }^{3-4}$ element-based transgene or CRISPR-Cas9 ${ }^{1-2}$ is the first step in Sitespecific recombinase experiment . Using molecular biology approach the transgenes of interest carrying an attB site is then integrated into the docking site using any recombination enzyme ${ }^{8}$. 
phiC31 integrase is used between attB (carrying transgenes of interest) and attP (introduced as the docking site) sites, creating attL and attR hybrid junctions ${ }^{7}$.

The hybrid sites are no longer accessible to the enzyme after the recombination activity, thereby making the transgenesis stable and efficient ${ }^{7}$, also since the hybrid sites are not found in nature ${ }^{11}$ it makes the system efficient. The system can be achieved by using either a single or double landing sites. The use of a single landing site however has some observable drawbacks, because when the transgene is delivered predominantly using a plasmid vector, it results in the insertion of the plasmid with it resistance gene ${ }^{12}$. This drawback is however avoided when two landing sites are used by Recombinase Mediated Cassette Exchange (RMCE) ${ }^{12}{ }^{13}$. RMCE is a recombination strategy that uses two recombinase sites which are separated by a marker gene at the landing/docking site ${ }^{12} 13$. RMCE have also being employed for stabilization of previously generated transgene, modification of inserted transgenes and for some site-specific transformation for the study of different transgenes ${ }^{14}$.

Generation of sexing and embryonic lethality binary expression systems ${ }^{14}$ have been proposed for the management of Ceratitis capitata ${ }^{15,16}$, Drosophila melanogaster ${ }^{17}$, Aedes aegypti ${ }^{18}$ etc. The system is expressed in a tet-off conditional manner whereby an apoptotic gene e.g. head involution defective (hid), grim or reaper proteins are overexpressed which led to apoptotic cell death ${ }^{16,19,20}$. However, to facilitate the expression of any of the apoptotic gene in a heterologous transactivator using the binary system which could result in sterility, Female Specific Embryonic Lethality (FSEL) ${ }^{16,21,22}$ or early embryonic lethality ${ }^{20}$, there is the need for the identification, isolation and integration of the recombinant early embryonic cellularization specific $(\mathrm{P} / \mathrm{E})$ genes that can effectively drive the tetracycline transactivator (tTA) through either piggyBac transformation or CRISPR-Cas9 system ${ }^{3}$.

Here we show that the novel gene Tc007675 that is active at the cellularization stage can be engineered using the CRISPR system to a develop driver strain using a bi-cistronic construct that can be used in a heterologous transactivator binary system which could result in sterility, Female Specific Embryonic Lethality or early embryonic lethality systems. Furthermore, using RMCE technique we intend to also create mutant strains for the Tc007675 early embryonic cellularization specifically expressed gene. 


\section{Results:}

\section{Design of guide RNAs, cloning and testing using T7 endonuclease:}

To determine gRNAs that we will use to modify theTc007675 gene locus using CRISPR/Cas9 technique, i used the Tc007675 genomic sequence in the gRNA gene target finder tool. I identified 4 potential gRNA's (G1, G2, G3 and G4) upstream of the 5'UTR and 5 potential gRNA's (G4, G5, G6, G7, G8 and G9) downstream of the 3'UTR (Table 2). We then test the efficiency of each cloned guide RNAs using T7Endol assay. After the assay, gRNA_3 of upstream 5'UTR and gRNA-5 of downstream 3'UTR were found to effectively digestion in the T7Endol assay (Figure 1) and hence kept for further experiment.

\section{Generation of an early embryonic driver line using Crispr/cas9:}

To generate the PCR-LHA-attP-Tc007675(mRNA)-P2A-tTA-3'UTR-3xP3_Tc'V_SV40attP-RHA construct (Fig 2) that will be used to generate driver strains using Homology Directed Repair (HDR). I first obtained a PCRII_tTA plasmid (generated by Ahmed Hassan Mohammed Mutasim), which I used in building a bi-cistronic construct that composed of $1 \mathrm{~Kb}$ each of the upstream and downstream region of the Tc007675 gene which is flanked by two phage attachment sites (attP), the Tc007675 complete gene sequence, tetracycline transactivator (tTA), 3'UTR of Tc007675 gene to ensure the correct folding and termination of the protein, and $\mathrm{Tc}$ vermillion $(\mathrm{Tcv}){ }^{23}$ rescue black eye marker for the identification.

To ensure that the generated plasmid (PCR-LHA-attP-Tc007675 (mRNA)-P2A-tTA-3'UTR3xP3_Tc'V_SV40-attP-RHA (Fig 2) when injected will not be susceptible to cut by the gRNAs in situ when the genome of the organism is modified, i performed a mutagenesis PCR ${ }^{24}$ to modify the gRNAs target sites in a way they cannot be targeted any longer in situ.

To generate the transgenic strain using PCR-LHA-attP-Tc007675 (mRNA)-P2A-tTA-3'UTR3xP3_Tc'V_SV40-attP-RHA (Fig 2) plasmid. We successfully generated one transgenic animal which was screen under a bright field microscope and selected due to its rescue black $T c$ vermillion (Tcv) eye colour. Furthermore, I molecularly characterized the line generated by inverse PCR and sequencing by microsynth ${ }^{25}$, and could confirm a faithful integration of the repair template at the intended genomic site (Fig 2 and Table 3). 


\section{Detection of tTA expression in embryonic driver line generated using CRISPR Cas9:}

To detect cellularization-specific mediated expression of tetracycline trans-activator (tTA) for the embryonic driver line. I performed WMISH against tTA, were transient tTA expression was detected in the cytoplasm of the embryos of the transgenic driver line (Fig 4). Though, it did not seem to be as strong as the endogenous Tc007675 expression in wildtype embryos. The detection of the tTA expression observed indicated that the strain can be used as a driver line for sexing and embryonic lethality systems.

\section{Generation of Tc007675 Mutants using RMCE.}

To generate mutants for the Tc007675 gene using RMCE technique which will give us the opportunity to investigate the function of Tc007675 gene, we injected PCRII-attB3xP3_EGFP_SV40-attB (Fig 5) plasmid with the helper plasmid hsp70-phiC31-nls integrase. However, we are were not able to generate mutants for the gene which could possibly be due to the Drosophila hsp70 used instead of endogenous hsp68 to Tribolium.

\section{Methods}

\section{Nucleic acid isolation:}

All genomic DNA used for this experiment was isolated from a mix sexes of Tribolium castaneum vermilion $^{\text {white }}\left(v^{w}\right)$ using NucleoSpin ${ }^{\circledR}$ DNA Insect (Macherey-Nagel.

\section{Design of guide RNAs, cloning and testing using T7 endonuclease:}

The online fly CRISPR target finder tool (http://tools.flycrispr.molbio.wisc.edu/targetFinder/; ${ }^{26}$ ) was used to identified short guide-RNAs (gRNAs) in the Tc007675 genomic region. mRNA gene sequence for gRNAs was obtained from regions upstream and downstream of the gene coding region while avoiding target sites within the transcribed region of the gene from Tcas 5.2 Tribolium gbrowser (http://bioinf.uni-greifswald.de/gb2/gbrowse/tcas5/) ${ }^{27}$ database.

Four gRNAs (G1, G2, G3 and G4) upstream of the 5'UTR and Five gRNAs (G4 G5, G6, G7, G8 G9) downstream of the 3'UTR with no off-target effect were identified (Table 2). Pairs of complimentary oligonucleotides were synthesized for each identified gRNA scaffold with appropriate overhangs added for cloning into the $B s a \mathrm{I}$ cloning site of $p$ (U6b-BsaI-gRNA) ${ }^{28}$ 
plasmid. Non phosphorylated oligonucleotides were ordered as normal primers and were annealed as reported ${ }^{29}$ and then cloned into $p(\mathrm{U} 6 \mathrm{~b}-B s a \mathrm{I}-\mathrm{gRNA})$ digested with $B s a \mathrm{I}$ using golden gate cloning. The cloning was done in a $25 \mu 1$ reaction comprising of $15 \mu 1$ water, $10 \mathrm{x}$ T4 ligase buffer $2.5 \mu 1$, annealed oligos $3 \mu 1,10 \mathrm{mM}$ ATP $2 \mu \mathrm{l}$ and T4 ligase $1 \mu 1$ which were left at room temperature for 2 hours and subsequently transformed into DH5 $\alpha$.

The efficiency of the cloned guide RNAs were tested using T7Endol assay ${ }^{30}{ }^{31}$. Individual guide RNA plasmids $(500 \mathrm{ng} / \mu \mathrm{l})$ mixed with pSL_af_attP_TRE_Tchsp68_Cas9_SV40_af plasmid (generated as shown in chapter 3) $(500 \mathrm{ng} / \mu \mathrm{l})$ were injected into $75 v^{w}, 2$ hours old preblastoderm embryos. About 20 injected embryos for each guide were taken after 2 days from each batch and placed in a $1.5 \mu 1$ Eppendorf tubes and subsequently crushed using pipette tips. DNA from the crushed embryos were extracted using smashing buffer as described 2932 .

A $50 \mu 1$ PCR reaction using $5 \mu 1$ of the extracted DNA as template and using the different primers (Table) overlapping the gene regions cut by individual gRNAs was conducted. Amplified PCR fragments were excised from agarose gel and subsequently purified, quantified and 500ng/ $\mu \mathrm{l}$ of the product was used for a T7Endol assay. The $500 \mathrm{ng} / \mu \mathrm{l}$ were mixed in $1 \mathrm{x}$ NEB 2.1 buffer in a total $19 \mu 1$ reaction and the subsequent denaturization and rehybridization for a T7Endol assay was conducted ${ }^{33}$. Also, as control wildtype un-injected embryos were used for the T7Endol assay.

\section{Cloning of repair template:}

To generate the bicistronic PCR-LHA-attP-Tc007675 (mRNA)-P2A-tTA-3'UTR3xP3_Tc'V_SV40-attP-RHA (Fig 2c) plasmid, a pair of complimentary P2A oligonucleotides MID\#178/179 were synthesized, annealed in a 100 $\mu 1$ reaction ( $90 \mu 1$ water, $5 \mu 1$ of the forward and reverse $10 \mathrm{mM}$ primers). The reaction was allowed to stand in a heat block set at $95^{\circ} \mathrm{C}$ for 5 min, after which the heat block was switched off and allowed to cool down to room

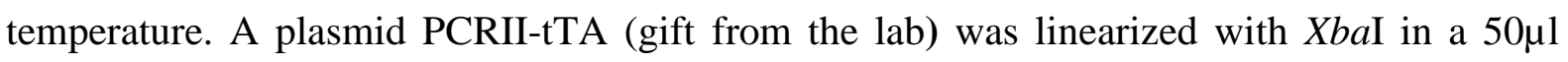
reaction comprising of water $43 \mu 1,10 \mathrm{x}$ cutsmart buffer $5 \mu \mathrm{l}$, and $1 \mu \mathrm{g}$ PCRII-tTA and $1 \mu \mathrm{l}$ $X b a \mathrm{I}$ restriction enzyme. The reaction was kept at $37^{\circ} \mathrm{C}$ for 1 hour after which it was run on $1 \%$ agarose gel and the distinct band excised, purified using PCR clean up and gel extraction kit (Machery Nagel GmbH). Fragment concentration was determine using NanoDrop 2000 Spectrophotometer (Thermo Scientific ${ }^{\mathrm{TM}}$ USA). The linearized plasmid PCRII-tTA was ligated to the annealed $\mathrm{P} 2 \mathrm{~A}$ oligonucleotide in a $25 \mu 1$ reaction made up of $14 \mu 1$ water, $10 \mathrm{x}$ ligation buffer $2.5 \mu 1,10 \mathrm{mM}$ ATP $2.5 \mu 1$, annealed oligos $3 \mu 1$, linearized PCRII-tTA plasmid 
$2 \mu \mathrm{l}$ and $1 \mu \mathrm{l}$ ligase to generate PCRII-P2A-tTA which was kept at room temperature for 1 hour and transformed subsequently into DH $5 \alpha$ competent cells.

The second step of cloning involved the Gibson assembly cloning of 4 different fragments using HIFI DNA cloning kit ${ }^{34}$. The primers for the golden gate cloning were designed using https://goldengate.neb.com/editor ${ }^{35}$ online tool to design the primers (Table 1).

The plasmid PCRII-P2A-tTA was digested with $B s a$ I restriction enzyme in a 50 $\mu 1$ reaction consisting of $40.5 \mu \mathrm{l}$ water, $10 \mathrm{x}$ cutsmart buffer $5 \mu 1,1 \mu \mathrm{g}$ PCR-P2A-tTA plasmid and $1 \mu 1 \mathrm{Bs} a \mathrm{I}$ restriction enzyme. The reaction was kept at $37^{\circ} \mathrm{C}$ for 1 hour, after which the enzyme was inactivated at $65^{\circ} \mathrm{C}$ for $20 \mathrm{~min}$ in a heat block, the product was run on $1 \%$ gel and the band excised, purified by using standard practice and measured using Nanodrop 2000 Spectrophotometer (Thermo Scientific ${ }^{\mathrm{TM}}$ USA).

Afterwards, the 3 other fragments (1Kb Left Homology Arm, Tc007675 mRNA, and attP) were amplified using different pairs of primers with; Left Homology Arm (LHA) fragment using MID\#192/193 primers amplified from pSL_Tc007675 (P/E)_2.1Kb_tTA_SV40 plasmid (generated in section 3.2), Tc007675 mRNA fragment using MID\#188/189 primers amplified from Pjet_Tc007675_mRNA plasmid and attP fragment using MID\#190/191 primers amplified from HM_pBXLII_Ds_Sry2 (generated by Ahmed Hassan Mohammed Mutasim) plasmid.

The amplifications were done in a $50 \mu 1$ reaction consisting of $28 \mu 1$ water, $5 x$ Phusion buffer $10 \mu 1$, dNTPs $4 \mu 1,100 \mathrm{ng} / \mu 1$ of plasmid $2 \mu 1,2.5 \mu 1$ forward and reverse primers each and $1 \mu 1$ Phusion polymerase, with PCR condition of $98^{\circ} \mathrm{C} 2$ min followed by 35 cycles of $98^{\circ} \mathrm{C} 30 \mathrm{sec}$, annealing temperature; Left Homology $\operatorname{Arm}\left(56^{\circ} \mathrm{C}\right), \operatorname{Tc} 007675 \mathrm{mRNA}\left(58^{\circ} \mathrm{C}\right)$, and $\operatorname{att} \mathrm{P}\left(62^{\circ} \mathrm{C}\right)$ $30 \mathrm{sec}$, and $72^{\circ} \mathrm{C} 1 \mathrm{~min}$ with a final elongation $72^{\circ} \mathrm{C}$ for $10 \mathrm{~min}$. The fragments were amplified, run on an $1 \%$ agarose gel and the bands excised, purified by using standard practice and measured using Nanodrop 2000 Spectrophotometer (Thermo Scientific ${ }^{\mathrm{TM}}$ USA).

The amplified and digested fragments were cloned using the golden gate cloning to generate PCR-LHA-attP-Tc007675(mRNA)-P2A-tTA (Fig 1) where the reaction was kept at $50^{\circ} \mathrm{C}$ for 60 min after which $2 \mu 1$ of the mix was transformed in DH5 $\alpha$ NEB competent cells. Three colonies were picked afterwards and inoculated in a mini-prep. The plasmid was subsequently isolated, purified and measured, and test digests using ApaI, BamHI, EcoRI and NcoI restriction enzymes to pick the right clones based on the fragments obtained as verified by geneious 9.1.8 software. The right clone was sent for sequencing ${ }^{25}$ and was further verified. 
The third step involved the Gibson assembly cloning of 5 different fragments comprising of 1Kb Right Homology Arm (RHA), Tc007675 3'UTR, attP and the 3xP3_Tc vermilion ${ }^{\text {white }}$ fragments. Using HIFI DNA cloning kit and the https://goldengate.neb.com/editor ${ }^{35}$ online tool, the primers (Table 1) for the amplification of the fragments were designed.

The generated plasmid PCR-LHA-attP-Tc007675(mRNA)-P2A-tTA (from second cloning step) was digested with BamHI restriction enzyme, while the other fragments were amplified with; Right Homology Arm (RHA) fragment amplified using MID\#180/181 primers amplified from wildtype vermilion ${ }^{\text {white }}$ gDNA, Tc007675 3'UTR fragment amplified using MID\#186/187 primers amplified from Pjet_Tc007675_mRNA plasmid, attP fragment amplified using MID\#182/183 primers amplified from HM_pBXLII_Ds_Sry2 (generated by Ahmed Hassan Mohammed Mutasim) plasmid and 3xP3_Tc vermilion ${ }^{\text {white }} 23$ fragment amplified using MID\#184/185.

The amplifications were done in a $50 \mu 1$ reaction consisting of $28 \mu 1$ water, $5 \mathrm{x}$ Phusion buffer $10 \mu 1$, dNTPs $4 \mu \mathrm{l}, 100 \mathrm{ng} / \mu \mathrm{l}$ of plasmid $2 \mu 1,2.5 \mu \mathrm{l}$ forward and reverse primers each and $1 \mu \mathrm{l}$ Phusion polymerase, with PCR conditions of $98^{\circ} \mathrm{C} 2$ min followed by 35 cycles of $98^{\circ} \mathrm{C} 30$ sec, annealing temperature; Right Homology Arm $\left(57^{\circ} \mathrm{C}\right), 3^{\prime} \mathrm{UTR}\left(58^{\circ} \mathrm{C}\right), 3 \times \mathrm{P} 3$ - Tc vermilion $^{\text {white }}\left(55^{\circ} \mathrm{C}\right)$ and att $\mathrm{P}\left(58^{\circ} \mathrm{C}\right) 30 \mathrm{sec}$, and $72^{\circ} \mathrm{C} 1 \mathrm{~min}$ with a final elongation $72^{\circ} \mathrm{C}$ for $10 \mathrm{~min}$. The fragments amplified, were run on $1 \%$ agarose gel and the distinct bands observed were excised, purified by using standard practice and measured using Nanodrop 2000 Spectrophotometer (Thermo Scientific ${ }^{\mathrm{TM}}$ USA).

The amplified and digested fragments were cloned using the golden gate cloning to generate PCR-LHA-attP-Tc007675(mRNA)-P2A-tTA-3'UTR-3xP3_Tc'V_SV40-attP-RHA (Fig:1) where the reaction was kept at $50^{\circ} \mathrm{C}$ for 60 min after which $2 \mu \mathrm{l}$ of the mix was transformed in a DH5 $\alpha$ NEB competent cells. Eleven colonies were picked and inoculated in a mini-prep and the plasmid was subsequently isolated, purified and measured. Test digests using AvrII, HpaI, EcoRI and NcoI restriction enzymes were used to identify the right clones based on the fragments obtained as verified by geneious 9.1.8 software. The right clone was subsequently sent for sequencing ${ }^{25}$ and could thereby be verified. 


\section{MUTAGENESIS PCR OF REPAIR TEMPLATE:}

For the Right Homology Arm (RHA) mutagenesis, PCR was conducted using MID\#194/195 primers to mutagenize the gRNA-5 recognition site downstream of 3'UTR. While for the Left Homology Arm (LHA), mutagenesis PCR was performed using MID\#196/197 primers to mutagenize the recognition site of gRNA_3 upstream of 5'UTR. We mutagenized ${ }^{24}$ the GGG PAM sequence to TAG for the Left Homology Arm (LHA) the cut site of the gRNA_3 upstream of 5'UTR, and AGG PAM sequence to AGA for the Right Homology Arm (RHA) the cut site of gRNA-5 downstream of 3'UTR while still maintaining the codon usage. The template generated from the mutagenesis was sent for sequencing and the result showed that was perfect. A midi for germline transformation was made using QIAGEN Plasmid Plus Midi Kit (QIAGEN GmbH, 40724 Hilden, Germany).

\section{Germline transformation procedure:}

The repair template PCR-LHA-attP-Tc007675(mRNA)-P2A-tTA-3'UTR-3xP3_Tc'V_SV40attP-RHA(Fig 1) constructs, Cas9 (Tc-hsp68-Cas9 plasmid) and Guide 3 and 5 (Table 2, $p$ (U6b-BsaI-gRNA) ${ }^{28}$ ) plasmids were injected using $400 \mathrm{ng} / \mu 1,400 \mathrm{ng} / \mu \mathrm{l}$ and $250 \mathrm{ng} / \mu \mathrm{l}$ each of the guides respectively into $0-2$ hours old embryos of the vermilion ${ }^{\text {white }}\left(\mathrm{v}^{w}\right)$ strain. Borosilicate capillaries were pulled with P-2000 micropipette puller (Sutter Instrument, Novato USA). The needles were opened using a beveller (Bachhofer Laboratoriumsgeräte; Reutlingen, Germany).

After injection, the embryos were transferred onto an apple agar plates and sealed in a plastic box which was kept in an incubator set at $32^{\circ} \mathrm{C}$ for 2 days. After 2 days, the lid of the box containing the injected embryos were opened and kept until hatching ${ }^{36} 37$. Hatched larvae were picked with a hair thin brush individually and placed on full wheat flour for further 3 weeks until they were pupae. The pupae $\left(\mathrm{G}_{\mathrm{o}}\right)$ are sexed and crossed to $3 \mathrm{v}^{w}$ wildtype animals of the opposite sex.

Potential CRISPR-Cas9 transgenic individuals were screened for vermillion eye marker using cold light on a microscope equipped with camera Q imaging micropublisher 5.0 RTV (Leica Mikrosysteme Vertrieb Gmb, Wetzlar, 35578 Germany). 


\section{Characterization of the transgenic line:}

Genomic DNA was obtained as described above from the single generated line. Several $50 \mu 1$ PCR reactions were conducted to amplify different genomic regions of the CRISPR-Cas transgenic driver line (Fig 2 and Table: 3). Mixtures consisting of water $28 \mu 1$, gDNA $2 \mu 1,5 x$ Phusion buffer $10 \mu 1$, Phusion polymerase $1 \mu 1$, dNTPs $5 \mu 1$, forward primer $2.5 \mu 1$ and reverse primer $2.5 \mu 1$ (Table: 3) in a PCR program of $2 \mathrm{~min}$. at $98^{\circ} \mathrm{C}, 30 \mathrm{sec}$. at $98^{\circ} \mathrm{C}, 30 \mathrm{sec}$. at varying temperatures, $2 \mathrm{~min}$. at $72^{\circ} \mathrm{C} 35$ cycles, $10 \mathrm{~min}$. at $72^{\circ} \mathrm{C}$ were conducted in a thermocycler. The amplified fragments were run on $1 \%$ agarose gel and the distinct single bands were excised, purified by using standard practice and measured using Nanodrop 2000 Spectrophotometer (Thermo Scientific ${ }^{\mathrm{TM}}$ USA).

The blunt PCR amplified fragments obtained were cloned into Pjet 1.2 vector (Thermo Fisher Scientific, 64293 Darmstadt, Germany), using the following ligation procedure, Peg $40001 \mu 1$, T4 DNA ligase (NEB) 1 $\mu 1,10 x$ T4 DNA ligase (NEB) buffer $2 \mu 1$, Pjet 1.2 vector $(50 \mathrm{ng} / \mu \mathrm{l})$ $1 \mu 1,10 \mathrm{mM}$ ATP $2 \mu \mathrm{l}$, and varying volume of fragment based on their concentration plus water to $20 \mu \mathrm{l}$. The mixture was kept in the $16^{\circ} \mathrm{C}$ in a water bath overnight and afterwards $5 \mu 1$ of the mix was transformed into DH5 $\alpha$ competent cells and spread on pre-dried x-gal spread agar plates.

Positive clones obtained after colony PCR using standard primers pJet1.2_fwd and pJet1.2_rev was inoculated and purified by using standard practice and measured using Nanodrop 2000 Spectrophotometer (Thermo Scientific ${ }^{\mathrm{TM}}$ USA), and subsequently sequenced (Seqlab ${ }^{25}$ ).

\section{Generation of EGFP construct for phiC31-based Recombinase Mediated Cassette Exchange (RMCE).}

To generate PCRII-attB-3xP3_EGFP_SV40-attB, that will be used for the replacing the complete bi-cistronic driver construct from the genome of the generated CRISPR-Cas9 driver strain by Recombinase Mediated Cassette Exchange (RMCE). I amplified 3xP3_EGFP_SV40 from $p B a c$ \{3xP3_EGFP_SV40_af ${ }^{38}$ in a 50 $\mu 1$ reaction consisting of $28 \mu 1$ water, 5x Phusion buffer $10 \mu 1$, dNTPs $4 \mu 1,100 \mathrm{ng} / \mu 1$ of plasmid $2 \mu 1,2.5 \mu 1$ forward and reverse primers each and $1 \mu 1$ Phusion polymerase, with PCR condition $98^{\circ} \mathrm{C} 2 \mathrm{~min}$. followed by 35 cycles of $98^{\circ} \mathrm{C} 30$ sec. $67^{\circ} \mathrm{C} 30 \mathrm{sec}$ annealing temperature, and $72^{\circ} \mathrm{C} 1 \mathrm{~min}$ with a final elongation $72^{\circ} \mathrm{C}$ for 10 min. The amplified fragment 3xP3_EGFP_SV40 as well as PCRII-attB plasmid (generated by 
Ahmed Hassan Mohammed Mutasim) were cut with SpeI/EcoRI restriction enzymes in a 50 $\mu 1$ reaction. The reactions were independently run on $1 \%$ agarose and the distinct bands obtain were excise, purified and measure using standard purification procedure and Nanodrop 2000 Spectrophotometer (Thermo Scientific ${ }^{\mathrm{TM}}$ USA) respectively. The bands were subsequently ligated together to generate PCRII-attB-3xP3_EGFP_SV40 (Fig. 5) construct.

A pair of complimentary attB oligonucleotides sequence was synthesized, annealed in a $100 \mu \mathrm{l}$ reaction in the following concentration $90 \mu \mathrm{l}$ water, $5 \mu \mathrm{l}$ of the forward and reverse $10 \mathrm{mM}$ primers. The reaction was allowed to stand in a heat block set at $95^{\circ} \mathrm{C}$ for $5 \mathrm{~min}$, after which the heat block was switched off and allowed to cool down to room temperature.

The generated PCRII-attB-3xP3_EGFP_SV40 (Fig 5) plasmid was digested with SpeI and EcoRI enzymes downstream of the SV40 of the PCRII-attB-3xP3_EGFP_SV40 (Fig. 3) plasmid. The linearized plasmid was then ligated with the annealed attB oligos, thus, generating PCRII-attB-3xP3_EGFP_SV40-attB (Fig 5) construct.

\section{Generation and injection of potential mutation template for Recombinase Mediated Cassette Exchange (RMCE):}

The generated PCRII-attB-3xP3_EGFP_SV40-attB (Fig 5) plasmid was injected into 0-24 hours old embryos of the CRISPR-Cas9 early embryonic driver line to cause RMCE. The injection was done with PCRII-attB-3xP3_EGFP_SV40-attB (Fig 5) plasmid and hsp70phiC31-nls integrase (HMMA098: generated by Ahmed Hassan Mohammed Mutasim) at the concentration of $500 \mathrm{ng} / \mu \mathrm{l}$ and $300 \mathrm{ng} / \mu \mathrm{l}$ respectively. The $\mathrm{G}_{0}$ individuals were sexed at pupal stage and crossed individually to 3 wildtype individuals of the opposite sex until adult emergence.

Screening for potential transgenic beetles with expected EGFP expression in the eyes was done using LEICA M205 FA stereomicroscope epifluorescence microscope with the filter EGFPLP (excitation: ET480/40, emission: ET510 LP).

\section{Whole Mount In-Situ Hybridization (WMISH):}

WMISH was conducted on 0-24 hours old embryos using DIG-labelled anti sense RNA probe. Probes were synthesized by utilizing the DIG-RNA-Labelling Kit (Roche, Mannheim). Staining for expression was perform using NBT/ BCIP as described ${ }^{39}$. 


\section{Microscopy:}

Image acquisition with cy3 filter, 8bit mono and black/white, and also DIC filter, 24bit color and RGB settings of Zeiss Axioplan 2 microscope for in situ pictures. All figures were prepared using Adobe Photoshop (CS6)

\section{DISCUSSION:}

The discovery of the DNA as a unit of heredity ${ }^{42}$ and as a dogma of the molecular biology, led to the understanding of DNA to RNA and protein relationship. This also provided molecular biologist with the ability to investigate the various functions of genes through genetic engineering ${ }^{43}{ }^{44}$ hence leading to the achievement of several feats in pest ${ }^{45}$ and disease ${ }^{45}$ management, functions of other genes, epigenetic studies ${ }^{46} 47$, gene therapy ${ }^{48}$ among others. Also, the understanding that foreign DNA can be stably integrated into the genome of other organisms to modify it, has provided many opportunities for biomedical and biological fields. There are various forms of genetic modification ${ }^{44,49}$ techniques that can be employ to the genome of several organisms for deletion of certain DNA sequences, knock in some specific DNA sequences and replacement of certain DNA sequences with foreign or modified DNA sequences. The different types of genetic modification techniques used for the insertion/deletion or replacement of DNA sequences of an organism, include germline transformation using piggyBac transformation, Sleeping Beauty ${ }^{50}$, or gene editing technologies such as TALEN ${ }^{51}$, CRISPR-Cas $9^{3}$ etc.

CRISPR-Cas $9^{3}$ is a RNA-guided nucleases that is use to degrade any invading foreign genetic materials, or use to modify the genome of an organism to insert any gene of interest. CRISPRCas9 genome editing has recently been used over wide clade of animal fauna, such as dipteran and some coleopteran. Though there is a lot of data showing the effectiveness of the technique in dipteran insect pest, but however with a paucity of information for coleopteran insects. Tribolium castaneum ${ }^{52-54}$ is an important model organism in the order coleoptera, which have been employed in the study of gene function, and for both evolutionary and developmental biology question for pest control measures in a wide range Integrated Pest Management (IPM) practices.

We use the CRISPR-Cas system to generate a driver line by modifying the Tc007675 gene locus which is expressed at the early embryonic cellularization stage. The driver line can be 
used in a heterologous transactivator binary expression system ${ }^{55-57}$ which could result in the establishment of conditional sexing ${ }^{16,19,32,58-60}$ and embryonic lethality 20,37,61 systems subsequently based on the tTA expression as we observed. Using Recombinase Mediated Cassette Exchange (RMCE) 10,13, , we aimed to create mutants for the Tc007675 early embryonic cellularization specific genes.

Thus, using the techniques we were able to successfully edit the genome of Tribolium casteneum using the Homology Directed Repair (HDR) cell repair mechanism to generate a tTA driver line in the locus of the new discovered early cellularization embryonic gene Tc007675. Though several efforts have being employed in our lab by several scientist to use CRISPR-Cas9 genome editing technique on the tribolium with no success (Personal communication).

Generation of mutant with null allele is one of the standard techniques that is used to evaluate the function of a gene aside other practices such as RNAi. Thus, having generated the driver line using the locus of the newly discovered Tc007675 early embryonic gene to drive tTA by HDR, we aim to generate mutants for the Tc007675 using RMCE ${ }^{9,13}$ system that will give us the opportunity to investigate the function of the novel gene in-situ.

We are, however, not able to generate mutants for further downstream experiment for now, which could be due to the drosophila $h s p 70$ basal promoter used for mediating the phic31-nlsintegrase. To repeat the experiment, I will replace the drosophila $h s p 70$ basal promoter with the T. casteneum hsp68 basal promoter to mediate the phic31-nls-integrase. 


\section{REFERENCES}

1. Barrangou, R. \& Marraffini, L. A. CRISPR-Cas systems: prokaryotes upgrade to adaptive immunity. Mol Cell 54, 234-244 (2014).

2. Sander, J. D. \& Joung, J. K. CRISPR-Cas systems for genome editing, regulation and targeting. Nat Biotechnol 32, 347-355 (2014).

3. CRISPR Cas System - an overview | ScienceDirect Topics. https://www.sciencedirect.com/topics/neuroscience/crispr-cas-system.

4. Gu, J. \& Lieber, M. R. Mechanistic flexibility as a conserved theme across 3 billion years of nonhomologous DNA end-joining. Genes Dev. 22, 411-415 (2008).

5. Bibikova, M., Golic, M., Golic, K. G. \& Carroll, D. Targeted chromosomal cleavage and mutagenesis in Drosophila using zinc-finger nucleases. Genetics 161, 1169-1175 (2002).

6. Liang, F., Han, M., Romanienko, P. J. \& Jasin, M. Homology-directed repair is a major doublestrand break repair pathway in mammalian cells. PNAS 95, 5172-5177 (1998).

7. Belteki, G., Gertsenstein, M., Ow, D. W. \& Nagy, A. Site-specific cassette exchange and germline transmission with mouse ES cells expressing phiC31 integrase. Nat. Biotechnol. 21, 321-324 (2003).

8. Nimmo, D. D., Alphey, L., Meredith, J. M. \& Eggleston, P. High efficiency site-specific genetic engineering of the mosquito genome. Insect Mol Biol 15, 129-136 (2006).

9. Sauer, B. \& Henderson, N. Site-specific DNA recombination in mammalian cells by the Cre recombinase of bacteriophage P1. Proc. Natl. Acad. Sci. U.S.A. 85, 5166-5170 (1988).

10. Thorpe, H. M. \& Smith, M. C. In vitro site-specific integration of bacteriophage DNA catalyzed by a recombinase of the resolvase/invertase family. Proc. Natl. Acad. Sci. U.S.A. 95, 5505-5510 (1998).

11. Haghighat-Khah, R. E. et al. Site-specific cassette exchange systems in the Aedes aegypti mosquito and the Plutella xylostella moth. PLOS ONE 10, e0121097 (2015). 
12. Baer, A. \& Bode, J. Coping with kinetic and thermodynamic barriers: RMCE, an efficient strategy for the targeted integration of transgenes. Curr Opin Biotechnol 12, 473-480 (2001).

13. O'Gorman, S., Fox, D. T. \& Wahl, G. M. Recombinase-mediated gene activation and site-specific integration in mammalian cells. Science 251, 1351-1355 (1991).

14. Wimmer, E. A. Insect transgenesis by site-specific recombination. Nature Methods 2, 580-582 (2005).

15. Schetelig, M. F., Wimmer, E. A., Scolari, F., Gasperi, G. \& Handler, E. A. New genetic tools for improving SIT in Ceratitis capitata: embryonic lethality and sperm marking.

16. Ogaugwu, C. E., Schetelig, M. F. \& Wimmer, E. A. Transgenic sexing system for Ceratitis capitata (Diptera: Tephritidae) based on female-specific embryonic lethality. Insect Biochemistry and Molecular Biology 43, 1-8 (2013).

17. Zhang, C. X., Lee, M. P., Chen, A. D., Brown, S. D. \& Hsieh, T. Isolation and characterization of a Drosophila gene essential for early embryonic development and formation of cortical cleavage furrows. J Cell Biol 134, 923-934 (1996).

18. Alphey, L. et al. Genetic control of Aedes mosquitoes. Pathog Glob Health 107, 170-179 (2013).

19. Schetelig, M. \& Handler, A. A transgenic embryonic sexing system for Anastrepha suspensa (Diptera: Tephritidae). Insect biochemistry and molecular biology 42, 790-5 (2012).

20. Schetelig, M. F., Caceres, C., Zacharopoulou, A., Franz, G. \& Wimmer, E. A. Conditional embryonic lethality to improve the sterile insect technique in Ceratitis capitata(Diptera: Tephritidae). BMC Biol 7, 4 (2009).

21. Heinrich, J. C. \& Scott, M. J. A repressible female-specific lethal genetic system for making transgenic insect strains suitable for a sterile-release program. Proc Natl Acad Sci U S A 97, $8229-8232$ (2000).

22. Tan, A. et al. Transgene-based, female-specific lethality system for genetic sexing of the silkworm, Bombyx mori. PNAS 110, 6766-6770 (2013). 
23. Lorenzen, M., Brown, S., Denell, R. \& Beeman, R. Cloning and Characterization of the Tribolium castaneum Eye-Color Genes Encoding Tryptophan Oxygenase and Kynurenine 3Monooxygenase. Genetics 160, 225-34 (2002).

24. Liu, H. \& Naismith, J. H. An efficient one-step site-directed deletion, insertion, single and multiple-site plasmid mutagenesis protocol. BMC Biotechnol 8, 91 (2008).

25. Microsynth Webshop. https://srvweb.microsynth.ch/.

26. Gratz, S. J. et al. Highly specific and efficient CRISPR/Cas9-catalyzed homology-directed repair in Drosophila. Genetics 196, 961-971 (2014).

27. iBeetle. http://ibeetle-base.uni-goettingen.de/.

28. Gilles, A. F., Schinko, J. B. \& Averof, M. Efficient CRISPR-mediated gene targeting and transgene replacement in the beetle Tribolium castaneum. Development 142, 2832-2839 (2015).

29. Ahmed, H. M. M., Hildebrand, L. \& Wimmer, E. A. Improvement and use of CRISPR/Cas9 to engineer a sperm-marking strain for the invasive fruit pest Drosophila suzukii. BMC Biotechnol 19, 85 (2019).

30. T7 endo I assay - CRISPR fly design. https://www.crisprflydesign.org/t7-endo-i-assay/.

31. Sentmanat, M. F., Peters, S. T., Florian, C. P., Connelly, J. P. \& Pruett-Miller, S. M. A Survey of Validation Strategies for CRISPR-Cas9 Editing. Scientific Reports 8, 888 (2018).

32. Eckermann, K. N. et al. Hyperactive piggyBac transposase improves transformation efficiency in diverse insect species. Insect Biochemistry and Molecular Biology 98, 16-24 (2018).

33. Biolabs, N. E. Determining Genome Targeting Efficiency using T7 Endonuclease I| NEB. https://international.neb.com/protocols/2014/08/11/determining-genome-targetingefficiency-using-t7-endonuclease-i.

34. Biolabs, N. E. NEBuilder Hifi DNA Assembly Reaction Protocol | NEB. https://international.neb.com/protocols/2014/11/26/nebuilder-hifi-dna-assembly-reactionprotocol. 
35. Potapov, V. et al. Comprehensive Profiling of Four Base Overhang Ligation Fidelity by T4 DNA Ligase and Application to DNA Assembly. ACS Synth. Biol. 7, 2665-2674 (2018).

36. Sj, B. et al. The Red Flour Beetle, Tribolium Castaneum (Coleoptera): A Model for Studies of Development and Pest Biology. Cold Spring Harbor protocols https://pubmed.ncbi.nlm.nih.gov/20147228/ (2009) doi:10.1101/pdb.emo126.

37. Trauner, J. et al. Large-scale insertional mutagenesis of a coleopteran stored grain pest, the red flour beetle Tribolium castaneum, identifies embryonic lethal mutations and enhancer traps. BMC Biol 7, 73 (2009).

38. Horn, C. \& Wimmer, E. A versatile vector set for animal transgenesis. Development genes and evolution 210, 630-7 (2001).

39. Schinko, J., Posnien, N., Kittelmann, S., Koniszewski, N. \& Bucher, G. Single and Double WholeMount In Situ Hybridization in Red Flour Beetle (Tribolium) Embryos. Cold Spring Harbor Protocols 2009, pdb.prot5258-pdb.prot5258 (2009).

40. Oberhofer, G., Grossmann, D., Siemanowski, J. L., Beissbarth, T. \& Bucher, G. Wnt/ $\beta$-catenin signaling integrates patterning and metabolism of the insect growth zone. Development 141, 4740-4750 (2014).

41. Siemanowski, J., Richter, T., Dao, V. A. \& Bucher, G. Notch signaling induces cell proliferation in the labrum in a regulatory network different from the thoracic legs. Dev. Biol. 408, 164-177 (2015).

42. McCarty, M. Discovering genes are made of DNA. Nature 421, 406-406 (2003).

43. Park, M. A., Jung, H. S. \& Slukvin, I. Genetic Engineering of Human Pluripotent Stem Cells Using PiggyBac Transposon System. Curr Protoc Stem Cell Biol 47, e63 (2018).

44. Laptev, I. A., Raevskaya, N. M., Filimonova, N. A. \& Sineoky, S. P. The piggyBac Transposon as a Tool in Genetic Engineering. Appl Biochem Microbiol 53, 874-881 (2017).

45. Vincelli, P. Genetic Engineering and Sustainable Crop Disease Management: Opportunities for Case-by-Case Decision-Making. Sustainability 8, 495 (2016). 
46. Moosavi, A. \& Ardekani, A. M. Role of Epigenetics in Biology and Human Diseases. Iran Biomed J 20, 246-258 (2016).

47. Magnani, L. Epigenetic engineering and the art of epigenetic manipulation. Genome Biology 15, 306 (2014).

48. Doerfler, W. Epigenetic consequences of genome manipulations: caveats for human germline therapy and genetically modified organisms. Epigenomics 11, 247-250 (2019).

49. Wimmer, E. A. Applications of insect transgenesis. Nat Rev Genet 4, 225-232 (2003).

50. Izsvák, Z. \& Ivics, Z. Sleeping Beauty Transposition: Biology and Applications for Molecular Therapy. Molecular Therapy 9, 147-156 (2004).

51. Nemudryi, A. A., Valetdinova, K. R., Medvedev, S. P. \& Zakian, S. M. TALEN and CRISPR/Cas Genome Editing Systems: Tools of Discovery. Acta Naturae 6, 19-40 (2014).

52. Brown, S. J. et al. The Red Flour Beetle, Tribolium castaneum (Coleoptera): A Model for Studies of Development and Pest Biology. Cold Spring Harbor Protocols 2009, pdb.emo126pdb.emo126 (2009).

53. Kumar, H. et al. Red flour beetle (Tribolium castaneum): From population genetics to functional genomics. Vet World 11, 1043-1046 (2018).

54. Richards, S. et al. The genome of the model beetle and pest Tribolium castaneum. Nature $\mathbf{4 5 2}$, 949-955 (2008).

55. Das, A. T., Tenenbaum, L. \& Berkhout, B. Tet-On Systems For Doxycycline-inducible Gene Expression. Curr Gene Ther 16, 156-167 (2016).

56. Gossen, M. \& Bujard, H. Tight control of gene expression in mammalian cells by tetracyclineresponsive promoters. Proc. Natl. Acad. Sci. U.S.A. 89, 5547-5551 (1992).

57. Bello, B., Resendez-Perez, D. \& Gehring, W. J. Spatial and temporal targeting of gene expression in Drosophila by means of a tetracycline-dependent transactivator system. 10.

58. Catteruccia, F., Benton, J. P. \& Crisanti, A. An Anopheles transgenic sexing strain for vector control. Nature Biotechnology 23, 1414-1417 (2005). 
59. Meza, J. S. et al. Comparison of classical and transgenic genetic sexing strains of Mediterranean fruit fly (Diptera: Tephritidae) for application of the sterile insect technique. PLoS One 13, (2018).

60. Rendón, P., Mclnnis, D., Lance, D. \& Stewart, J. Medfly (Diptera: Tephritidae) genetic sexing: large-scale field comparison of males-only and bisexual sterile fly releases in Guatemala. J. Econ. Entomol. 97, 1547-1553 (2004).

61. Horn, C. \& Wimmer, E. A. A transgene-based, embryo-specific lethality system for insect pest management. Nature Biotechnology 21, 64-70 (2003). 


\section{FIGURES}

A)

\begin{tabular}{|l|l|l|l|l|l|}
\hline LHA & EXON II & $3^{\prime}$ UTR & RHA $^{\star}$ \\
\hline
\end{tabular}

B)

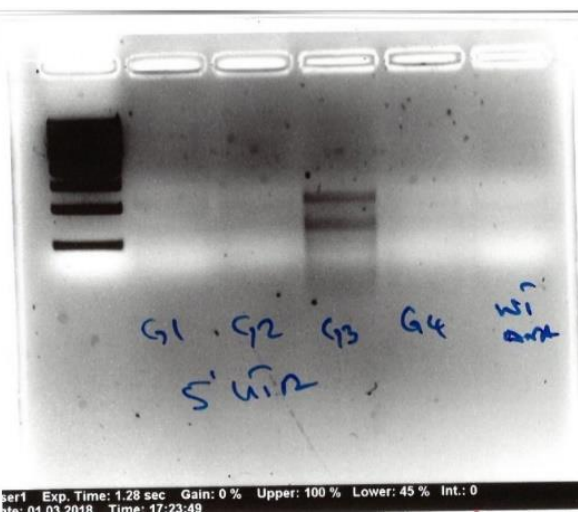

Target sequence 5 - 3'

g3 = TTCGGTAGGTATGGGACAGTTAG
C)

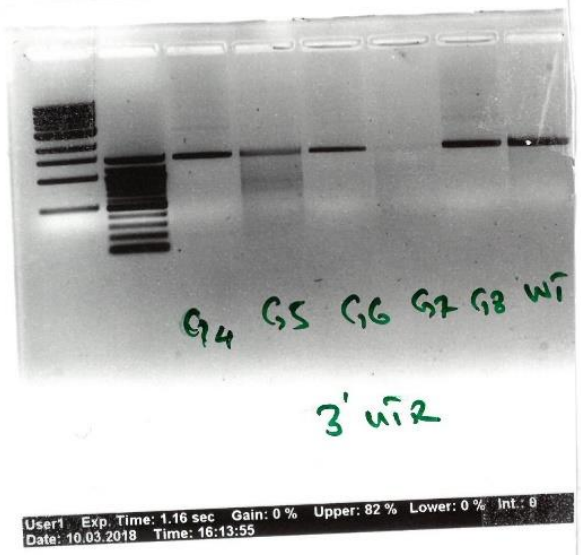

Target sequence 5 - $3^{\prime}$

g5 = TGGATTGGGTCGTTAAGCGTAGG

Fig 1: Identification and validation of 2 guide RNAs used for HDR A) structure of the gene locus of Tc007675 and several guides were tested with 2 guides, one each for the LHA and RHA found to be effective. B) agarose gel pictures depicting the efficiency of the gene when tested by T7 endonuclease assay C) sequence of each target with the pam shown in red. 


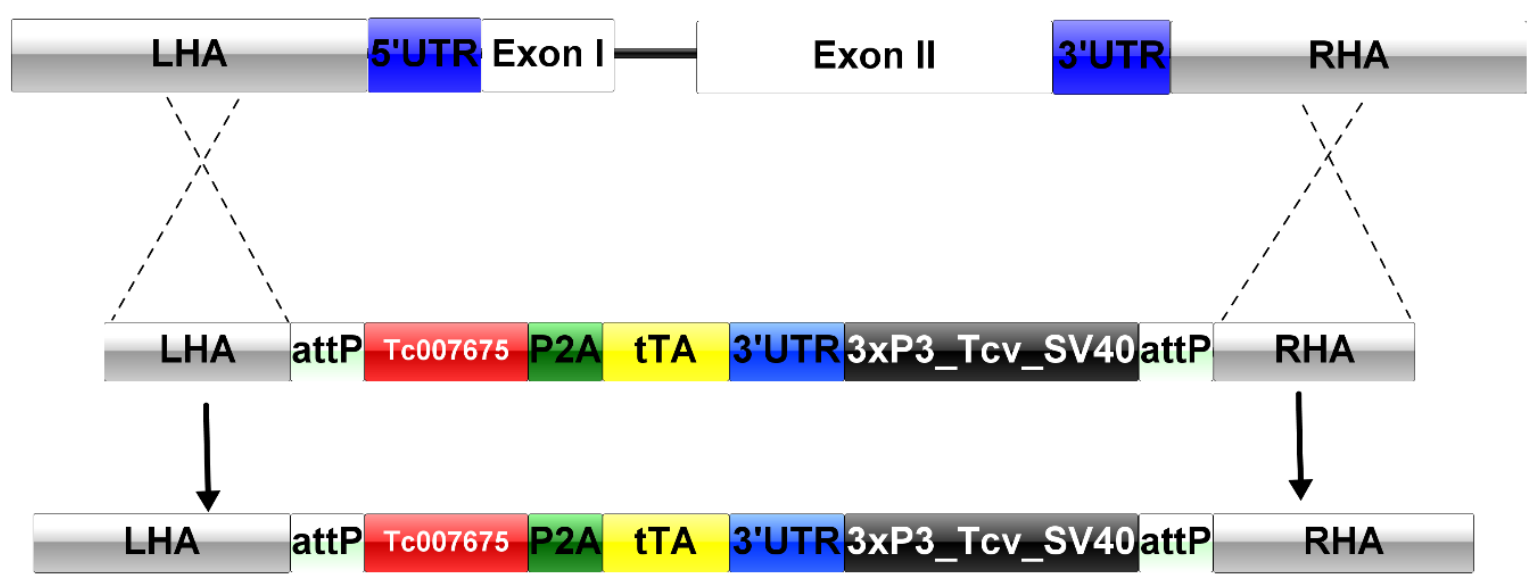

Fig 2: A schematic depiction for the cloning of driver strain construct used for generation of PCR-LHA-attP-Tc007675 (mRNA)-P2A-tTA, and D) PCR-LHA-attP-Tc007675 (mRNA)P2A-tTA-3'UTR-3xP3_Tc'V_SV40-attP-RHA construct for CRISPR/Cas9 germline mediated driver line.

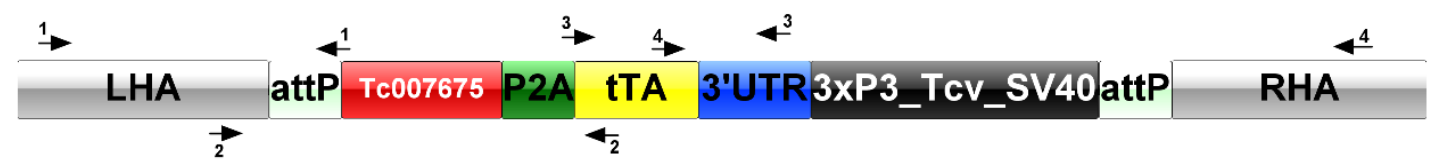

Fig 3: Schematic depiction of the driver construct used and the also showing the positions of the primers used for the characterization of the CRISPR/Cas9 driver line. 


\section{Dig Staining Dapi Staining}
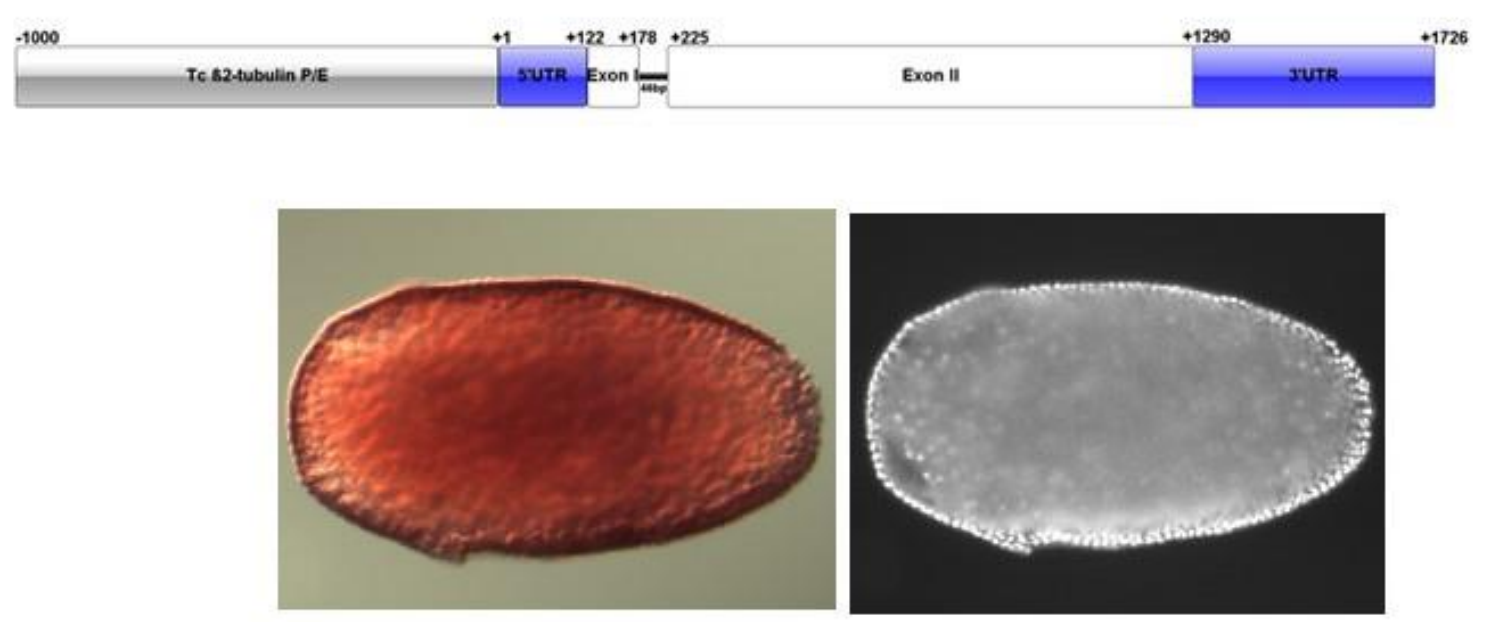

\section{LHA attP Tc007675 P2A tTA 3 3'UTR3XP3_Tcv_SV40 attP $\quad$ RHA}
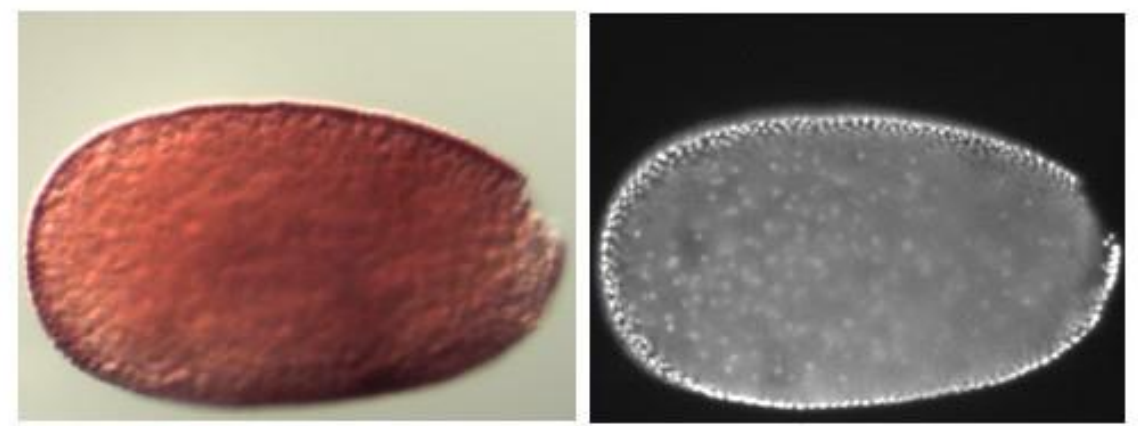

Fig 4: Whole Mount in Situ Hybridization of wildtype and CRISPR/Cas9 germline mediated Tc007675 embryonic specific driver line embryos. 


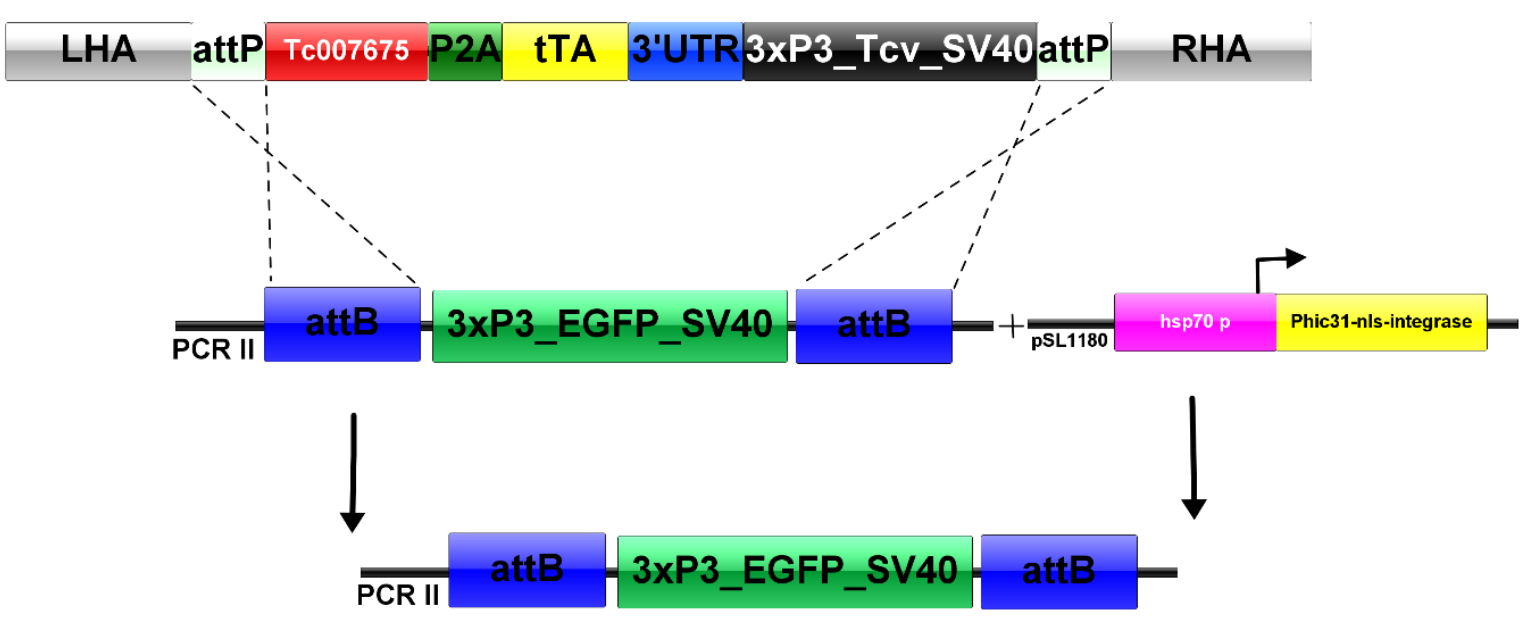

Fig 5: A schematic step by step cloning of RMCE repair template for the generation of Tc007675 mutants: 
Table 1: List of primer used in this experiment

\begin{tabular}{|c|c|c|}
\hline ID & NAME & Sequence 5' - 3' \\
\hline MID\#178 & XbaI-BbsI-P2A-XbaI-F & $\begin{array}{l}\text { CTAGAGAAGACGGGTCCGGCG...AGGAGA } \\
\end{array}$ \\
\hline MID\#179 & XbaI-P2A-BbsI-Rv & CTAGACATGGGGCCGGGGTTC...GTGGCG \\
\hline MID\#180 & RHA-fwd & GCGACTAGTACAGTAATTTTTTTTTTGATT \\
\hline MID\#181 & RHA-rev & $\begin{array}{l}\text { TGCATCAAGCTTGGTACCGAGCTCGGCTCT } \\
\end{array}$ \\
\hline MID\#182 & 3xP3-TcV-attP-RHA-f & GTCCAAACTCATCACTAGTACTGACGGACA \\
\hline MID\#183 & 3xP3-TcV-attP-RHA-r & ATCAAAAAAAAAATTACTGTACTAGTCGCG \\
\hline MID\#184 & $3 \times \mathrm{x} 3-\mathrm{TcV}-\mathrm{fwd}$ & $\begin{array}{l}\text { GATTTTGACATAATAATTCAATTAGAGACT } \\
\end{array}$ \\
\hline MID\#185 & 3xP3-TcV-rev & CGTCAGTACTAGTGATGAGTTTGGACAAAC \\
\hline MID\#186 & 3'UTR-fwd-HIFI-2 & GAGTACGGTGGGTAGGGGGCG...AATAAT \\
\hline MID\#187 & 3'UTR-rev-HIFI-2 & $\begin{array}{l}\text { TCTAATTGAATTATTATGTCAAAATCATTT } \\
\end{array}$ \\
\hline MID\#188 & TC007675-P2A-tTA-fwd & GCGACTAGTACAGACTTGTTGTTTAAACCC \\
\hline MID\#189 & TC007675-P2A-tTA-REV & TCAGCAGGGAGAAGTTGGTGGCGCCGGACC \\
\hline MID\#190 & LHA-attP-TC007675-fwd & AGCACCCCTAATCACTAGTACTGACGGACA \\
\hline MID\#191 & $\begin{array}{l}\text { LHA-attP-TC007675- } \\
\text { REV }\end{array}$ & TTAAACAACAAGTCTGTACTAGTCGCGCTC \\
\hline MID\#192 & LHA-fwd-HIFI-1 & GAATTGGGCCCTCTAGAGAAG...GATAAC \\
\hline MID\#193 & LHA-REV-HIFI-1 & $\begin{array}{l}\text { CGTCAGTACTAGTGATTAGGGGTGCTAAAG } \\
\end{array}$ \\
\hline MID\#194 & RHA_Mut_G5_3UTR_F & TCTACGCTTAACGACCCAATCCAATTTCTAAACAT \\
\hline MID\#195 & RHA_Mut_G5_3UTR_R & TGGATTGGGTCGTTAAGCGTAGAATGCTGACTTCATTAG \\
\hline MID\#196 & LHA_Mut_G3_5UTR_F & $\begin{array}{l}\text { ATCCTAACTGTCCCATACCTACCTGCCTATAAATAC } \\
\end{array}$ \\
\hline MID\#197 & LHA_Mut_G3_5UTR_R & $\begin{array}{l}\text { GTAGGTATGGGACAGTTAGGATTGGTGGAAGGG } \\
\end{array}$ \\
\hline MID\#198 & Phi-int-3xP3-F & AGATCGAATTCATTATTCATTAGAGACTAATTCAATTAGAGCTAATTCAATTAGG \\
\hline MID\#199 & Phi-int-SV40-R & AGATCGAATTCGATGAGTTTGGACAAACCACAACTAGAATGCAGTG \\
\hline MID\#200 & Phi-int-attB-F & GTGAGGTGGAGTACGCGCCCGGGGAG \\
\hline \multirow[t]{4}{*}{ MID\#201 } & Phi-int- attB -R & TGCGGGTGCCAGGGCGTGCCCTTG \\
\hline & Pjet 1.2 Forward & CGACTCACTATAGGGAGAGCGGC \\
\hline & Pjet 1.2 Reverse & AAGAACATCGATTTTCCATGGCAG \\
\hline & Pjet 1.2 Forward_T7 & $\begin{array}{l}\text { GAATTGTAATACGACTCACTATAGGCGA CTCACTATAGGGAGAGC } \\
\end{array}$ \\
\hline
\end{tabular}

Table 2: list of guide oligos used for the experiment

\begin{tabular}{|l|l|l|}
\hline ID & Guide Oligo name & Sequence 5' - 3' \\
\hline MID\#139 & 3'-G4-Fwd-007675 & TTCGTGGATTGGGTCGTTAAGCGT \\
\hline MID\#140 & 3'-G4-Rev-007675 & AAACACGCTTAACGACCCAATCCA \\
\hline MID\#141 & 3'-G5-Fwd-007675 & TGGATTGGGTCGTTAAGCGTAGG \\
\hline MID\#142 & 3'-G5-Rev-007675 & ACGCTTAACGACCCAATCCATCC \\
\hline MID\#141 & 3'-G6-Fwd-007675 & TTCGAATGAAGTCAGCATCCTACG \\
\hline MID\#142 & 3'-G6-Rev-007675 & AAACAATGAAGTCAGCATCCTACG \\
\hline MID\#143 & 3'-G7-Fwd-007675 & TTCGAAGAGAGTACAGGTTGGTA \\
\hline MID\#144 & 3'-G7-Rev-007675 & AAACTACCAACCTGTACTCTCTT \\
\hline MID\#145 & 3'-G8-Fwd-007675 & TTCGTTTTCTTGATTCCGAAGGC \\
\hline MID\#146 & 3'-G8-Rev-007675 & AAACGACTTCGGAATCAAGAAAA \\
\hline MID\#147 & 3'-G9-Fwd-007675 & TTCGTCTCTTTTTTATGTGCGAG \\
\hline MID\#148 & 3'-G9-Rev-007675 & AAACCTCGCACATAAAAAAGAGA \\
\hline MID\#149 & 5'-G1-Fwd-007675 & TTCGGGAGTATTTATAGGCAGGT \\
\hline MID\#150 & 5'-G1-Rev-007675 & AAACACCTGCCTATAAATACTCC \\
\hline MID\#151 & 5'-G2-Fwd-007675 & TTCGGACAGTTAGGGGTGGTGGA \\
\hline MID\#152 & 5'-G2-Rev-007675 & AAACTCCACCACCCCTAACTGTC \\
\hline MID\#153 & 5'-G3-Fwd-007675 & TTCGGTAGGTATGGGACAGTTAG \\
\hline MID\#154 & 5'-G3-Rev-007675 & AAACCTAACTGTCCCATACCTAC \\
\hline MID\#155 & 5'-G4-Fwd-007675 & TTCGACAGTTAGGGGTGGTGGAA \\
\hline MID\#156 & 5'-G4-Rev-007675 & AAACTTCCACCACCCCTAACTGT \\
\hline
\end{tabular}


Table 3: Showing the primer used, sequences, annealing temperature and fragment sized obtained for the characterization of CRISPR-Cas9 generated line

\begin{tabular}{|c|c|c|c|c|}
\hline ID & NAME & Sequence 5'-3' & $\begin{array}{l}\text { Annealing } \\
\text { temperature }\end{array}$ & $\begin{array}{l}\text { Size of } \\
\text { amplified } \\
\text { fragment }\end{array}$ \\
\hline MID\#130 & TC007675-int-R & GCGACTGCCCACATCGTAATTG & \multirow[t]{2}{*}{72} & \multirow[t]{2}{*}{$3000 \mathrm{bp}$} \\
\hline MID\#134 & tTA-Ends-Seq & CGGCCTTGAATTGATCATATGCGG & & \\
\hline MID\#118 & TC007675-F-1.4-Cla1 & $\begin{array}{l}\text { GCCGATCGATTAAACAACAAGTG } \\
\end{array}$ & \multirow[t]{2}{*}{61} & \multirow[t]{2}{*}{$1600 \mathrm{bp}$} \\
\hline MID\#191 & $\begin{array}{l}\text { LHA-attP-TC007675- } \\
\text { REV }\end{array}$ & TTAAACAACAAGTCTGTACTAGTCGCGCTC & & \\
\hline MID\#196 & LHA_Mut_G3_5UTR_F & ATCCTAACTGTCCCATACCTACCTGCCTATAAATAC & \multirow[t]{2}{*}{72} & \multirow[t]{2}{*}{$900 \mathrm{bp}$} \\
\hline MID\#189 & $\begin{array}{l}\text { TC007675-P2A-tTA- } \\
\text { REV }\end{array}$ & TCAGCAGGGAGAAGTTGGTGGCGCCGGACC & & \\
\hline MID\#188 & $\begin{array}{l}\text { TC007675-P2A-tTA- } \\
\text { fwd }\end{array}$ & GCGACTAGTACAGACTTGTTGTTTAAACCC & \multirow[t]{2}{*}{64} & \multirow[t]{2}{*}{ 1700bp } \\
\hline MID\#187 & 3'UTR-rev-HIFI-2 & TCTAATTGAATTATTATGTCAAAATCATTT & & \\
\hline
\end{tabular}




\section{General discussion:}

\subsection{Transgenic sperm marking in Tribolium castaneum}

Transgenic sperm marking ${ }^{126,152}$ is achieved with the utilization of the spermatogenesis specific B2t Promoter/Enhancer (P/E) ${ }^{144,153,154}$ region to drive either DsRed ${ }^{155}$ or EGFP 156 with the intention of marking the sperm of generated transgenic males. However, before the used of biotechnological approach for sperm marking, males made sterile with irradiation $111,112,118$ or any suitable measure that are ready to be released are bodily dusted with fluorescent dyes to ease monitoring. This then enable the evaluation of the released individuals in a planned control measure during the monitoring. Thus, despite the success recorded in using this classical form of the approach i.e. fluorescent dye ${ }^{37}$ for monitoring, there have being several attendant and verifiable drawbacks that were observed, and among which included: i) washing off of the dyes by water such as rain since the marking is bodily surficial resulting in false negatives; ii) possibility of the dyes being rubbed off on wildtype non treated individuals when the insects come in contact with treated individuals hence resulting in false positives and iii) also there is high potential human health for the workers and environmental health risk ${ }^{112}$ due to exposure to possible leakages during treatment.

Therefore, the potential drawback of the classical approach have being perfectly overcome by the use of biotechnological transgenic system approach, were the spermatogenesis-specific gene (ß2t) Promoter/Enhancer $(\mathrm{P} / \mathrm{E})$ region is used to mediate expression of either DsRed or EGFP in the sperm of the transgenic male. This system had been efficiently used to transform the males of various dipteran insect pests such as Ceratitis capitata ${ }^{126}$, Drosophila melanogaster ${ }^{138}$, D. suzukii ${ }^{152}$, Anopheles gambiae ${ }^{157}$ but was not reported for any coleopteran insect pests aside Tribolium castaneum. The ability to successfully mark the sperm provides seamless and infallible monitoring in the competence of the released sterile males in mating and paternity success compare to wildtype male individuals in an insect pest control measure as observed in our studies.

Also, we studied the reproductive biology and usage of the sperm in twice mated females in detail by observing the storage ${ }^{158}$ and usage of sperm in the spermatheca ${ }^{159}$ and/or other parts of the reproductive organ of the female. Our studies confirm that the female utilized the sperms of the second mated male first ${ }^{160}$ probably because most of the sperms of the first mated male are stored in the bursa copulatrix and also encapsulated by the second mated male sperm. 
However, our study further shows that the female continues to use the sperms of the two males over a prolong period in different proportions.

\subsection{Identification and characterization of a potential early embryonic cellularization gene} (s) in Tribolium castaneum

The economic importance of arthropod pests especially from the class Insecta on both human and agricultural produce has being huge and well documented over the years. Yearly economic damages of quantitative and qualitative running into billions of dollars have being reported ${ }^{161}$. However, in the class insecta, the order coleopteran is the largest group consisting of the most prominent key pests causing huge economic damage. Several management strategies such as; biological ${ }^{85}$ physical ${ }^{162}$, chemical controls ${ }^{4,163}$ etc. have being employed for the management of such insect, with however many disadvantages like high cost of control, drudgery in labour, development of resistance ${ }^{164}$ due to injudicious use of chemicals ${ }^{165}$ among others have being reported.

The realization of these problems throws out a challenge for research scientist to proffer suitable, healthy and environmentally acceptable pest management solutions like adoption of a gametic approach such as the Sterile Insect Technique (SIT) ${ }^{100,110}$. SIT is an efficient induced birth management system that is used as an alternative to chemical control practices. To improve such systems by transgenic approaches, one of the requirements is the enhancers/promoter $(\mathrm{P} / \mathrm{E})$ region of a gene ${ }^{166}$ express at early embryonic developmental stage.

Several early embryonic genes were identified from dipteran insect pests which include, bottleneck ${ }^{167}$, nullo ${ }^{168}$, serendipity $\alpha^{168}$, slam etc. and have been used in several ways for the generation of transgenic individuals within the clade. However, none has been reported for a coleopteran insect pest. Thus with the drosophila gene information retrieved from the flybase database ${ }^{169}$, we did a blast search ${ }^{16,170}$ using the protein sequences of the reported dipteran early embryonic genes on the Tribolium genome browser but we could not identify any conserved ortholog.

Afterwards, we mined the transcriptomic data of tribolium early embryonic genes ${ }^{171}$ and the iBeetle database ${ }^{170}$ for the possibility of identifying any possible candidate. I identified 10 and 5 genes from the transcriptomic data and iBeetle data base, respectively. After a series of WMISH we identified the Tc007675 gene as among the first reported candidate of a cellularization gene for coleopteran insect pest. We further performed parental RNAi and 
observed the no expression of the gene in the RNAi embryos. However, no induced identifiable phenotype was observed in cuticle prep analysis or the determination of any embryonic stages

I amplified and cloned $2.1 \mathrm{~Kb}$ or $1.4 \mathrm{~Kb}$ Tc007675 $\mathrm{P} / \mathrm{E}$ of the identified gene individually in order to mediate the expression of $\mathrm{tTA}^{172}$ at early embryonic stages. Several transgenic individuals were generated by piggyBac germline transformation ${ }^{28,173,174}$ and expression of tTA determined by WMISH .

Thus, this is the first time that a potential gene has been identified and used for functional studies, and transgenic individuals have been generated by germline transformation and verified by WMISH to mediate cellularization-specific gene expression. Furthermore, we crossed the potential driver lines M63_F1 we generated to several effector embryonic lines expressing green fluorescent protein tGFP which is already in the laboratory stock to verify the ability of the driver lines to drive the tGFP in the embryo which thus will result in the expression of green fluorescent in the embryos. The result we obtained for the crosses is inconclusive and we intend to perform more experiment to validate the system.

\subsection{Female Specific Embryonic Lethality system (FSEL) in Tribolium castaneum}

The FSEL has been developed for the management of insect pest species such as Ceratitis capitata $^{134}$. However, for the system to be developed and be used efficiently and effectively for early embryonic lethality system ${ }^{124}$, several requirements are needed and these includes: Enhancer/Promoter (P/E) region of a gene (s) expressed specially during early embryonic development, tet-off binary expression system ${ }^{175}$, pro-apoptotic gene ${ }^{16,176}$, and a female specific sliced intron. Moreover, the system must be expressed in a conditional manner which is regulated by tetracycline ${ }^{135,136}$ or any other suitable repressible system ${ }^{175}$ that will act in such a way that the pro-apoptotic effector gene is transcribed at embryonic stage driven by the $\mathrm{P} / \mathrm{E}$ early embryonic gene. The use of a female-specific spliced intron will lead to correct splicing in female embryos resulting in the death of only female embryos due to expression of the effector gene which conversely does not occur in the male embryos as reported in transgenic sexing system of the agricultural invasive pest Medfly C. capitata $^{123,142,177}$.

Transgenic embryonic and/or Female Specific Embryonic Lethality (FSEL) systems ${ }^{123}$ is an important component for an AW-IPM. The system is used to cause embryonic or female specific embryonic death based on apoptosis 131,134 which makes rearing of only male transgenic individuals during embryonic development possible. Hitherto, several strategies 
were employed for the sexing of insects species which include phenotypic death induced by radiation mutagenesis ${ }^{151}$ and chemical approach ${ }^{178}$, physiological and morphological features. However, not all could be applied to all insect organisms, because they are sometimes speciesspecific due to pupal size variant and also time of emergence from the embryo, it also requires a lot of decades of hard work, efforts and the resources available are limited.

\subsection{Genetic improvement and use of CRISPR/Cas9 in Tribolium castaneum for genome editing}

CRISPR-Cas is a novel genome editing system of cleaving the DNA of invading foreign body by using nucleases that are RNA guided ${ }^{59}$. The CRISPR/Cas9 system have being employed and used among varied number of organism and systems for gene editing ranging from smaller structures like yeast and higher clade like human $57,59,63,64$. It has also been used for functional analysis through gene knockdown ${ }^{61}$, targeted mutagenesis ${ }^{179}$ gene repression ${ }^{180}$ or activation ${ }^{181}$. Also, it has been used for epigenetic imprinting by methylation or demethylation ${ }^{182}$.

CRISPR/Cas9 systems have being employed in various animal species, including insects ${ }^{183} 184$ 185. Within the class insecta the technique has been used over a wide range of insect orders to edit different genomic targets for both functional and evolutionary purposes. Among the examples of insects were CRISPR technique is used include the following: Drosophila melanogaster ${ }^{186}$, D. suzukii ${ }^{152,187}$, Aedes aegypti ${ }^{188}$, Spodoptera littoralis ${ }^{189}$, Locusta migratoria ${ }^{190}$, Gryllus bimaculatus ${ }^{191}$ and Tribolium casteneum ${ }^{23}$ etc. however, despite several successes recorded there is still some noticeable drawbacks associated with the technique such as delivery technology ${ }^{192}$, off/on target effects ${ }^{193}$ etc. which can be overcome with meticulous planning and screening of gRNAs against off targets.

Using CRISPR-Cas9 genome editing systems, we successfully generated transgenic driver strains at the identified Tribolium castaneum Tc007675 potential early embryonic cellularization specific gene. The success recorded was in stark contrast to some noticeable failures in our lab for using CRISPR-Cas9 to edit the genome of Tribolium.

The driver strains can be used in a heterologous transactivator (tTA) binary system which could be adopted for sexing ${ }^{123,142,142,177}$, Female Specific Embryonic Lethality (FSEL) ${ }^{123}$ and early embryonic lethality ${ }^{134,138}$. I verified the driver line potential to drive tTA at cellularization stage by WMISH using tTA DIG-labelled antisense probe, were i observed transient expression 
of the tTA in the cytoplasm of the embryos though not as strong as the indigenous expression Tc007675 gene in wildtype embryos.

Also, using Recombinase Mediated Cassette Exchange (RMCE) ${ }^{67-69}$ we intended to create mutants for Tc007675 gene to verify the function of the gene. RMCE is a strategy where the gene of interest for knocking in/knocking out in a genome is flanked by inverted pairs of attB ${ }^{68}$ sites. The gene of interest is now interchanged with a pre-inserted cassette in the genome i.e. docking site. The gene is flanked by corresponding attP recognition sites ${ }^{194}$ in the pre-inserted cassette in the presence of recombinase. However, using the RMCE strategy we failed in creating a mutant for the gene for now.

The possible reason for the inability to generate a mutant could be for plethora of issues but the more obvious one could be because we used the hsp70 basal promoter endogenous to Drosophila to drive the phic31-nls-integrase. To overcome this problem, we probably must use the Tribolium endogenous hsp68 basal promoter.

\subsection{Novel genetic engineering approach for the management of coleopteran insect pests using CRISPR-Cas9 system for reproductive sterility.}

Sterilization ${ }^{111,112}$ is one of the most important component and critical aspect of the Sterile Insect Technique (SIT), because without effective and efficient sterilization procedure, the technique cannot be utilized effectively. Largely, sterilization has been achieved by ionization radiation ${ }^{151}$ with noticeable successes recorded. However, despite the wonderful efficiency that the system is offering in the management of human and agricultural insect pest as an alternative to the injurious use of current control measures such as chemical control measures, such that when the chromosome of a treated male organism is broken it prevent the development of resistance. It however, has some drawbacks as well, and this includes effects of the procedure are not only on sperm cells but on somatic cells ${ }^{112,118,195}$ which thus affect the competitiveness ${ }^{111,177}$ of the treated individuals, non-applicability of the procedure to other insect species, and non $100 \%$ sterilization achieved on some insect species have also been reported. Other drawbacks include effects of the radiation on the health of the persons/scientists working with it, in an event of leakage from the facility. 
To circumvent this problem, several scientists have come up with various laudable techniques such as the RIDL based system ${ }^{106}$, or a transgenic conditional embryonic lethality system 123,131. However, to also achieve reproductive sterility in a novel way, we proposed the used of CRISPR/Cas9 to drive Cas9 during spermatogenesis. The aim is to cause precise and efficient induced multiple Double Strand Breaks (DSBs) ${ }^{43,43,196}$ in the chromosome by targeting the repetitive sequences of transposable elements ${ }^{30,33}$. With the new proposed system, the males should be able to produce sperm normally, but the sperms will not be viable to fertilize the embryos due to embryonic aneuploidy which will lead to no embryonic development. The requirements needed to develop the proposed reproductive sterility system are: E/P of spermatogenesis specific gene to mediate tTA expression in the testis as shown above, Cas9 under the control of TRE and gRNAs.

To actualized our aim using Tribolium castaneum ${ }^{16}$ being the model insect for coleopteran insects, we utilized P/E of the Tribolium spermatogenesis specific gene Tc-B2t, which we cloned to mediate the expression of tTA. We therefore generated the F5_F1 strain that expressed the tTA spermatogenesis specifically. Having successfully generated the driver strain that express tTA in testis, we then generated a potential Cas9 effector strain and crossed it with driver strains. No observable expression pattern in the testis was observed to validate Cas9 molecules in the testis for now. Moreover, for the system to be complete we need to also generate gRNA expressing lines or a cassette expressing gRNAs for multiple repetitive genomic sites and to validate it via $\mathrm{qPCR}$. When all the various components are combined the reproductive sterility will be achieved.

Thus, inducing reproductive sterility using a CRISPR/Cas9-based approach will ultimately solve the drawbacks such as male fitness of the generated males because the system has no effect on the somatic cells, also the production of sperm will be continuous with chromosomal breaks. Also, no adverse effect on human/environmental health is expected unlike in the use of radiation. Also, the system can be combined with sexing (FSEL) and sperm making system in an AW-SIT to manage coleopteran insect pests such as the Red Palm Weevil (RPW). 


\section{Outlook}

1) Make more crosses of the generated and verified $1.4 \mathrm{~Kb}$ and $2.1 \mathrm{~Kb} \mathrm{P} / \mathrm{E}$ driver line with the reporter and sexing effector lines to acertain the viability of the line to drive with the right controls included (Chapter 2)

2) Generate more Cas9 expressing effector lines that will be used for the sperm chromosome experiment and test the efficiency of the B2t-tTA (F5_F2) line (Chapter $3)$.

3) Search for genes such as the orthologs of Dm-Bam to provide guides for the CRISPRCas9 sperm chromosomal shredding experiment (Chapter 3).

4) Exchanging the $D m h s p 70$ basal promoter with $T c h s p 68$ basal promoter and repeat the RMCE experiment to generate Tc007675 mutant for further downstream experiment (Chapter 4). 


\section{References:}

1. Tsakas, S. \& Marmaras, V. J. Insect immunity and its signalling: an overview. Invertebrate Survival Journal 7, 228-238 (2010).

2. Chapman, R. F., Simpson, S. J. \& Douglas, A. E. The Insects: Structure and Function. (Cambridge University Press, 2013).

3. Encyclopedia of Insects - 2nd Edition. https://www.elsevier.com/books/encyclopedia-ofinsects/resh/978-0-12-374144-8.

4. Wojciechowska, M., Stepnowski, P. \& Gołębiowski, M. The use of insecticides to control insect pests. Invertebrate Survival Journal 13, 210-220 (2016).

5. Zhang, S.-Q. et al. Evolutionary history of Coleoptera revealed by extensive sampling of genes and species. Nature Communications 9, 1-11 (2018).

6. Slipinski, S. A., Leschen, R. a. B. \& Lawrence, J. F. Order Coleoptera Linnaeus, 1758. In : Zhang, Z.-Q. (Ed.) Animal biodiversity: An outline of higher-level classification and survey of taxonomic richness. Zootaxa 3148, 203-208 (2011).

7. Grove, S. J. \& Stork, N. E. An inordinate fondness for beetles. Invert. Systematics 14, 733-739 (2000).

8. R.A. Crowson, 1981. The biology of the Coleoptera. https://www.zin.ru/Animalia/Coleoptera/rus/crows981.htm.

9. Pai, A. Tribolium ş. in Encyclopedia of Animal Behavior (Second Edition) (ed. Choe, J. C.) 231241 (Academic Press, 2019). doi:10.1016/B978-0-12-809633-8.01216-4.

10. Brown, S. J. et al. The Red Flour Beetle, Tribolium castaneum (Coleoptera): A Model for Studies of Development and Pest Biology. Cold Spring Harbor Protocols 2009, pdb.emo126pdb.emo126 (2009).

11. Kumar, H. et al. Red flour beetle (Tribolium castaneum): From population genetics to functional genomics. Vet World 11, 1043-1046 (2018). 
12. Rust-Red Flour Beetle (Tribolium Castaneum): Distribution and Life Cycle. Your Article Library https://www.yourarticlelibrary.com/zoology/rust-red-flour-beetle-tribolium-castaneumdistribution-and-life-cycle/23997 (2014).

13. Schultheis, D. et al. A Large Scale Systemic RNAi Screen in the Red Flour Beetle Tribolium castaneum Identifies Novel Genes Involved in Insect Muscle Development. G3 (Bethesda) 9, 1009-1026 (2019).

14. Hunt, T. et al. A Comprehensive Phylogeny of Beetles Reveals the Evolutionary Origins of a Superradiation. Science 318, 1913-1916 (2007).

15. Holditch, Z. \& Smith, A. D. Priority determines Tribolium competitive outcome in a food-limited environment. PLOS ONE 15, e0235289 (2020).

16. Richards, S. et al. The genome of the model beetle and pest Tribolium castaneum. Nature $\mathbf{4 5 2}$, 949-955 (2008).

17. Chapman, R. N. NUTRITIONAL STUDIES ON THE CONFUSED FLOUR BEETLE, TRIBOLIUM CONFUSUM DUVAL. J. Gen. Physiol. 6, 565-585 (1924).

18. Demuth, J. P. \& Wade, M. J. Population Differentiation in the Beetle Tribolium Castaneum. I. Genetic Architecture. Evolution 61, 494-509 (2007).

19. Joop, G. \& Vilcinskas, A. Coevolution of parasitic fungi and insect hosts. Zoology 119, 350-358 (2016).

20. Rylee, J. C., Siniard, D. J., Doucette, K., Zentner, G. E. \& Zelhof, A. C. Expanding the genetic toolkit of Tribolium castaneum. PLOS ONE 13, e0195977 (2018).

21. Pavlopoulos, A., Berghammer, A. J., Averof, M. \& Klingler, M. Efficient transformation of the beetle Tribolium castaneum using the Minos transposable element: quantitative and qualitative analysis of genomic integration events. Genetics 167, 737-746 (2004).

22. Trauner, J. et al. Large-scale insertional mutagenesis of a coleopteran stored grain pest, the red flour beetle Tribolium castaneum, identifies embryonic lethal mutations and enhancer traps. BMC Biol 7, 73 (2009). 
23. Gilles, A. F., Schinko, J. B. \& Averof, M. Efficient CRISPR-mediated gene targeting and transgene replacement in the beetle Tribolium castaneum. Development 142, 2832-2839 (2015).

24. Rubin, G. M. \& Spradling, A. C. Genetic transformation of Drosophila with transposable element vectors. Science 218, 348-353 (1982).

25. Handler, A. M., Gomez, S. P. \& O’Brochta, D. A. A functional analysis of the P-element genetransfer vector in insects. Archives of Insect Biochemistry and Physiology 22, 373-384 (1993).

26. Beall, E. L. \& Rio, D. C. Drosophila P-element transposase is a novel site-specific endonuclease. Genes Dev 11, 2137-2151 (1997).

27. Rio, D. C. \& Rubin, G. M. Identification and purification of a Drosophila protein that binds to the terminal 31-base-pair inverted repeats of the P transposable element. Proc Natl Acad Sci U S A 85, 8929-8933 (1988).

28. Handler, A. M. Use of the piggyBac transposon for germ-line transformation of insects. Insect Biochem. Mol. Biol. 32, 1211-1220 (2002).

29. Atkinson, P. W., Pinkerton, A. C. \& O’Brochta, D. A. Genetic Transformation Systems in Insects. Annual Review of Entomology 46, 317-346 (2001).

30. Warren, W. D., Atkinson, P. W. \& O’Brochta, D. A. The Hermes transposable element from the house fly, Musca domestica, is a short inverted repeat-type element of the hobo, Ac, and Tam3 (hAT) element family. Genetics Research 64, 87-97 (1994).

31. Subramanian, R., Cathcart, L., Krafsur, E., Atkinson, P. \& O’Brochta, D. Hermes Transposon Distribution and Structure in Musca domestica. The Journal of heredity 100, 473-80 (2009).

32. Medhora, M., Maruyama, K. \& Hartl, D. L. Molecular and functional analysis of the mariner mutator element Mos1 in Drosophila. Genetics 128, 311-318 (1991).

33. Franz, G. \& Savakis, C. Minos, a new transposable element from Drosophila hydei, is a member of the Tc1-like family of transposons. Nucleic Acids Res 19, 6646 (1991). 
34. Cary, L. C. et al. Transposon mutagenesis of baculoviruses: Analysis of Trichoplusia ni transposon IFP2 insertions within the FP-locus of nuclear polyhedrosis viruses. Virology 172, 156-169 (1989).

35. Horn, C., Schmid, B. G. M., Pogoda, F. S. \& Wimmer, E. A. Fluorescent transformation markers for insect transgenesis. Insect Biochem. Mol. Biol. 32, 1221-1235 (2002).

36. Pavlopoulos, A., Oehler, S., Kapetanaki, M. G. \& Savakis, C. The DNA transposon Minos as a tool for transgenesis and functional genomic analysis in vertebrates and invertebrates. Genome Biol 8, S2 (2007).

37. Handler, A. M., James, A. A. \& James, A. A. Insect Transgenesis : Methods and Applications. (CRC Press, 2000). doi:10.1201/9781420039399.

38. Lynch, J. A. et al. The phylogenetic origin of oskar coincided with the origin of maternally provisioned germ plasm and pole cells at the base of the Holometabola. PLoS Genet 7, e1002029 (2011).

39. Schmitt-Engel, C. et al. The iBeetle large-scale RNAi screen reveals gene functions for insect development and physiology. Nat Commun 6, 1-10 (2015).

40. Bucher, G., Scholten, J. \& Klingler, M. Parental RNAi in Tribolium (Coleoptera). Curr. Biol. 12, R85-86 (2002).

41. Brodersen, P. \& Voinnet, O. The diversity of RNA silencing pathways in plants. Trends Genet. 22, 268-280 (2006).

42. Vilcinskas, A. Insect Biotechnology. (Springer Science \& Business Media, 2010).

43. Fire, A. et al. Potent and specific genetic interference by double-stranded RNA in Caenorhabditis elegans. Nature 391, 806-811 (1998).

44. Hannon, G. J. RNA interference. Nature 418, 244-251 (2002).

45. Hammond, S. M., Boettcher, S., Caudy, A. A., Kobayashi, R. \& Hannon, G. J. Argonaute2, a link between genetic and biochemical analyses of RNAi. Science 293, 1146-1150 (2001). 
46. Mlotshwa, S. et al. DICER-LIKE2 plays a primary role in transitive silencing of transgenes in Arabidopsis. PLoS One 3, e1755 (2008).

47. Zamore, P. D., Tuschl, T., Sharp, P. A. \& Bartel, D. P. RNAi: double-stranded RNA directs the ATP-dependent cleavage of mRNA at 21 to 23 nucleotide intervals. Cell 101, 25-33 (2000).

48. Buchon, N. \& Vaury, C. RNAi: a defensive RNA-silencing against viruses and transposable elements. Heredity 96, 195-202 (2006).

49. Pooggin, M. M. RNAi-mediated resistance to viruses: a critical assessment of methodologies. Curr Opin Virol 26, 28-35 (2017).

50. Berkhout, B. \& Haasnoot, J. The interplay between virus infection and the cellular RNA interference machinery. FEBS Letters 580, 2896-2902 (2006).

51. Kusaba, M. RNA interference in crop plants. Current Opinion in Biotechnology 15, 139-143 (2004).

52. Wang, M.-B. \& Metzlaff, M. RNA silencing and antiviral defense in plants. Current Opinion in Plant Biology 8, 216-222 (2005).

53. Zhai, Z., Sooksa-nguan, T. \& Vatamaniuk, O. K. Establishing RNA Interference as a ReverseGenetic Approach for Gene Functional Analysis in Protoplasts. Plant Physiology 149, 642-652 (2009).

54. Lipardi, C., Wei, Q. \& Paterson, B. M. RNA Silencing in Drosophila. Acta Histochemica Et Cytochemica 36, 123-134 (2003).

55. Hoffmann, T., Kalinowski, G. \& Schwab, W. RNAi-induced silencing of gene expression in strawberry fruit (Fragaria $x$ ananassa) by agroinfiltration: a rapid assay for gene function analysis. Plant J. 48, 818-826 (2006).

56. Gilchrist, E. \& Haughn, G. Reverse genetics techniques: engineering loss and gain of gene function in plants. Brief Funct Genomics 9, 103-110 (2010).

57. Ran, F. A. et al. Genome engineering using the CRISPR-Cas9 system. Nat Protoc 8, 2281-2308 (2013). 
58. Karginov, F. V. \& Hannon, G. J. The CRISPR system: small RNA-guided defense in bacteria and archaea. Mol Cell 37, 7 (2010).

59. Xu, Y. \& Li, Z. CRISPR-Cas systems: Overview, innovations and applications in human disease research and gene therapy. Computational and Structural Biotechnology Journal 18, 2401$2415(2020)$.

60. Rath, D., Amlinger, L., Rath, A. \& Lundgren, M. The CRISPR-Cas immune system: Biology, mechanisms and applications. Biochimie 117, 119-128 (2015).

61. Campenhout, C. V. et al. Guidelines for optimized gene knockout using CRISPR/Cas9. BioTechniques 66, 295-302 (2019).

62. Barrangou, R. et al. CRISPR Provides Acquired Resistance Against Viruses in Prokaryotes. Science 315, 1709-1712 (2007).

63. Brouns, S. J. J. et al. Small CRISPR RNAs Guide Antiviral Defense in Prokaryotes. Science 321, 960-964 (2008).

64. Marraffini, L. A. \& Sontheimer, E. J. CRISPR Interference Limits Horizontal Gene Transfer in Staphylococci by Targeting DNA. Science 322, 1843-1845 (2008).

65. Jinek, M. et al. A Programmable Dual-RNA-Guided DNA Endonuclease in Adaptive Bacterial Immunity. Science 337, 816-821 (2012).

66. Wimmer, E. A. Insect transgenesis by site-specific recombination. Nature Methods 2, 580-582 (2005).

67. Sauer, B. \& Henderson, N. Site-specific DNA recombination in mammalian cells by the Cre recombinase of bacteriophage P1. Proc. NatI. Acad. Sci. U.S.A. 85, 5166-5170 (1988).

68. O'Gorman, S., Fox, D. T. \& Wahl, G. M. Recombinase-mediated gene activation and site-specific integration in mammalian cells. Science 251, 1351-1355 (1991).

69. Thorpe, H. M. \& Smith, M. C. In vitro site-specific integration of bacteriophage DNA catalyzed by a recombinase of the resolvase/invertase family. Proc. Natl. Acad. Sci. U.S.A. 95, 5505-5510 (1998). 
70. Abi-Ghanem, J. et al. Engineering of a target site-specific recombinase by a combined evolution- and structure-guided approach. Nucleic Acids Res 41, 2394-2403 (2013).

71. Haghighat-Khah, R. E. et al. Site-specific cassette exchange systems in the Aedes aegypti mosquito and the Plutella xylostella moth. PLOS ONE 10, e0121097 (2015).

72. Nimmo, D. D., Alphey, L., Meredith, J. M. \& Eggleston, P. High efficiency site-specific genetic engineering of the mosquito genome. Insect Mol Biol 15, 129-136 (2006).

73. Belteki, G., Gertsenstein, M., Ow, D. W. \& Nagy, A. Site-specific cassette exchange and germline transmission with mouse ES cells expressing фC31 integrase. Nat Biotechnol 21, 321324 (2003).

74. Bethke, J., Redak, R. \& Paine, T. Screens deny specific pests entry to greenhouses. Calif. Agric. 48, 37-40 (1994).

75. Trenbath, B. R. Intercropping for the management of pests and diseases. Field Crops Research 34, 381-405 (1993).

76. Francis, C. A. CROP ROTATIONS. in Encyclopedia of Soils in the Environment (ed. Hillel, D.) 318322 (Elsevier, 2005). doi:10.1016/B0-12-348530-4/00253-8.

77. Rämert, B., Lennartsson, M. \& Davies, G. The use of mixed species cropping to manage pests and diseases - theory and practice. (2002).

78. Ghorbani, R. 18 - Reducing copper-based fungicide use in organic crop production systems. in Handbook of Organic Food Safety and Quality (eds. Cooper, J., Niggli, U. \& Leifert, C.) 392-412 (Woodhead Publishing, 2007). doi:10.1533/9781845693411.3.392.

79. Perdikis, D., Fantinou, A. \& Lykouressis, D. Enhancing pest control in annual crops by conservation of predatory Heteroptera. Biological Control 59, 13-21 (2011).

80. Sueldo, M. R., Bruzzone, O. A. \& Virla, E. G. Characterization of the Earwig, Doru lineare, as a Predator of Larvae of the Fall Armyworm, Spodoptera frugiperda: A Functional Response Study. Journal of Insect Science 10, 1-10 (2010). 
81. Llácer, E., Altube, M. \& Jacas, J. Evaluation of the efficacy of Steinernema carpocapsae in a chitosan formulation against the red palm weevil, Rhynchophorus ferrugineus, in Phoenix canariensis. BioControl 54, 559-565 (2008).

82. Dembilio, O., Quesada-Moraga, E., Santiago-Alvarez, C. \& Jacas, J. A. Potential of an indigenous strain of the entomopathogenic fungus Beauveria bassiana as a biological control agent against the Red Palm Weevil, Rhynchophorus ferrugineus. J Invertebr Pathol 104, 214-221 (2010).

83. Gozel, U., Gozel, C., Yurt, Ç. \& Inci, D. Efficacy of Entomopathogenic Nematodes on The Red Palm Weevil Rhynchophorus ferrugineus (Olivier, 1790) (Coleoptera: Curculionidae) Larvae. 4, 4436-4439 (2015).

84. Sewify, G., Belal, M. \& A, A.-A. Use of the Entomopathogenic Fungus, Beauveria bassiana for the biological control of the Red Palm Weevil, Rhynchophorus ferrugineus Olivier. Egyptian Journal of Biological Pest Control 19, 157-163 (2009).

85. Gindin, G., Levski, S., Glazer, I. \& Soroker, V. Evaluation of the entomopathogenic fungiMetarhizium anisopliae andBeauveria bassiana against the red palm weevilRhynchophorus ferrugineus. Phytoparasitica 34, 370-379 (2006).

86. Manachini, B., Schillaci, D. \& Arizza, V. Biological Responses of Rhynchophorus ferrugineus (Coleoptera: Curculionidae) to Steinernema carpocapsae (Nematoda: Steinernematidae). J Econ Entomol 106, 1582-1589 (2013).

87. Alfazairy, A. Antimicrobial activity of certain essential oils against hindgut symbionts of the drywood termite Kalotermes flavicollis Fabr. and prevalent fungi on termite-infested wood. Journal of Applied Entomology 128, 554-560 (2004).

88. JACOBSON, M. Natural Insect Attractants and Repellents, New Tools in Pest Control. in Natural Pest Control Agents vol. 53 17-26 (AMERICAN CHEMICAL SOCIETY, 1966).

89. Isman, M. Insect antifeedants. Pesticide Outlook 13, 152-157 (2002). 
90. Koul, O. Chapter 17 - Antifeedant Phytochemicals in Insect Management (so Close yet so Far). in Ecofriendly Pest Management for Food Security (ed. Omkar) 525-544 (Academic Press, 2016). doi:10.1016/B978-0-12-803265-7.00017-8.

91. Tewari, S., Leskey, T. C., Nielsen, A. L., Piñero, J. C. \& Rodriguez-Saona, C. R. Chapter 9 - Use of Pheromones in Insect Pest Management, with Special Attention to Weevil Pheromones. in Integrated Pest Management (ed. Abrol, D. P.) 141-168 (Academic Press, 2014). doi:10.1016/B978-0-12-398529-3.00010-5.

92. Chowanski, S., Kudlewska, M., Marciniak, P. \& Rosinski, G. Synthetic Insecticides - is There an Alternative? Polish Journal of Environmental Studies 23, 291-302 (2014).

93. Duke, S. O. et al. Natural Toxins for Use in Pest Management. Toxins (Basel) 2, 1943-1962 (2010).

94. WHO | Handbook for integrated vector management. WHO http://www.who.int/neglected_diseases/vector_ecology/resources/9789241502801/en/.

95. Ehler, L. E. Integrated pest management (IPM): definition, historical development and implementation, and the other IPM. Pest Management Science 62, 787-789 (2006).

96. Sharma, H. C. Biotechnological Approaches for Pest Management and Ecological Sustainability. (CRC Press, 2008). doi:10.1201/9781420088489.

97. Klassen, W. \& Curtis, C. F. History of the Sterile Insect Technique. in Sterile Insect Technique: Principles and Practice in Area-Wide Integrated Pest Management (eds. Dyck, V. A., Hendrichs, J. \& Robinson, A. S.) 3-36 (Springer Netherlands, 2005). doi:10.1007/1-4020-4051-2_1.

98. Knipling, E. F. Possibilities of Insect Control or Eradication Through the Use of Sexually Sterile Males. J Econ Entomol 48, 459-462 (1955).

99. Helinski, M. E., Parker, A. G. \& Knols, B. G. Radiation-induced sterility for pupal and adult stages of the malaria mosquito Anopheles arabiensis. Malaria Journal 5, 41 (2006).

100. Dyck, V. A., Hendrichs, J. \& Robinson, A. S. Sterile Insect Technique: Principles and Practice in Area-Wide Integrated Pest Management. (Springer Science \& Business Media, 2006). 
101. Hendrichs, J., Franz, G. \& Rendon, P. Increased effectiveness and applicability of the sterile insect technique through male-only releases for control of Mediterranean fruit flies during fruiting seasons. Journal of Applied Entomology 119, 371-377 (1995).

102. Hendrichs, M., Wornoayporn, V., Katsoyannos, B. \& Hendrichs, J. Quality Control Method to Measure Predator Evasion in Wild And Mass-Reared Mediterranean Fruit Flies (Diptera: Tephritidae). Florida Entomologist 90, 64-70 (2009).

103. Wimmer, E. A. Eco-friendly insect management. Nat Biotechnol 23, 432-433 (2005).

104. Bushland, R. C. \& Hopkins, D. E. Sterilization of Screw-Worm Flies with X-Rays and GammaRays. J Econ Entomol 46, 648-656 (1953).

105. Franz, G., Gencheva, E. \& Kerremans, Ph. Improved stability of genetic sex-separation strains for the Mediterranean fruit fly, Ceratitis capitata. Genome 37, 72-82 (1994).

106. Alphey, L. et al. Genetic control of Aedes mosquitoes. Pathog Glob Health 107, 170-179 (2013).

107. Morrison, N. et al. Genetic improvements to the Sterile Insect Technique for Agricultural Pests. AsPac J Mol Biol Biotechnol 18, (2010).

108. Enkerlin, W. R. Impact of Fruit Fly Control Programmes Using the Sterile Insect Technique. in Sterile Insect Technique: Principles and Practice in Area-Wide Integrated Pest Management (eds. Dyck, V. A., Hendrichs, J. \& Robinson, A. S.) 651-676 (Springer Netherlands, 2005). doi:10.1007/1-4020-4051-2_25.

109. El-Hindi, M. W., Asaker, B. A. A. \& Kichaoui, A. Y. E. Isolation, Molecular Identification and under Lab Evaluation of the Entomopathogenic Fungi M. anisopliae and B. bassiana against the Red Palm Weevil R. ferrugineus in Gaza Strip. Advances in Microbiology 7, 720-726 (2017).

110. Sterile insect technique. https://www.iaea.org/topics/sterile-insect-technique (2016).

111. Zhao, J. et al. Sperm precedence pattern and the effect of irradiation on male mating competition in the oriental fruit fly, Bactrocera dorsalis. 5. 
112. Ernawan, B., Tambunan, U. S. F., Sugoro, I. \& Sasmita, H. I. Effects of gamma irradiation doserate on sterile male Aedesaegypti. in 020010 (2017). doi:10.1063/1.4985401.

113. Robinson, A. S. Mutations and their use in insect control. Mutat Res 511, 113-132 (2002).

114. Rendón, P., Mclnnis, D., Lance, D. \& Stewart, J. Medfly (Diptera: Tephritidae) genetic sexing: large-scale field comparison of males-only and bisexual sterile fly releases in Guatemala. J. Econ. Entomol. 97, 1547-1553 (2004).

115. Tan, A. et al. Transgene-based, female-specific lethality system for genetic sexing of the silkworm, Bombyx mori. PNAS 110, 6766-6770 (2013).

116. Heinrich, J. C. \& Scott, M. J. A Repressible Female-Specific Lethal Genetic System for Making Transgenic Insect Strains Suitable for a Sterile-Release Program. Proceedings of the National Academy of Sciences of the United States of America 97, 8229-8232 (2000).

117. Yan, Y. \& Scott, M. J. A transgenic embryonic sexing system for the Australian sheep blow fly Lucilia cuprina. Scientific Reports 5, 16090 (2015).

118. Guerfali, M. M. et al. Fitness and Reproductive Potential of Irradiated Mass-Reared Mediterranean Fruit Fly Males Ceratitis capitata (Diptera: Tephritidae): Lowering Radiation Doses. flen 94, 1042-1050 (2011).

119. Pagabeleguem, S. et al. A Molecular Method to Discriminate between Mass-Reared Sterile and Wild Tsetse Flies during Eradication Programmes That Have a Sterile Insect Technique Component. PLoS Negl Trop Dis 10, (2016).

120. Dolezal, P., Okrouhlik, J. \& Davidkova, M. Fine fluorescent powder marking study of dispersal in the spruce bark beetle, Ips typographus (Coleoptera: Scolytidae). Eur. J. Entomol. 113, (2016).

121. Wimmer, E. A. Eco-friendly insect management. Nat Biotechnol 23, 432-433 (2005).

122. Vreysen, M. J. B., Robinson, A. S. \& Hendrichs, J. Area-Wide Control of Insect Pests: From Research to Field Implementation. (Springer Science \& Business Media, 2007). 
123. Ogaugwu, C. E., Schetelig, M. F. \& Wimmer, E. A. Transgenic sexing system for Ceratitis capitata (Diptera: Tephritidae) based on female-specific embryonic lethality. Insect Biochemistry and Molecular Biology 43, 1-8 (2013).

124. Schetelig, M. \& Handler, A. A transgenic embryonic sexing system for Anastrepha suspensa (Diptera: Tephritidae). Insect biochemistry and molecular biology 42, 790-5 (2012).

125. Eckermann, K. N. et al. Perspective on the combined use of an independent transgenic sexing and a multifactorial reproductive sterility system to avoid resistance development against transgenic Sterile Insect Technique approaches. BMC Genet 15 Suppl 2, S17 (2014).

126. Scolari, F. et al. Fluorescent sperm marking to improve the fight against the pest insect Ceratitis capitata (Wiedemann; Diptera: Tephritidae). New Biotechnology 25, 76-84 (2008).

127. Schetelig, M. F., Wimmer, E. A., Scolari, F., Gasperi, G. \& Handler, E. A. New genetic tools for improving SIT in Ceratitis capitata: embryonic lethality and sperm marking.

128. Meza, J. S. et al. Comparison of classical and transgenic genetic sexing strains of Mediterranean fruit fly (Diptera: Tephritidae) for application of the sterile insect technique. PLoS One 13, (2018).

129. Aketarawong, N., Isasawin, S., Laohakieat, K. \& Thanaphum, S. Genetic stability, genetic variation, and fitness performance of the genetic sexing Salaya1 strain for Bactrocera dorsalis, under long-term mass rearing conditions. BMC Genetics 21, 131 (2020).

130. Thomas, D. D., Donnelly, C. A., Wood, R. J. \& Alphey, L. S. Insect Population Control Using a Dominant, Repressible, Lethal Genetic System. Science 287, 2474-2476 (2000).

131. Schetelig, M. F. \& Handler, A. M. Strategy for enhanced transgenic strain development for embryonic conditional lethality in Anastrepha suspensa. PNAS 109, 9348-9353 (2012).

132. Alphey, L. et al. Genetic technologies to enhance the Sterile Insect Technique (SIT). 8.

133. Bakri, A., Mehta, K. \& Lance, D. R. Sterilizing Insects with lonizing Radiation. in Sterile Insect Technique (eds. Dyck, V. A., Hendrichs, J. \& Robinson, A. S.) 233-268 (Springer-Verlag, 2005). doi:10.1007/1-4020-4051-2_9. 
134. Schetelig, M. F., Caceres, C., Zacharopoulou, A., Franz, G. \& Wimmer, E. A. Conditional embryonic lethality to improve the sterile insect technique in Ceratitis capitata(Diptera: Tephritidae). BMC Biol 7, 4 (2009).

135. Gossen, M. \& Bujard, H. Tight control of gene expression in mammalian cells by tetracyclineresponsive promoters. Proc. Natl. Acad. Sci. U.S.A. 89, 5547-5551 (1992).

136. Urlinger, S. et al. Exploring the sequence space for tetracycline-dependent transcriptional activators: novel mutations yield expanded range and sensitivity. Proc. Natl. Acad. Sci. U.S.A. 97, 7963-7968 (2000).

137. Bello, B., Resendez-Perez, D. \& Gehring, W. J. Spatial and temporal targeting of gene expression in Drosophila by means of a tetracycline-dependent transactivator system. 10.

138. Horn, C. \& Wimmer, E. A. A transgene-based, embryo-specific lethality system for insect pest management. Nature Biotechnology 21, 64-70 (2003).

139. Dyck, V. A. Rearing codling moth for the sterile insect technique. (Food and Agriculture Organization, 2010).

140. Hagler, J. R. \& Jackson, C. G. Methods for marking insects: current techniques and future prospects. Annu. Rev. Entomol. 46, 511-543 (2001).

141. Berghammer, A. J., Klingler, M. \& A. Wimmer, E. A universal marker for transgenic insects. Nature 402, 370-371 (1999).

142. Catteruccia, F., Benton, J. P. \& Crisanti, A. An Anopheles transgenic sexing strain for vector control. Nature Biotechnology 23, 1414-1417 (2005).

143. MI, A., Da, O., Pw, A. \& Cs, L. Stable, Germ-Line Transformation of Culex Quinquefasciatus (Diptera: Culicidae). Journal of medical entomology https://pubmed.ncbi.nlm.nih.gov/11580043/ (2001) doi:10.1603/0022-2585-38.5.701.

144. Smith, R. C., Walter, M. F., Hice, R. H., O’Brochta, D. A. \& Atkinson, P. W. Testis-specific expression of the beta2 tubulin promoter of Aedes aegypti and its application as a genetic sexseparation marker. Insect Mol. Biol. 16, 61-71 (2007). 
145. Am, H. Understanding and improving transgene stability and expression in insects for SIT and conditional lethal release programs. Insect Biochem Mol Biol 34, 121-130 (2004).

146. Handler, A. M., Zimowska, G. J. \& Horn, C. Post-integration stabilization of a transposon vector by terminal sequence deletion in Drosophila melanogaster. Nature Biotechnology 22, 11501154 (2004).

147. Horn, C. \& Handler, A. M. Site-specific genomic targeting in Drosophila. Proc. Natl. Acad. Sci. U.S.A. 102, 12483-12488 (2005).

148. Dafa'alla, T. H. et al. Transposon-free insertions for insect genetic engineering. Nature Biotechnology 24, 820-821 (2006).

149. Venken, K. J. T. et al. Versatile P[acman] BAC libraries for transgenesis studies in Drosophila melanogaster. Nature Methods 6, 431-434 (2009).

150. Nene, V. et al. Genome Sequence of Aedes aegypti, a Major Arbovirus Vector. Science 316, 1718-1723 (2007).

151. Al-Ayedh, H. Y. \& Rasool, K. G. Determination of the optimum sterilizing radiation dose for control of the red date palm weevil Rhynchophorus ferrugineus Oliv. (Coleoptera: Curculionidae). Crop Protection 29, 1377-1380 (2010).

152. Ahmed, H. M. M., Hildebrand, L. \& Wimmer, E. A. Improvement and use of CRISPR/Cas9 to engineer a sperm-marking strain for the invasive fruit pest Drosophila suzukii. BMC Biotechnol 19, 85 (2019).

153. Michiels, F., Gasch, A., Kaltschmidt, B. \& Renkawitz-Pohl, R. A 14 bp promoter element directs the testis specificity of the Drosophila beta 2 tubulin gene. EMBO J 8, 1559-1565 (1989).

154. Zimowska, G. J., Nirmala, X. \& Handler, A. M. The beta2-tubulin gene from three tephritid fruit fly species and use of its promoter for sperm marking. Insect Biochem. Mol. Biol. 39, 508-515 (2009).

155. Bevis, B. J. \& Glick, B. S. Rapidly maturing variants of the Discosoma red fluorescent protein (DsRed). Nat. Biotechnol. 20, 83-87 (2002). 
156. Cinelli, R. A. et al. The enhanced green fluorescent protein as a tool for the analysis of protein dynamics and localization: local fluorescence study at the single-molecule level. Photochem. Photobiol. 71, 771-776 (2000).

157. Krzywinska, E. \& Krzywinski, J. Analysis of expression in the Anopheles gambiae developing testes reveals rapidly evolving lineage-specific genes in mosquitoes. BMC Genomics 10, 300 (2009).

158. Scolari, F. et al. Polyandry in the medfly - shifts in paternity mediated by sperm stratification and mixing. BMC Genet 15, S10 (2014).

159. Bertin, S. et al. Sperm storage and use in polyandrous females of the globally invasive fruitfly, Ceratitis capitata. Journal of Insect Physiology 56, 1542-1551 (2010).

160. Droge-Young, E. M., Belote, J. M., Perez, G. S. \& Pitnick, S. Resolving mechanisms of short-term competitive fertilization success in the red flour beetle. Journal of Insect Physiology 93-94, 110 (2016).

161. Culliney, T. Crop Losses to Arthropods. in Integrated Pest Management vol. 3 201-225 (2014).

162. Vincent, C., Weintraub, P. G., Hallman, G. J. \& Fleurat-Lessard, F. Insect management with physical methods in pre- and post-harvest situations. in Integrated Pest Management (eds. Radcliffe, E. B., Hutchison, W. D. \& Cancelado, R. E.) 309-323 (Cambridge University Press, 2008). doi:10.1017/СВ09780511626463.025.

163. Mantzoukas, S. et al. Postharvest Treatment of Tribolium confusum Jacquelin du Val Adults with Commercial Biopesticides. Agriculture 9, 226 (2019).

164. Singh, S. \& Prakash, S. Development of resistance in Tribolium castaneum, Herbst (Coleoptera: Tenebrionidae) towards deltamethrin in laboratory. 3, 4 (2013).

165. Bernardes, M. F. F., Pazin, M. \& Dorta, L. C. P. and D. J. Impact of Pesticides on Environmental and Human Health. Toxicology Studies - Cells, Drugs and Environment (2015) doi:10.5772/59710. 
166. Zhang, C. X., Lee, M. P., Chen, A. D., Brown, S. D. \& Hsieh, T. Isolation and characterization of a Drosophila gene essential for early embryonic development and formation of cortical cleavage furrows. J Cell Biol 134, 923-934 (1996).

167. Sonawane, A. R. et al. Understanding Tissue-Specific Gene Regulation. Cell Rep 21, 1077-1088 (2017).

168. Schweisguth, F., Lepesant, J. A. \& Vincent, A. The serendipity alpha gene encodes a membraneassociated protein required for the cellularization of the Drosophila embryo. Genes \& Development 4, 922-931 (1990).

169. FlyBase Homepage. https://flybase.org/.

170. iBeetle. http://ibeetle-base.uni-goettingen.de/.

171. Herndon, N. et al. Enhanced genome assembly and a new official gene set for Tribolium castaneum. BMC Genomics 21, 47 (2020).

172. Distler, J., Hoffmann, S., Büning, J. \& Rübsam, R. Functional Analysis of Spermatogenesis in Tribolium castaneum. (2008).

173. Laptev, I. A., Raevskaya, N. M., Filimonova, N. A. \& Sineoky, S. P. The piggyBac Transposon as a Tool in Genetic Engineering. Appl Biochem Microbiol 53, 874-881 (2017).

174. Handler, A. M. \& Ii, R. A. H. Germline transformation of Drosophila melanogaster with the piggyBac transposon vector. Insect Molecular Biology 8, 449-457 (1999).

175. Das, A. T., Tenenbaum, L. \& Berkhout, B. Tet-On Systems For Doxycycline-inducible Gene Expression. Curr Gene Ther 16, 156-167 (2016).

176. Yamamoto, D. S. et al. A synthetic male-specific sterilization system using the mammalian proapoptotic factor in a malaria vector mosquito. Sci Rep 9, (2019).

177. Meza, J. S. et al. Comparison of classical and transgenic genetic sexing strains of Mediterranean fruit fly (Diptera: Tephritidae) for application of the sterile insect technique. PLoS One 13, (2018). 
178. Urquidi, J., Brar, R. K., Rodriguez, S. \& Hansen, I. The development of new radiation protocols for insect sterilization using long wavelength x-rays. in 020010 (2015). doi:10.1063/1.4927187.

179. Ansai, S. \& Kinoshita, M. Targeted mutagenesis using CRISPR/Cas system in medaka. Biol Open 3, 362-371 (2014).

180. Yeo, N. C. et al. An enhanced CRISPR repressor for targeted mammalian gene regulation. Nat Methods 15, 611-616 (2018).

181. Xu, X. et al. Gene activation by a CRISPR-assisted trans enhancer. Elife 8, (2019).

182. Lei, Y., Huang, Y.-H. \& Goodell, M. A. DNA methylation and de-methylation using hybrid sitetargeting proteins. Genome Biology 19, 187 (2018).

183. Chen, L., Wang, G., Zhu, Y.-N., Xiang, H. \& Wang, W. Advances and perspectives in the application of CRISPR/Cas9 in insects. 9.

184. Sun, D., Guo, Z., Liu, Y. \& Zhang, Y. Progress and Prospects of CRISPR/Cas Systems in Insects and Other Arthropods. Front. Physiol. 8, (2017).

185. Gantz, V. M. Gene editing technologies and applications for insects. Current Opinion in Insect Science 7 (2018).

186. Urban, J. A. et al. The essential Drosophila CLAMP protein differentially regulates non-coding roX RNAs in male and females. Chromosome Res 25, 101-113 (2017).

187. Li, F. \& Scott, M. J. CRISPR/Cas9-mediated mutagenesis of the white and Sex lethal loci in the invasive pest, Drosophila suzukii. Biochemical and Biophysical Research Communications 469, 911-916 (2016).

188. Dong, S. et al. Heritable CRISPR/Cas9-Mediated Genome Editing in the Yellow Fever Mosquito, Aedes aegypti. PLOS ONE 10, e0122353 (2015).

189. Koutroumpa, F. A. et al. Heritable genome editing with CRISPR/Cas9 induces anosmia in a crop pest moth. Scientific Reports 6, 29620 (2016). 
190. Li, Y. et al. CRISPR/Cas9 in locusts: Successful establishment of an olfactory deficiency line by targeting the mutagenesis of an odorant receptor co-receptor (Orco). Insect Biochemistry and Molecular Biology 79, 27-35 (2016).

191. Awata, H. et al. Knockout crickets for the study of learning and memory: Dopamine receptor Dop1 mediates aversive but not appetitive reinforcement in crickets. Scientific Reports $\mathbf{5}$, 15885 (2015).

192. Chaverra-Rodriguez, D. et al. Targeted delivery of CRISPR-Cas9 ribonucleoprotein into arthropod ovaries for heritable germline gene editing. Nat Commun 9, 3008 (2018).

193. Naito, Y., Hino, K., Bono, H. \& Ui-Tei, K. CRISPRdirect: software for designing CRISPR/Cas guide RNA with reduced off-target sites. Bioinformatics 31, 1120-1123 (2015).

194. Baer, A. \& Bode, J. Coping with kinetic and thermodynamic barriers: RMCE, an efficient strategy for the targeted integration of transgenes. Curr Opin Biotechnol 12, 473-480 (2001).

195. Khan, S. A., Eggleston, H., Myles, K. M. \& Adelman, Z. N. Differentially and Co-expressed Genes in Embryo, Germ-Line and Somatic Tissues of Tribolium castaneum. G3 (Bethesda) 9, 23632373 (2019).

196. Brunner, E. et al. CRISPR-induced double-strand breaks trigger recombination between homologous chromosome arms. Life Science Alliance 2, (2019). 


\section{CURRICULUM VITAE}

Master of Philosophy Entomology

ISAH, MUSA DAN'AZUMI

Department of Developmental Biology,

Johann Friedrich Blumenbach,

Institute of Zoology and Anthropology,

Geörg-August-Universität Göttingen,

GZMB Ernst-Caspari Haus,

Justus-Von Liebig-Weg 11,

37077 Göttingen,

Germany.

\section{EDUCATION:}

01/10/2014- Present

Dr. rer. Nat. Geörg-August-Universität Göttingen, Germany.

Genetic engineering, Molecular Biology, Genome editing, Parental RNAi

Aug 2006-June 2008:

Master of Philosophy in Entomology, African Regional Post Graduate Programme In Insect Science (ARPPIS) Laboratory, University of Ghana, Legon, Greater Accra Region, Ghana.

Thesis: Isah, M. D. (2008). Damage by Prostephanus truncatus (Hon) (Coleoptera: Bostrichidae) to dry chips of yam, cocoyam, cassava and plantain. Mphil in Entomology Thesis, University of Ghana Legon: 109pp

Oct 1999-June 2004: $\quad$ BSc. Agricultural Science Honours Second Class Upper Division, University of Maiduguri, Borno State, Nigeria

Dissertation: Isah, M. D. (2004). Distribution Pattern of Some selected Field and Stored Products Insect Pest. Bachelor of Agriculture final year project, University of Maiduguri, Borno State, Nigeria, 75pp.

Jan 1996-Dec 1998: $\quad$ National Diploma in Agricultural Technology, Upper Credit, Abubakar Tatari Ali polytechnic, school of Agriculture, Bauchi State, Nigeria.

Dissertation: Isah, M. D. (1998). The effect of different levels of Agrolyser on the yield of maize, National Diploma final year project, school of Agriculture, Abubakar Tatari Ail polytechnic, Bauchi: 40pp. 


\section{SEMINARS/ CONFERENCES ATTENDED AND PRESENTATION:}

16-21 JUNE 2019: Development of transgenic sperm marking in Tribolium castaneum to enhanced effective monitoring of coleopteran insect pests in sterile insect technique program. Gordon Research Conference Mount Holyoke College in South Hadley MA United States (Poster Presentation).

11-14 Sept. 2017: Göttingen Biotech Symposium, German Primate Center DPZ, Göttingen, Germany, 26 September 2017 'Horizons in Molecular Biology Symposium. Max Plank Institute for biophysical Chemistry, Göttingen, Germany,

22-26 MAY 2017: Development of a Biotechnologically enhanced Sterile Insect Technique to Fight Coleopteran Insect Pests. Third FAO-IAEA International Conference on Area-wide Management of Insect Pests: Integrating the Sterile Insect and Related Nuclear and Other Techniques IAEA Headquarters, Vienna, Austria (Poster Presentation).

\section{ADDITIONAL SKILLS:}

Computer skills: $\quad$ Microsoft office, Photo editor, Genstat statistical software, SPSS 16.0, Stat view and other statistical software, adobe illustrator. Adobe photoshop.

Languages: $\quad$ English (Native speaker)

Hausa (Native speaker)

German (Basic)

Awards:

2016 - 2018: Evangelishes Studientenwerk e.V. Villigst, Stupendium for $\mathrm{PhD}$ student.

Dec 2005: Best Graduating Student Department of Crop Protection University of Maiduguri, Borno State Nigeria.

Feb 2003: Federal Government of Nigeria Scholarship (In Country: 3.00 CGPA Student). 
Aug 2006-2008: Awarded German Academic Exchange services (DAAD) Scholarship for In-Region Programme (DAADICIPE/ARPPIS) to pursue Postgraduate Studies (MPhil) at the University of Ghana, Legon.

\section{COURSES:}

- Entrepreneurship for Doctoral Candidates and Post Docs: organized by GGNB in Göttingen, 4 March 2016.

- GGNB course Next Generation Sequencing (NGS)-Analysis of RNAseq and CHIPseq data (A157/340208). 14 -16 November, 2017

- Adobe Illustrator Beginners' Course: Course number S44_2. 21 August, 2018.

- Chromatin-immunoprecipitation and epigenomic gene-profiling in the adult brain. 27 - 29 August 2018.

\section{PUBLICATION:}

- Kolja N. Eckermanna, Hassan M.M. Ahmeda, Mohammad KaramiNejadRanjbara, Stefan Dippela, Christian E. Ogaugwua, Peter Kitzmann, Musa D. Isah, Ernst A. Wimmer (2018). Hyperactive piggyBac transposase improves transformation efficiency in diverse insect species. $\begin{array}{lllll}\text { Insect Biochemistry and Molecular Biology } 98 & 16-24\end{array}$ https://doi.org/10.1016/j.ibmb.2018.04.001

- Donald A. Ukeh, Mandu J. Sambo and Musa D. Isah (2013). Response of Potential Stored Grain Insect Pests to BFL 225 Multi-Attractant Lure in Commercial Warehouses. Journal of Biology, Agriculture and Healthcare 3 (8): $87-91$

- Sylvia B.A. Umoetok, Musa D. Isah, Joseph A. Ukeh and Idorenyin A. Udo (2013). Evaluation of the Insecticidal and Deterrence Properties of Pepper Fruit (Dennetia tripetala (G. Baker)) and Ginger (Zingiber officinale Roscoe) against Maize Weevil Sitophilus zeamais (Motsch.). Journal of Biology, Agriculture and Healthcare 3 (6): 76 - 82 
- Collins N. Ehisianya, Donald A. Ukeh, Musa D. Isah, Ndowa E. S. Lale and Odidika C. Umeozor (2013). Field Efficacy of Neem Seed Oil and Diazinon in the Management of Sweetpotato Weevil, Cylas puncticollis (Boh.) in South-eastern Nigeria. Journal of Plant Studies. 2 (1): 135-144

- Isah, M. D., Abdullahi, G. and Sastawa, B. M. (2012). Distribution Patterns of Some Field and Stored Commodities in Maiduguri, North Eastern Nigeria: Implication for their management. International Journal of Applied Research and Technology. 1(6): 227 - 232.

- Isah, M. D., Sylvester, A. and Zakka, U. (2012). Potentials of Plantain and Cocoyam dried chips as growth medium for Prostephanus truncatus (Horn) (Coleoptera: Bostrichidae). International Journal of Applied Research and Technology. 1(5): 133 - 140.

- M.D. Isah, J.N. Ayertey, D.A. Ukeh and S.B.A. Umoetok (2012). Damage and Weight Loss to Dried Chips of Cassava, Cocoyam, Yam and Plantain Exposed to Prostephanus truncatus (Horn) (Coleoptera: Bostrichidae) over Three Different Time Durations. Journal of Entomology 9(3): 137-145.

- Abdullahi G., Sule H., Chimoya I. A. and Isah, M.D. (2011). Diversity and Relative Distribution of Honeybees Foraging Plants in some selected Reserves in Mubi Region, Sudan Savannah Ecological Zone of Nigeria. Advances in Applied Research, 2(5): 388-395. Available online at Pelagia Research Library

- B.M. Sastawa, M.D. Isah, G. Abdullahi and H. Sule (2011). Panicle Insect Pests of Millet in Maiduguri, Nigerian Sudan Savanna: Farmers' Perception, Relative Abundance and Associated Grain Yield Loss. Journal of Arid Agriculture, 20(2011): 130-139

- B.M. Sastawa, M.D. Isah, G. Abdullahi and H. Sule (2010). Effects of Intersowing Multiple Legumes on Millet Damage and Grain Loss by Pachnoda interrupta (Olivier) at Maiduguri, Nigeria. Proceedings of the $6^{\text {th }}$ National Conference on Organic Agriculture held at Faculty of Agriculture, University of Maiduguri, Maiduguri, $21^{\text {st }}-24^{\text {th }}$ November $2010.152-$ $158 \mathrm{pp}$.

- Isah, M.D., Ayertey, J.N and Boateng, B.A. (2009). Suitability of Dried Chips of Plantain, Cocoyam, Yam and Cassava for the Development of the 
Larger Grain Borer Prostephanus truncatus (Horn) (Coleoptera: Bostrichidae). International Journal of Pure and Applied Sciences, 3(4): 12 -20 . 